\title{
Passive wake detection using seal whisker-inspired sensing
}

\author{
by \\ Heather Rachel Beem \\ B.S., Oklahoma State University (2008) \\ http://orcid.org/0000-0003-2893-5177
}

Submitted to the Department of Mechanical Engineering and the Joint Program in Applied Ocean Science and Engineering in partial fulfillment of the requirements for the degree of

Doctor of Philosophy

at the

\section{MASSACHUSETTS INSTITUTE OF TECHNOLOGY}

and the

\section{WOODS HOLE OCEANOGRAPHIC INSTITUTION}

February 2015

\section{(C)2015 Heather Rachel Beem. All rights reserved.}

The author hereby grants to MIT and to WHOI permission to reproduce and distribute publicly paper and electronic copies of this thesis document in whole or in part in any medium now known or hereafter created.

Author

Joint Program in Applied Ocean Science and Engineering August 29, 2014

Certified by

Michael S. Triantafyllou

Thesis Supervisor, Professor, MIT

Accepted by

David Hardt

Graduate Officer, Mechanical Engineering, MIT

Accepted by

Henrik Schmidt

Chairman, Joint Committee for Applied Ocean Science and Engineering,

MIT/WHOI 


\title{
Passive wake detection using seal whisker-inspired sensing
}

by

\author{
Heather Rachel Beem
}

\begin{abstract}
Submitted to the Department of Mechanical Engineering and the Joint Program in Applied Ocean Science and Engineering on August 29, 2014, in partial fulfillment of the requirements for the degree of Doctor of Philosophy
\end{abstract}

\begin{abstract}
This thesis is motivated by a series of biological experiments that display the harbor seal's extraordinary ability to track the wake of an object several seconds after it has swum by. They do so despite having auditory and visual cues blocked, pointing to use of their whiskers as sensors of minute water movements. In this work, I elucidate the basic fluid mechanisms that seals may employ to accomplish this detection. Key are the unique flow-induced vibration properties resulting from the geometry of the harbor seal whisker, which is undulatory and elliptical in cross-section.

First, the vortex-induced vibration (VIV) characteristics of the whisker geometry are tested. Direct force measurements and flow visualizations on a rigid whisker model undergoing a range of 1-D imposed oscillations show that the geometry passively reduces VIV (factor of $>10$ ), despite contributions from effective added mass and damping. Next, a biomimetic whisker sensor is designed and fabricated. The rigid whisker model is mounted on a four-armed flexure, allowing it to freely vibrate in both in-line and crossflow directions. Strain gauges on the flexure measure deflections at the base. Finally, this device is tested in a simplified version of the fish wake - seal whisker interaction scenario. The whisker is towed behind an upstream cylinder with larger diameter. Whereas in open water the whisker exhibits very low vibration when its long axis is aligned with the incoming flow, once it enters the wake it oscillates with large amplitude and its frequency coincides with the Strouhal frequency of the upstream cylinder. This makes the detection of an upstream wake as well as an estimation of the size of the wake-generating body possible. A slaloming motion among the wake vortices causes the whisker to oscillate in this manner. The same mechanism has been previously observed in energy-extracting foils and trout actively swimming behind bluff cylinders in a stream.
\end{abstract}

Thesis Supervisor: Michael S. Triantafyllou

Title: Professor of Mechanical and Ocean Engineering 


\section{Acknowledgments}

This thesis represents a small piece of my work and life over the last six years. Thanks to all of the people along the way who have made the full story.

Many thanks to my advisor, Professor Triantafyllou, who has opened my eyes to the fascinating world of biomimetics. Through his example, I have learned to appreciate the power and beauty of using fundamental science to build systems and understanding from the ground up.

My committee members have provided support, practical advice, and great examples of mentorship. Thank you volunteering your time and input.

To all of the people who've been in the Towing Tank Lab group during my time here, thanks for being a wonderful group of research companions. Ajay, Alan, Amy, Audren, Audrey, Dilip, Dixia, Fangfang, Gabriel, Haining, Hendrik, Jacob, James, Jeff, Martin, Mohsen, Stephanie S., Vignesh: You have been there in the nitty-gritty troubleshooting and envisioning the big picture. From you, I have learned both how to be a better experimentalist and the joy in mulling over abstract concepts in fluid mechanics. I count myself blessed to have been in this group together with you.

Dave, Jason, Gabe, Pablo, Remi, and Yahya were postdocs when we first met. Thank you for being approachable and sharing much needed advice all along the way. Your love of science has inspired me to be more inquisitive.

Brandon, Chris, Matthew, and Patrick were undergrads or high school students who spent their summers working in the Tow Tank with me. Thanks for giving me the chance to practice being a mentor, moving this research forward a lot faster than I could do on my own, and helping me lift the mega-whisker in and out of the tank! May you all keep on building and exploring.

Thanks to Kathy Streeter and the New England Aquarium staff for helping me understand more about real harbor seals. They graciously collected shed whisker specimens and allowed me to take many close-up pictures of their seals.

Thanks to all the people who helped me prepare for and make it through research

cruises: Eric Hayden, Terry Hammar, and Hanu Singh for contributing their expertise 
to help me make my first ocean-ready sensor, all parties involved in the first UNOLS Chief Scientist Training Cruise series, and my JP friends (Abby, Amalia, Dan O., Derya, Elise, Holly, Jeff K., Kalina, LiLing, Stephanie P., Wu-Jung), with whom I've also endured many long commutes.

There are many folks who helped me learn to communicate this work in a way that is understandable to the rest of the world. Thanks to Lonny Lippsett (who actually read this entire document!) and Ken Kostel for their guidance on writing, Felice Frankel for help with figures, and John Freidah for producing a wonderful video.

Thanks to my PEN/D-Lab colleagues: Anna, Aron, Brianna, Fareeha, Grace, Jessica, Maddie, Ned, and Pedro. Through our work, I've not only kept myself busy outside of lab, but experienced the fun of joining up with brilliant minds and scheming up new ideas. Thanks for all of the adventures here and abroad.

When I first came to graduate school, I had two expectations: 1- that I would be challenged academically and 2- that I would be in a exciting city. While those two things indeed came true, along the way I found that life in grad school was richer than I had imagined. The call God placed on my heart to follow him has led to life, hope, and joy. Thank you to my MIT family: the members of GCF and Tang Small Group. It is through your lives that I have been challenged and inspired to hear the call.

To my roommates (and basically-roommates) in 20B: I've lived longer with some of you than I have anyone besides my own flesh and blood! But God has used this challenge of staying planted to cause me to dig deep roots. Thank you for being fertile soil in which I could grow, and praise God that the fruit has naturally been born. Ange, Ellann, Michelle, Nan, Stephanie, Wei Shan, it's been an amazing to grow up together with you, dream about the future, and run this marathon side by side. All the tough times and the joyful times we've shared together- I wouldn't trade them for anything.

Thank you to my boyfriend Dan, my best friend and faithful companion. The redemption, love, and joy that have come from our time together are written between 
each line here.

Mom, Dad, Jenn, and Robert: thank you for your unconditional love. Thank you for inspiring me to live fully, humbly, and righteously. May God bless you deeply.

Finally, thanks to the many financial sponsors of this project, including the NDSEG Fellowship, MIT Presidential Fellowship, Link Foundation Fellowship, and the National Research Foundation Singapore through the Singapore MIT Alliance for Research and Technology's Center for Environmental Sensing and Modeling interdisciplinary research program. Support was also provided by the Office of Naval Research through grant N00014-13-1-0059, monitored by Dr. Thomas Swean, Jr., the MIT Sea Grant Program, and the William I. Koch Chair in Marine Technology. 


\section{Contents}

$\begin{array}{lll}1 & \text { Introduction } & 31\end{array}$

$1.0 .1 \quad$ How might seals detect a fish trail? . . . . . . . . . . . . 32

$1.1 \quad$ Flow Sensing $\ldots \ldots \ldots \ldots \ldots$

$1.1 .1 \quad$ Pinniped Sensory Systems $\ldots \ldots \ldots$. . . . . . . . . . 34

1.1 .2 Vibrissae (Whiskers) . . . . . . . . . . . . . . . . 37

1.1 .3 Other Animals $\ldots \ldots \ldots \ldots$. . . . . . . . . . 47

$1.1 .4 \quad$ Hydrodynamic Stimuli . . . . . . . . . . . . . . . . . . 49

1.1.5 Hydrodynamic Perception Experiments with Pinnipeds . . . . 50

1.1.6 What is the mechanism of detection? . . . . . . . . . 56

$1.2 \quad$ Vortex-Induced Vibrations (VIV) $\ldots \ldots \ldots \ldots \ldots$

1.2 .1 Why does VIV occur? . . . . . . . . . . . . . . . . 57

1.2 .2 Model . . . . . . . . . . . . . . . . . . . . . . . . 59

$1.2 .3 \quad$ VIV Suppression Mechanisms $\ldots \ldots \ldots$. . . . . . . 65

1.3 Synthesis of Sensing and Vibrations $\ldots \ldots \ldots \ldots$

1.3 .1 Previous Work . . . . . . . . . . . . . . . . . . . . 69

$1.3 .2 \quad$ Hypothesis $\ldots \ldots \ldots \ldots \ldots$

$1.4 \quad$ Outline of Current Study . . . . . . . . . . . . . . . . . . . 71

\begin{tabular}{|lll}
2 & Whisker in Open Flow: Forced Vibrations & 73
\end{tabular}

2.1 Motivation . . . . . . . . . . . . . . . . . . . . . 73

$2.1 .1 \quad$ Whisker in Frontal Flows $\left(\alpha=0^{\circ}\right) \quad \ldots \ldots \ldots \ldots \ldots$

2.1 .2 Whisker at Angles of Attack $\left(\alpha>0^{\circ}\right) \ldots \ldots \ldots \ldots$

2.2 Methodologies . . . . . . . . . . . . . . . 76 
2.2 .1 Force Measurements: Forced Vibrations . . . . . . . . 76

$2.2 .2 \quad$ Particle Image Velocimetry (PIV): Steady Flow . . . . . . . . 81

$2.2 .3 \quad$ Particle Image Velocimetry (PIV): Forced Vibrations . . . . . 82

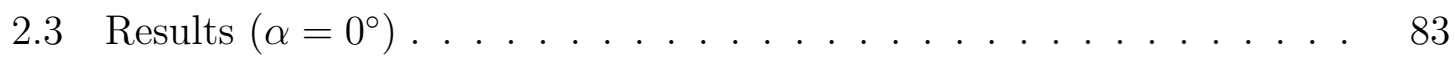

$2.3 .1 \quad$ Force Measurements . . . . . . . . . . . . . . . . . . . . . . 83

$2.3 .2 \quad$ PIV $\ldots \ldots \ldots \ldots \ldots \ldots$

$2.4 \quad \operatorname{Results}\left(\alpha>0^{\circ}\right) \ldots \ldots \ldots \ldots$

$2.4 .1 \quad$ Force Measurements . . . . . . . . . . . . . . . . . . . . . . 89

2.4 .2 PIV $\ldots \ldots \ldots \ldots \ldots \ldots$

2.5 Discussion $\ldots \ldots \ldots \ldots$

2.5 .1 Whisker in Frontal Flows. . . . . . . . . . . . . . . . . . 99

2.5 .2 Whisker at Angles of Attack . . . . . . . . . . . . . 100

2.5 .3 Note on the geometry . . . . . . . . . . . . . . . . . . 101

\begin{tabular}{lll}
\hline 3 & Flow Sensor Design & 103
\end{tabular}

3.1 Introduction . . . . . . . . . . . . . . . . . . . . . . . 103

3.1 .1 Previous Work . . . . . . . . . . . . . . . . . . . 104

3.2 Flow Sensor - Version 1 . . . . . . . . . . . . . . . . . . . 105

$3.2 .1 \quad$ Design . . . . . . . . . . . . . . . . . . . . . . . . . 105

3.2 .2 Calibration . . . . . . . . . . . . . . . 108

3.2 .3 Towing Tests $\ldots \ldots \ldots \ldots \ldots$

$3.2 .4 \quad$ Research Cruise Deployment . . . . . . . . . . . . . . . . . . 114

3.3 Flow Sensor: Version $2 \ldots \ldots$

$3.3 .1 \quad$ Design . . . . . . . . . . . . . . . . . . . . . . . . . . . . . . 118

3.3 .2 Calibration . . . . . . . . . . . . . . . 120

$3.3 .3 \quad$ Coupling . . . . . . . . . . . . . . . . . . . . . . . . . . . . . . 121

3.4 Conclusion . . . . . . . . . . . . . . . . . . . . . . . . 123

3.4 .1 Version $1 \ldots \ldots \ldots \ldots$

3.4 .2 Version $2 \ldots \ldots \ldots \ldots \ldots$

$3.4 .3 \quad$ Future Work . . . . . . . . . . . . . . . . . . . . . . 125 
4 Whisker in Vortex Wake: Free Vibrations 127

4.1 Motivation . . . . . . . . . . . . . . . . . . . . . . . . . 127

$4.2 \quad$ Methodologies . . . . . . . . . . . . . . . . . . . . . . . . . 128

$4.2 .1 \quad$ Note on the geometry . . . . . . . . . . . . . . . . . . . 129

4.2 .2 Strain Measurements: Free Vibrations _. . . . . . . . . . . 130

$4.2 .3 \quad$ Dye Visualizations: Free Vibrations . . . . . . . . . . . . . . . 132

4.2 .4 Wake Frequency Measurements . . . . . . . . . . . . . . 133

$4.2 .5 \quad$ Analysis . . . . . . . . . . . . . . . . . . 133

4.3 Results . . . . . . . . . . . . . . . . . . . . . . . . . . . . . . . . 134

$4.3 .1 \quad$ VIV response $\ldots \ldots \ldots \ldots \ldots \ldots$

$4.3 .2 \quad$ Whisker response within a wake $\left(d_{c y l}=2.5 d_{w}, d_{x}=8 d_{w}\right) \ldots 136$

4.3 .3 Dye visualizations in wake $\ldots \ldots \ldots$. . . . . . . . . . 138

$4.3 .4 \quad$ Cross-correlation analysis $\ldots \ldots \ldots \ldots \ldots$

4.3.5 Comparison between the whisker and the cylinder and elliptical geometries . . . . . . . . . . . . . . . . . . . 141

$4.3 .6 \quad$ Wake Detectability . . . . . . . . . . . . . . . . 143

4.4 Discussion . . . . . . . . . . . . . . . . . . . . . . . . . . . . . . . 144

$\begin{array}{lll}5 \text { Conclusions } & 147\end{array}$

5.1 Overview . . . . . . . . . . . . . . . . . . . . . . . . . . . 147

$5.2 \quad$ Principal Contributions $\ldots \ldots \ldots \ldots \ldots$

5.2 .1 Forced vibrations on a whisker model . . . . . . . . . . . . . 148

5.2 .2 Design of whisker sensor $\ldots \ldots \ldots \ldots$

5.2 .3 Mechanism of wake detection . . . . . . . . . . . . . 151

5.3 General Implications $\ldots \ldots \ldots \ldots$

$5.3 .1 \quad$ Sensory systems employed $\ldots \ldots \ldots$. . . . . . . . . . . . 152

5.3 .2 Comparison with other species . . . . . . . . . . . . . . . 152

5.4 Future Work . . . . . . . . . . . . . . . . . . . . . . . . . 153

$5.4 .1 \quad$ Optimizing the whisker geometry $\ldots \ldots \ldots \ldots \ldots$

5.4 .2 Determining the limits of detection $\ldots \ldots \ldots \ldots \ldots$ 
5.4 .3 Implementing a control loop . . . . . . . . . . . . . . . . 155

5.4 .4 Determining the function of the whisker array $\ldots \ldots \ldots$. . 156

\begin{tabular}{|l|l|}
\hline A Whisker Image Database & 159
\end{tabular}

B Omnidirectional Whisker-Inspired Geometries 165

B.1 Experiment Motivation . . . . . . . . . . . . . . . . . . . . . 165

B.2 Shape Design . . . . . . . . . . . . . . . . . . . . . 165

B.2.1 Direct Conversion to Circular cross sections . . . . . . . . 166

B.2.2 "Spiral-like" Shapes . . . . . . . . . . . . . . . . . . . . 166

B.2.3 Twisted Ellipse . . . . . . . . . . . . . . . . . . . 168

B.3 Forced Vibrations: Force Measurements. . . . . . . . . . . . . . . . . 169

B.3.1 Apparatus . . . . . . . . . . . . . . . . . . . . . . 169

B.4 Results . . . . . . . . . . . . . . . . . . . . . . 170

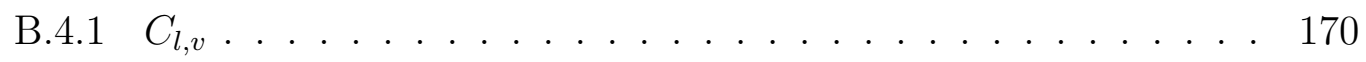

\begin{tabular}{ll}
\hline C Effect of Flexibility on VIV & 173
\end{tabular}

C.1 Motivation . . . . . . . . . . . . . . . . . . . . . . 173

C.2 Experiment Setup. . . . . . . . . . . . . . . . . . . . 173

C.2.1 Model Fabrication . . . . . . . . . . . . . . . . . . 173

C.2.2 Mounting Scheme . . . . . . . . . . . . . . . 175

C.2.3 Modeling . . . . . . . . . . . . . . . . . . . . . . . 175

C.2.4 Motion Tracking . . . . . . . . . . . . . . . . 177

C.3 Results . . . . . . . . . . . . . . . . . . . . . . . . . . 178

C.3.1 Accelerometer . . . . . . . . . . . . . . . . . . . 178

C.3.2 Motion Tracking of Model . . . . . . . . . . . . . . . . 179

C.4 Discussion and Future Work . . . . . . . . . . . . . . . . . . . 181 


\section{List of Figures}

1-1 A harbor seal wearing a blindfold and earmuffs, ready for an experiment. Photo from Marine Science Center, Rostock University. . . . . 32

1-2 Schematic of a seal searching for a fish by using its whiskers to detect the hydrodynamic trail. . . . . . . . . . . . . . . . . . . . . . . . 33

1-3 A harbor seal's eye covers a wide pupillary range to aid visual acuity in both water and air. The pupil goes from (a) circular in dark water to

(b) a vertical slit on land, as light increases. The scale bar corresponds to $1 \mathrm{~cm}$. Figure from [53]. . . . . . . . . . . . . . . . . 35

1-4 Sketches of (a) an echolocation experiment and (b) a magnetic sensing experiment conducted with harbor seals. Figure from [109]. . . . . . . 36

1-5 (Left) Thermogram of a harbor seal's face immediately after leaving the water. The warmest places are around the eyes, nostrils, and vibrissal pads. Figure from [21]. (Right top) Photograph and (Right bottom) thermogram of the vibrissal pad of a harbor seal Figure. The follicles have higher temperature than the rest of the pad. Figure from [90]. . 39

1-6 A naso-nasal greeting between a harbor seal mother and her pup, demonstrating the use of vibrissae to communicate socially. Figure from [110]. . . . . . . . . . . . . . . . . . . . . . . . . . . . . . . . . . . . . . . . . 39

1-7 (a) Different shapes that a sea lion was asked to distinguish using vibrissal tactile sensing. (b) Experimental setup for the blindfolded sea lion. Figures from [19]. . . . . . . . . . . . . . . . . . . . . . . . . 40 
1-8 (a) A diagram of the follicle-sinus-complex system, which holds each vibrissa and connects it to the brain. (b) A ringed seal's FSC partially split open. 1: upper cavernous sinus, 2: ring sinus, 3: lower cavernous sinus, C: capsule, Cm: collagenous membrane, R: liquid-filled cleft, Rw: ringwulst, $\mathrm{N}$ : nerve bundle penetrating the capsule, G: groove. Figures from [69]. . . . . . . . . . . . . . . . . . . . . . . . . . . 42

1-9 (a) The somatosensory cortex portion of the brain. The points on the body that stimulate the labeled regions on the cortex from (b) the trunk, (c) the head, (d) the vibrissae (marked in size according to length and diameter). The vibrissae are seen to take up a sizable portion of the total area. Figure from [79]. . . . . . . . . . . . . . . 42

1-10 Sketch of experiment, in which a harbor seal was asked to identify whether or not a rod in contact with its vibrissae was vibrating. Figure from [109].]. . . . . . . . . . . . . . . . . . . . . . . . . . . . . . . . . . . . . . 44

1-11 A few of the different vibrissal morphologies seen in pinnipeds. Phocid seals generally exhibit an undulatory profile unlike the smooth profile seen on otariids. Figure from [45]. . . . . . . . . . . . . . . . . . . . . 45

1-12 Pictures of a vibrissa from a ringed seal (top) and California sea lion (bottom). Figure from [45]. . . . . . . . . . . . . . . . . . . . . 45

1-13 The number of bumps is seen to increases somewhat linearly with vibrissa length. Figure from [46]. . . . . . . . . . . . . . . . . . 46

1-14 Examples of other animals with vibrissae: (a) manatee (figure from

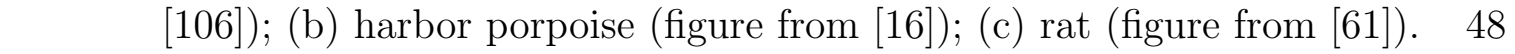

1-15 Examples of other animals with hairlike structures: (a) star-nosed mole and (b) tentacled snake (both figures from [14]). . . . . . . . . . . . . 48

1-16 Sketch of the reverse von Kármán vortex wake left by a swimming fish, which produces thrust. Figure from [29]. . . . . . . . . . . . . . . 49 
1-17 Experiment setup for dipole stimuli detection. A harbor seal wearing a blindfold and earmuffs was stationed at a set distance from an oscillating sphere. Vibrissal detection threshold of the resulting water movements was measured. Figure from [26]. . . . . . . . . . . . . . . 51

1-18 Schematic of the submarine trail detection experiment. A harbor seal wearing a blindfold is asked to locate a toy submarine, which swam ahead on a linear path in a random direction several seconds earlier. The yellow line marks the actual trail, the red line indicates a potential search path the seal could've taken, and the blue semicircle marks the locations where the sub could have landed. The probability of the seal finding the submarine by arbitrarily choosing a direction is $P \leq 0.04$.

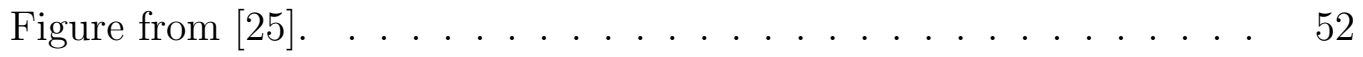

1-19 The two paths taken by the harbor seal (red arrow) to locate the toy submarine (yellow arrow). In the absence of acoustical cues, the seal followed the sharp turn that the sub also took (solid yellow line). When the sub engine was audible, the seal followed a direct path to it (dotted yellow line). Figure from [25]. . . . . . . . . . . . . . . . 53

1-20 (A) One seal swims ahead on the blue path. A second seal swims behind several seconds later and takes the red path, despite being blindfolded. (B) Same setup, but the second seal now employs a more distinctively undulatory pattern to the search. Figure from [118]. . . 54

1-21 Different shapes that a seal was trained to distinguish based solely on

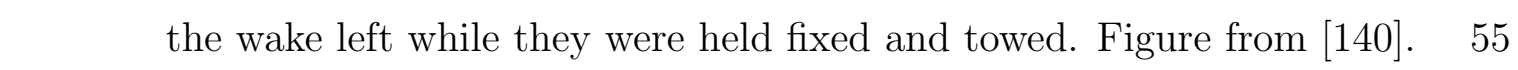

1-22 Ocean structures, such as offshore platforms (figure from [66]) and cables (figure from [35]), are subject to VIV. . . . . . . . . . . 57

1-23 A bluff body in a uniform flow can be simply represented as a 1-degreeof-freedom linear oscillator. The free stream approaches with velocity $U$ causing vortex shedding at a frequency of $f_{s} . \ldots . . . . . .59$

1-24 Examples of VIV suppression devices: (a) fairing, (b) splitter plate, (c) flag, and (d) a cylinder with helical strakes. Figures from [35]. . . 66 
1-25 The drag coefficient of various VIV suppression devices is higher than that of a smooth cylinder at certain Reynolds numbers. This is especially true for a cylinder with helical strakes. Figure from [35]. . . . . 67

1-26 (a) A cylinder with bumpy protrusions placed at $45^{\circ}$ angular separation and with pitch of 7 diameters. Figure from [100]. (b) The wake behind this model ranges from wide to narrow, depending on whether the model has a peak or trough in the disturbance there. Figure from [99]. 67

1-27 Maximum transverse vibration amplitude of different geometries. As the mass-damping $\left(m^{*} \zeta\right)$ decreases, all geometries vibrate with higher amplitude. solid circle: smooth circular cylinder; plus sign: sinuous cylinder; others: bumpy cylinders with various bump sizes). Figure from $[99]$.

1-28 Schematic of expected response of the whiskers as the seal is swimming: the whiskers undergo significant VIV, making for a "noisy" signal. . . 69

1-29 Schematic of the hypothesis behind this thesis. In open flow, the whiskers produce low VIV. This enables the seal to more easily detect unsteady flow features, which induce large response. . . . . . . . 70

2-1 Close-up view of the whiskers on a harbor seal's muzzle. The "beaded" pattern indicates an undulatory geometry. Photo taken courtesy of the New England Aquarium. . . . . . . . . . . . . . . . . . . . 74

2-2 (Top) Side view and (Bottom) front view of a fabricated replica of the whisker test section, capturing the asymmetric undulatory geometry. Stereolithography was used to create this plastic hollow structure. Dimensions were taken directly from [58] and enlarged by a factor of 50.75

2-3 Seals's whiskers may face different orientations. Photo taken at the New England Aquarium. . . . . . . . . . . . . . . . . . . . 76 
2-4 Schematic of the forced vibration setup. The model hangs vertically in the water tank. It is directly attached to a force sensor and a linear stage that drives a sinusoidal motion (an example trajectory is depicted in red) while the carriage moves the setup down the length of the tank

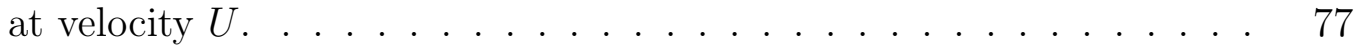

2-5 Orientation of a cross section of the model. The solid line cross section represents the $\alpha=0^{\circ}$ case and the dotted line cross section represents the $\alpha>0^{\circ}$ cases. The projected diameter $\left(d^{*}\right)$ is the dimension used for all cases and is the length perpendicular to the flow, which approaches

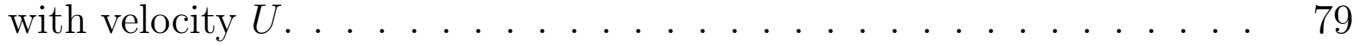

2-6 Schematic of the PIV setup. The model again hangs vertically in the water tank. A laser sheet illuminates a thin cross-section of the model and the fluid in that plane. A camera mounted below the tank captures the motion of neutrally buoyant particles in the water. . . . . . . . . 82

2-7 $C_{d}$ is plotted as a function of amplitude ratio and reduced frequency for the circular cylinder, elliptical cylinder, and whisker (from left to right). $R e=5,300$. Asterisks indicate where experiments were performed. The contour levels are in increments of 0.1 up to 1 , then in increments of 0.25 to 1.5 , and in increments of 0.5 beyond that. . . . . . . . . . . 83

2-8 $C_{L v}$ is plotted as a function of amplitude ratio and reduced frequency. The region of positive values indicates where VIV occurs. The size of this region decreases from the circular cylinder to the elliptical cylinder to the whisker (from left to right). The zero contour is depicted in bold. $R e=5,300$. Asterisks indicate where experiments were performed. The contour levels are in increments of 0.5 down to -0.5 , then in increments of 2 from -2 on, and in increments of -6 beyond that. . 84 
2-9 Variation seen in the whisker $C_{l, v}$ plot. The vertical bars indicate the probability that that point on the plot would show a positive $C_{l, v}$ value. The solid black dots indicate the average (over 10 trials) $C_{l, v}$ value at each cycle of oscillation. The scatter plots (red) show the spread of the 10 individual values at each cycle. The standard deviation decreases over time, indicating that lock-in is reached after some initial start-up phase. ......................... 86

2-10 $C_{m}$ contours plotted as a function of amplitude ratio and reduced frequency for the circular cylinder, elliptical cylinder, and whisker (from left to right). The zero $C_{L v}$ contour is copied over and shown in black. The contour levels are in increments of 1 and the 3.5 contour is included as well. Asterisks indicate where experiments were performed. . . . . 87

2-11 Vorticity contours of a 2-D slice of the wake behind the elliptical cylinder (left) and whisker (right) models undergoing steady tow. A standard von Kármán vortex street appears behind the ellipse, but the whisker decorrelates the vortex shedding, causing the vortex street to appear much farther downstream, where the forcing on the body is reduced. . . . . . . . . . . . . . . . . . . . . 88

2-12 Vorticity contours of a 2-D slice of the wake behind the elliptical cylinder (left) and whisker (right) models undergoing the forced vibration run corresponding to their maximum VIV amplitude. A standard von Kármán vortex street appears behind both models. . . . . . . . . . . 89

2-13 Drag coefficient during forced vibrations for (left column) ellipse and (right column) whisker at (a,b) 15; (c,d) 30; (e,f) 45; (g,h) 60; (i,j) $75 ;(\mathrm{k}, 1) 90^{\circ}$. Contour levels for 15 and $30^{\circ}$ are in increments of 0.1 . Otherwise they are in increments of 0.5. . . . . . . . . . . . . . . 92

2-14 $C_{l, v}$ contour for (left column) ellipse and (right column) whisker at (a,b) 15; (c,d) 30; (e,f) 45; (g,h) 60; (i,j) 75; (k,l) 90. Contour levels are in increments of 1. . . . . . . . . . . . . . . . . . . . . . 94 
2-15 The peak amplitude of vibration for the elliptical cylinder and whisker increases as the angle of attack increases. $R e=5,300-7,030$ from $\alpha=0^{\circ}-45^{\circ}$ and $R e=2,530-3,210$ from $\alpha=60^{\circ}-90^{\circ}$. . . . . . . 95

2-16 Effective added mass coefficient during forced vibrations for (left column) ellipse and (right column) whisker at (a,b) 15; (c,d) 30; (e,f) 45; (g,h) 60; (i,j) 75; (k,l) 90. Contour levels are in increments of 0.5. . . 97

2-17 PIV of the wake behind an elliptical cylinder (left) and whisker (right)

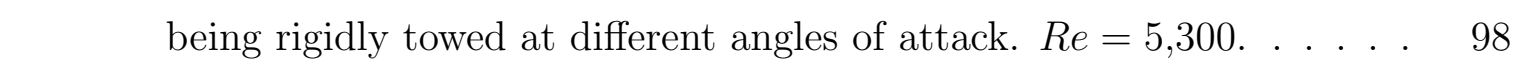

2-18 The wake shed behind the freely vibrating whisker for the (left) 45 and (right) $90^{\circ}$ cases. (Experiments conducted by B. Seyed-Aghazadeh and Y. Modarres-Sadeghi) . . . . . . . . . . . . . . . . . . . . . . . . . . 99

3-1 The whisker (yellow) mounted to a pressure housing, which contains four Bend Sensors (dark orange). . . . . . . . . . . . . . . 106

3-2 (a) A square plate (in black) at the end of a rod connected to the whisker pushes on the four Bend Sensors as the whisker deflects. (b) A silicone diaphragm (in orange) between the whisker and a retaining ring allows flexible motion. . . . . . . . . . . . . . . . . 106

3-3 The electronics that are stored inside a separate housing: circuit and data logger. The battery pack is not pictured. . . . . . . . . . . . . 107

3-4 Circuit diagram. The Bend Sensor signal lines are filtered and amplified.108

3-5 Setup for bench-top calibration. Deflection of the whisker was conducted in increments, using the ruler on the left as a reference. . . . . 108

3-6 Voltage output versus whisker tip displacement for each of the four Bend Sensors. . . . . . . . . . . . . . . . . . . . . . . 109 
3-7 Towing test setup. (Left) The sensor is mounted to the carriage, and a data logger records voltage output. Note: The wake generator (circular cylinder) pictured was not used for the experiments shown in this Chapter. (Right) Schematic of the inside of the sensor housing (top view). The Bend Sensors (purple) are numbered with this convention throughout. Note: For $\alpha=0^{\circ}$, Bend Sensors 1 and 2 are in the crossflow direction, but as $\alpha$ is increased to $90^{\circ}$, Bend Sensors 3 and 4 become fully in the crossflow direction. . . . . . . . . . . . . . . 111

3-8 Time traces of the Bend Sensor outputs. Black vertical lines indicate start and end of the carriage motion. (Top row) $\alpha=0^{\circ}$ : minimal change occurs. (Middle row) $\alpha=45^{\circ}$ : ramps and oscillations in voltage are seen. (Bottom row): $\alpha=90^{\circ}$ : larger oscillations occur in the crossflow (Sensors 3,4) and larger ramps occur inline (Sensors 1,2). Sensors 1 and 4 are shifted by a constant value to consolidate plots. . 112

3-10 Field test setup. The sensor is mounted to the CTD rosette in a manner that allows the whisker to encounter unobstructed flow. The view is from the port side of the ship. (Photo courtesy of Paul Suprenand) • 115

3-11 (a) Velocity measurements at 20m depth from the ADCP and (b) voltage output from the whisker-inspired sensor. . . . . . . . . . . . . . . 116

3-12 Orientation of the ship and sensor. The ship had a heading of $-120^{\circ}$ to $-140^{\circ}$ during the $10 \mathrm{~min}$ deployment. The arrow indicates the general flow direction shown by the ADCP. The numbers indicate the orientation of the corresponding Bend Sensors. . . . . . . . . . . . . . . . . 116

3-13 Isometric view of the wake sensor, which consists of a plastic whisker \begin{tabular}{l}
\hline model mounted on a four-armed flexing plate. Strain gauges on these \\
\begin{tabular}{|l}
\hline arms are mounted on these arms for measurement of the whisker's \\
\hline deflections. . . . . . . . . . . . . . . . . . . . . . . . . 119
\end{tabular}
\end{tabular} 
3-14 (a) A Solidworks simulation of the strain induced on the flexure as the whisker deflects. (b) Close-up of the flexure device. A spring steel plate made using the water jet is attached to a bushing (clear) and pin (gray) at the end of each arm. This design reduces coupling between the two axes by allowing rotation, rather than deflection in the off-axis. 119

3-15 The data-collection electronics. The strain gauges are conFigured in a Wheatstone bridge, using additional resistors. A PhidgetBridge is used to filter and transmit the voltages to a computer through USB. . 120

3-16 (Left) A linear fit was placed through data collected for different tip deflections of the whisker in $x$. (Right) A pluck test in air was used to find the natural frequency of the whisker in $y$. The values here $\left(V_{y n}, V_{x n}\right)$ are centered around zero by subtracting the mean of the signal.121

3-17 Picture of sensor assembly in the (left) normal configuration and (right) with the addition of set screws (circled in red) to restrict bushing rotation. A fourth set screw through the remaining bushing is not visible from this view. . . . . . . . . . . . . . . . . . . . . . . . . . . 122

3-18 Power density of signals from $y$-direction pluck tests in water for the (left) normal configuration and (right) configuration with no bushing rotation. . . . . . . . . . . . . . . . . . . 122

3-19 Vibrational amplitude in both axes when (left) no set screws were used and (right) set screws were used to restrict bushing rotation. $U_{r}=17$. 
4-1 (Left) Top view; and (Right) side view of the experimental setup. A hydrodynamic trail was generated in front of the whisker through the use of a cylinder, which shed a Karman vortex street. Three different downstream distances $\left(d_{x}\right)$ were tested for each of the three models, ranging from the near wake to the far wake. Three different cylinder sizes $\left(d_{c y l}\right)$ were also tested. Simultaneous measurement of the oncoming wake frequency and whisker response was done through use of a pressure sensor placed near the edge of the wake. A mariotte bottle supplied dye at a constant flow rate. A camera underneath the model captured a bottom-up view of the wake-whisker interaction. . . . . . 129

4-2 Two variations in the harbor seal whisker geometry are captured through the depicted parameters, which are shown here at the scale of the real seal. The inclination angles are $17.6^{\circ}$ and $15.27^{\circ}$ for the top and bottom cross-sections shown, respectively. The geometry from Hanke et al (2010) ("whisker \#1") has a $\lambda / D_{m} \approx 2$, whereas the new geometry ("whisker \#2") has a $\lambda / D_{m} \approx 5$. The models used in this chapter are scaled up from the dimensions shown here 30 and 20 times, respectively.130

4-3 The model whisker ("whisker \#1" pictured here) was mounted on a set of flexing plates that allow free vibration in in-line and crossflow directions. Bushings are used to reduce coupling between the axes. . . 131

4-4 VIV response of each model positioned in the streamlined direction $\left(\alpha=0^{\circ}\right)$. Bars depict data spread over three repeat runs. (Left) Both cross-flow and in-line amplitudes of the whisker and elliptical cylinder are significantly smaller than those of the circular cylinder. The frequencies at which the whisker and ellipse vibrate remain close to their natural frequency. The diagonal dotted line indicates a frequency that matches the Strouhal relation. (Right) Both whiskers and the elliptical cylinder vibrate with $A_{y}^{*}<0.2$. Whisker \#2 has the lowest VIV amplitude. . . . . . . . . . . . . . . . . . . . 135 
4-5 Dye visualizations of the wake behind a circular cylinder, elliptical cylinder, and whisker (from left to right) at $\alpha=0^{\circ}$. The first two objects shed regular 2S patterns, whereas the whisker \#1 model sheds an incoherent wake. Images were taken during runs corresponding to each model's peak amplitude: cylinder at $U^{*}=10$, ellipse at $U^{*}=28$, and whisker $\# 1$ at $U^{*}=28$. . . . . . . . . . . . . . . . . . . . . . 136

4-6 Dye visualization of the wake behind the whisker \#2 model. Similar to the whisker \#1 model, it sheds an incoherent wake. The image was taken during the run corresponding to the model's peak amplitude, which is at $U^{*}=32$. . . . . . . . . . . . . . . . . . . . 136

4-7 For a whisker within the wake of an upstream cylinder $\left(d_{c y l}=2.5 d_{w}\right)$ at a distance $d_{x}=8 d_{w}$ : (a) The whisker vibrates with far higher amplitude in the wake than it does on its own. (b) The peak frequency of the whisker's vibration synchronizes with the oncoming wake frequency. The diamonds and " $\mathrm{x}$ "s are the average over three repeat runs. The bars indicate the data spread of the measured wake frequency over the same three runs. . . . . . . . . . . . . . . . . . . . . . . . . 137

4-8 Schematic of the camera perspective of the dye runs. The bottom-up view shows vortices being shed by the wake generator on the right and impinging on a cross-section of the whisker on the left. . . . . . . . . 138

4-9 Sequence of dye visualizations (left) and schematics (right) showing the whisker's free vibration path through the vortex wake. The frequency of the whisker's motion aligns with the vortex wake frequency, as the whisker slaloms between the vortices. $\left(U^{*}=16, R e=3,180\right)$. . . . . 139

4-10 Sample result of the cross-correlation analysis. (Top) The normalized \begin{tabular}{l}
\hline signals of strain and pressure measured on the whisker and in the \\
\begin{tabular}{|c|}
\hline wake, respectively. (Bottom) The cross-correlation coefficient at each \\
\hline oscillation cycle. $\left(d_{x}=17 d_{w}, d_{c y l}=4 d_{w}, U^{*}=27\right)$. . . . . . . . . . . 140
\end{tabular}
\end{tabular} 
4-11 Cross-correlation metrics of the whisker vibration with the wake frequency for three different size cylinders at $d_{x}=15 d_{c y l}$. (Left) All cases see a high maximum cross-correlation coefficient. (Right) The percentage of the run where the whisker is "synchronized" generally increases with cylinder diameter. . . . . . . . . . . . . . . . . . . . . . . . 141

4-12 Average of percent synchronization across all speeds tested $\left(d_{c y l}=\right.$ $\left.44 d_{w}\right)$. The trends are displayed versus separation distance both in the downstream $\left(d_{x}\right)$ and lateral $\left(d_{y}\right)$ directions. Experiments with a lateral offset were conducted only on one side of the cylinder but the results are assumed to be symmetric and are therefore mirrored here. 142

4-13 The frequencies of response nondimensionalized by the theoretical frequency. At the close distance $\left(8 d_{w}\right)$, all four models vibrate at the theoretical wake frequency. As the distance increases to $17 d_{w}$, the elliptical cylinder and whisker continue to oscillate at the wake frequency, but the cylinder deviates from it. . . . . . . . . . . . . . . . . . 143

4-14 Vibration amplitudes of the circular cylinder, elliptical cylinder, and whisker (from left to right, respectively). The latter two vibrate more when they are in the wake of an upstream cylinder (colored lines) than they do in open water. The circular cylinder vibrates with a similar amplitude for all cases. The amplitudes of response decrease as the distance from the upstream cylinder increases. The whisker retains an amplitude greater than its VIV amplitude even at the largest distance. 144

4-15 The detectability of the whisker is higher than the other models. As the separation distance increases, the wake becomes more difficult to detect, but the whisker remains the highest. . . . . . . . . . . . 145

5-1 Pictures of the whisker-inspired flow velocity and direction sensor. . . 150

$5-2 \quad$ Picture of the re-designed whisker sensor, which was made for systematic testing in a vortex wake. . . . . . . . . . . . . . . 150 
5-3 Schematic of the slaloming trajectory (dotted line) that the whisker takes as it encounters a vortex wake. . . . . . . . . . . . . . . . . . . 151

5-4 (a) Front and (b) side views of the arrangement of whiskers as a harbor seal swims forward. Screenshots taken from supplementary video in [95].157

A-1 Digital holographic images of whiskers from a harbor seal at the New England Aquarium. All are in the $90^{\circ}$ configuration. The camera field of view and therefore the dimensions of each DH image box are $1.23 \mathrm{x}$ $1.23 \mathrm{~cm} . \ldots \ldots \ldots \ldots$

A-2 Digital holographic images of whiskers from a harbor seal at the New England Aquarium. All are in the $90^{\circ}$ configuration.The camera field of view and therefore the dimensions of each DH image box are $1.23 \mathrm{x}$ $1.23 \mathrm{~cm} . \ldots \ldots \ldots \ldots 16 \ldots \ldots$

A-3 From CT scans, these are reconstructions of (A) sea lion (B) elephant seal and (C) harbor seal vibrissae. Cross sections are enlarged and shown at 6 different locations along the span. Figure from [95] . . . . 162

A-4 Table showing measured geometric parameters of the vibrissae of these three species: harp, hooded, and grey from left to right. Figure from 46.

A-5 Table showing measured geometric parameters of the vibrissae of multiple species. Figure from [45]. . . . . . . . . . . . . . . . . . . 163

A-6 (a) Crest width and (b) trough width measured on vibrissae of different species. The diameter decreases towards the tip. Figure from [46].] . . 163

B-1 Screenshots of Model 1 and Model 2, which were designed keeping all dimensions the same as the original whisker except that one dimension of each cross section was expanded or shrunk to make it circular. . . . 166

B-2 Spiral strips combined together. The blue and red lines are the initial and final spirals. Wavelength $(\lambda)$ and the two amplitudes $\left(A_{\min }\right.$ and $\left.A_{\max }\right)$ are labeled. . . . . . . . . . . . . . . . . . . 167 
B-3 All the models created via parametric equations. From the left: Model 5, Model 4, Model 3, Model 6. . . . . . . . . . . . . . . . . 168

B-4 The twisted elliptical cylinder, as generated in Solidworks . . . . . . . 169

B-5 $C_{l, v}$ contours of Models 1 and 2. . . . . . . . . . . . . 170

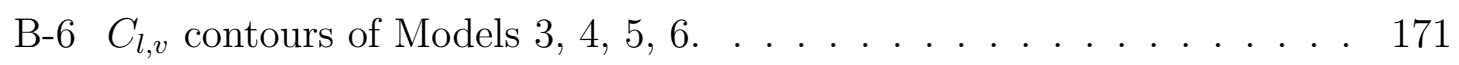

B-7 $C_{l, v}$ contours of Model 7. . . . . . . . . . . . . . . . . 171

C-1 (a) One half of the whisker mold, which was made from six blocks of machinable wax bolted together. A CNC tool path for the whisker geometry was created in MasterCAM from Solidworks and then routed out. (b) The mount to which the whisker was attached. The model was strung in the empty space pictured at the bottom. The two struts have an airfoil profile to minimize drag. The tube on top attaches directly to the front of the Tow Tank carriage. . . . . . . . . . . . . . 174

C-2 (Left) Accelerometer held in place via strings and a thin piece of plastic. (Right) Three accelerometers and two Kevlar strings inside of the mold just before casting. . . . . . . . . . . . . . . . . . . . . . 175

C-3 (a) Schematic of the setup. The whisker is held with clamps on the end. Turnbuckles are used to apply an initial tension to the Kevlar strings. The spring and pulley system on both ends allows for additional stretching as vibrations occur along the body. (b) Picture of the assembled system mounted in the Towing Tank. . . . . . . . . . . . 176

C-4 Schematic of the setup, which now includes a GoPro camera to capture motion of markers on top of the whisker at center span. . . . . . . . . 177

C-5 A calibration image. In this case a pole marked with 1in increments is used to determine the pixel to crossflow distance conversion. . . . . 178

C-6 A few of the color adjustments done to better highlight the markers. . 178

C-7 From top to bottom: crossflow acceleration, velocity, and displacement of the whisker as measured from the middle accelerometer in the $U_{n}^{*}=4$ case. . . . . . . . . . . . . . . . . . . . . . . 179 
C-8 The nondimensional crossflow (a) amplitude and (b) frequency measured both by the accelerometers and by the motion tracking software. 180

C-9 (a) Mean and (b) rms rotation of the model across different towing velocities. Both increase dramatically at high speeds. . . . . . . . . . 180 


\section{List of Tables}

1.1 Nondimensional parameters relevant to VIV. . . . . . . . . . . . . . . 62 


\section{Chapter 1}

\section{Introduction}

In the dark and murky waters, a harbor seal swims all around, searching through the turbid waters for some fish to eat for dinner. Suddenly, the whiskers on her right cheek start twitching. And she's off.

Prey had passed by several seconds earlier, and biologists at the University of Rostock's Marine Science Center [67] were clued into the harbor seals' uncanny ability to chase it. These scientists placed a blindfold and earmuffs on a seal (Figure 1-1) and found that the animal was nonetheless able to detect and track moving objects. They found evidence that the whiskers were being used to "feel" what was happening in the surrounding water, based on how they vibrate. It was already known that seals (and many other animals) can use their whiskers in a tactile manner to determine the size and shape of objects. But discovery of their potential ability to detect hydrodynamic information opens exciting new doors on our understanding of the biological world.

On the other side of the Atlantic, research was being conducted in my lab, the MIT Towing Tank Laboratory, on the vibrations of large offshore structures. For the last couple of decades, our research group has studied the physics of flow across a circular cylinder. This situation can lead to vibrations of the cylinder, a major concern for the offshore industry. Simultaneous to these studies, our group has been pioneering the field of marine biomimetics. This is where we use features observed in nature to inform the design of new marine technologies. 


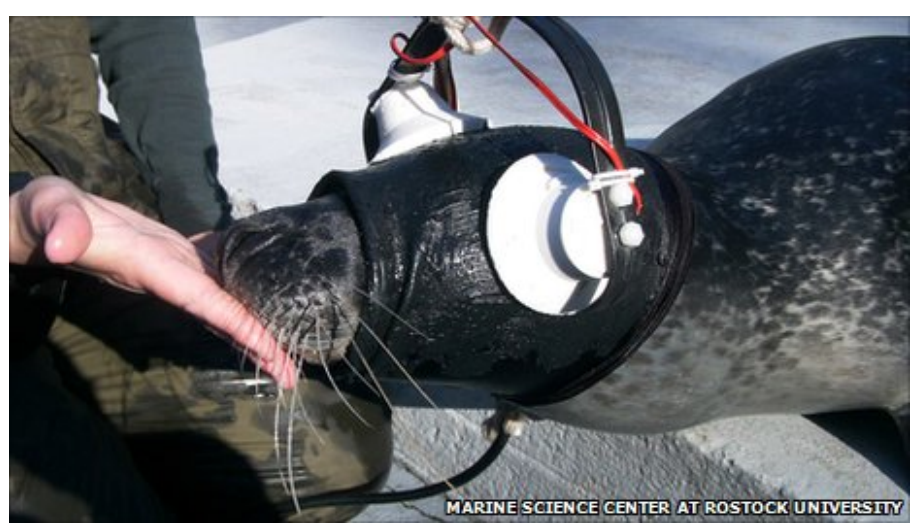

Figure 1-1: A harbor seal wearing a blindfold and earmuffs, ready for an experiment. Photo from Marine Science Center, Rostock University.

When the news of the biologists' work reached us, we jumped at the opportunity to marry these interests in vibrations and bio-inspired design. The goal on our minds, which has resulted in this thesis, was to uncover how the seals could use vibrations as sensory information. Our approach is to study the fluid mechanics of the vibrations induced by the whiskers' interactions with the surrounding flow.

\subsubsection{How might seals detect a fish trail?}

Fish leave "footprints" of a consistent pattern in the water. Each flick of their fin causes the fluid to swirl in a vortex, which lasts for many seconds after the fish is gone. Seals could reach out their whiskers to feel for these tracks (Figure 1-2).

It's surprising that this would actually work though. As our research group knows well, cylindrical objects, such as whiskers or offshore riser legs, will naturally vibrate as calm water flows over them. So how is it possible for the seal to notice that their whiskers have hit the fish tracks, if their whiskers are already vibrating a lot on their own?

The biologists in Rostock mounted a camera to a harbor seal's head in order to get a close-up view of the whiskers. What they noticed was that the whiskers did not appear to vibrate as the seal was swimming forward normally. This observation suggests that the whisker is tuned for heightened sensing, as it will vibrate minimally 


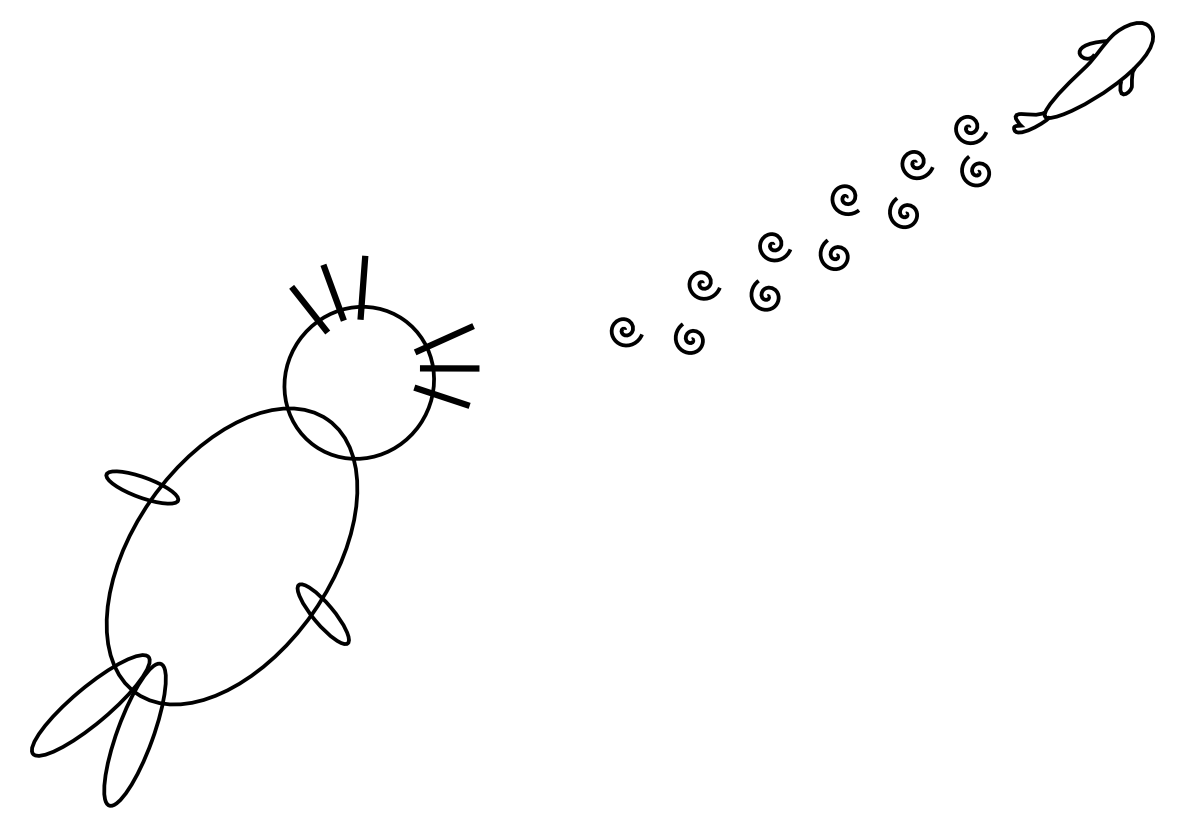

Figure 1-2: Schematic of a seal searching for a fish by using its whiskers to detect the hydrodynamic trail.

until an upstream disturbance is encountered.

In this chapter, the thesis is introduced in terms of two topics: first, the biological studies that inspire the work and second, the fundamentals of flow-induced vibrations. The remainder of the thesis takes place in the new space where these two topics combine.

\section{$1.1 \quad$ Flow Sensing}

Pinnipeds often feed in conditions with reduced visibility at night [116], in dark waters [69], even diving to depths $>300 \mathrm{~m}$ while foraging [77]. Recent behavioral experiments suggest that the reason for their success in doing so is the use of their highly sensitive whiskers to detect water movements. Their whiskers are heavily innervated with fibers that detect small deflections of the shaft. Just as rats and cats use their whiskers as a tactile sensor for active touch discrimination [31] and possibly obstacle avoidance [98], seals do so as well [23]. The study of their potential use as hydrodynamic sensors, however, has just begun.

In this section, I outline successive background context which leads up to the topic 
of the whiskers as hydrodynamic sensors: 1- an overview of all of the seals' sensory systems, 2- the structure and function of the vibrissae (whiskers), 3- some examples of related sensory systems in other animals, and 4 - the types of flow information left by fish. Then the series of hydrodynamic sensing experiments with real seals that inspire this specific study is described.

\subsubsection{Pinniped Sensory Systems}

As with many animals, the sensory systems available to the harbor seal are rich and diverse [134, 110]. These systems may enable them to carry out impressive feats observed in the field, such as precisely navigating across long distances to return to their breeding area yearly [72] and relocating breathing holes after under-ice excursions 33 .

The following section presents an overview of the known functions of each of the seals' sensory systems and how they may or may not aid hydrodynamic tracking.

\section{Vision}

The pinniped vision system is adapted for use over a broad range of light intensities. The iris is narrow and slit-like in daylight but dilates to a circular shape as ambient light decreases in the water (Figure 1-3). The large spherical lens allows for focusing of images on the retina despite the loss of refractive power under water. Harbor seals have high contrast sensitivity, which enables them to detect movement as effectively as cats [53].

Behavioral experiments with a harbor seal and California sea lion show them to have better dark adaptation rates and relative light sensitivities than a human subject. And an elephant seal, which is a deeper diving animal than the others, was measured to have an even lower threshold estimate [84].

Conditions of high turbidity are known to dramatically reduce visual acuity [136]. However, a recent experiment [47] demonstrates the harbor seal's potential to use particles in the water to resolve flow direction down to very small angles. Despite 

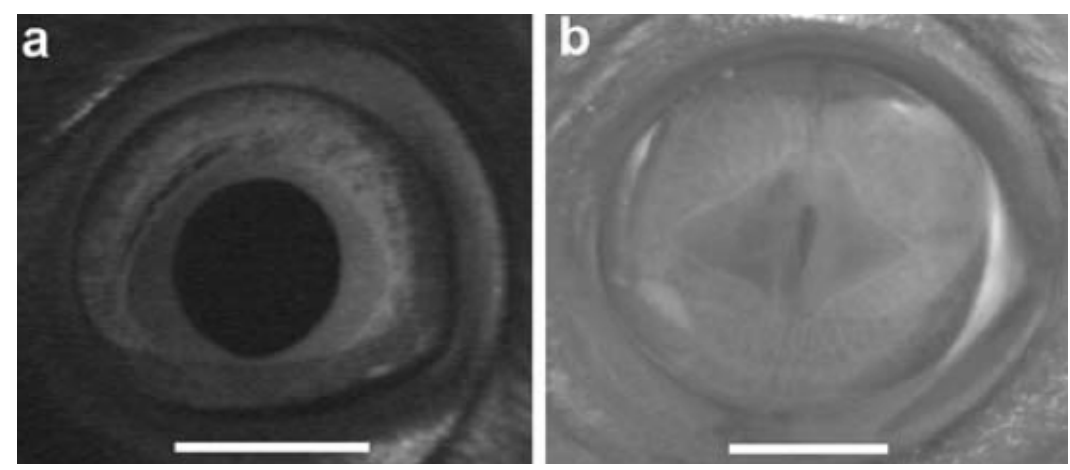

Figure 1-3: A harbor seal's eye covers a wide pupillary range to aid visual acuity in both water and air. The pupil goes from (a) circular in dark water to (b) a vertical slit on land, as light increases. The scale bar corresponds to $1 \mathrm{~cm}$. Figure from [53].

heightened visual perception, seals should only be able rely on this to locate prey when they are in relatively close proximity. The question still remains as to how these animals can hunt from long distances away.

\section{Echolocation}

Pinnipeds exhibit a range of vocalizations [116]. The pinniped auditory system, however, does not contain the same structural adaptations for localizing underwater sounds as seen in other animals (in ringed seals the auditory bulla is not acoustically isolated from the skull [69]).

Other animals, such as dolphins, are known to have an active sonar system [134], which they use to detect prey, communicate, etc. [105] observed sea lions to emit sound as they approached pieces of fish in a pool. The animals' success in catching the fish regardless of the light conditions, led the author to suggest that pinnipeds may also use an echo-ranging sense to locate prey.

Over the next few decades, multiple studies proceeded to provide conflicting evidence for pinniped echolocation. [11] again showed pinnipeds able to locate objects in total darkness. Moreover, the harbor seals here were able to discriminate between two objects, which were visually identical but differed in acoustic impedance one airfilled ring and one water-filled ring (Figure 1-4a). However, since neither the animals' sound emission nor reception were blocked as a control, reliance on echolocation in 


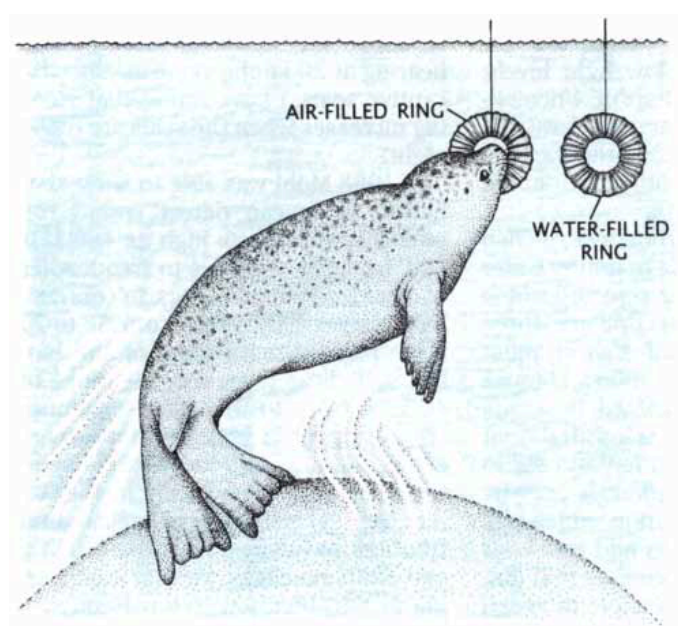

(a)

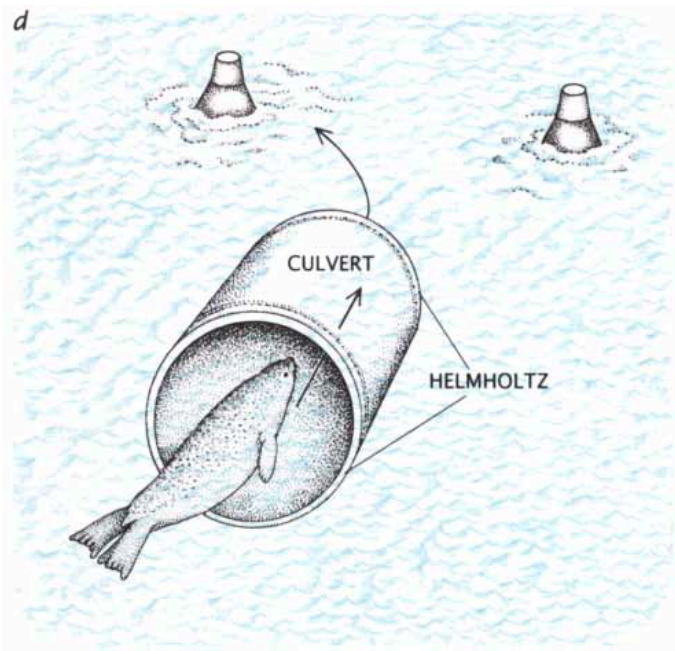

(b)

Figure 1-4: Sketches of (a) an echolocation experiment and (b) a magnetic sensing experiment conducted with harbor seals. Figure from [109].

this case could not be unequivocally stated.

On the other hand, 98] showed a negative correlation between sound emission and navigational ability, [33] recorded no vocalizations during a search for ice holes, and [18. recorded very few vocalizations by Weddell seals during under-ice prey attacks.

[119] argues against echolocation in pinnpeds, pointing out that these animals have not developed the high-frequency sound production or reception systems required for accurate underwater echolocation. Most recorded sounds in the wild were emitted at low frequencies [112], suggesting they are related to social interaction, rather than prey detection. [119] also notes that echolocation is a result of adaptation, rather than a generalized skill. Examples of opportunistic echolocation may exist, however, this does not imply that they have evolved an accurate echolocation sensory system.

The evidence against echolocation now surpasses that for it. The frequency and intensity of vocalizations in seals are low compared to those in dolphins, and their ears have no specific adaptations that allow for better functioning in the two media of water and air. It is therefore unlikely that seals would rely heavily on echolocation to detect prey. 


\section{Biomagnetism}

[109] describes a test of a harbor seal's ability to detect changes in a magnetic field. The test subject was asked to swim through a Helmholtz coil, which shifts the magnetic field (Figure 1-4b). In these test conditions, the seal exhibited poor ability to detect the change.

\section{Olfaction}

There is evidence for the olfactory system playing a role in social interactions. Its use in sensing may also be possible. There are a few demonstrations of olfactory sensations being used by animals underwater [89], including harbor seals detecting dimethyl sulfide, which can indicate profitable foraging areas [78]. However, pinnipeds are generally considered microsmatic (having a poor sense of smell) [88].

\section{Gustation}

The number of taste buds in pinnipeds is less than that on terrestrial animals, which suggests a lower sense of taste. A study by [125], nonetheless, shows harbor seals as being able to use gustation to discriminate small changes in salinity levels in seawater.

With the exception of echolocation, none of the sensory systems mentioned up to this point appear to be capable of enabling wake detection over the long distances (tens of meters) reported in multiple studies by the University of Rostock research group. The seal would need to be within close range of the prey in order to successfully use any of these.

\subsubsection{Vibrissae (Whiskers)}

General information on the vibrissal system is now provided to help determine how it may or may not be able to play a role in sensing. 


\section{Why they might be important}

Vibrissae are found in almost all mammals except Homo sapiens, and they are perhaps most prominent on pinnipeds [87]. There are several factors that point to the vibrissal system as an important one for pinnipeds. These include their number, size, growth rates, and thermal regulation system.

Adult harbor seals have about 40 vibrissae next to either nostril. Their lengths vary, but can reach up to about $125 \mathrm{~mm}$ long [117], making them among the largest known animal structures that could serve as flow sensors.

[62] and [51] measured the growth rates of harbor seal and gray seal vibrissae, respectively. The vibrissae of both species were found to not shed in the annual molt with the rest of the pelage. They are shed irregularly and individually. This suggests that seals have developed a means of ensuring continuous presence of select vibrissae for sensing.

Single vibrissal follicles are found to be units of high thermal radiation. It is suggested that by keeping the vibrissal follicle temperature constant, the vibrissal system can maintain high tactile sensitivity regardless of any decrease in ambient temperature [90, 34]. Figure 1-5 shows the elevated temperature at each individual follicle.

\section{Known functions: tactile sensing and others}

Vibrissae are known to serve multiple behavioral roles, such as communicating socially (Figure 1-6), monitoring environment parameters, exhibiting aggression, etc. [1]. It has long been suggested that mammals who must hunt nocturnally may use their whiskers to aid hunting [68], though the means by which they do this is unknown. It has also been speculated that the whiskers may serve as a filter for feeding off the ground [116].

The most known function of the vibrissae as a sensory system is that of active touch discrimination. Several studies have shown vibrissae to be sensitive to even

slight bending as they contact physical objects. [74] and [19] were the first to demon- 

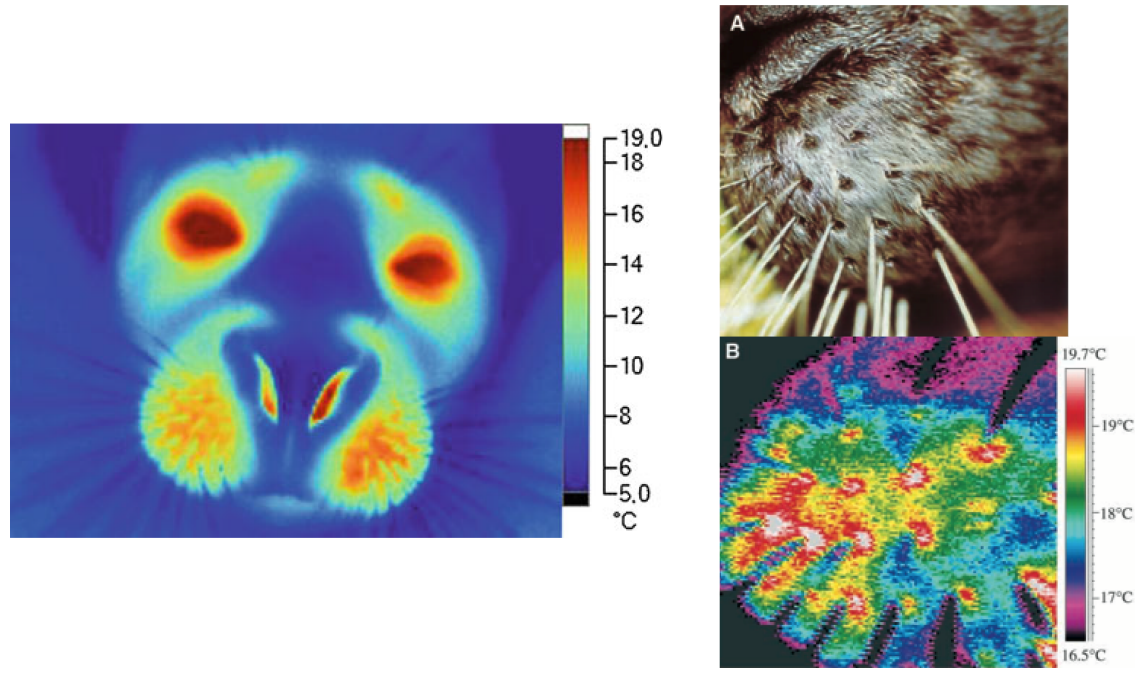

Figure 1-5: (Left) Thermogram of a harbor seal's face immediately after leaving the water. The warmest places are around the eyes, nostrils, and vibrissal pads. Figure from [21]. (Right top) Photograph and (Right bottom) thermogram of the vibrissal pad of a harbor seal Figure. The follicles have higher temperature than the rest of the pad. Figure from [90].

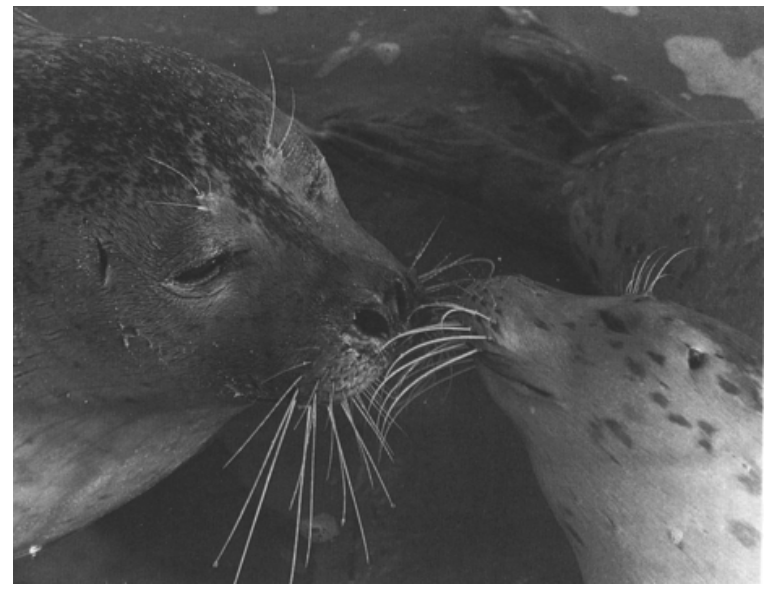

Figure 1-6: A naso-nasal greeting between a harbor seal mother and her pup, demonstrating the use of vibrissae to communicate socially. Figure from [110]. 


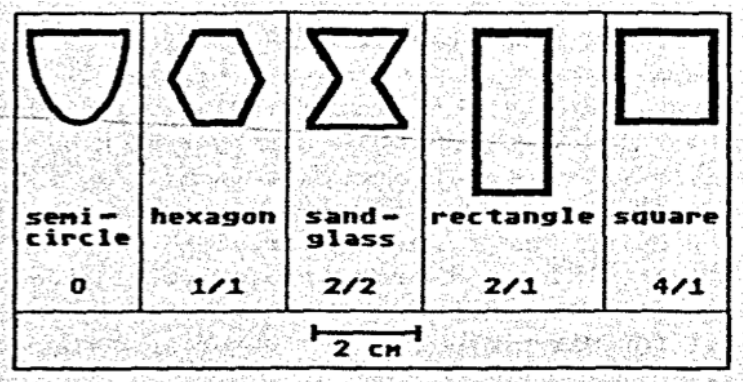

(a)

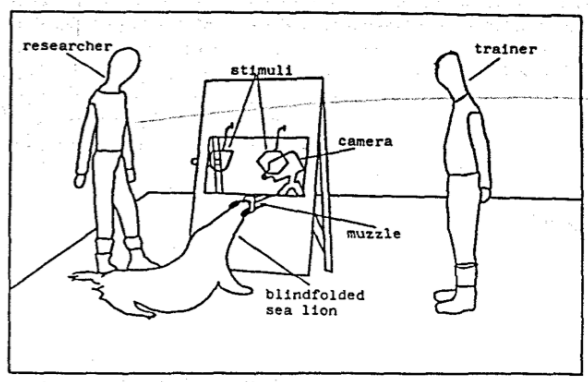

(b)

Figure 1-7: (a) Different shapes that a sea lion was asked to distinguish using vibrissal tactile sensing. (b) Experimental setup for the blindfolded sea lion. Figures from [19].

strate this tactile sensitivity in pinnipeds. Their respective experiments showed a Pacific walrus and a California sea lion able to distinguish objects of different shapes despite being blindfolded (Figure 1-7).

Next, tactile sensing was demonstrated to enable size discrimination of objects. [20] showed a blindfolded California sea lion able to identify the larger of two circular discs. The detection threshold was quantified through the Weber fraction ( $c=\Delta D / D, D=$ disc diameter $)$, which indicates the relative diameter difference detected on $75 \%$ of occasions. In these experiments, the mean Weber fraction achieved was $c=0.26$. [23] conducted the same test but with harbor seals, which belong to the family Phocidae, rather than the Otariidae (California sea lion) or Odobenidae (Pacific walrus) families. The tested harbor seals achieved a similar Weber fraction for disc diameters of the same size used in [20]. For larger discs, the Weber fraction reduced to as low as $c=0.08$. Those tests were all conducted in air, so [27] tested the harbor seals' tactual resolution ability in water. No significant difference was observed between water and air. In both media, the seals could detect size differences as low as $2 \mathrm{~mm}$.

These findings reveal the tactile acuity of harbor seals to be comparable to that of human subjects measuring the diameter of objects using their thumb and index finger $(c \approx 0.03)[43$ ] and is nearly as good as their own visual resolving power [26]. 


\section{Under the Skin: Structure}

Vibrissae are different from pelage hair in a few ways. First, the base of the vibrissae are attached to striated muscles for voluntary control [1]. Also, each vibrissa is attached to an innervated follicle, which appears to have similar structure across pinnipeds and cats [70]. In pinnipeds, however, the follicle-sinus-complex (FSC) is the largest of all mammals. They can reach $2 \mathrm{~cm}$ in length, whereas those on terrestrial mammals of similar size are much shorter (for example, those in the leopard Panthera pardus are $6.5 \mathrm{~mm}$ long) [22]. A schematic and photo of the FSC are shown in Figure 1-8. The hair shaft is encapsulated in three sections: the upper cavernous sinus, the ring sinus, and the lower cavernous sinus. The bottom two (RS and LCS) contain mechanoreceptors. The top part (UCS), which is significantly shorter or nonexistent in mammals other than pinnipeds, may serve to distance the receptors from low ambient water temperatures [69, 90]. No nerves are present inside the hair shaft. Rather, at the base of the FSC, a nerve bundle penetrates into the capsule. This leads away to a specific location in the brain's sensory cortex.

In [79], different parts of a fur seal were stimulated while the activity of cortical neurons was recorded via microelectrodes inserted in the somatosensory cortex. Excitation of the vibrissae was found to stimulate a large portion of the somatosensory cortex (Figure 1-9). The area occupied by projections of the head are greater than the area occupied by the trunk and limbs combined. It is well established that the relative size of projections indicates the importance of the tactile information received from those zones. At the time of that study, no other equal to this vibrissal representation had been seen in other mammals. Also, despite the variation in size of vibrissae, they all take up approximately the same area in the cortex, suggesting that each is equally useful.

The nerves penetrating the FSC in pinnipeds have an order of magnitude higher innervation than those in terrestrial mammals $(1,000-1,500$ nerves to each vibrissae in ringed seals versus 100-120 in beavers and rats) [71]. The same comparison can be made with the mechanoreceptor density in the ring sinus of the FSC, where the 


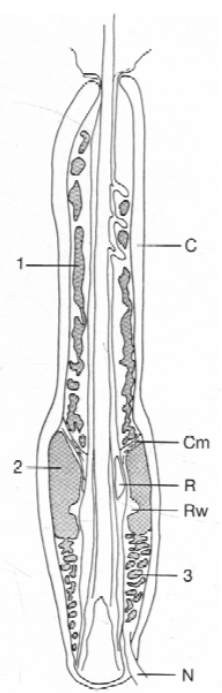

(a)

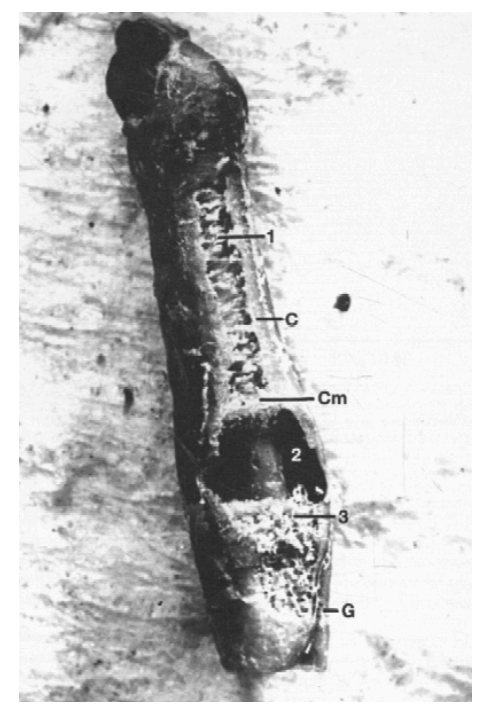

(b)

Figure 1-8: (a) A diagram of the follicle-sinus-complex system, which holds each vibrissa and connects it to the brain. (b) A ringed seal's FSC partially split open. 1: upper cavernous sinus, 2: ring sinus, 3: lower cavernous sinus, C: capsule, Cm: collagenous membrane, R: liquid-filled cleft, $\mathrm{Rw}$ : ringwulst, $\mathrm{N}$ : nerve bundle penetrating the capsule, G: groove. Figures from [69].
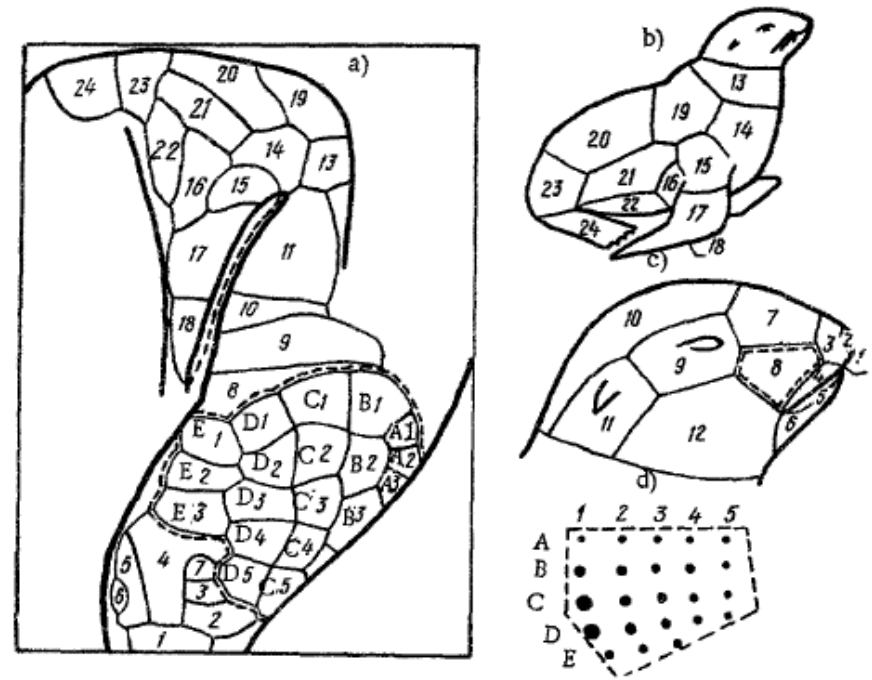

Figure 1-9: (a) The somatosensory cortex portion of the brain.

The points on the body that stimulate the labeled regions on the cortex from (b) the trunk, (c) the head, (d) the vibrissae (marked in size according to length and diameter). The vibrissae are seen to take up a sizable portion of the total area. Figure from [79]. 
number of Merkel cells in a ringed seal's ring sinus is 2-5 times greater than that in cats [69, 70]. The increased innervation density again suggests an important sensory role for pinniped vibrissae.

\section{Under the Skin: Nerve Response}

The term vibrissa comes from the Latin word "vibrio", which means to vibrate. Studies have been carried out to determine how the nerve fibers respond to vibrations, as that will define the scope within which vibrissae can serve to relay sensory information.

These nerves are composed of afferent (indicating direction is from the receptor to the central nervous system) fibers that respond to deflections of the hair shaft. Two classes of afferent fibers are distinguished: slowly adapting (SA) and rapidly adapting (RA). The former discharges throughout the duration of a stimulus. The latter provides a nerve impulse only during a movement and therefore does not respond to steady displacement. It is assumed that SA fibers provide position information and RA fibers provide vibration information.

[30] and [31] attempted to identify the range of stimuli that produce neural activity in the afferent fibers. The author stimulated individual afferent fibers on vibrissae of harbor seals and cats, and he surgically exposed the infraorbital branch of the trigeminal nerve to measure the response. Seals were found to have a somewhat higher percentage of RA fibers than cats. Otherwise, the two species showed minor functional differences. Their results suggest that harbor seals are capable of detecting vibrations between $5-700 \mathrm{~Hz}$ and with a minimum threshold of about $10 \mu \mathrm{m}$. For some fibers, the discharge frequency was unaffected by the stimulus amplitude. In both species, the response of the SA fibers saturated between $12-20^{\circ}$ of vibrissae deflection, indicating their sensitivity to minor hair deflections, rather than large ones. The RA fibers showed strong ability to detect small vibratory disturbances. The author notes that these properties should aid tactile detection of texture and shape.

Later studies [107, 92] measure the smallest amplitude that seals may perceive while their vibrissae are vibrating (in the range of 50-1000 Hz). Harbor seals were 


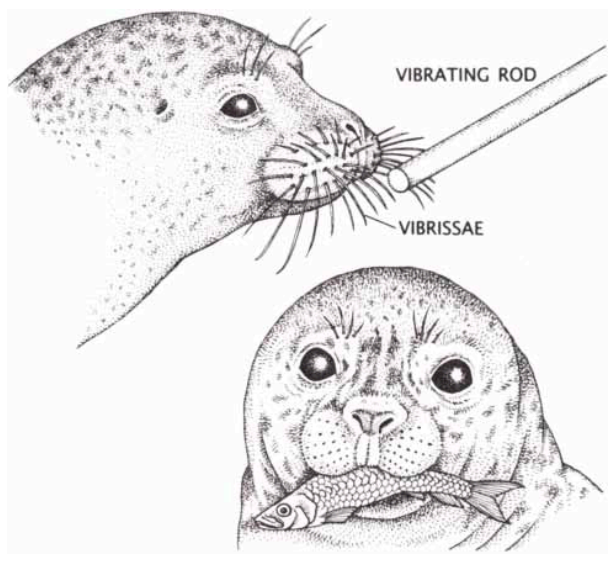

Figure 1-10: Sketch of experiment, in which a harbor seal was asked to identify whether or not a rod in contact with its vibrissae was vibrating. Figure from [109].

trained in air to touch their whiskers to a rod (Figure 1-10) and indicate whether or not the rod was vibrating. Unlike the previous studies, these results indicate higher sensitivities at higher frequencies and an ability to detect displacements $<1 \mu \mathrm{m}$.

The natural frequencies of whiskers shed by fur and harbor seals were measured [121, 59]. They show a first mode of vibration at around $40-60 \mathrm{~Hz}$. This is on the lower end of the spectra tested above. The question of interest for wake following would be whether or not the vibrissae are sensitive to the low-frequency signals that would be left in their prey trails $(O(1) H z)$.

\section{Morphology}

Most phocid seals exhibit a different whisker morphology than other pinnipeds [86]. Elephant seals (Mirounga spp.), gray seals (Halichoerus grypus), harbor seals (Phoca vitulina), harp seals (Phoca groenlandica), hooded seals (Cystophora cristata), and ringed seals (Phoca hispida), have an undulatory and flattened shape. Other pinnipeds, such as bearded seals (Erignathus barbatus), monk seals (Monachus spp.), those in the Odobenidae family (walruses), and those in the Otariidae family (fur seals and sea lions) have a smooth profile. Sketches of the different morphologies are shown in Figure 1-11 and an image comparing the ringed seal's and sea lion's whiskers is shown in Figure 1-12.

In [46] the bumpy morphology of the vibrissae on harp, hooded, and grey seals were 


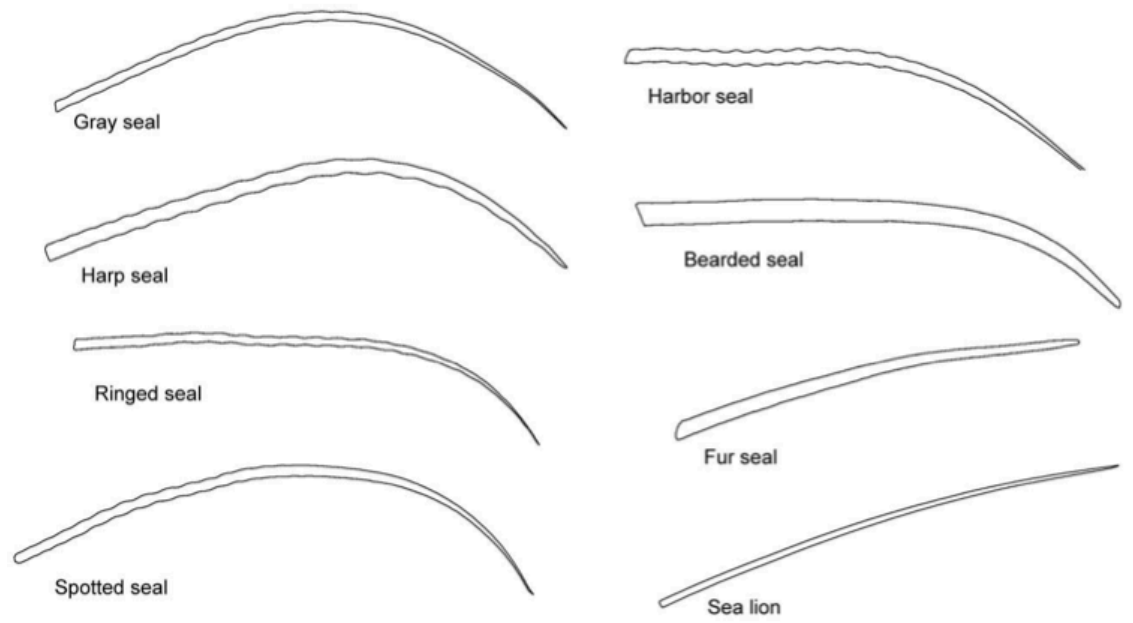

Figure 1-11: A few of the different vibrissal morphologies seen in pinnipeds. Phocid seals generally exhibit an undulatory profile unlike the smooth profile seen on otariids. Figure from [45].

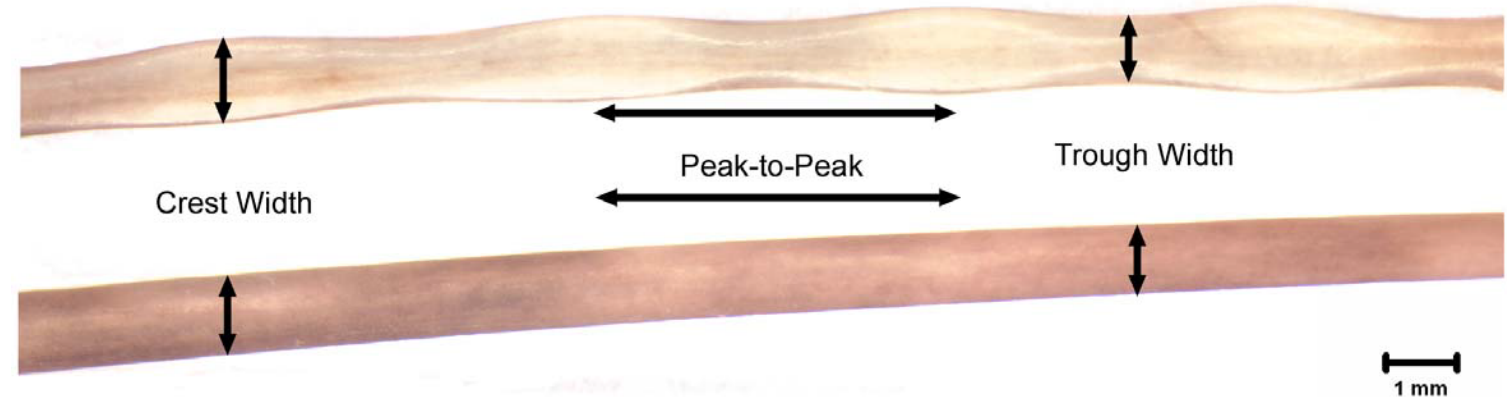

Figure 1-12: Pictures of a vibrissa from a ringed seal (top) and California sea lion (bottom). Figure from [45]. 


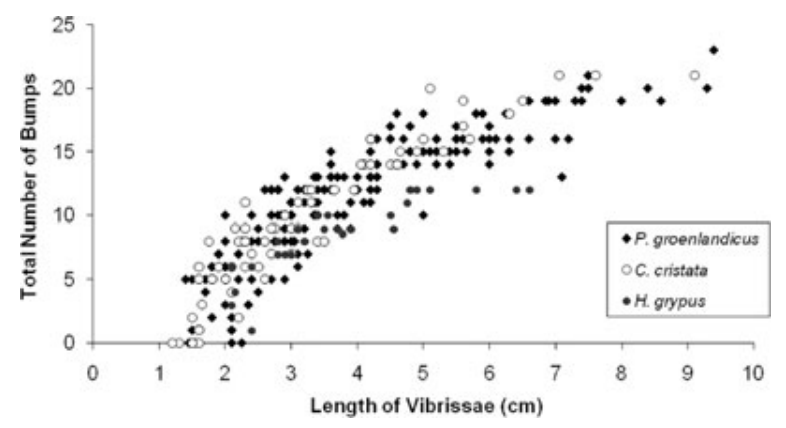

Figure 1-13: The number of bumps is seen to increases somewhat linearly with vibrissa length. Figure from [46].

optically measured. The bearded seals' smooth vibrissae were used for comparison. The wavelength of the bumps is relatively constant, as the number of bumps increases fairly linearly with the total whisker length (Figure 1-13). From their data, the $\lambda / d$ ratio is about 5 to 6 , where $\lambda$ is the undulation period and $d$ is the average diameter on the long side of the whisker. They note that the shortest vibrissae (length $\leq 25 \mathrm{~mm}$ ) had no bumps, indicating that there is some development aspect to the bumpy morphology. Also, the width of the vibrissae tapers towards the tip.

In [45], morphological differences between phocids with the beaded vibrissal profile, phocids with a smooth profile, and otariids with a smooth profile were compared. Using a combination of traditional and morphometric analyses, $100 \%$ of vibrissae were able to be classified according to the species. It is therefore shown that these three groups occupy distinct morphospaces (based on peak-to-peak distance, crest width, and trough width).

The harbor seal's vibrissal geometry is described by [45] as having, on average, a peak-to-peak distance of $3.3 \mathrm{~mm}$, crest width of $0.9 \mathrm{~mm}$, trough width of $0.73 \mathrm{~mm}$, total length of $86.5 \mathrm{~mm}$, and 2.3 beads $/ \mathrm{cm}$. The three-dimensionality is described in [58], though they report a significantly shorter undulation wavelength than that in [45]. The latter matches what I measured from vibrissae provided by the New England Aquarium.

A variety of foraging methods are employed across these species. It is therefore conceivable that their vibrissal morphologies could have evolved to serve slightly dif- 
ferent functions. Otariids generally do not hunt in as deep water as phocids do, and therefore should not need to rely as much on non-visual cues. Likewise, their vibrissae perhaps need not have developed the extra feature of undulations, as those on phocids have.

\subsubsection{Other Animals}

Several other animals, which have similar systems to the pinniped vibrissae, will now be briefly overviewed.

\section{With hairlike structures}

Many marine mammals have a vibrissal system [87]. Baleen whales have about 100 vibrissae around their blowholes and jaw. Odontocetes (dolphins, etc.) have vibrissae, but most lose them postnatally. Manatees have vibrissae across their body and have been shown capable of detecting hydrodynamic dipole stimuli using those on their face [106, 42]. They are herbivorous, however, so it is less likely that they would need to employ wake tracking. Several terrestrial mammals also have vibrissae (cats, rats, insects, etc.). Studies on some of these have shown their tactual function [38], and have inspired creation of artificial tactile sensors [122]. Figure 1-14 displays a few animals with vibrissae.

There are also animals with non-vibrissal structures, which are nonetheless hairlike and can be used for sensing (Figure 1-15). For example, moles, shrews, and aquatic snakes conduct tactile sensing using such structures [15, 14]. Also, the lateral line organ on fish consists of a set of neuromasts. These are a group of thousands of sensory hair cells with kinocillia and stereovilli that project into a flexible cupula (tens to hundreds of micrometers in size). Deflection of this structure provides sensory information to the fish. 


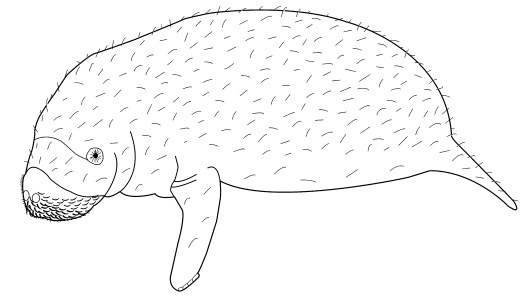

(a)

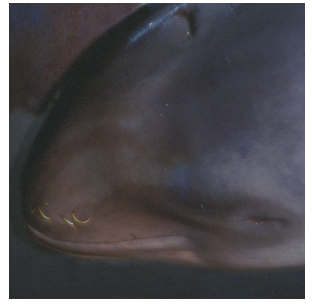

(b)

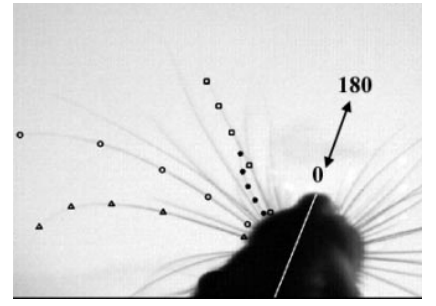

(c)

Figure 1-14: Examples of other animals with vibrissae: (a) manatee (figure from [106]); (b) harbor porpoise (figure from [16]); (c) rat (figure from [61]).

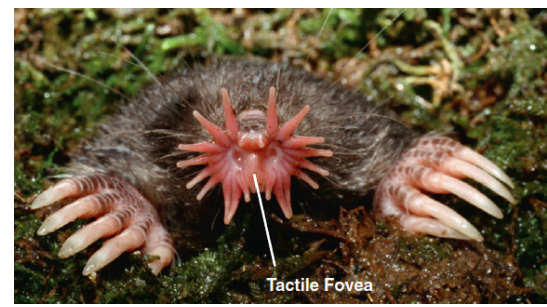

(a)

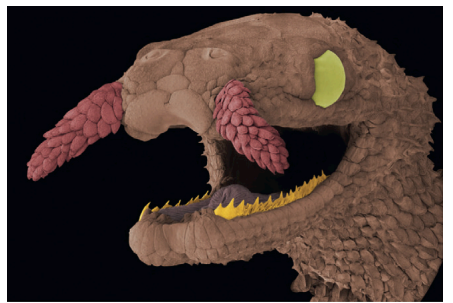

(b)

Figure 1-15: Examples of other animals with hairlike structures: (a) star-nosed mole and (b) tentacled snake (both figures from [14]).

\section{Other types of sensing}

There are many examples of non-vibrissal based sensing in animals. A few are listed here to give a sense of the vast range of sensory capabilities in the animal kingdom. Bloodhounds and other dogs use olfaction to detect the direction of a track [128]. Copepods can conduct chemical sensing. Snakes can use their tongues to transport odors and follow scent trails [120, 135]. Sharks can detect electric fields [73]. The starnosed mole and water shrew are able to sniff and detect semiochemicals underwater [89]. Crocodiles have dome-shaped pressure receptors distributed across their face.

\section{Other instances of tracking}

There are a limited number of studies of animals conducting wake tracking. One of the few examples is that of a catfish tracking a small guppy. The test subject's vision was obscured by shining infrared light [104] and its taste sensation was ablated [103]. Ablation of taste was not seen to lower the effectiveness of guppy capture, but 


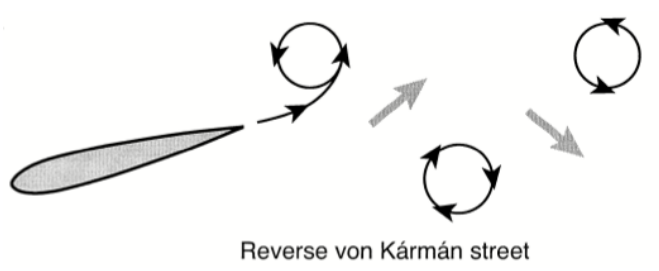

Figure 1-16: Sketch of the reverse von Kármán vortex wake left by a swimming fish, which produces thrust. Figure from [29].

restriction of the lateral line did. At first glance, the prominence of their "whiskers" suggest they could play a role in hydrodynamic sensing. However, these appendages have taste buds, so are likely used in chemical sensing. Also, these barbels are fleshy, unlike the stiff vibrissal hair shafts, which sit in an innervated FSC. It should be noted that this tracking was done over relatively short distances (50 guppy lengths) and times $(10 \mathrm{sec})$, which still leaves the performance of the harbor seal as a yet unrivaled and unexplained feat.

\subsubsection{Hydrodynamic Stimuli}

The focus of this thesis revolves around detection of hydrodynamic information. This section provide details on what information may be left in the wakes of swimming animals for the seals to sense.

The hydrodynamic trails produced by a swimming fish can take many forms, depending on various parameters that define their kinematics (pitch angle, heave amplitude, flapping frequency, etc.). It is beyond the scope of this thesis to describe multiple types of wakes and study seals' responses to them. Instead, I chose to focus on the type of wake left during efficient swimming, which is that of the reverse von Kárman vortex street (Figure 1-16). A vortex is shed with each flick of the fin, which results in an array of counter-rotating vortices and a mean flow that produces thrust.

Studies by [55] and [54] provide some information on how long fish wakes can last. In [55], even the wakes of small (10 cm long) goldfish (Carassius auratus) could still be measured 5 min after the fish swam by in the low background flow lab conditions of the study. [54] measured the trails of three teleost species (38-86 $\mathrm{mm}$ long). Typical flow 
velocities measured $60 \mathrm{sec}$ after the wake generating fish had passed were $12 \mathrm{~mm} / \mathrm{s}$. The authors state that this implies that a predator could detect the fish at a distance of tens of meters. A harbor seal itself was measured to leave flow velocities of at least $30 \mathrm{~mm} / \mathrm{s}$ after $30 \mathrm{~s}$ [118]. Based on extrapolated values, [97] proposed that water movements caused by the C-starts of teleost fish may last for several minutes, even if background noise is present.

An efficiently-swimming fish will flap its tail at a nondimensional frequency described as the Strouhal number $(S t=f w / U)$ of $\sim 0.3$, where $w$ is the wake width, $U$ is the swimming speed, and $f$ is the flapping frequency [129]. The size and speed of fish pursued by harbor seals should therefore produce vortex wakes with dominant frequencies of $O(1-10) H z$.

\subsubsection{Hydrodynamic Perception Experiments with Pinnipeds}

In the 1950s, and perhaps earlier, it was suggested that seals may use their vibrissae to detect water changes as a cue for prey detection [60]. A field observation of a blind grey seal, who was nevertheless fat and healthy, indicated its ability to catch food. The author ruled out the possibility of sight, smell, and hearing and concluded that its vibrissae must have been used: "One may suppose that by means of them [vibrissae] the animal is made aware of slight turbulences in the water such as might be produced by the swimming of a fish."

Since the 1990s, the Marine Science Center group in Rostock has led a series of experiments with pinnipeds, demonstrating, in particular, harbor seals' ability to perceive hydrodynamic information. A review of these experiments has been captured in a few places [57, 143, 56, 21] and an overview is provided here.

\section{Dipole Stimuli Detection}

[24] was the first behavioral test of a seal's ability to use its whiskers to detect hydrodynamic stimuli. A similar setup as has often been used to test the lateral line sensing capabilities of a fish was employed here (Figure 1-17). An oscillating sphere 


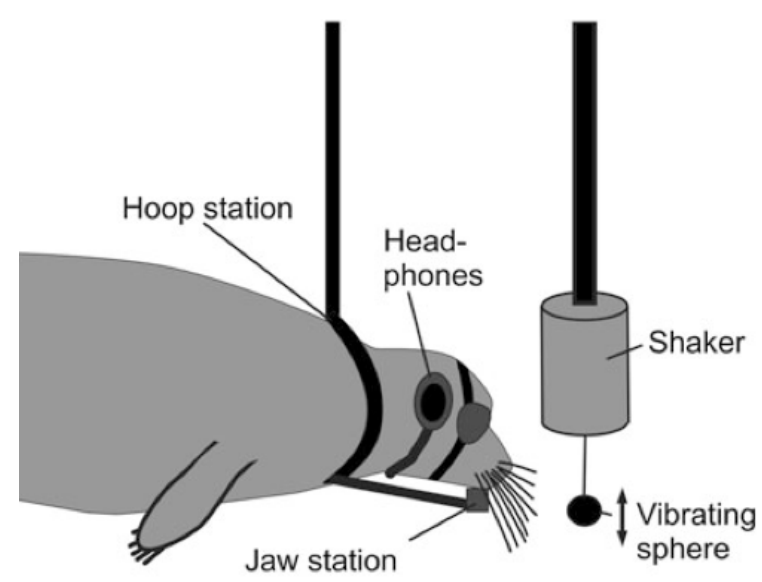

Figure 1-17: Experiment setup for dipole stimuli detection. A harbor seal wearing a blindfold and earmuffs was stationed at a set distance from an oscillating sphere. Vibrissal detection threshold of the resulting water movements was measured. Figure from [26].

generated a hydrodynamic stimulus over a range of $10-100 \mathrm{~Hz}$. The seal was trained to rest its jaw on a station to maintain a specific distance between it and the sphere. Optical and acoustic cues were removed by using eye caps and headphones with pink noise masking. The seal was trained to leave the hoop station upon detection of the stimulus.

The distance between the stimulus and the whiskers was adjusted and used to calculate an effective stimulus amplitude. The best response was seen at $50 \mathrm{~Hz}$, which corresponded to $\sim 250 \mu \mathrm{m} / \mathrm{s}$. At that frequency, the absolute detection threshold of water displacement was $0.8 \mu \mathrm{m}$. The seal was also tested while wearing a wire mesh muzzle, which blocked whisker movement. In those cases, he never responded to the stimuli. These results indicate the harbor seal's ability to detect minute movements using its whiskers, though the detection threshold is not as sensitive as that seen in some fish species.

\section{Toy Submarine Trail}

Next, seals' ability to follow the trail left by a moving object was demonstrated [25]. The authors selected a propeller-driven toy submarine of a size that should create trails with similar flow velocity and spatial extent similar to those wakes of 


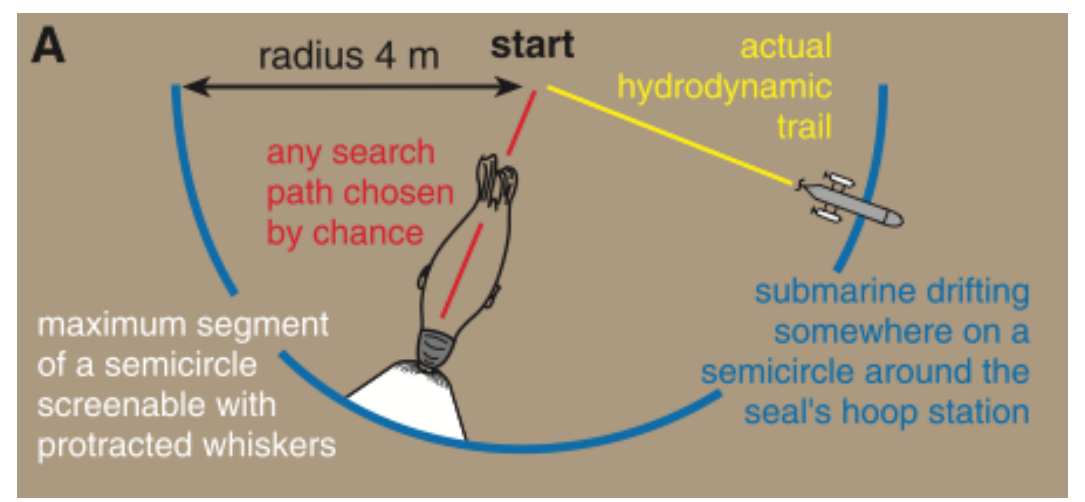

Figure 1-18: Schematic of the submarine trail detection experiment. A harbor seal wearing a blindfold is asked to locate a toy submarine, which swam ahead on a linear path in a random direction several seconds earlier. The yellow line marks the actual trail, the red line indicates a potential search path the seal could've taken, and the blue semicircle marks the locations where the sub could have landed. The probability of the seal finding the submarine by arbitrarily choosing a direction is $P \leq 0.04$. Figure from [25].

swimming fish measured in [55]. Again the seal was blindfolded by wearing a stocking mask. Headphones were used for acoustical masking while the submarine swam ahead (for 3-5sec). About two seconds after the submarine stopped, the headphones were removed and the seal began its search.

The sub was commanded to follow a linear path in a random direction (Figure 1-18). The seal began its search by protracting its vibrissae to the forward position and slightly moving its head laterally until it detected the trail, at which time it swam towards the submarine. Even with a delay (up to 20sec) between starts of the two objects, the seal successfully found the submarine $\sim 80 \%$ of the time. The authors note that this $20 \mathrm{sec}$-old wake is equivalent to the seal having chased the object from $\sim 40 m$ away.

A spontaneous change in course was then introduced into the submarine's path. As the seal encountered this change, it sharply turned to stay on the trail. On the other hand, if the engine was allowed to run for a short time after the earmuffs had been removed, the seal would follow a direct path to the source (Figure 1-19). Finally, an experimentally naïve harbor seal was able to carry out these tasks with similar performance, further solidifying evidence for the harbor seal's hydrodynamic 


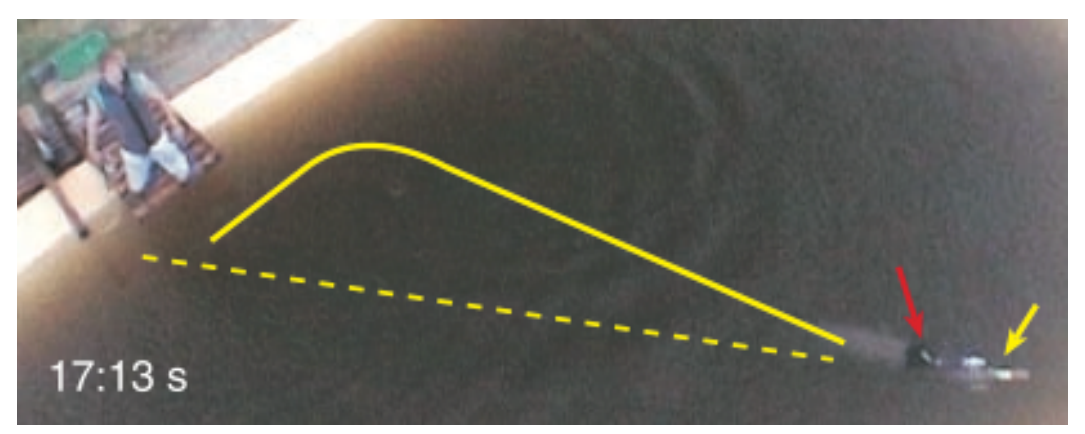

Figure 1-19: The two paths taken by the harbor seal (red arrow) to locate the toy submarine (yellow arrow). In the absence of acoustical cues, the seal followed the sharp turn that the sub also took (solid yellow line). When the sub engine was audible, the seal followed a direct path to it (dotted yellow line). Figure from [25].

perception.

\section{Biogenic Trail}

[118] showed the accuracy and success of trail following to be even slightly higher (success rate $\sim 90 \%$ ) when the seal followed a second seal (biogenic tracking). A similar experimental setup was used, with the test seal wearing a blindfold and earmuffs until the wake generator reached its destination.

Trail generation lasted a mean of $13.2 \mathrm{sec}$. The blindfolded seal was able to closely follow the path of the front seal, never deviating more than one body length from it. It was seen to employ two types of search patterns: in $\sim 65 \%$ of the cases, it followed a linear pattern and in the remaining $\sim 35 \%$ of the cases, the seal repeatedly crisscrossed the path (Figure 1-20). The authors postulate that this may be related to the angle at which the seal approached the wake: At acute angles, it could stay head-on with the trail; from more obtuse angles, it may have overshot and needed to correct. Control experiments, where the seal wore a stocking mask muzzle, were unsuccessful, as the mask irritated the seal and he refused to perform the search.

\section{Artificial Fin Trail}

Then [138] tested a harbor seal's ability to determine trail direction, based on the wake left by a finlike paddle. Two paddles were placed at either end of an enclosed 


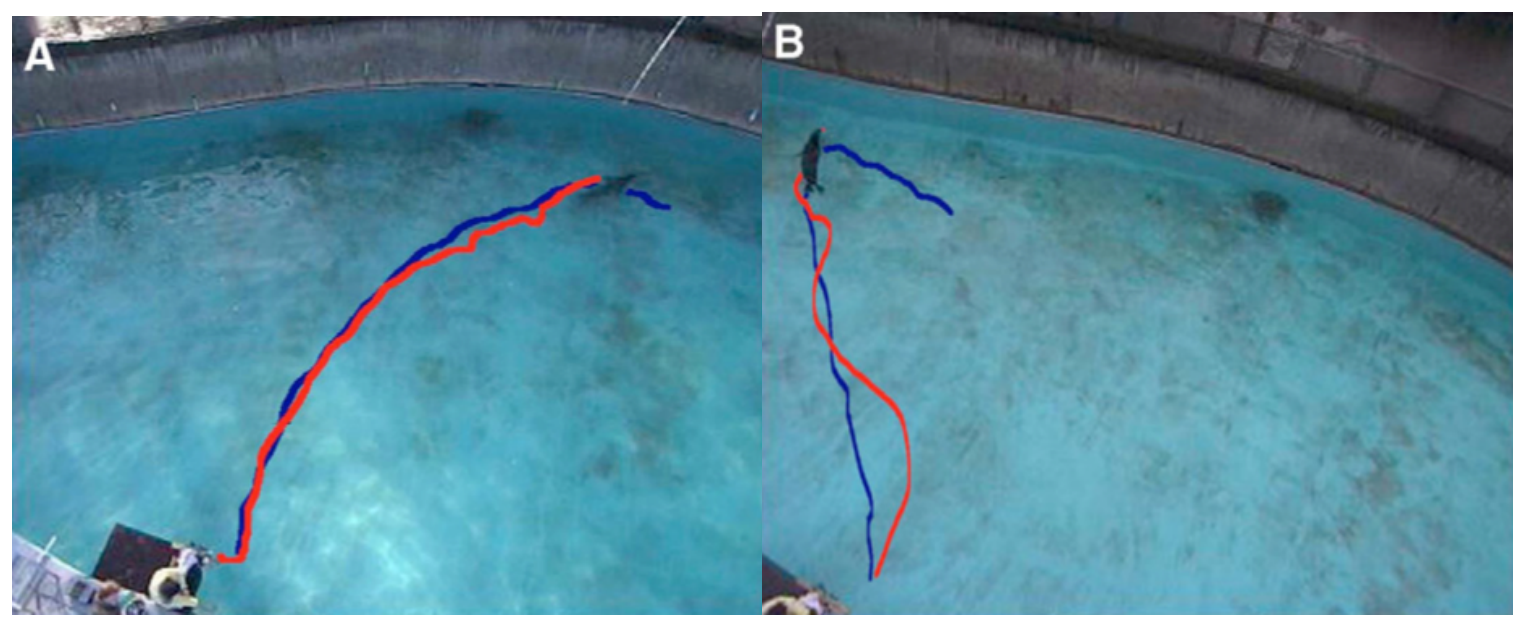

Figure 1-20: (A) One seal swims ahead on the blue path. A second seal swims behind several seconds later and takes the red path, despite being blindfolded. (B) Same setup, but the second seal now employs a more distinctively undulatory pattern to the search. Figure from [118].

tank. The experimenter moved one across the tank $(U=0.2 \mathrm{~m} / \mathrm{s})$, and when the paddle reached the other end, the seal had to identify which direction the movement had been in. Based on a metric of $70 \%$ correct choices, they show the seal is able to detect this motion up to $35 \mathrm{sec}$ after the wake was created.

It should be noted that a rigidly clamped fin does not generate a reverse von Kármán vortex street, as a swimming fish does. So the manner in which the seal determined direction in this study may not match that used by seals chasing fish. Based on the information presented so far, it is difficult to say how the specific flow structures may have aided detection. In this case, and in the previous two tracking studies, it is possible that the difference in the mean flow velocity of the wake compared with the ambient flow was sufficient information for the seal to determine the direction of the wake-generator.

\section{Impact of Glide Phases}

Fish often do not swim continuously, rather they perform alternating burst and glide phases, which may improve swimming energetics. [139] tested the impact this type of swimming would have on seals' detectability of the wake. A submarine was com- 


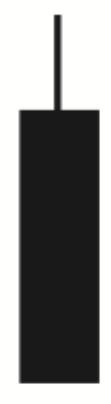

I

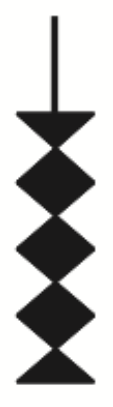

II

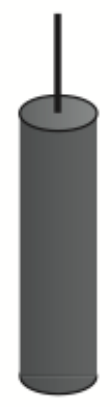

III

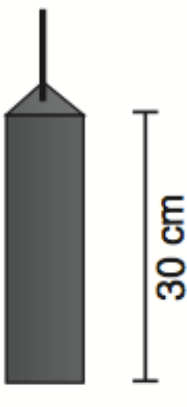

IV

Figure 1-21: Different shapes that a seal was trained to distinguish based solely on the wake left while they were held fixed and towed. Figure from [140].

manded to run continuously for 3-5m then glide for another $3-5 m$. The seal was able to track this wake with about the same reliability as a continuous wake, unless the delay was longer than $15 \mathrm{sec}$.

\section{Object Size and Shape}

[140] showed the seals' ability to distinguish certain sizes and shapes of objects based on their hydrodynamic signature. Chemically inert paddles of different shapes (Figure 1-21) and sizes were towed in front of a blindfolded seal. There was a three-second delay between the models reaching their endpoint and the seal being allowed to enter the tank.

Based on the wake left by these different objects, harbor seals are able to detect a difference in object sizes of at least $4 \mathrm{~cm}$. This is not as sensitive as the fish's lateral line detection in terms of dimensional value [132, however it would likely be sufficient resolution for seals, whose normal prey size is at least $10 \mathrm{~cm}$ in length [12].

Again, it is difficult to say what specific pieces of information from the wake the seal may have used in this case to conduct detection.

\section{Comparison with Sea Lion}

[48] provides the first behavioral evidence that the shape of the whisker may play a role in hydrodynamic detection. Using similar experimental conditions as in [25], a 
miniature submarine was released ahead of the blindfolded test subject. This sub was remotely controlled and commanded to take various paths with curves.

In this case, the experiment was conducted on a California sea lion, which has a different vibrissal morphology than that of the harbor seal. The former has a smooth profile and the latter has a "beaded" structure as described in Section 1.1.2.

The success rate $(\sim 85 \%)$ was very similar to that of the harbor seal in the previous study, and the test subject again followed any curves present in the path. However, unlike the harbor seal, if the sea lion had to wait more than $5 \mathrm{sec}$ after the submarine had left, it mostly lost the trail.

An individual's performance does not necessarily represent that of an entire species, however these results may suggest that sea lions' reduced trail-following ability compared with harbor seals may be related to their different whisker morphologies.

\subsubsection{What is the mechanism of detection?}

These demonstrations naturally raise the question of how seals can do this. What enables harbor seals to detect these trails using their whiskers alone? As noted in [84], it is understandable that high vibrissal sensitivity could enable heightened tactile sensing, which can be used for "sit-and-wait" foraging strategies or burrowing in the ground. But how can the vibrissae be used while the seal is actively chasing prey? The wakes left by prey should be difficult to detect above background noise while the seal is swimming.

This is the question explored in this thesis. A key hint was revealed in [58], which showed a potential connection between vibrations and sensing. The following section provides background on vortex-induced vibrations, which are the underlying physics that will help reveal the answer. 

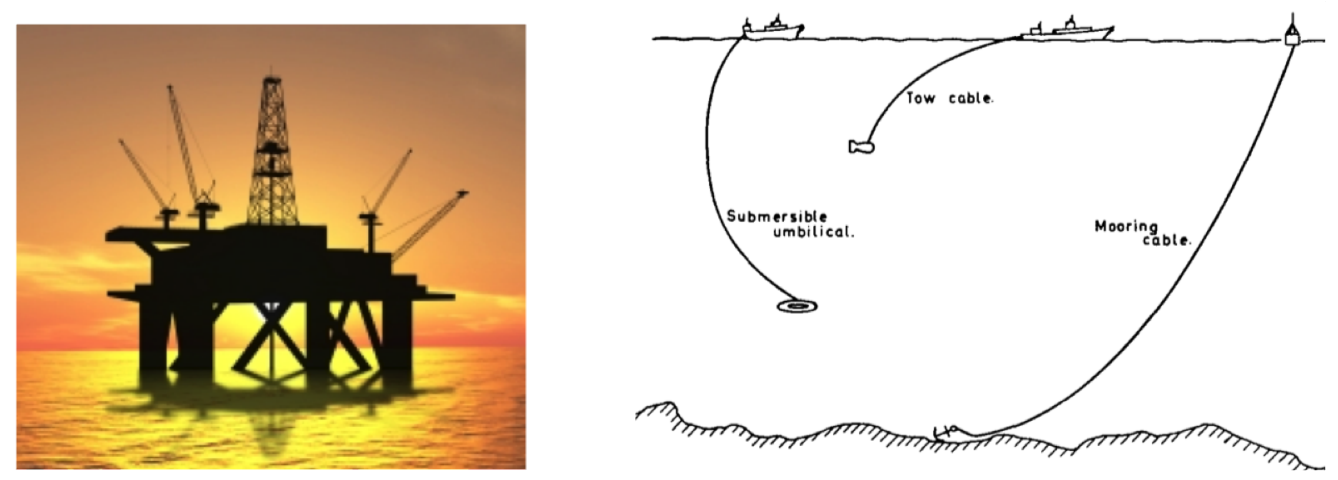

Figure 1-22: Ocean structures, such as offshore platforms (figure from [66]) and cables (figure from [35]), are subject to VIV.

\section{$1.2 \quad$ Vortex-Induced Vibrations (VIV)}

As a cylindrical object, such as a vibrissa, moves through steady flow, it can develop significant vibrations. This phenomenon, known as vortex-induced vibrations (VIV), has been studied for decades and several reviews have been written [8, 114, 142]. Despite extensive studies, this phenomena continues to present serious challenges in engineering applications, such as design of large-scale ocean structures (Figure 1-22), which will experience structural fatigue after prolonged vibrations.

\subsubsection{Why does VIV occur?}

As flow goes over a circular cylinder, vortices are shed. These exert a force back onto the object. If the natural frequency of the cylinder is sufficiently close to the vortex shedding frequency and if the cylinder damping is low enough, the cylinder will undergo sustained oscillations.

\section{Vortex Shedding}

Bluff bodies are geometries that have a cross-section with a span-to-chord ratio of about 1. A circular cylinder is the canonical example bluff body, and it will be used throughout this chapter. When a steady flow encounters this type of shape, the fluid is forced to deviate from its uniform path and it attempts to stay attached to the cylinder surface. Reynolds number is defined in equation 1.1 , where $U$ is the free 
stream flow velocity, $d$ is the cylinder diameter, and $\nu$ is the kinematic viscosity of water.

$$
R e=\frac{U d}{\nu}
$$

When $R e$ is $>40$, the flow separates from the back half of the cylinder and peels off into swirling vortices. This vortex shedding happens in an alternating pattern that creates a turbulent wake known as the von Kárman vortex street.

This street, or wake, is composed of periodically shed vortices with alternating rotation. The nondimensional parameter used to describe this flow is the Strouhal number $(S t)$ and is defined in Equation 1.2 using $f_{s}$ as the vortex shedding frequency.

$$
S t=\frac{f_{s} d}{U}
$$

This value is a function of Reynolds number [1], but for a large range of subcritical Reynolds numbers, it remains relatively constant at $\sim 0.2$ for a circular cylinder.

\section{Fluid-Structure Interaction}

Vortex shedding can have a significant effect on the cylinder itself. If the cylinder is either flexible or flexibly mounted, its natural frequency can be excited by the vortex shedding process. When these two frequencies are close to each other, the cylinder may undergo large amplitude motions.

This is because a vortex is a low-pressure region, which exerts a force back onto the cylinder. So as vortices are shed, the cylinder experiences an oscillatory transverse force. This transverse force corresponds with the vortex shedding frequency, which can change to synchronize with the motion frequency. This is called lock-in. The fluid and the structure operate in a feedback loop, since the body force increases the strength of the vortices, which in turn increases lift and drag on the cylinder. This results in high oscillation amplitudes. 


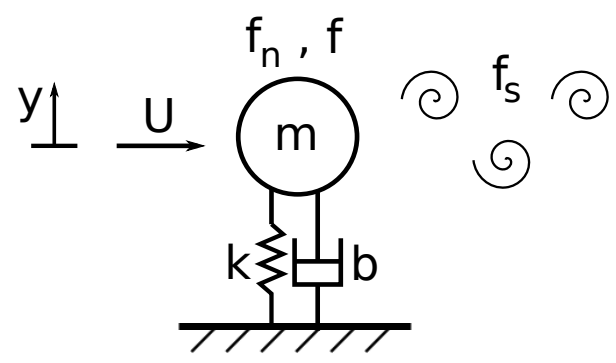

Figure 1-23: A bluff body in a uniform flow can be simply represented as a 1-degree-offreedom linear oscillator. The free stream approaches with velocity $U$ causing vortex shedding at a frequency of $f_{s}$.

\subsubsection{Model}

An elastically mounted bluff body in a free stream can be simply represented as a 1-D harmonic oscillator. A representative mass-spring-damper system that is free to move in the transverse (crossflow) direction with position $y(t)=y_{0} \sin (\omega t)$ is shown in Figure $1-23$.

The cylinder has structural mass $m$, structural damping $b$, and spring constant $k$. The motion occurs in the $y$ direction, and the fluid force on the cylinder in this direction is given by $L$. The governing equation is shown in Equation 1.3.

$$
m \ddot{y}+b \dot{y}+k y=L
$$

This can be expanded into Equation 1.4, where $\phi$ is the phase angle between motion and forcing function, and $\omega=2 \pi f$ is the frequency at which the body is excited. For ease of analysis, the hydrodynamic force in the transverse direction is split into one component in phase with velocity and one in phase with acceleration.

$$
m \ddot{y}+b \dot{y}+k y=F_{0} \sin (\omega t+\phi)=F_{v} \cos (\omega t)+F_{a} \sin (\omega t)
$$

This free vibration system has mass and damping that are dependent on the fluid forces. This enables an effective resonance condition, where the effective natural frequency varies as a function of the fluid forcing. In VIV, these resonant motions occur over a much wider parametric range than in an equivalent mechanical system, because of added inertial forces from vortex shedding and fluid acceleration. 
The specific interplay is shown using the following equations 1.5 and 1.6 of response amplitude and frequency. These can be derived by expanding and nondimensionalizing Equation 1.4 (as shown in several review papers). $m^{*}$ is the mass ratio, $C_{m}$ is the effective added mass coefficient, $f^{*}=f / f_{n}$ is the frequency ratio, $\kappa$ is the transverse force coefficient multiplied by a constant, and $\zeta$ is the damping ratio. The two key features in these equations are the damping and the effective added mass.

$$
\begin{aligned}
& f^{*}=\sqrt{\frac{m^{*}}{m^{*}+C_{m}\left(f^{*}\right)}} \\
& A^{*}=\frac{\kappa \sin \phi}{f^{*}\left(m^{*}+C_{m}\right) \zeta}
\end{aligned}
$$

From equation 1.5, it is seen that as the effective added mass changes, so will the excitation frequency $(f)$. The feedback between the fluid forces on an oscillating bluff body and the resulting body motion can cause the wake-capture phenomenon. This is where the wake frequency $f_{s}$ becomes locked into the structural oscillation frequency $f$. The effective added mass is thereby altered, bringing the natural frequency $f_{n}$ closer to the wake frequency $f_{s}$. Also, as shown in equation 1.6, for low mass-damping $\left(m^{*} \zeta\right)$ values, the amplitude $\left(A^{*}\right)$ is greatly affected by the phase angle $(\phi)$. Even if the magnitude of the forces $(\kappa)$ are low, an appropriate phase angle will make the response amplitude large. This was observed by [99] for low mass-damping cases and is shown in the Griffin plot [142].

\section{Nondimensional Parameters}

There are several parameters that describe the dynamics of this system. Here I pause to define these in their nondimensional form so as to streamline the following discussions. These are then compiled in Table 1.1 .

$\underline{R e}:$

The Reynolds number shows the relative importance of inertia forces compared to viscous forces. The VIV response of cylinders varies somewhat as this value changes [50]. 
$\underline{m^{*} \text { and } m^{*} \zeta:}$

The mass of the cylinder can be normalized with respect to the displaced mass of the fluid to form the mass ratio $\left(m^{*}\right)$. In water, this value is often low since the density of the fluid is on the same order as the density of the material. The damping ratio of the system is written as $\zeta$, which compares the structural damping to the critical damping. The complexity of the VIV phenomena is highlighted in the fact that this damping value is a function of the added mass The effect of these two values are often combined together into a single variable $\left(m^{*} \zeta\right)$. As this value decreases, the amplitude response increases [50].

$\underline{U_{r} \text { and } U_{r n}}$ :

During free-vibration experiments, the flow velocity is varied, which is portrayed through either the true or nominal reduced velocity $\left(U_{r}\right.$ and $\left.U_{r n}\right)$. Similarly, during forced-vibration experiments, the motion frequency $(f)$ and motion amplitude $(A)$ are varied, which affects the reduced frequency $\left(f_{r}\right)$. This is equivalent to the inverse of $U_{r}$.

\section{Others:}

The remaining parameters vary as a function of the reduced velocity: amplitude ratio, frequency ratio, added mass ratio, drag coefficient, and lift coefficient. The analytical solutions to these are derived in the next section. Note that drag (force in the direction of the free stream flow) and lift (force perpendicular to the free stream flow) are written in dimensionless form by dividing by the dynamic pressure: $p_{d}=\frac{1}{2} \rho U^{2} d s$, where $s$ is the cylinder span.

\section{Derivations}

The motion profile of the body can be described with a sinusoidal profile, as can its derivatives:

$$
\begin{aligned}
& y=A \sin (\omega t) \\
& \dot{y}=A \omega \cos (\omega t) \\
& \ddot{y}=-A \omega^{2} \sin (\omega t)
\end{aligned}
$$




\begin{tabular}{|lll|}
\hline name & equation & description \\
\hline reynolds number & $\mathbf{R e}=\frac{\mathbf{U d}}{\nu}$ & inertial force / viscous force \\
\hline mass ratio & $\mathbf{m}^{*}=\frac{\mathbf{m}}{\rho \pi \frac{\mathbf{d}^{2}}{4} \mathbf{s}}$ & cylinder mass / displaced fluid mass \\
\hline damping ratio & $\zeta=\frac{\mathbf{b}}{4 \pi \mathbf{m} \mathbf{f}_{\mathbf{n}}}$ & system damping / critical damping \\
\hline $\begin{array}{l}\text { nominal reduced } \\
\text { velocity }\end{array}$ & $\mathbf{U}_{\mathbf{r n}}=\frac{\mathbf{U}}{\mathbf{f}_{\mathbf{n}} \mathbf{d}}$ & flow velocity / (natural frequency*diameter) \\
\hline $\begin{array}{l}\text { true reduced ve- } \\
\text { locity }\end{array}$ & $\mathbf{U}_{\mathbf{r}}=\frac{\mathbf{U}}{\mathrm{fd}}$ & flow velocity / cylinder velocity \\
\hline reduced frequency & $\mathbf{f}_{\mathbf{r}}=\frac{\mathbf{f d}}{\mathbf{U}}$ & cylinder velocity / flow velocity \\
\hline amplitude ratio & $\mathbf{A}^{*}=\frac{\mathbf{A}}{\mathbf{d}}$ & motion amplitude / diameter \\
\hline frequency ratio & $\mathbf{f}^{*}=\frac{\mathbf{f}}{\mathbf{f}_{\mathbf{n}}}$ & motion frequency / natural frequency \\
\hline $\begin{array}{l}\text { added mass coeffi- } \\
\text { cient }\end{array}$ & $\mathbf{C}_{\mathbf{m}}=\frac{\mathbf{m}_{\mathbf{a}}}{\rho \pi \frac{\mathbf{d}^{2}}{4} \mathbf{s}}$ & effective added mass / displaced fluid mass \\
\hline drag coefficient & $\mathbf{C}_{\mathbf{d}}=\frac{\mathbf{D}}{\frac{1}{2} \rho \mathbf{U}^{2} \mathbf{d s}}$ & $\begin{array}{l}\text { drag force / (stagnation pressure } * \text { projected } \\
\text { area) }\end{array}$ \\
\hline lift coefficient & $\mathbf{C}_{\mathbf{l}}=\frac{\mathbf{L}}{\frac{1}{2} \rho \mathbf{U}^{2} \mathbf{d s}}$ & lift force / (stagnation pressure $*$ projected area) \\
\hline
\end{tabular}

Table 1.1: Nondimensional parameters relevant to VIV. 
The fluid force can also be written using a sinusoidal profile with a phase shift $\phi$ between the force and the motion. This can be expanded in terms of sines and cosines, as in Equation 1.8

$$
\begin{aligned}
F_{y}(t) & =L \sin (\omega t+\phi) \\
& =L \sin (\phi) \cos (\omega t)+L \cos (\phi) \sin (\omega t)
\end{aligned}
$$

The equation of motion (Equation 1.3) can be expanded by plugging in the profiles from Equations 1.7 and 1.8 . Then, two different equations can be written that group terms together based on either having a $\cos (\omega t)$ or a $\sin (\omega t)$ term. These are shown in Equation 1.9 .

$$
\begin{aligned}
b(A \omega \cos (\omega t)) & =L \sin (\phi) \cos (\omega t) \\
\left(m+m_{a}\right)\left(-A \omega^{2}+k A\right)(\sin (\omega t)) & =L \cos (\phi) \sin (\omega t)
\end{aligned}
$$

The amplitude of motion and the fluid forces on the body can be solved by manipulating these equations. The method to do so is simply to nondimensionalize all the terms (using the definitions in Table 1.1) and isolate the desired variable.

Before going through those steps, two more variables should be defined to help simplify the equations. The lift coefficient in phase with velocity $\left(C_{l, v}\right)$ and the lift coefficient in phase with acceleration $\left(C_{l, a}\right)$ are the dimensionless versions of the two components of lift. The lift force can be split into a part that aligns with $\sin (\omega t)$ and one that aligns with $\cos (\omega t)$. There are shown in nondimensional form in Equation 1.10 .

$$
\begin{aligned}
& C_{l, v}=C_{l} \sin (\phi) \\
& C_{l, a}=C_{l} \cos (\phi)
\end{aligned}
$$

Now all the components are in place to solve for the amplitude of motion and the fluid forces on the body. 
$\underline{A / d:}$

Here the steps to do so are shown for the equation that shares the $\cos (\omega t)$ term (Equation 1.9), which leads to the solution of $A / d$ given in Equation 1.11. Again, this is done by nondimensionalizing all terms and isolating the desired variable.

$$
\begin{aligned}
\left(\zeta 4 \pi f_{n} m\right)(A \omega) & =C_{l} \frac{1}{2} \rho d s U^{2} \sin (\phi) \\
\left(\zeta 4 \pi f_{n}\left(m^{*} \rho \pi \frac{d^{2}}{4} s\right)\right)(A(2 \pi f)) & =C_{l, v} \frac{1}{2} \rho d s\left(U_{r n} f_{n} d\right)^{2} \\
\frac{A}{d} & =\frac{C_{l, v} U_{r n}^{2}}{4 \pi^{3}\left(\frac{f}{f_{n}}\right) \zeta m^{*}}
\end{aligned}
$$

$\underline{C_{m}}$

Similarly, the $\sin (\omega t)$ equation can be rearranged into a form that shows the fluid forces on the cylinder, given by the added mass coefficient (Equation 1.12).

$$
C_{m}=\frac{-C_{l, a}}{\frac{1}{4} \pi d^{2} s A \omega^{2}}
$$

$\underline{C_{l, v}:}$

The lift coefficient in phase with velocity is a particularly important variable in this context. It describes the energy transfer between the fluid and the body. The lift force is also assumed to have a sinusoidal shape with a phase lag $(\phi)$. The nondimensional lift is then split into two terms: one in phase with acceleration and one in phase with velocity, as seen in Equation 1.13 .

$$
C_{l}(t)=\frac{L(t)}{p_{d}}=C_{l} \sin (\omega t+\phi)=C_{l, a} \sin (\omega t)+C_{l, v} \cos (\omega t)
$$

The term in phase with acceleration is related to added mass, and the term in phase with velocity is related to damping, or energy transfer. For this reason, $C_{l, v}$ can be used to determine whether energy is being transferred from the fluid to the model (positive sign, which indicates VIV) or vice versa. 


$$
C_{l, v}=\sqrt{\frac{2}{T}} \frac{<C_{l}(t), \dot{y}(t)>}{\sqrt{<\dot{y}(t), \dot{y}(t)>}}
$$

\section{Other Vibrations}

Vortex-induced vibrations fit within the larger class of flow-induced vibrations. In the case of VIV, the vibrations are a result of the large-scale vortex shedding, which causes the motions to be stable and self-limiting (to about $A / d=1$ ) [131]. Another phenomenon within this class include unstable oscillations, such as galloping, which is caused by geometrical asymmetry. These induce large loads, which can lead to catastrophic failures. A third phenomenon is wake-induced vibrations (WIV), where a body vibrates in response to an external vortex wake. In that case, the body can still undergo a lock-in condition with wide bandwidth of response. The vibrations are not self-limiting in that case. This phenomenon will be studied later in the thesis, when the response of the whisker to an externally generated wake is explored.

VIV oscillations are dominantly in the crossflow direction (perpendicular to the free stream). However, there are also oscillations that arise in the in-line direction (parallel to the free stream), which are usually 0.5 diameters or less. These in-line oscillations occur at double the frequency of vortex shedding, since one pair of vortices is shed per cycle on the downstream side of the cylinder.

\subsubsection{VIV Suppression Mechanisms}

The negative impact of VIV on structures, including unsteady forcing as well as drag amplification, has led to the development of multiple means of VIV suppression, as reviewed in [144, 133, 35].

\section{Wake Stabilizers}

One method of VIV reduction is to stabilize the wake by placing items such as fairings, splitter plates, and flags (Figure 1-24(a-c)) into the wake. These streamline the body, which serves to reduce the interaction of the entrainment layers. Two downsides of 


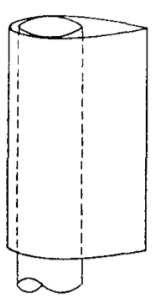

(a)

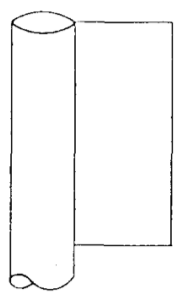

(b)

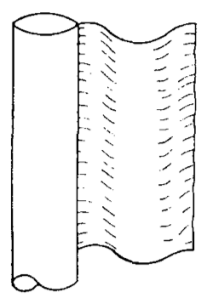

(c)

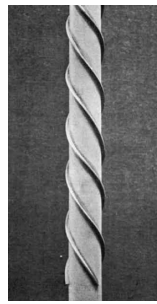

(d)

Figure 1-24: Examples of VIV suppression devices: (a) fairing, (b) splitter plate, (c) flag, and (d) a cylinder with helical strakes. Figures from [35].

these methods are that they require extra material and that their effectiveness is dependent on a unidirectional flow.

\section{Strakes}

Another means of reducing VIV is to change the surface of the cylinder itself. The most widely used suppression device in the offshore oil industry is that of helical strakes (Figure 1-24(d)). These surface protrusions (with height up to $25 \%$ of the diameter) spiral along the length of the cylinder in order to disrupt the coherent formation of vortices.

Such surface modifications can incur the expense of increased drag. In particular, helical strakes experience a higher drag force than a smooth cylinder. This is shown in the log-scale plot of Figure 1-25.

\section{Bumpy surfaces}

Bumps on various objects in nature may serve the purpose of drag reduction [13]. The effectiveness of bumps on a cylinder to reduce VIV was tested [99]. Hemispherical protrusions were attached in a spiral arrangement to a cylinder, as shown in Figure 1-26(a). The wake patterns were seen to differ along the span (Figure 1-26(b)), which allows the drag on the model to be reduced up to $25 \%$ (based on the diameter of the bare cylinder).

It is important to note that as the experiment was changed from fixed boundary conditions to an increasingly flexible mounting (indicated by a lower mass-damping 


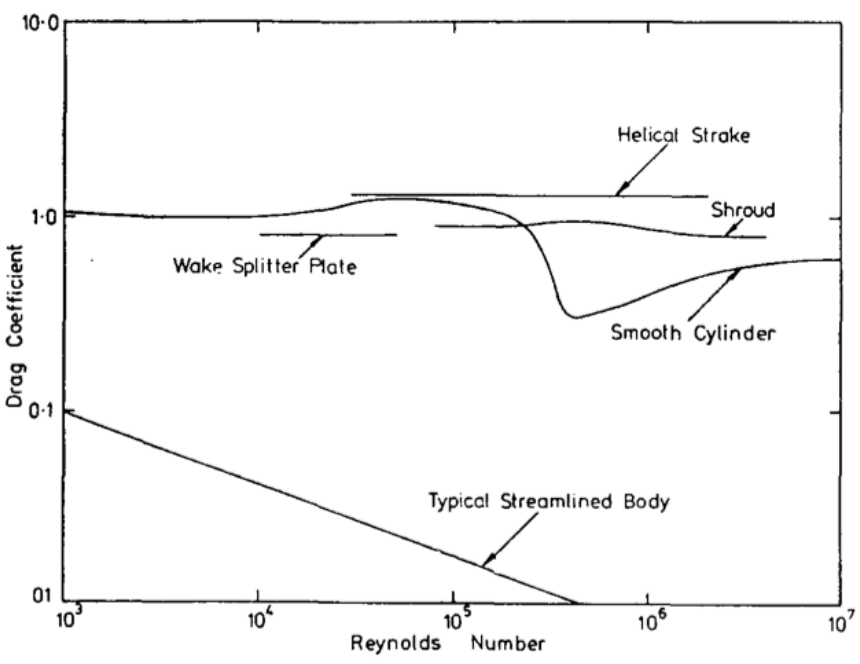

Figure 1-25: The drag coefficient of various VIV suppression devices is higher than that of a smooth cylinder at certain Reynolds numbers. This is especially true for a cylinder with helical strakes. Figure from [35].
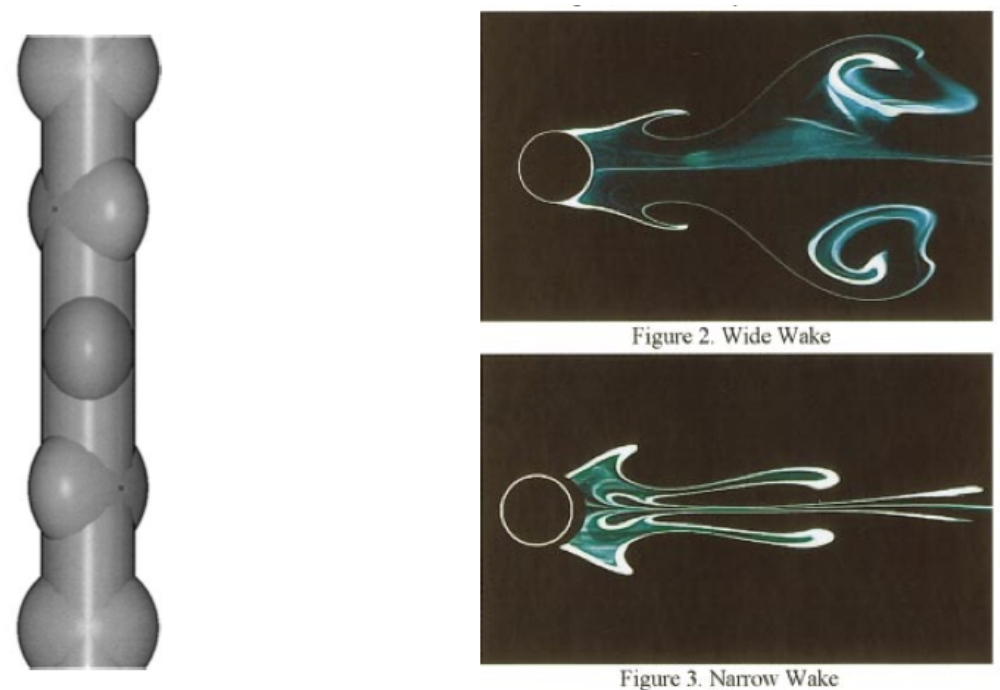

Figure 1-26: (a) A cylinder with bumpy protrusions placed at $45^{\circ}$ angular separation and with pitch of 7 diameters. Figure from [100]. (b) The wake behind this model ranges from wide to narrow, depending on whether the model has a peak or trough in the disturbance there. Figure from [99]. 


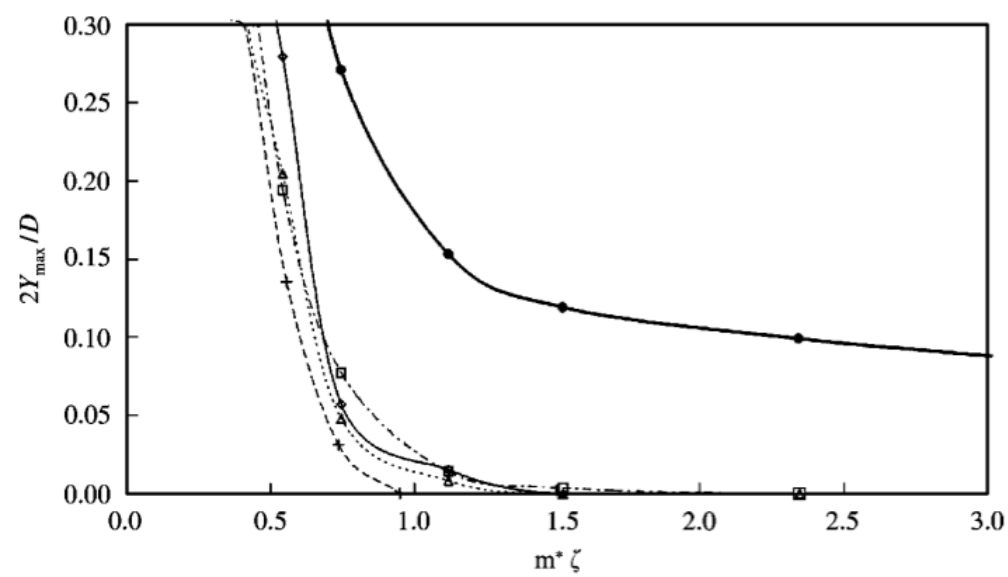

Figure 1-27: Maximum transverse vibration amplitude of different geometries. As the mass-damping $\left(m^{*} \zeta\right)$ decreases, all geometries vibrate with higher amplitude. solid circle: smooth circular cylinder; plus sign: sinuous cylinder; others: bumpy cylinders with various bump sizes). Figure from [99].

value), the transverse response of the model increased substantially. This is the same trend as seen on a smooth cylinder (Figure 1-27), indicating that suppression of vortex shedding under fixed conditions does not necessarily equate to reduction of VIV.

\section{Wavy surfaces}

A final method is seen in the use of wavy surfaces on cylinders. Periodic variations along the span of a cylinder decorrelate vortex shedding. Studies by [2, 75, 81, 80, 83, 145 have shown cylinders of varying waviness effective in reducing the mean drag force by about $20 \%$ and the unsteady lift force significantly, under certain conditions. The effectiveness is attributed to the formation of an undulating wake which decorrelates the induced forces, as well as the formation of streamwise vortices. [83] found a maximum effectiveness when the wavelength to mean diameter ratio, $\lambda / D_{m}$, is close to 2. 80] found a second optimal value of $\lambda / D_{m}=6$, which provides even better performance. The latter value agrees with the reported optimal wavelength to diameter ratio for strakes, which is about 5 for single strakes, and between 15 and 17 for triple start strakes, i.e., effectively, between 5 and 5.6. 

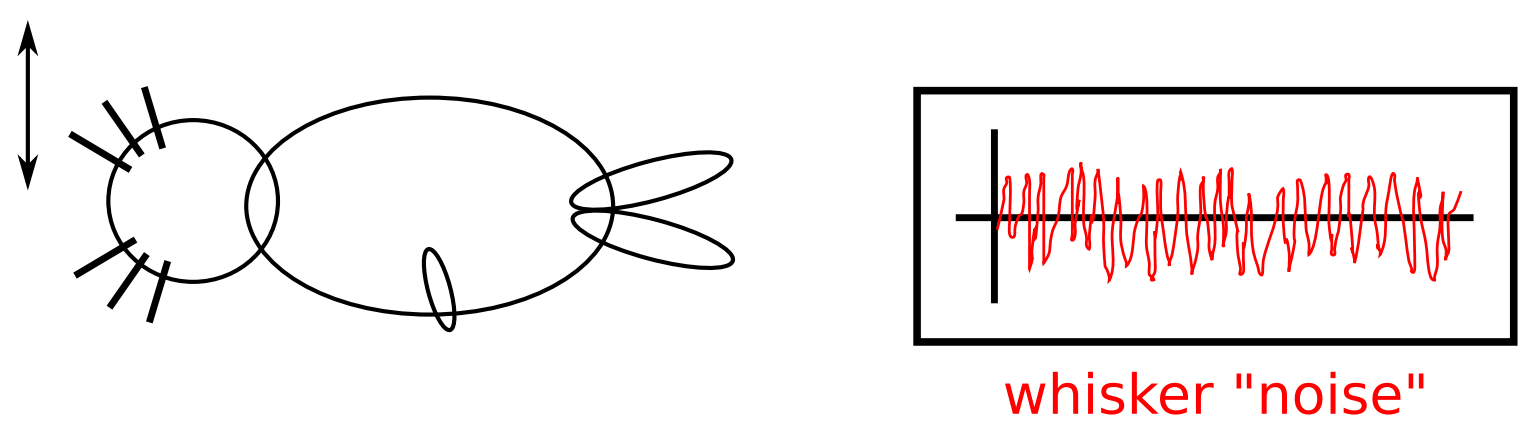

Figure 1-28: Schematic of expected response of the whiskers as the seal is swimming: the whiskers undergo significant VIV, making for a "noisy" signal.

\subsection{Synthesis of Sensing and Vibrations}

These two topics of hydrodynamic perception and flow-induced vibrations are investigated together in the remainder of this thesis.

The reason for their synthesis is triggered by the following (depicted in Figure 1-28): Given that vortex-induced vibrations can result in significant amplitudes of motion on cylindrical objects, we expect that the seals' whiskers should vibrate significantly as the seals are swimming. If the whiskers are sensors, shouldn't they experience strong background noise from VIV?

\subsubsection{Previous Work}

The study by [58] was the first to attempt to synthesize these two topics. The authors made a claim that the whisker geometry was able to suppress VIV. They postulated that this would enable heightened sensing capabilities.

In that study, three tests were conducted. First, a camera was mounted on a harbor seal's head to gain a close view of the whiskers. It was observed that the whiskers did not vibrate (at least beyond the $0.2 \mathrm{~mm}$ resolution of the camera) as the seal swam normally. Second, whisker specimens were placed in a flume. Forces at the base of the undulatory harbor seal whisker were measured to be 6.2 times lower than that of a sea lion whisker, which has a smooth profile. Finally, CFD was conducted on the numerical representation of the harbor seal whisker. The whisker was held rigidly and forces were calculated. Crossflow forces were seen to reduce $90 \%$ and inline forces 

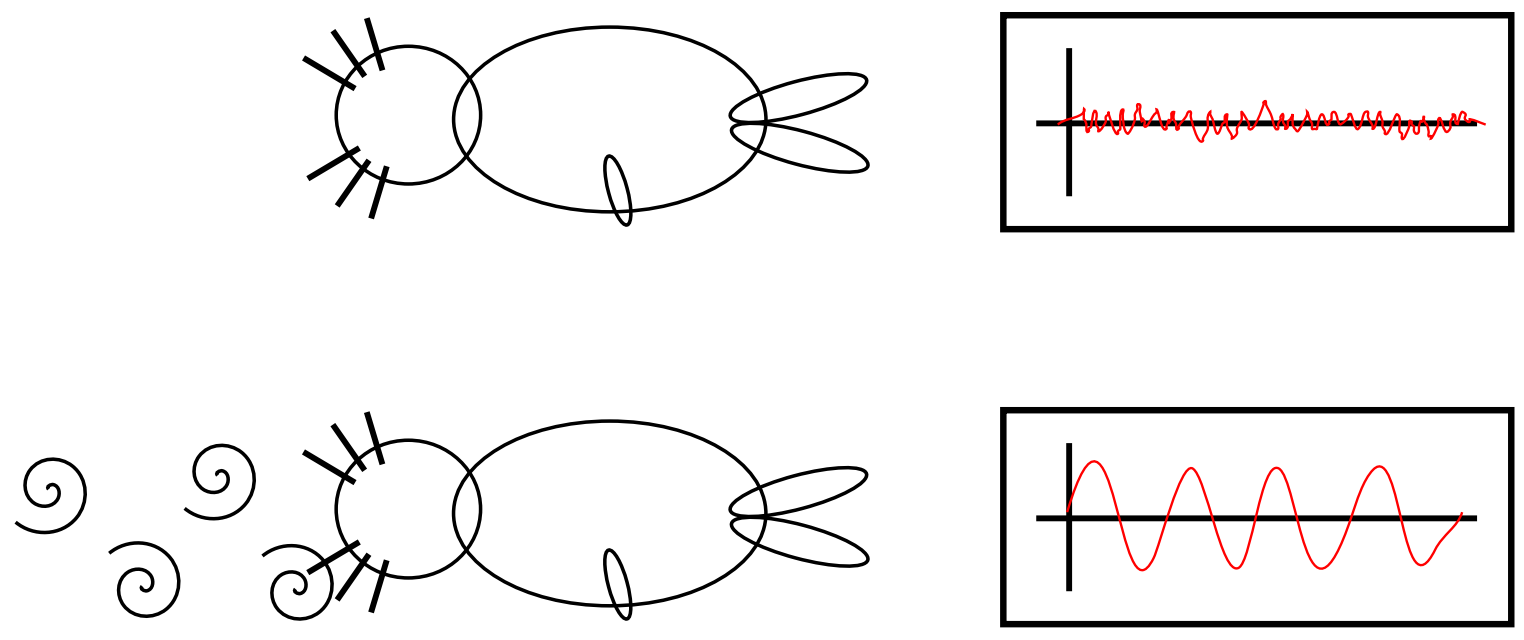

Figure 1-29: Schematic of the hypothesis behind this thesis. In open flow, the whiskers produce low VIV. This enables the seal to more easily detect unsteady flow features, which induce large response.

40\% compared with a smooth circular cylinder.

Their result suggests that the whiskers can move smoothly in a uniform flow, while experiencing few vibrations and therefore accurately measure characteristics of the oncoming flow.

\section{Other whisker vibration studies}

Since then, a few other parties have carried out studies on the vibration properties of seal whiskers on both real and model specimens [91, 143, 95]. The important thing to note is that none of the experiments that provided quantitative results were conducted under conditions that allowed the whisker to vibrate freely in response to the flow. The experiments described in Chapter 2 are designed to address this gap.

\subsubsection{Hypothesis}

The evidence of reduced fluid forcing felt by whiskers in open water [58] leads to the hypothesis of this thesis (depicted in Figure 1-29): The whiskers experience low VIV in open flow so that the seal can more easily detect unsteady flow features. 


\subsection{Outline of Current Study}

This thesis investigates whether or not the harbor seal whisker morphology can, at least in part, be responsible for the heightened sensory ability of the harbor seals. The fluid dynamics of the whiskers' response to different flows is investigated, in order to uncover mechanisms by which vibrational response could aid sensing.

Chapter 1 provided background information on flow sensing in marine animals. Also, the basics of flow-induced vibrations were introduced along with the relevant nomenclature and parameters.

Chapter 2 quantifies the harbor seal whisker geometry's VIV properties. Experiments are conducted using forced vibrations on a scaled whisker model, allowing for dynamic investigation of the vibrational response. Explanations for the whisker's response are provided through wake visualizations and measurement of the added mass and damping properties. The effect of the angle with which the geometry faces the flow is also studied.

Chapter 3 describes the design and characterization of a flow sensor, which incorporates the unique vibration properties discovered in the harbor seal whisker geometry into a free vibration configuration.

Chapter 4 presents experiments of the freely vibrating whisker model encountering a vortex wake. This simple representation of the seal interacting with a fish wake is used to elucidate potential mechanisms employed for wake detection. Strain measurements and dye visualizations reveal the whiskers' unique mode of interaction with external vortices.

Chapter 5 concludes the thesis by summarizing the principal contributions. Suggestions for future work and further hypotheses of the real seals' behavior are provided. 


\title{
Chapter 2
}

\section{Whisker in Open Flow: Forced Vibrations}

\author{
Can harbor seal whiskers passively reduce VIV? \\ How sensitive is the vibration to changes in flow angle?
}

\subsection{Motivation}

In contrast to other animals with whiskers, most phocid seals exhibit a unique whisker geometry consisting of undulating radii in both major and minor axes and an elliptical cross section (average aspect ratio $=2$ ). A close-up view of the whiskers in situ reveals this "beaded" pattern (Figure 2-1). Observations of the behavior of harbor seals suggest that this geometry enables enhanced underwater sensing capabilities. These aquatic animals are known to employ these sensing techniques underwater while swimming with relatively high forward motion, hence the sensing mechanism needs to be sensitive enough to detect minute changes in the local flow field, while robust enough to noise induced through self-excitation from the animal's forward motion.

Few studies have investigated the hydrodynamic interaction of the whisker geometry with its wake and hydrodynamic scaling effects on the geometry, particularly in 


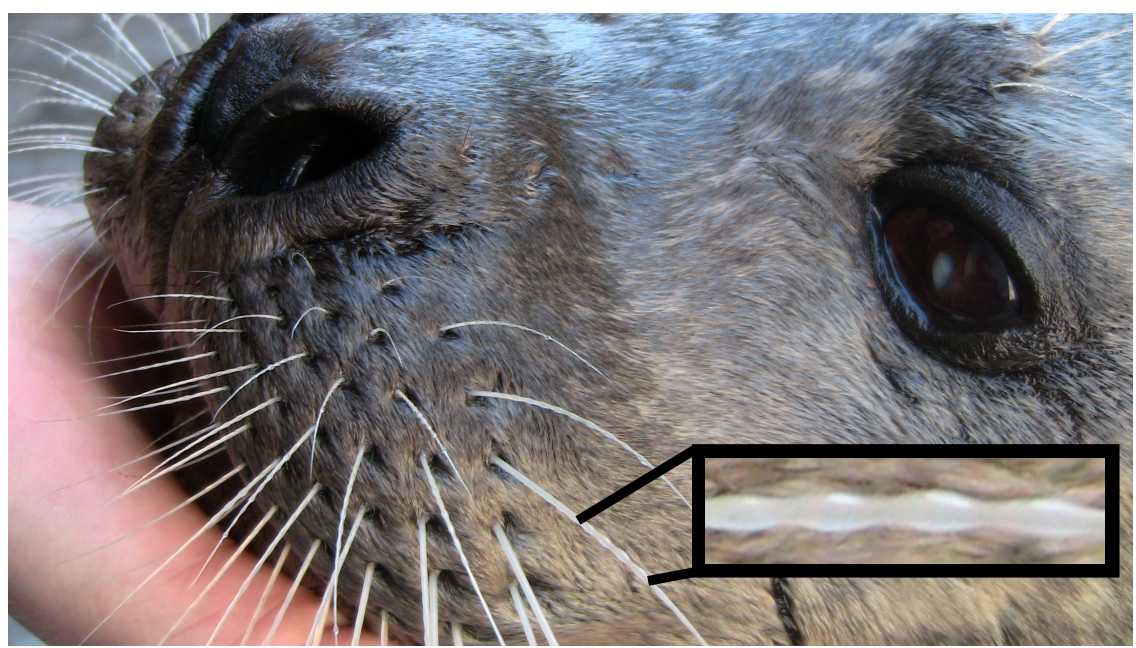

Figure 2-1: Close-up view of the whiskers on a harbor seal's muzzle. The "beaded" pattern indicates an undulatory geometry. Photo taken courtesy of the New England Aquarium.

explaining the effective use of this geometry as a sensor or as a vortex suppression device.

\subsubsection{Whisker in Frontal Flows $\left(\alpha=0^{\circ}\right)$}

[58] posited that the unique geometry of the harbor seal whisker suppresses the natural flow-induced vibrations experienced by the whiskers because of the forward swimming motion of the animal. This statement was based on evidence that vortex shedding is suppressed in the wake of the whiskers when aligned with the direction of swimming for the seal, which is what is referred to here as "frontal flows". This study, however, was limited to a static investigation of the forces exerted on the whisker geometry. For systems near a resonant condition, a reduction in the magnitude of dynamic forces acting on a fixed body does not preclude a reduction in the dynamic response of the body (as seen in [99]). A body's dynamic response is dependent on damping and effective added mass, which influence the phase between the body motion and the fluid forces, potentially leading to lock-in over a wide parametric range.

This chapter examines the full VIV characteristics of the whisker geometry. A scaled whisker model (Figure 2-2) is tested under dynamic conditions and a range of mass-damping values. The goal is to determine if the harbor seal whisker geometry 


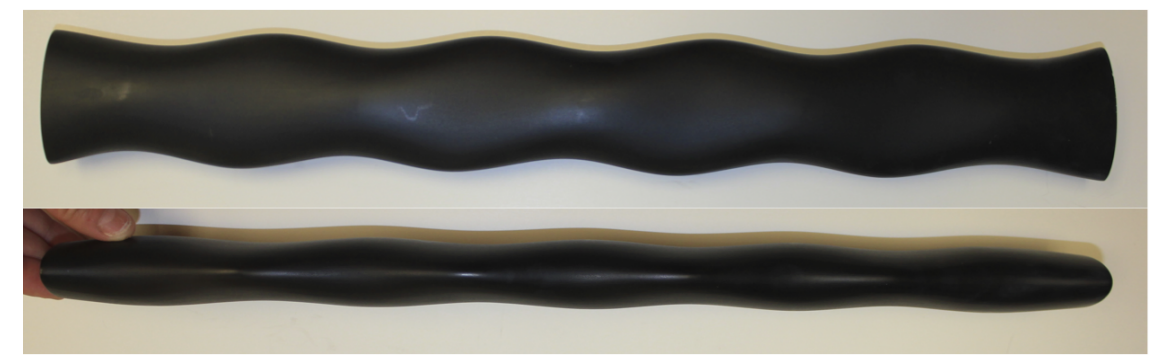

Figure 2-2: (Top) Side view and (Bottom) front view of a fabricated replica of the whisker test section, capturing the asymmetric undulatory geometry. Stereolithography was used to create this plastic hollow structure. Dimensions were taken directly from [58] and enlarged by a factor of 50 .

is able to suppress VIV under conditions that allow for self-excited motions. If it is able, it would imply that the geometry is specialized to reduce the background noise caused by the whiskers own wake in the detection of the upstream target a desirable feature that would motivate a seal whisker-inspired flow sensor.

Experimental force measurements are employed over a range of oscillations to find the parametric regime in which VIV occurs. Flow visualizations are conducted to elucidate the wake patterns associated with these cases. The geometry is first tested in the streamlined direction $\left(\alpha=0^{\circ}\right)$.

\subsubsection{Whisker at Angles of Attack $\left(\alpha>0^{\circ}\right)$}

We lack solid description of the whisker array's position during prey pursuit in the wild, however seals often keep their whiskers erect during tactile sensing [27] and may do so as well during hydrodynamic tracking [18]. This could serve to cover a wide area of the environment. Given that this may happen and that the whiskers may sit in the cheek at different angles within the seals' cheek, it is desired to know what changes the vibrational response may have as the whiskers see the flow from different orientations.

The same methods of experimental force measurement and flow visualizations are used on the whisker geometry undergoing forced vibrations while placed in different orientations to the flow. 


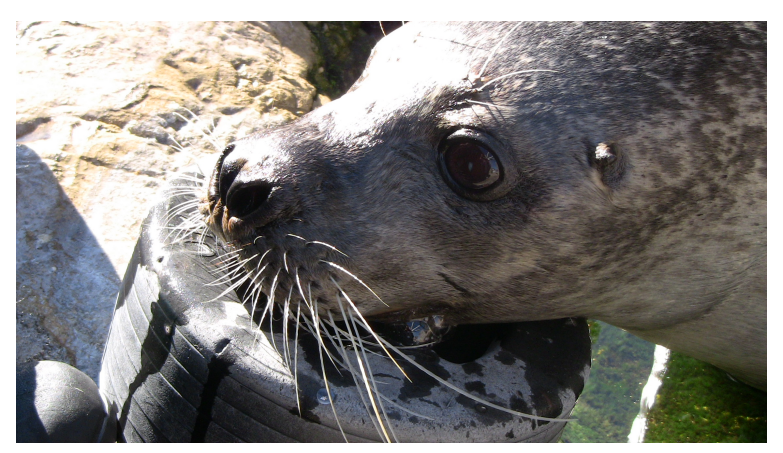

Figure 2-3: Seals's whiskers may face different orientations. Photo taken at the New England Aquarium.

\subsection{Methodologies}

\subsubsection{Force Measurements: Forced Vibrations}

Forced vibration of a cylinder has been shown to accurately predict the fluid forces felt on a freely vibrating cylinder [64, 94, 124, 93]. This is the methodology used to investigate the whisker VIV properties.

\section{Apparatus}

The geometry provided in [58] was scaled up 50 times and fabricated into a model using stereolithography. The model was vertically submerged in a towing tank. It was mounted to a force transducer at its base, which is above the water. A simple harmonic motion profile, given in Equation 2.1, was imposed on the model in the crossflow direction $(y)$ while it was towed (at velocity $U$ ) down the length of the water tank. The expected vibration was thus simulated. Figure 2-4 shows a schematic of this setup.

$$
y(t)=A \sin (\omega t)
$$

The same process was repeated for multiple combinations of amplitudes $(A)$ and frequencies $(f=\omega /(2 \pi))$. These are written in dimensionless form as amplitude ratio $\left(A / d^{*}\right)$ and reduced frequency $\left(f_{r}=f d^{*} / U\right)$. The lift coefficient, where lift is

the hydrodynamic force in the direction of crossflow motion $\left(L=F_{y}\right)$, was recorded 


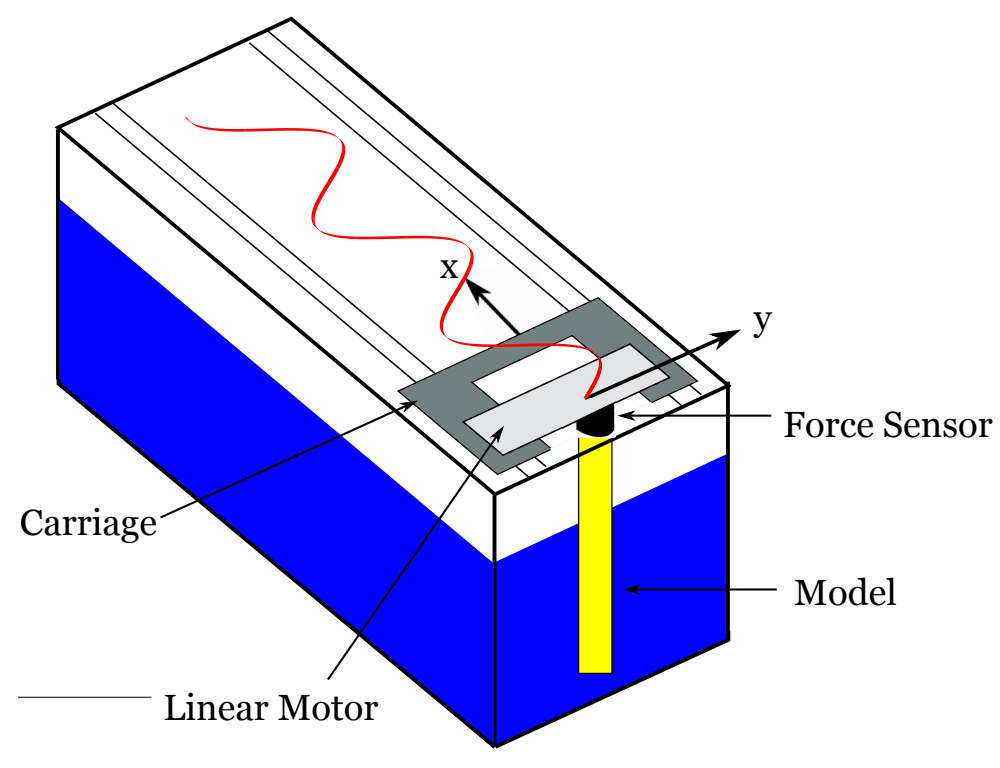

Figure 2-4: Schematic of the forced vibration setup. The model hangs vertically in the water tank. It is directly attached to a force sensor and a linear stage that drives a sinusoidal motion (an example trajectory is depicted in red) while the carriage moves the setup down the length of the tank at velocity $U$.

simultaneously with the position of the model. The phase between these two signals was determined through the calculation of $C_{l, v}$, the lift coefficient in phase with velocity.

The sign of this variable indicates if the model underwent VIV or not. If the value is positive, it indicates an energy transfer from the fluid to the body, which is what occurs during VIV. If the variable is negative, the energy transfer goes from the body to the fluid, so that case is not physical. So those runs that resulted in a value of $C_{l, v} \geq 0$ are cases that could occur if the body was allowed to freely vibrate. For more background on the $C_{l, v}$ variable, refer back to Section 1.2 .2 .

This forced vibration technique is preferable to the traditional free vibration experiment in a few ways. One is the ease of experimental setup. A single linear stage is needed to run the present technique, rather than a full spring-dashpot system that is correctly tuned. Also, with free vibrations, a minor change in flow speed can correspond to a large difference in amplitude and a change in vortex shedding mode. These transitions are challenging to study using free vibration, since the conditions 
cannot be closely controlled. Forced vibrations allow for parameters to be specified with greater precision and to be covered over a wider range. However, the number of experiments required to cover the appropriate parameter space is larger for forced vibrations. There are two variables to be varied (frequency and amplitude), as opposed to one (tow velocity), when using free vibration.

\section{Parameters}

A description of the experimental parameters first start from a reference cross section of the whisker geometry. The reference (Equation 2.2) is taken as an ellipse with inline diameter $(l)$ and crossflow diameter $(d)$ found from the average of the maximum and minimum diameters from the two extreme cross sections. The values shown here correspond to the size of the model used in the first set of experiments (50x-scale model).

$$
\begin{gathered}
d=\left(d_{\text {max }}+d_{\text {min }}\right) / 2=2.65 \mathrm{~cm} \\
l=\left(l_{\text {max }}+l_{\text {min }}\right) / 2=5.35 \mathrm{~cm}
\end{gathered}
$$

The model was tested at different angles of attack $(\alpha)$ with respect to the oncoming flow direction. This angle is depicted in Figure 2-5. First, tests were conducted at $\alpha=0^{\circ}$, such that the orientation is streamlined. Then, different values of $\alpha$ up to $90^{\circ}$ were tested.

The reference diameter used in all cases is the projected diameter $\left(d^{*}\right)$. This is defined as the length of the cross section (the reference cross section given in Equation 2.2 that is perpendicular to the oncoming flow direction. This was calculated for each angle using Equation 2.3. That can be derived by first considering the tangent line to the ellipse that passes through the furthest point of the ellipse on the $\mathrm{x}$-axis, and considering the line perpendicular to that, which also runs through the origin. Then the distance from the origin to the intersection of those two perpendicular lines can be calculated. 


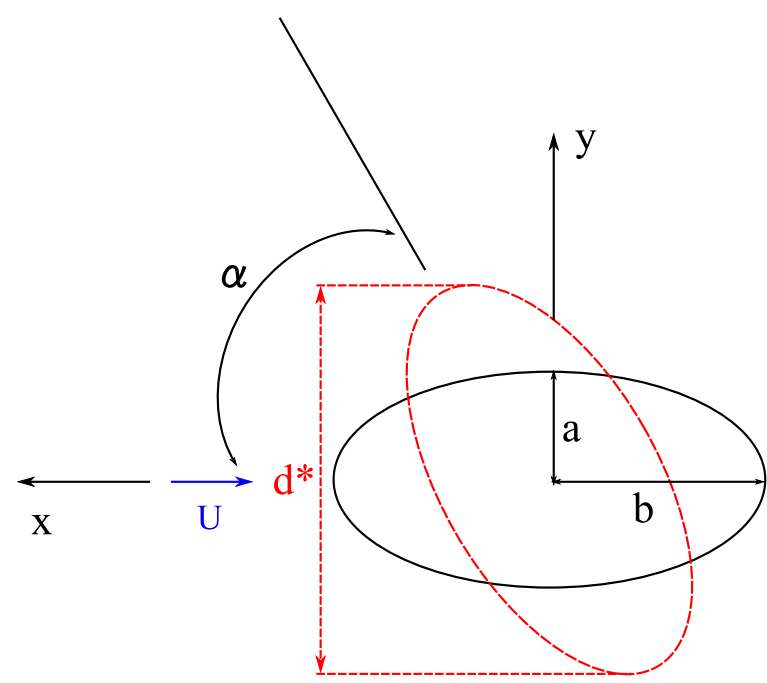

Figure 2-5: Orientation of a cross section of the model. The solid line cross section represents the $\alpha=0^{\circ}$ case and the dotted line cross section represents the $\alpha>0^{\circ}$ cases. The projected diameter $\left(d^{*}\right)$ is the dimension used for all cases and is the length perpendicular to the flow, which approaches with velocity $U$.

$$
d^{*}=2 \sqrt{b^{2} \sin ^{2} \alpha+a^{2} \cos ^{2} \alpha}
$$

Forced vibrations were employed here on three different geometries: the whisker model pictured above (uses the geometry as described in [58]), an elliptical cylinder, and a circular cylinder. All have the same material properties and the same average cross-stream diameter $d=2.65 \mathrm{~cm}$ (at $\alpha=0^{\circ}$ ), which is based on a 50x scale of the real whisker size. For the low angle of attack cases $\left(\alpha<45^{\circ}\right)$, a towing speed of $U=0.2 \mathrm{~m} / \mathrm{s}$ was used, which corresponds to a Reynolds number $\left(R e=U d^{*} / \nu\right)$ of $5,300-7,030$. For the tests at $\alpha>=45^{\circ}$, smaller scale models were used in order to fit the vibration amplitude limits of the experimental setup. This model is a 30xscale version of the real whisker, giving it $d=1.59 \mathrm{~cm}$ (at $\alpha=0^{\circ}$ ). A towing speed of $U=0.1 \mathrm{~m} / \mathrm{s}$ was used, resulting in $R e=2,530-3,210$. The nondimensional length $\left(L / d^{*}\right)$ was the same in all cases, in order to capture the same number of undulation wavelengths. The mass ratio, which is found using the model volume given in Solidworks, is similar for all of the models, ranging from $m^{*}=0.5$ to 1.1 . 


\section{Calculations}

Forces were measured on the models while they underwent imposed transverse $(y)$ oscillations of a certain frequency (written in nondimensional form as reduced frequency: $f_{r}=f d^{*} / U$ ) and amplitude (written in nondimensional form as amplitude ratio: $A / d^{*}$ ) and were towed down the tank at $U=0.2 \mathrm{~m} / \mathrm{s}$. This was repeated for different combinations of frequency and amplitude. These combinations differed for the circular cylinder versus the elliptical cylinder and whisker, as their excitation ranges were found to differ. In the case of the whisker, each of these runs was repeated 10 times and averaged. Signals were acquired with a ATI Gamma 6-axis force sensor (a ATI Nano 6-axis sensor was used for low-amplitude cases on whisker) and collected by a NI USB-6218 DAQ board at a sampling rate of $1000 \mathrm{~Hz}$. The raw data was processed through a two-pole, low-pass Butterworth filter. Inertial forces were calculated by measuring the model's mass and acceleration, and they were removed from the total force signal.

The remaining hydrodynamic force component was used to calculate four variables that describe the vibrational response: the drag coefficient $C_{d}$, the lift coefficient in phase with velocity $C_{L v}$, the effective added mass coefficient $C_{m}$, and the damping ratio $\zeta$.

The drag coefficient was calculated from the in-line force $(D)$ during the forced vibration experiments. It is normalized with respect to the projected diameter $\left(d^{*}\right)$ : Equation 2.4 .

$$
C_{d}=\frac{D}{0.5 \rho U^{2} s d^{*}}
$$

The lift coefficient in phase with velocity, shown in Equation 2.5, depicts the structure's excitation, as the body response amplitude is proportional to the force component that is in phase with velocity (as seen in Equation 1.6). A positive $C_{L v}$ value indicates momentum transfer from the fluid to the body and therefore existence of VIV. Equivalently, a negative value indicates transfer in the opposite direction and no VIV. 


$$
C_{L v}=C_{L} \sin (\phi)
$$

Vortex shedding and acceleration of the surrounding fluid exert forces on the body that are in phase with the body acceleration. Together, these are described by the effective added mass coefficient. The effective added mass is determined in Equation 2.6 by first dividing the lift force in phase with acceleration $\left(L_{a}=L \cos (\phi)\right)$ by the magnitude of acceleration ( $\ddot{y}$ term from Equation 1.4). This is then nondimensionalized with respect to displaced fluid mass in Equation 2.7, where $V$ is volume.

$$
\begin{gathered}
m_{a}=\frac{L_{a}}{\|a\|}=\frac{L_{a}}{-A \omega^{2}} \\
C_{m}=\frac{m_{a}}{\rho V}=\frac{-C_{L, a}}{2 \pi^{3}\left(\frac{A}{d^{*}}\right) f_{r}^{2}}
\end{gathered}
$$

$\zeta$ was found using the same method used to derive $C_{m}$, but now equating the magnitude of the damping term ( $\dot{y}$ term in Equation 1.4 ) to the lift in phase with velocity $\left(L_{v}=L \sin (\phi)\right)$, as in Equation 2.8. The system damping (b) is then nondimensionalized with respect to critical damping $\left(b_{c r}\right)$ in Equation 2.9. Mass ratio $\left(m^{*}\right)$ is used to describe the model mass with respect to the displaced fluid mass.

$$
\begin{gathered}
b=\frac{L_{v}}{\|v\|}=\frac{L_{v}}{A \omega} \\
\zeta=\frac{b}{b_{c r}}=\frac{C_{L, v} V_{r}^{2}}{4 \pi^{3}\left(\frac{A}{d^{*}}\right) m^{*}} \sqrt{\frac{m^{*}}{m^{*}+C_{m}}}
\end{gathered}
$$

\subsubsection{Particle Image Velocimetry (PIV): Steady Flow}

In order to first verify the results shown in [58] for the static case, flow visualizations were conducted using Particle Image Velocimetry (PIV). 


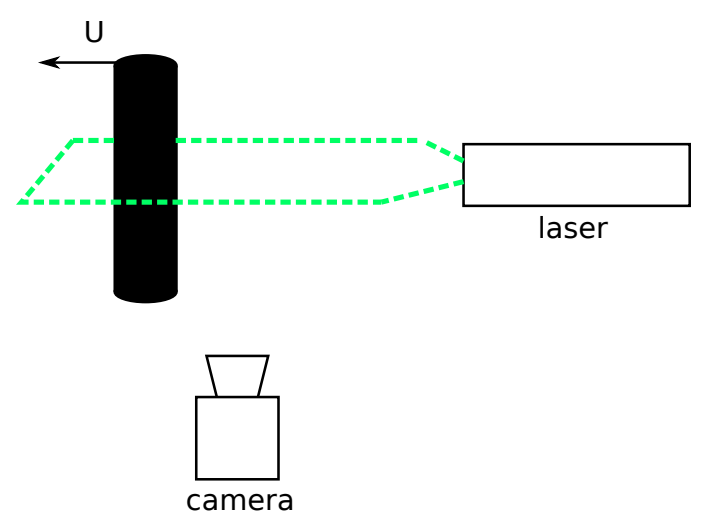

Figure 2-6: Schematic of the PIV setup. The model again hangs vertically in the water tank. A laser sheet illuminates a thin cross-section of the model and the fluid in that plane. A camera mounted below the tank captures the motion of neutrally buoyant particles in the water.

\section{Apparatus}

The model was mounted as before (schematic shown in Figure 2-6), but now in the towing tank at our CENSAM facility in Singapore, and it was towed down the length at $U=0.1 \mathrm{~m} / \mathrm{s}$. A high-speed camera (Phantom V10) operating at 100 frames $/ \mathrm{sec}$ remained stationary below the tank. A continuous wave laser $(\lambda=532 \mathrm{~nm})$ located behind the model illuminated the particle-laden flow with a horizontal sheet of light at $40 \%$ span. Cross-correlation of the captured images was used to determine the motion of the particles within a 128x128-pixel sized interrogation window followed by a $64 \times 64$-sized window, both with $50 \%$ overlap. Light smoothing was carried out through use of a $3 \times 3$ filter. The same thing was repeated for an elliptical cylinder of the same average diameter as the whisker in both directions (2:1 ratio).

\subsubsection{Particle Image Velocimetry (PIV): Forced Vibrations}

The same setup and processing steps used in the steady flow PIV experiments was used again, but now with imposed motion profiles to view the wake under dynamic conditions. Based on the force measurements, the runs corresponding to the maximum VIV amplitude were chosen and used for visualization here. For the whisker model, this corresponds to $A / d^{*}=0.075, f d^{*} / U=0.33$, and for the elliptical cylinder, this corresponds to $A / d^{*}=0.175, f d^{*} / U=0.27$. 


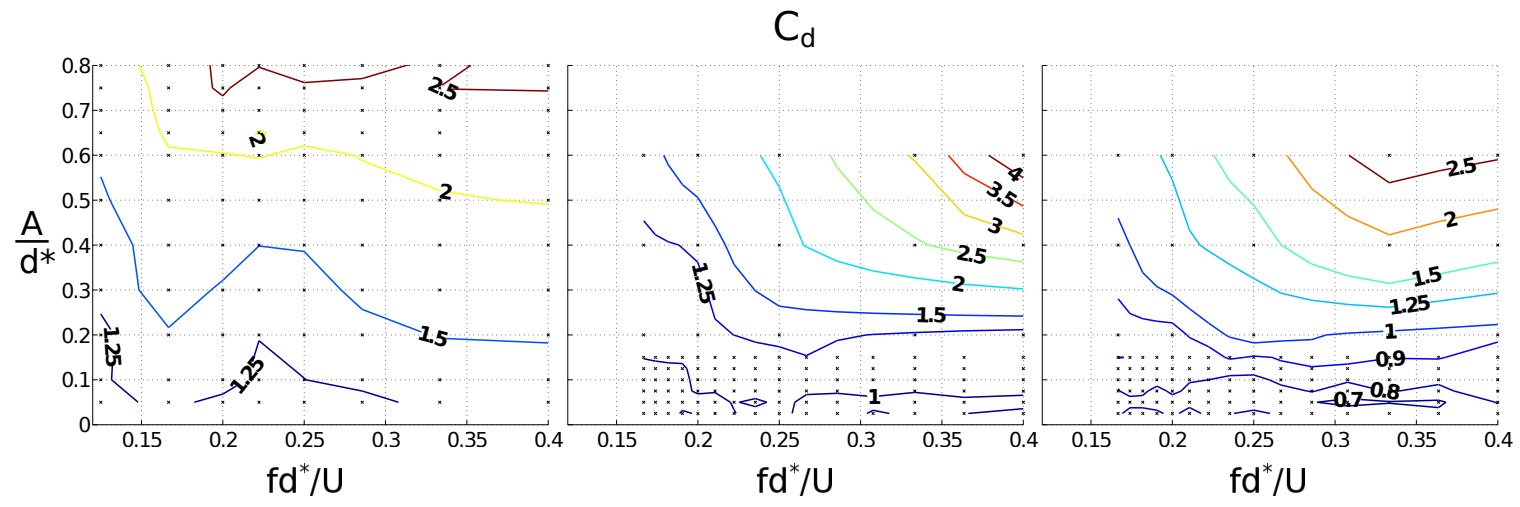

Figure 2-7: $C_{d}$ is plotted as a function of amplitude ratio and reduced frequency for the circular cylinder, elliptical cylinder, and whisker (from left to right). $R e=$ 5,300. Asterisks indicate where experiments were performed. The contour levels are in increments of 0.1 up to 1 , then in increments of 0.25 to 1.5, and in increments of 0.5 beyond that.

\subsection{Results $\left(\alpha=0^{\circ}\right)$}

\subsubsection{Force Measurements}

\section{$\operatorname{Drag}\left(C_{d}\right)$}

Figure 2-7 shows a comparison of the models' responses during the forced vibration tests. The drag coefficient goes from 1.25 to 1 to 0.7 for the circular cylinder, elliptical cylinder, and whisker in the near-zero amplitude case. This shows that the whisker geometry reduces the mean drag one of the two desired components for VIV suppression.

The drag is defined as the hydrodynamic force acting on the body in the in-line direction $(D)$. The formula was given in nondimensional form in Equation 2.4, where $s$ is the submerged span.

If one compares the drag during a forced vibration (crossflow) of the same reduced frequency and amplitude ratio (for example, $f_{r}=0.2$ and $A / d^{*}=0.4$ ), the drag is seen to decrease from circular cylinder to elliptical cylinder to whisker. In that example, it goes from 1.9 to 1.3 to 1.1. Comparison of drag during zero vibration gives values of 1.5 to 1 to 0.8 , in the same order. 


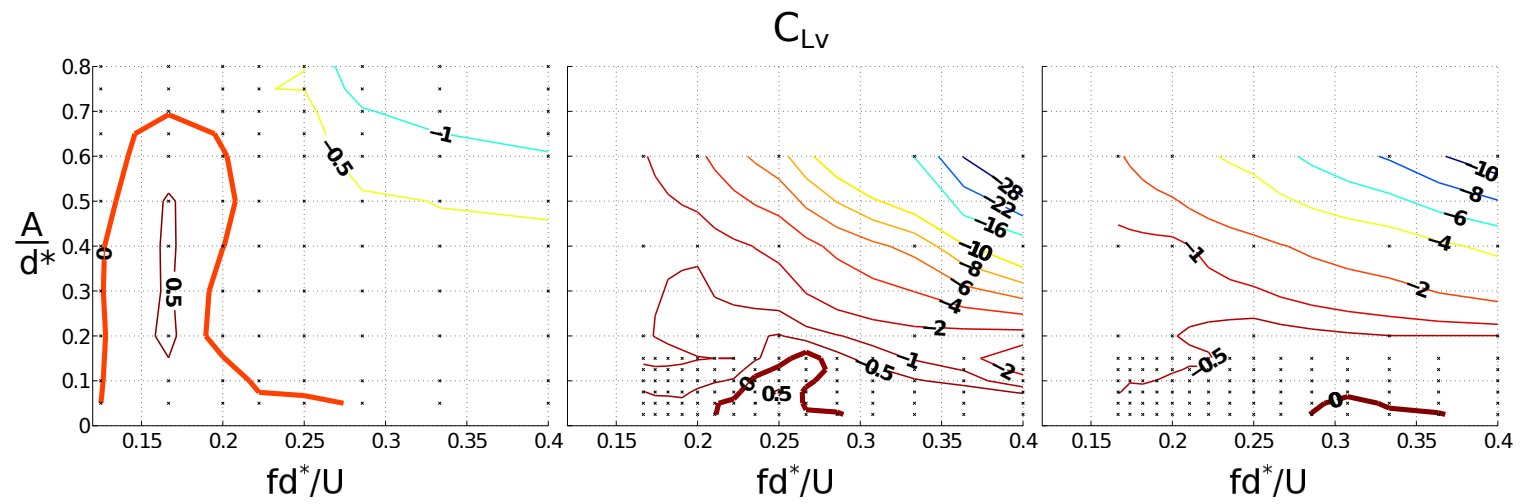

Figure 2-8: $C_{L v}$ is plotted as a function of amplitude ratio and reduced frequency. The region of positive values indicates where VIV occurs. The size of this region decreases from the circular cylinder to the elliptical cylinder to the whisker (from left to right). The zero contour is depicted in bold. $R e=5,300$. Asterisks indicate where experiments were performed. The contour levels are in increments of 0.5 down to -0.5 , then in increments of 2 from -2 on, and in increments of -6 beyond that.

\section{Energy Transfer $\left(C_{l, v}\right)$}

The second desired component is the reduction in vibration response (amplitude and bandwidth). This is calculated via $C_{L v}$, which is plotted in contours against amplitude ratio $\left(A / d^{*}\right)$ and reduced frequency $\left(f_{r}\right)$ in Figure $2-8$. Presented in this manner, the full VIV characteristics, including potential self-excited motions, are captured. The asterisks indicate the frequency and amplitude values of the multiple runs conducted. In the whisker case, the $C_{L v}$ values displayed are the average of 10 trials for each asterisk. The area of positive value (the zero contour peaks at $A / d^{*} \approx 0.075$ ) for the whisker is drastically smaller than that of a circular cylinder. Thus, the regime in which VIV is experienced by the whisker is largely suppressed. The baseline case of a cylinder with the same cross-stream diameter has peak vibration at $A / d^{*} \approx 0.7$, which aligns well with the value found by [49]. The elliptical cross section plays a significant role in reducing the vibrations, since the elliptical cylinder exhibits a peak vibration of $A / d^{*} \approx 0.18$.

\section{Variation in $C_{L, v}$}

Another interesting point is that there is variance in certain $C_{L v}$ values across the 10 trials. While some runs appear with negative $C_{L v}$ on average, they still experience 
a positive value some of the time. The probability that those runs will experience VIV based on the ten trials, is shown in Figure 2-9 as a third dimension on top of the average contour plot. It is seen that values of $C_{L v}$ fluctuate in time, so while they may show up negative on average, the actual value is positive once lock-in has been reached. The whisker, therefore, is shown to require a longer time to reach lock-in than a cylinder. The length of tank used limited the length of time available to capture data. This variability also suggests that a greater variation in shedding pattern along the whisker span versus the cylinder.

An explanation for this variation can be unveiled if we look at the $C_{L, v}$ value versus time. The value actually fluctuates over time (refer to subplots in Figure 2-9). For these low-amplitude cases, lock-in is not reached until multiple cycles of oscillation have occurred. The scatter plots shown for each cycle depict the spread of $C_{L, v}$ values over 10 trials. The middle black line with dots shows the ensemble average for each cycle. With the exception of Run $c$, these cases have a decreasing standard deviation of $C_{L, v}$ over time, and these values eventually settle into a region that is consistently either positive or negative. Run $c$ does not appear to have settled into a constant value, but the sign is not in question it is clearly positive after the initial phase.

This extended time needed for the whisker's wake to synchronize (compared to a cylinder) means that only the latter portion of data should be considered in the analysis. The initial start-up phase can be discarded from the calculation of average $C_{L, v}$. It is chosen here to only consider the segment of data where the standard deviation has reached half its initial value. Using that shortened data set, the average of the ensemble averages is then calculated and is stated as the representative $C_{L, v}$ value for that point on the plot. This means that Runs $b, c, e$, and $f$ can be considered always positive and Run $a$ can be considered negative. Although Run $c$ does not have enough time to settle in, it clearly reaches a positive value. 


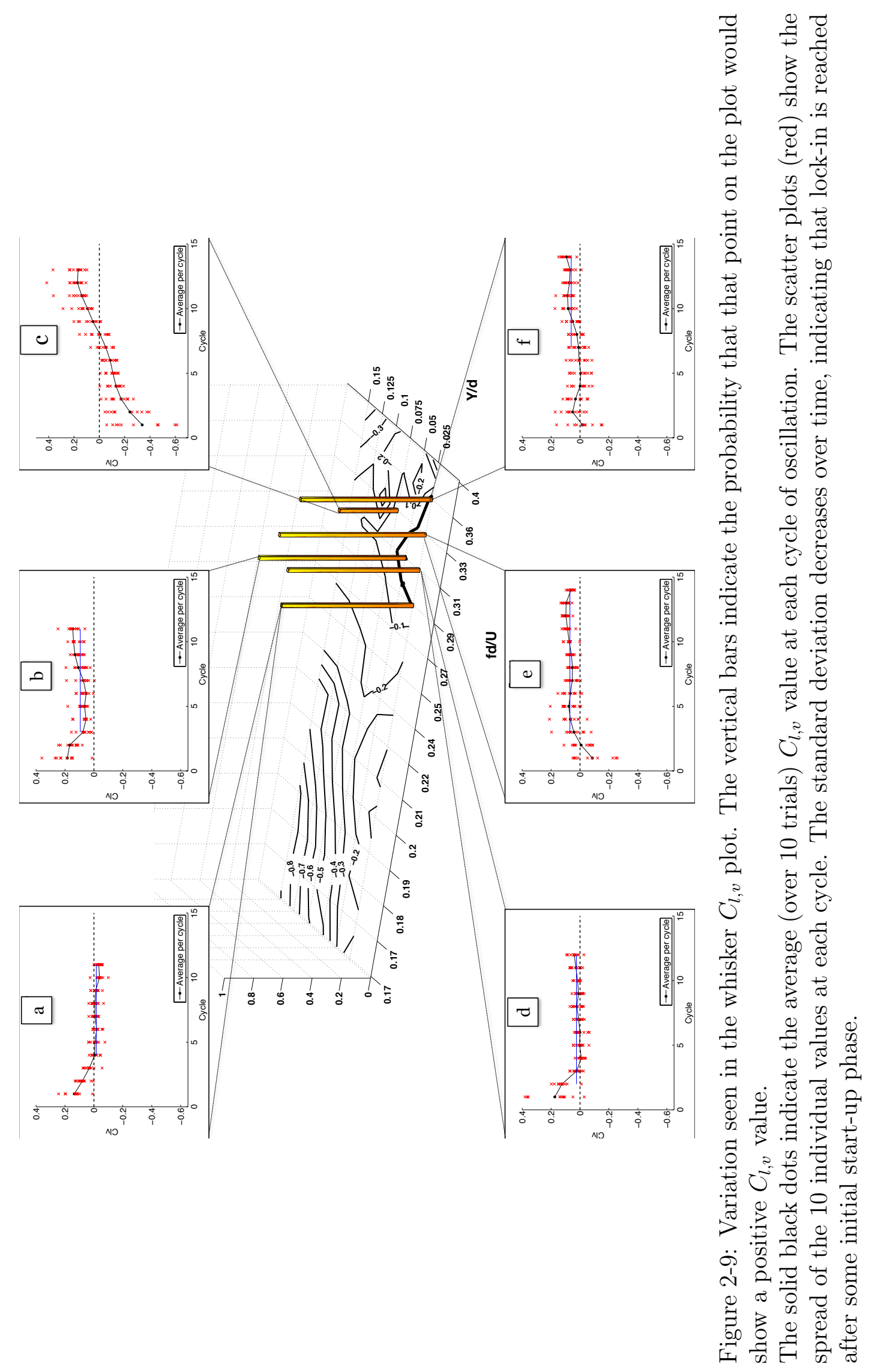




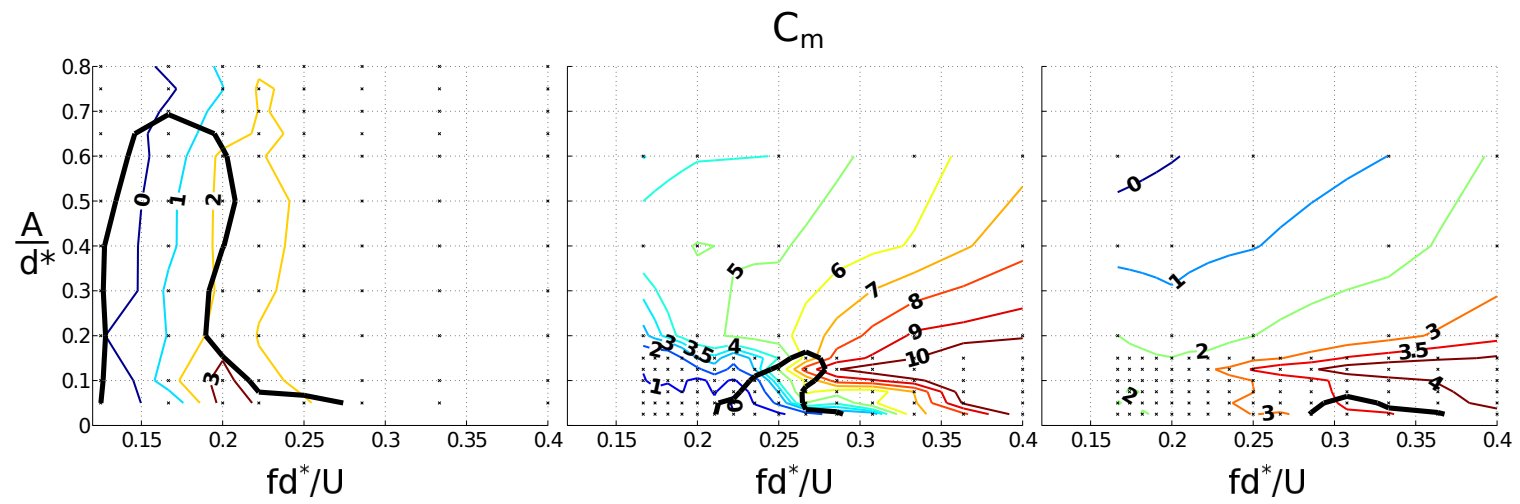

Figure 2-10: $C_{m}$ contours plotted as a function of amplitude ratio and reduced frequency for the circular cylinder, elliptical cylinder, and whisker (from left to right). The zero $C_{L v}$ contour is copied over and shown in black. The contour levels are in increments of 1 and the 3.5 contour is included as well. Asterisks indicate where experiments were performed.

\section{Effective Added Mass $\left(C_{m}\right)$}

Figure 2-10 shows the effective added mass coefficient for the circular cylinder to range from slightly negative to 3 within the excitation region (as denoted by the zero contour of $C_{L, v}$ ), which matches the expected values in literature [49]. Those values deviate from the potential flow solution of 1 , which means that the wake forces the body into lock-in over a wide range of frequencies. The elliptical cylinder has a potential flow added mass value of 4 , but sees values ranging from 0 to 9 . It reaches an even larger range ( 0 to 9 ) because of the doubly sized dimension that moves in the cross flow direction. So in this case, the wake has a particularly strong effect on the VIV response. The shed vortices are coherent and therefore force lock-in over a range of frequencies, despite the reduced amplitude of response. The whisker, however, sees a small $C_{m}$ range: 3.3 to 3.6 , indicating that the wake plays a much smaller role in causing lock-in. The vortices are less coherent, so the wake can not easily force the model to lock-in. This indicates that the whisker is able to reduce the bandwidth of response. Also, this shows that the added mass is about 15\% larger for the whisker than the cylinder, giving a partial explanation for why the whisker geometry is so effective at reducing vibrations. 

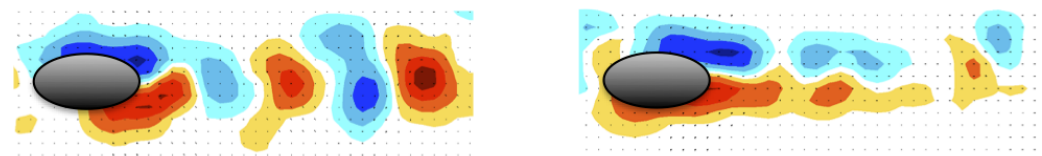

Figure 2-11: Vorticity contours of a 2-D slice of the wake behind the elliptical cylinder (left) and whisker (right) models undergoing steady tow. A standard von Kármán vortex street appears behind the ellipse, but the whisker decorrelates the vortex shedding, causing the vortex street to appear much farther downstream, where the forcing on the body is reduced.

\section{Damping $(\zeta)$}

The circular cylinder sees values of 0 to 0.4 in the excitation region, the elliptical cylinder reaches 1.6, and the whisker goes to 0.1 . These values also illustrate the difference between the elliptical cylinder and the whisker. Although the elliptical cylinder reduces the amplitude of vibration, lock-in is still guaranteed to happen even if the damping reaches the high value of 1.6 at low amplitudes. The whisker on the other hand, only guarantees lock-in if the system damping is less than 0.1 at low amplitudes.

\subsubsection{PIV}

\section{Steady Flow}

First, the wakes behind the whisker and elliptical cylinder undergoing towing motion with no vibrations are observed using PIV. These wakes are compared at a single point in time in Figure 2-11. The whisker wake presents a von Kármán street farther downstream than the ellipse. Also, the vortices in the whisker wake are less coherent. These indicate reduced forcing on the whisker and agree with the conclusion presented in [58]. The flow patterns match well with those shown behind a bumpy cylinder by [99]. 

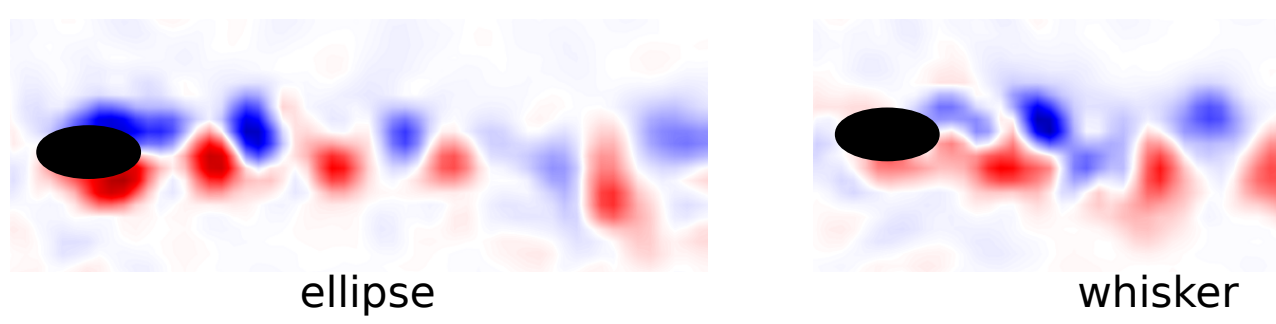

Figure 2-12: Vorticity contours of a 2-D slice of the wake behind the elliptical cylinder (left) and whisker (right) models undergoing the forced vibration run corresponding to their maximum VIV amplitude. A standard von Kármán vortex street appears behind both models.

\section{Forced Vibrations}

The wakes behind both models are shown in Figure 2-12 under conditions corresponding to each model's respective maximum VIV amplitude (elliptical cylinder: $A / d^{*}=0.175, f d^{*} / U=0.27$, whisker: $\left.A / d^{*}=0.075, f d^{*} / U=0.33\right)$. In these cases, both models form a von Kármán vortex street. As in the steady case, the whisker's wake is less coherent than that of the elliptical cylinder. The difference, however, in coherence and formation length is less dramatic, highlighting the importance of including motions.

\subsection{Results $\left(\alpha>0^{\circ}\right)$}

The same method of forced vibrations was conducted on the whisker and elliptical cylinder models, while facing the flow at an angle $\left(\alpha>0^{\circ}\right)$. Experiments were conducted in increments of $15^{\circ}$ up through $90^{\circ}$. Due to size limitations in the experimental setup, smaller models were used for the tests at $\alpha \geq 45^{\circ}$.

\subsubsection{Force Measurements}

\section{$\operatorname{Drag}\left(C_{d}\right)$}

The drag on the whisker at different angles of attack is shown in Figure 2-13. During a zero-amplitude vibration case, the drag coefficient is approximately $1.2,1.5$, and 2 for the 15,30 , and $45^{\circ}$ cases, respectively. The 60,75 , and $90^{\circ}$ cases are about 2.5 . 
The elliptical cylinder responds similarly for the high angle cases ( $45^{\circ}$ and up).

Both models respond similarly to each other, which is at values higher than that of the cylinder. The benefit of using the whisker geometry to reduce drag is therefore lost when the whisker does not face the flow in the streamlined position.

\section{Energy Transfer $\left(C_{l, v}\right)$}

$C_{l, v}$ measurements shown in Figure 2-14 reveal that vibrations in both the whisker and elliptical cylinder increase with angle of attack. The whisker's amplitude at $\alpha=0^{\circ}$ is about half of the elliptical cylinder's amplitude. Up through $\alpha=30^{\circ}$, the two models continue to respond with low vibration amplitude $\left(A / d^{*} \approx 0.2\right.$ or less). About $45^{\circ}$, the amplitude ratio jumps up and continues to increase through $\alpha=90^{\circ}$, where both models respond with large amplitude $\left(A / d^{*} \approx 1.5\right)$. This value is about twice as high as that experienced by a circular cylinder (from Figure 2-8; $\left.A / d^{*} \approx 0.7\right)$. The whisker's undulations are too small relative to the bluff dimension to aid desynchronization of vortex shedding at high angles of attack. Figure 2-15 displays this trend in $15^{\circ}$ increments.

Use of the whisker geometry as a vibration suppression device in scenarios where flow approaches the body from a range of directions is therefore unsuitable. However, the wide spectrum of vibrational response (factor of $\sim 20$ ) may be advantageous for the seal and can be exploited in an engineering sensor.

\section{Effective Added Mass $\left(C_{m}\right)$}

The effective added mass coefficient $C_{m}$ of the whisker and ellipse at different angles of attack is shown in Figure 2-16. The results indicate that the models experience VIV over a wide range of response. At $90^{\circ}$, the whisker and elliptical cylinder have $C_{m}$ values of $\sim 0$ to -2 . This relatively large deviation from the potential flow value of 1 shows that the wake adjusts to encourage VIV. 


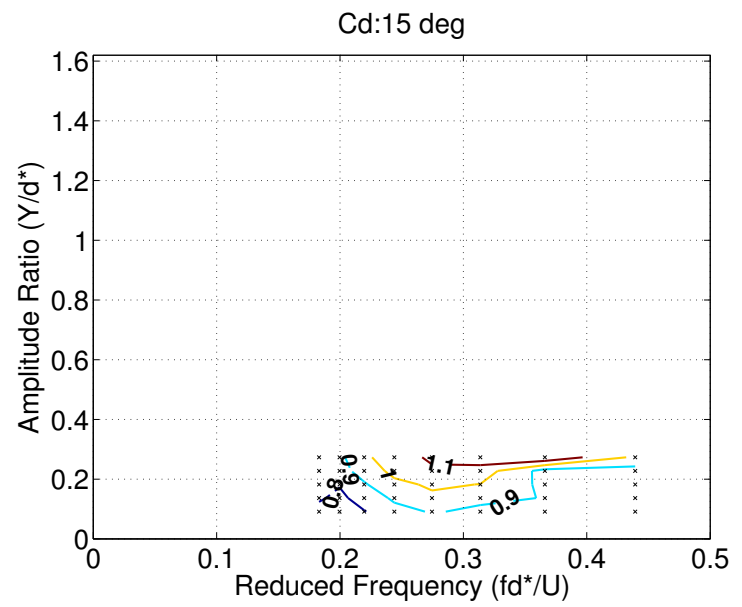

(a)

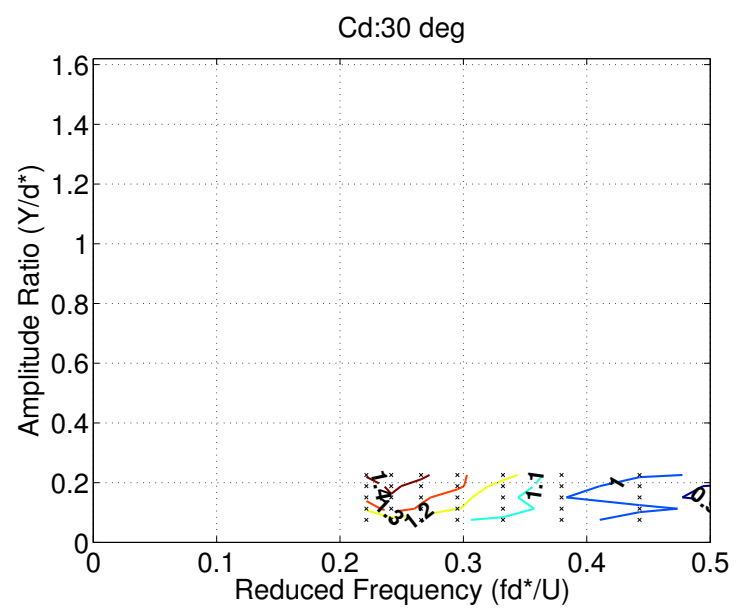

(c)

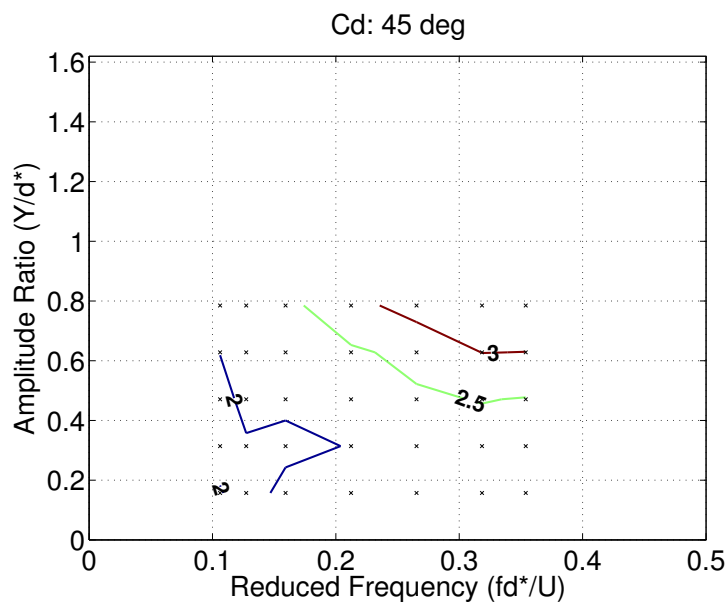

(e)

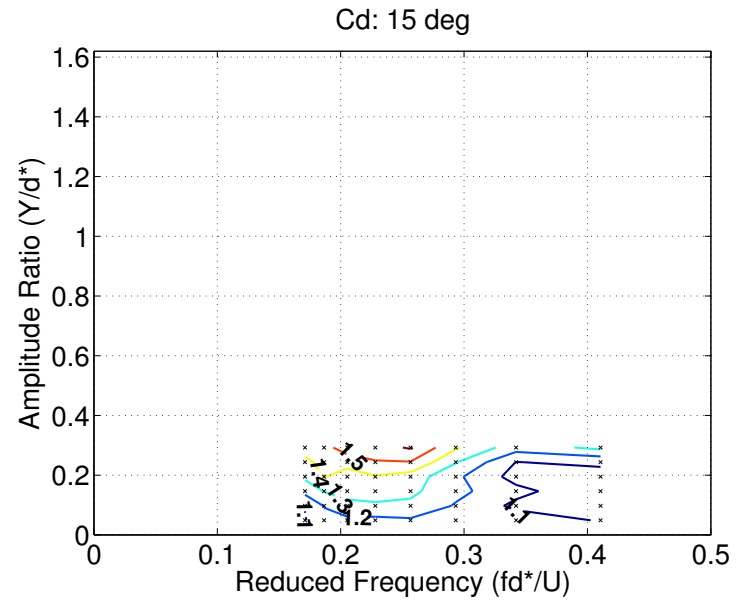

(b)

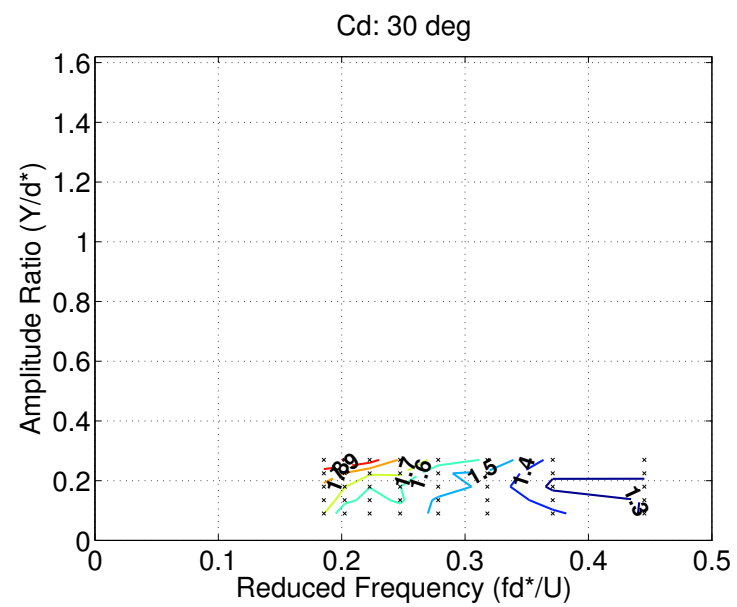

(d)

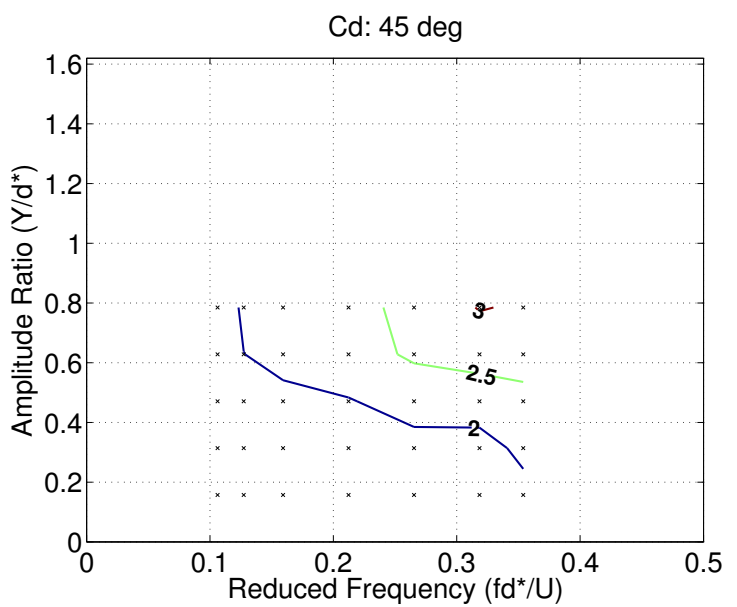

(f) 


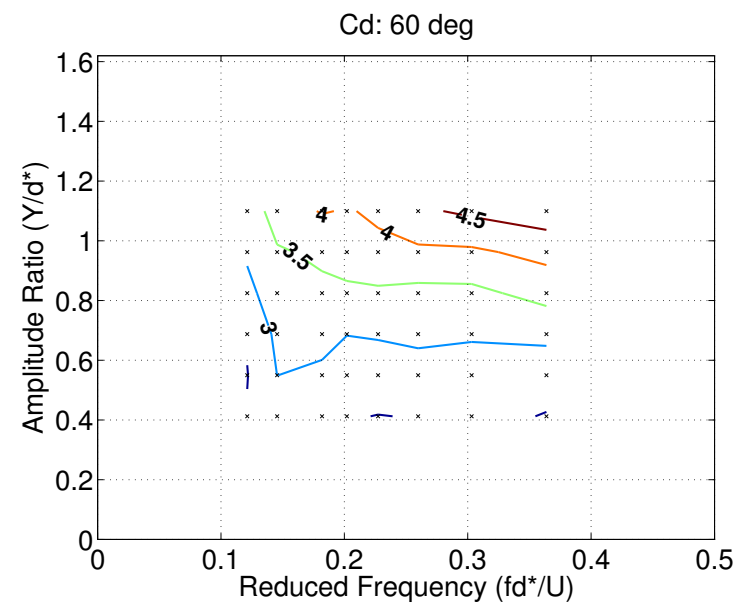

(a)

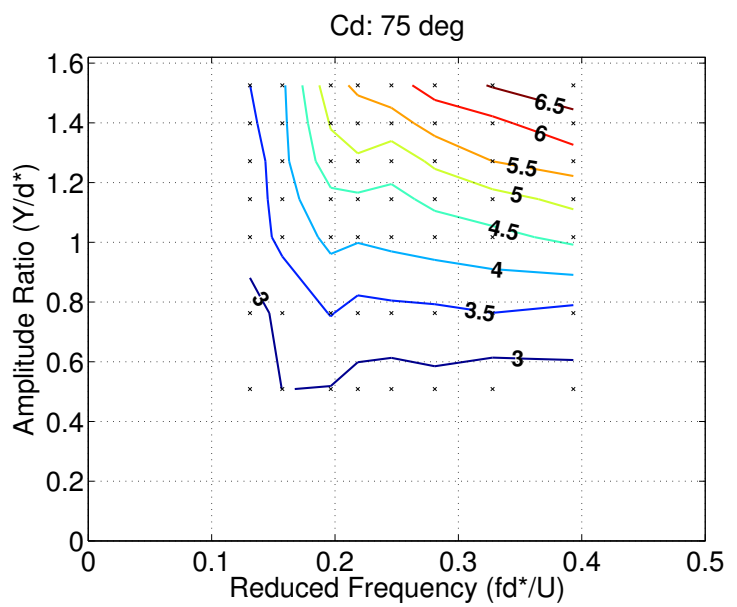

(c)

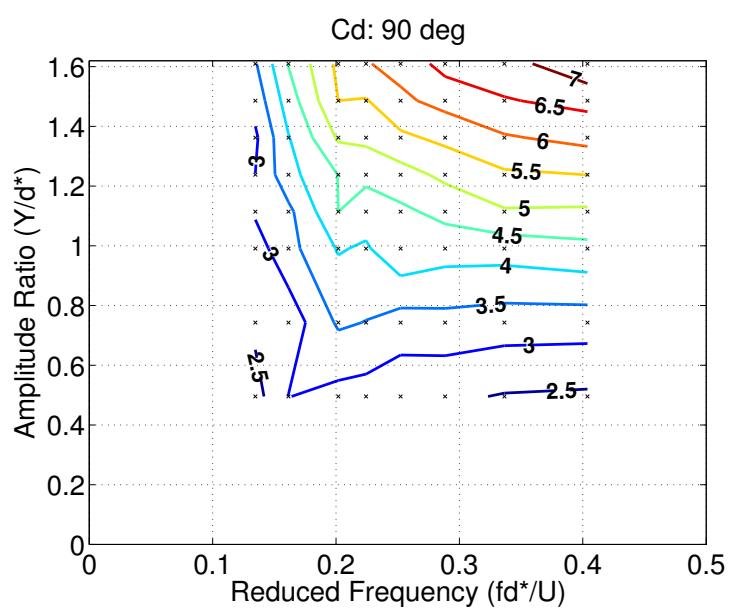

(e)

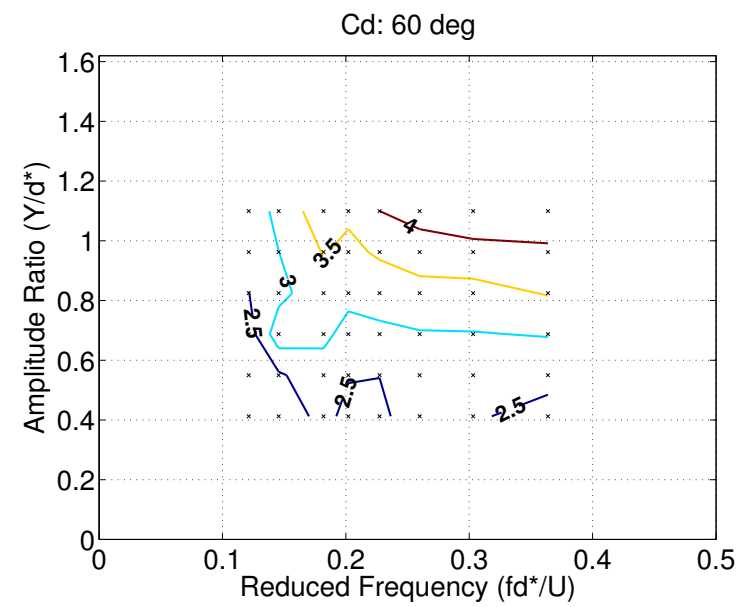

(b)

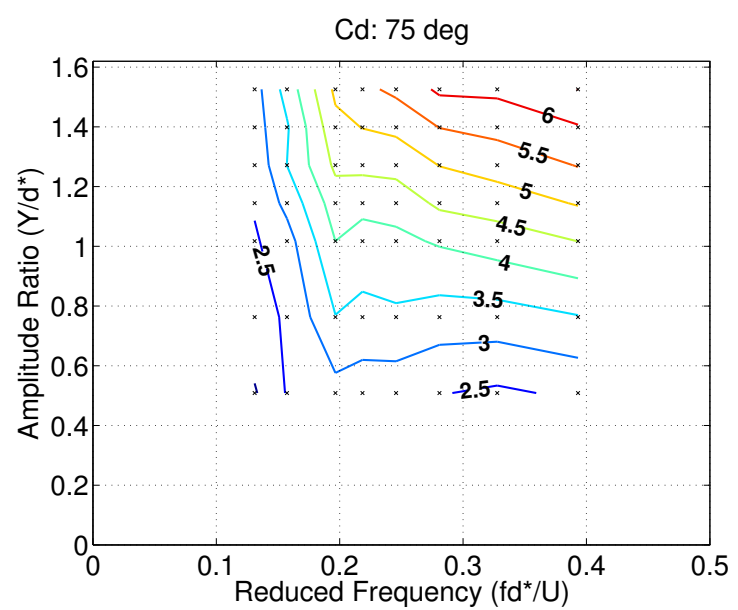

(d)

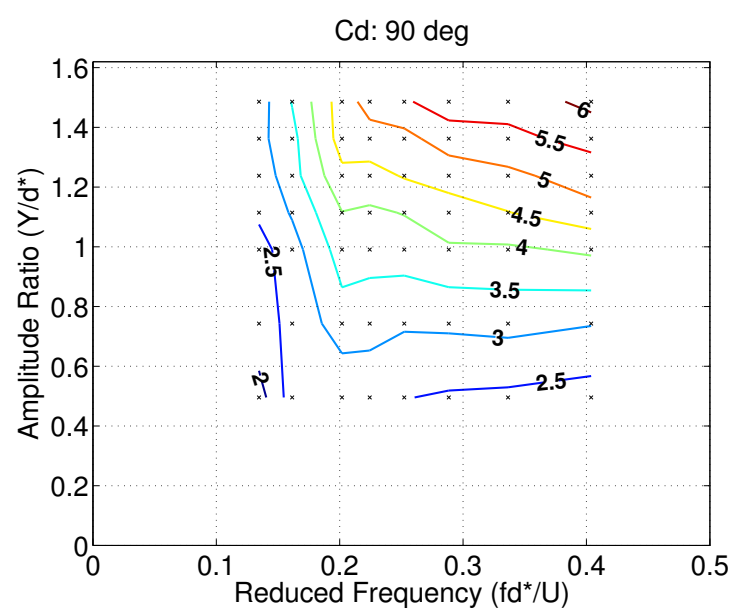

(f) Whisker 90

Figure 2-13: Drag coefficient during forced vibrations for (left column) ellipse and (right column) whisker at (a,b) 15; (c,d) 30; (e,f) 45; (g,h) 60; (i,j) 75; (k,l) 90. Contour levels for 15 and $30^{\circ}$ are in increments of 0.1 . Otherwise they are in increments of 0.5 . 


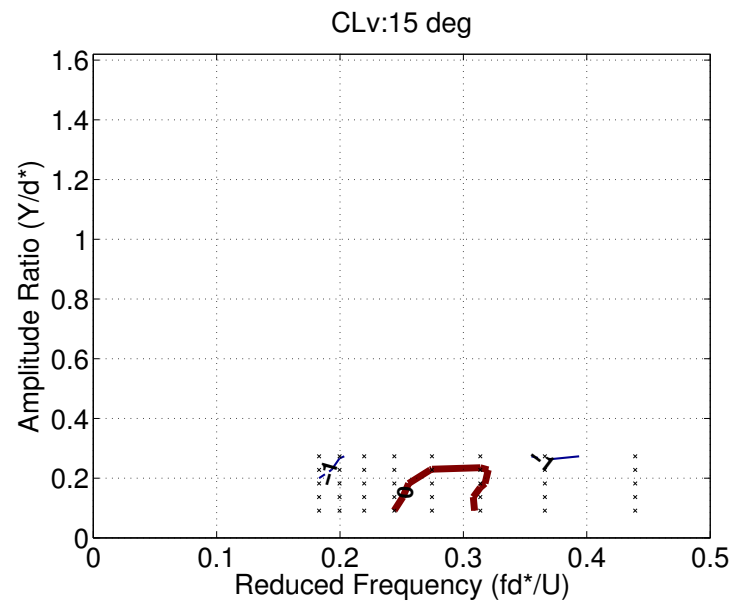

(a)

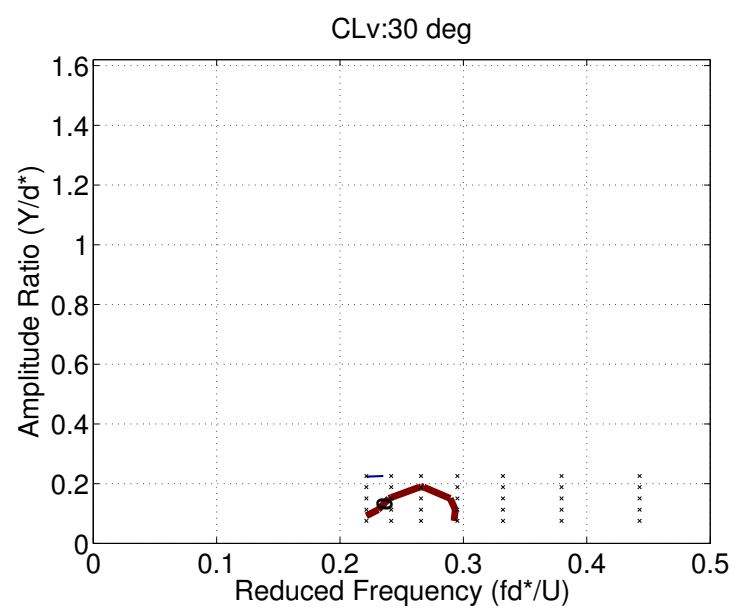

(c)

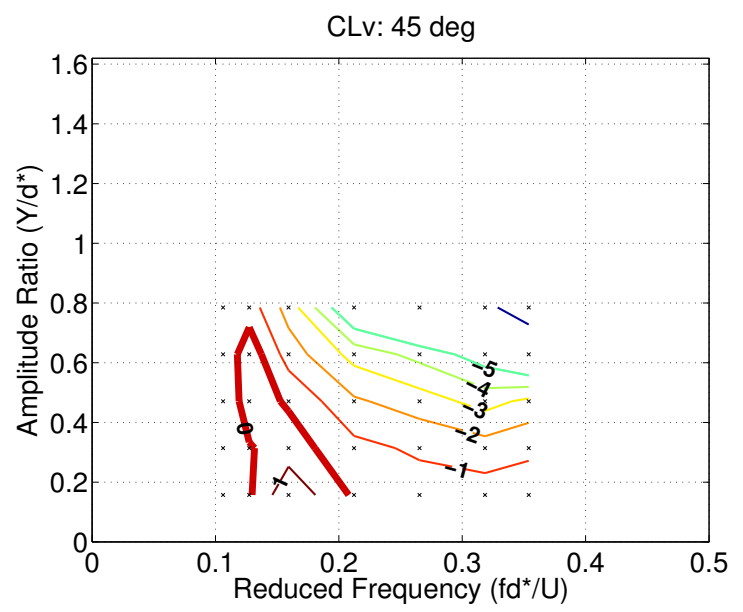

(e)

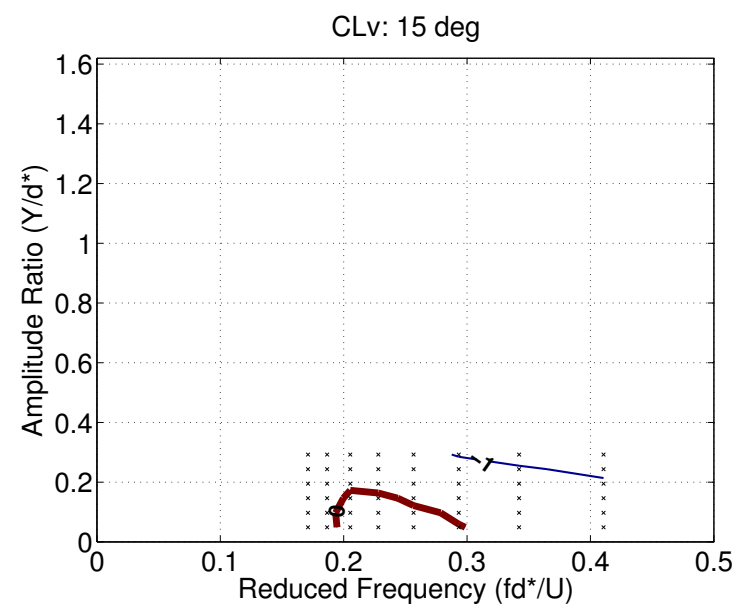

(b)

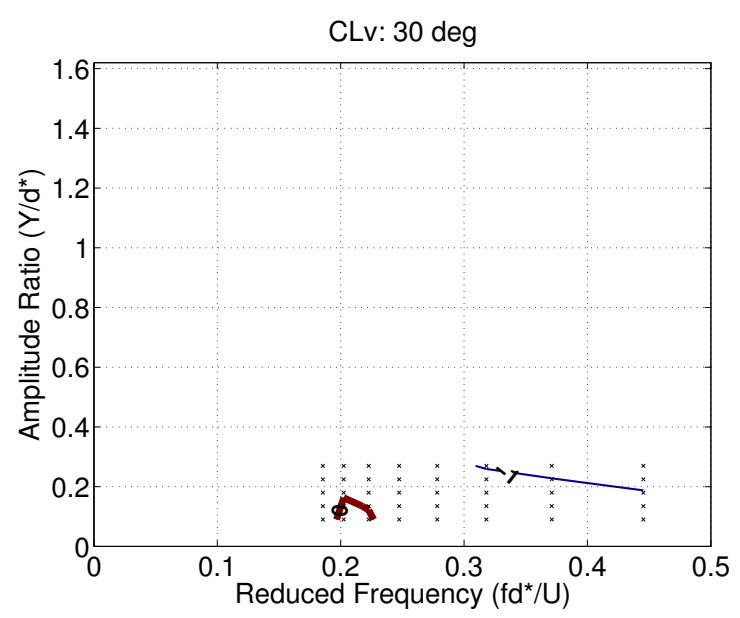

(d)

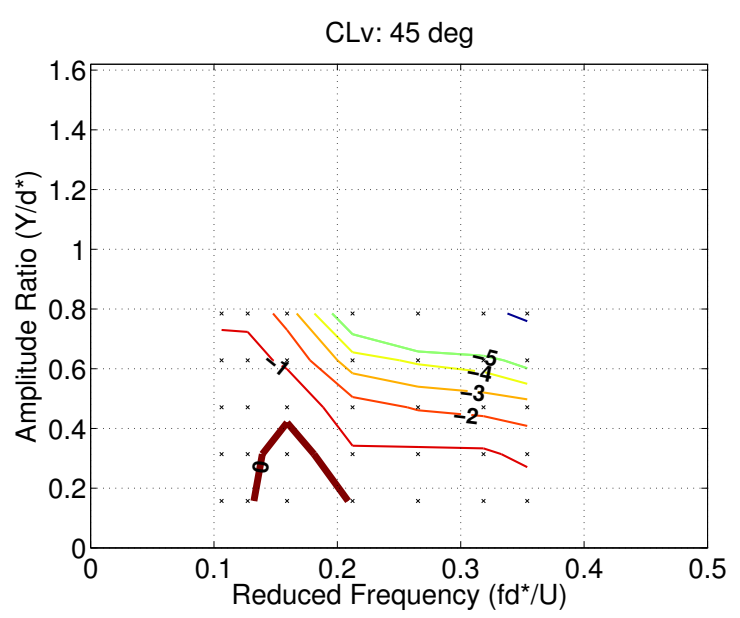

(f) 


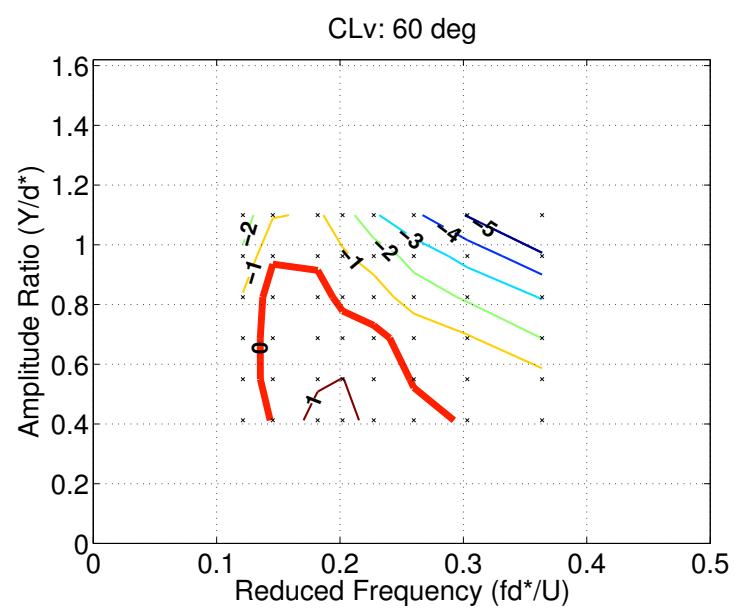

(a)

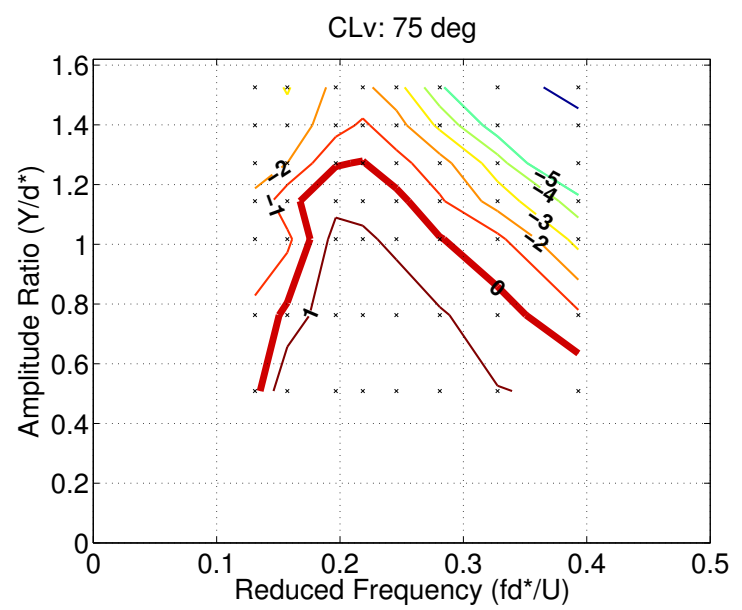

(c)

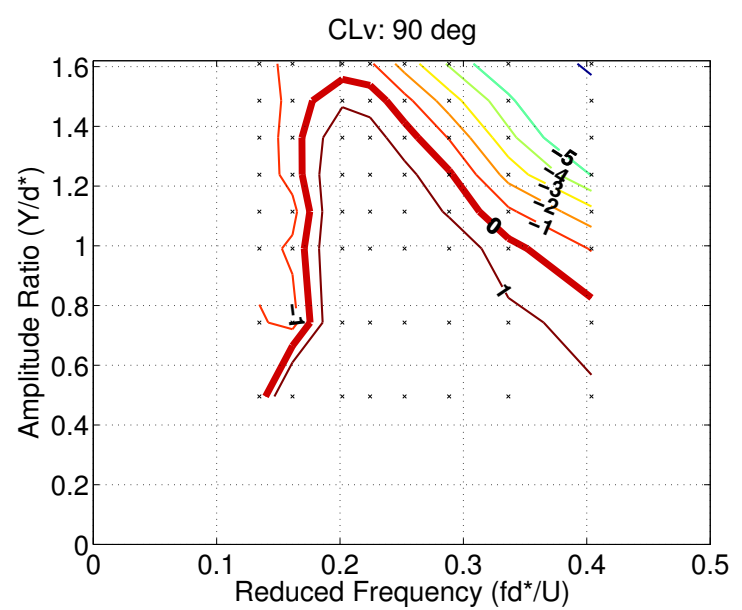

(e)

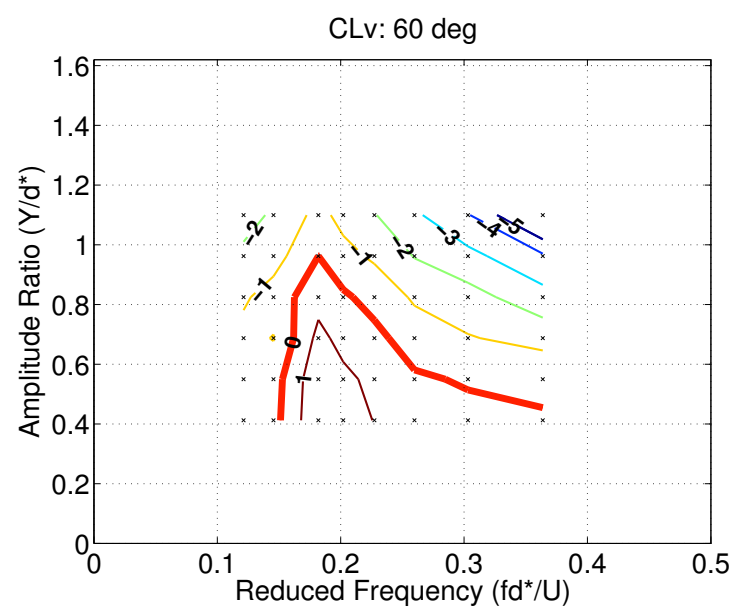

(b)

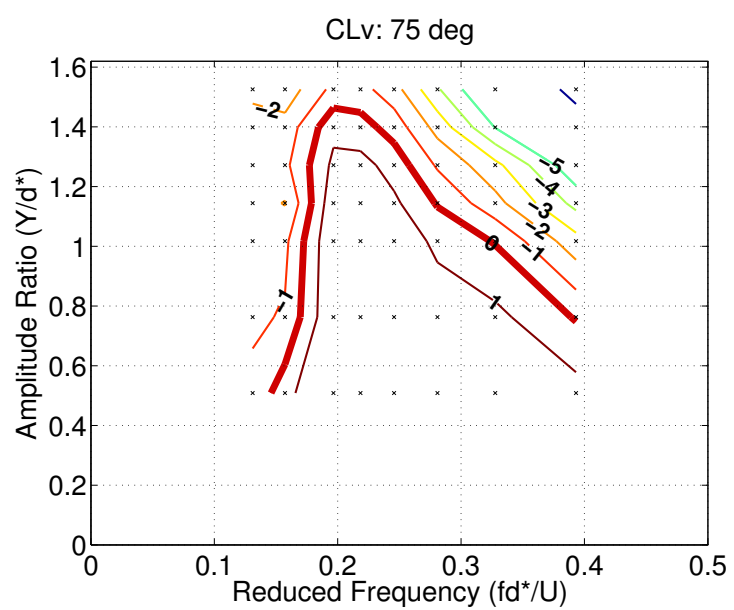

(d)

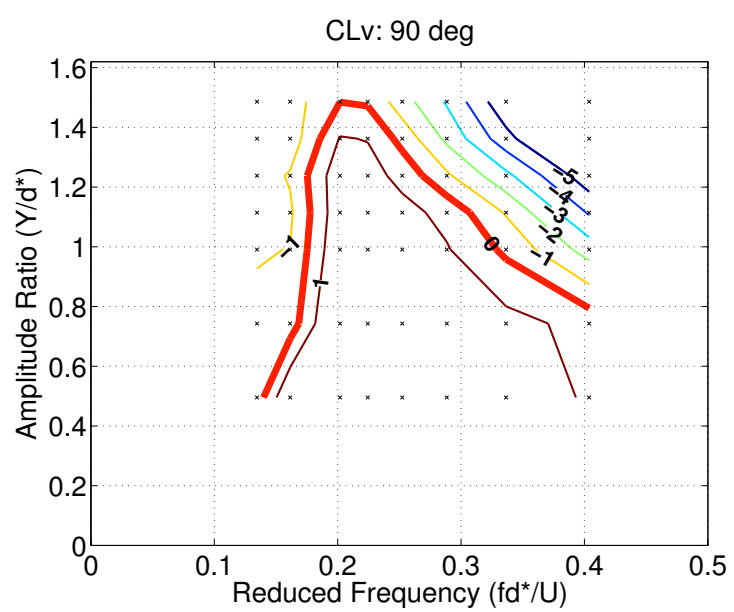

(f)

Figure 2-14: $C_{l, v}$ contour for (left column) ellipse and (right column) whisker at (a,b) 15; (c,d) 30; (e,f) 45; (g,h) 60; (i,j) 75; (k,l) 90. Contour levels are in increments of 1. 


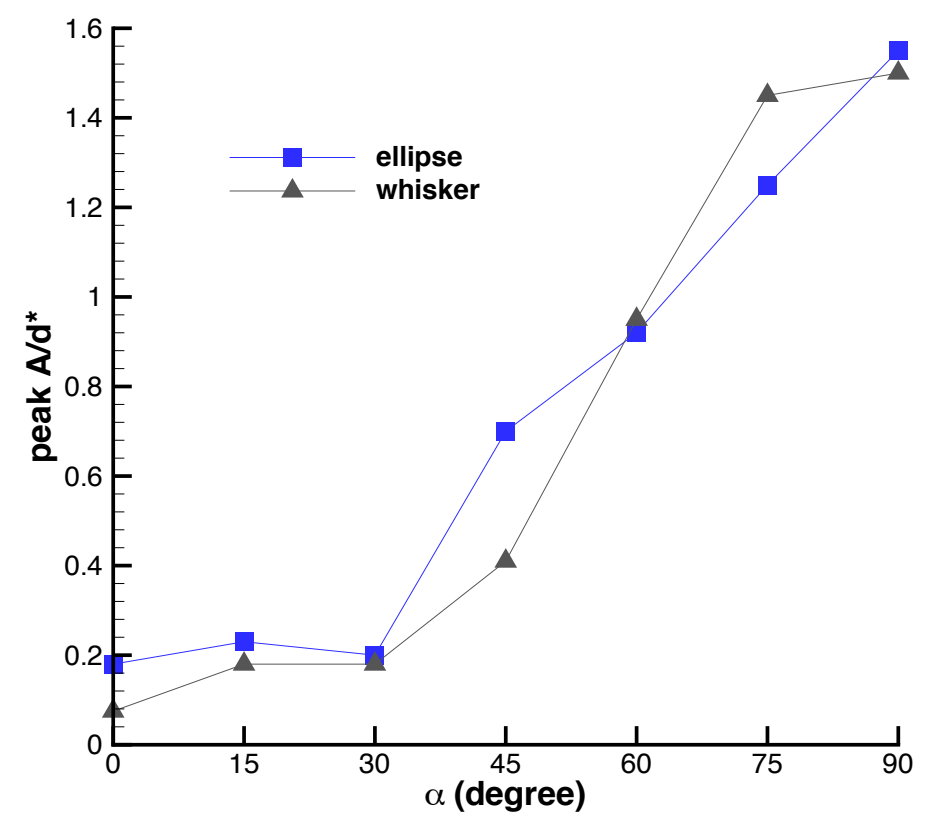

Figure 2-15: The peak amplitude of vibration for the elliptical cylinder and whisker increases as the angle of attack increases. $R e=5,300-7,030$ from $\alpha=0^{\circ}-45^{\circ}$ and $R e=2,530-3,210$ from $\alpha=60^{\circ}-90^{\circ}$.

\subsubsection{PIV}

\section{Steady Flow}

Flow visualizations of the whisker and ellipse being towed rigidly down a tank at various angles is shown in Figure 2-17. The whisker is seen to desynchronize the wake more than the the ellipse, especially at low angles of attack $\left(0,15,30^{\circ}\right)$. The vortex structures right next to the body is elongated, which reduces the oscillatory effect of the normal alternating vortex shedding pattern. At higher angles of attack $\left(45,60,90^{\circ}\right)$, the wake pattern looks similar between the two models, however the wake width is narrower for the whisker, especially in the $90^{\circ}$ case.

\section{1-D Free Vibrations - Dye Visualizations}

1-D free vibration experiments with the $30 \mathrm{x}$ scale whisker model were conducted by my colleagues Yahya Modarres-Sadeghi and Banafsheh Seyed-Aghazadeh at UMass- 


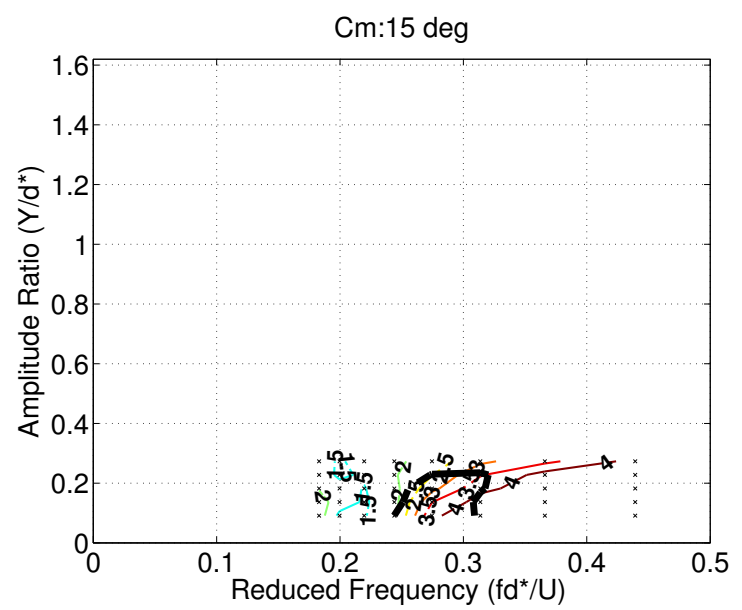

(a)

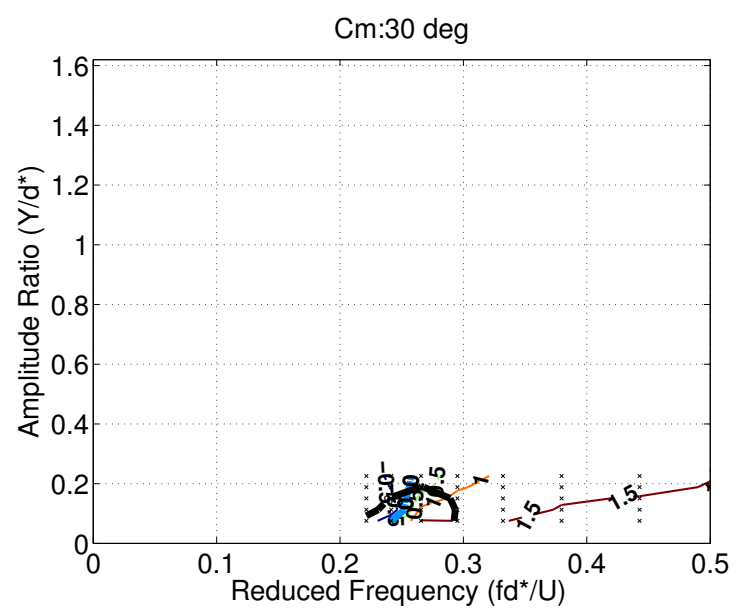

(c)

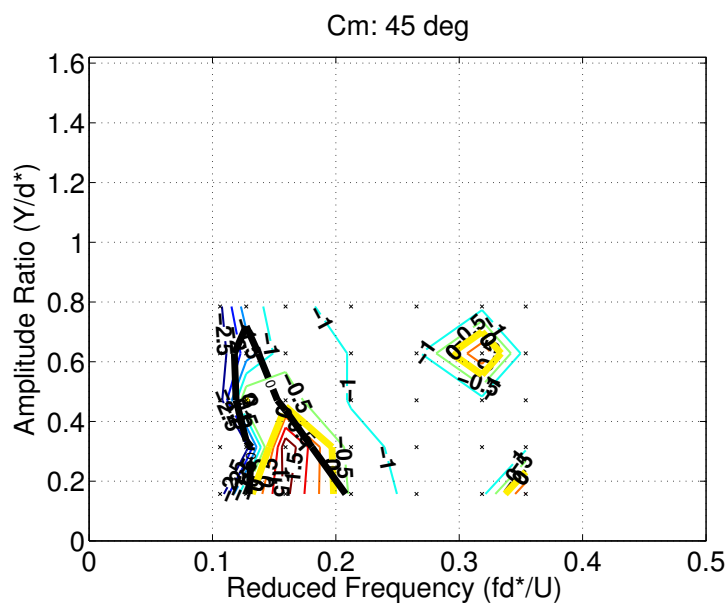

(e)

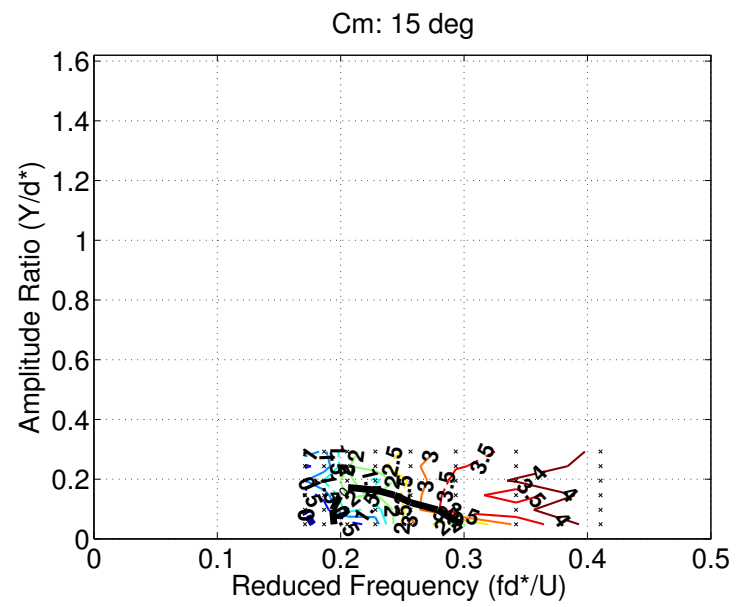

(b)

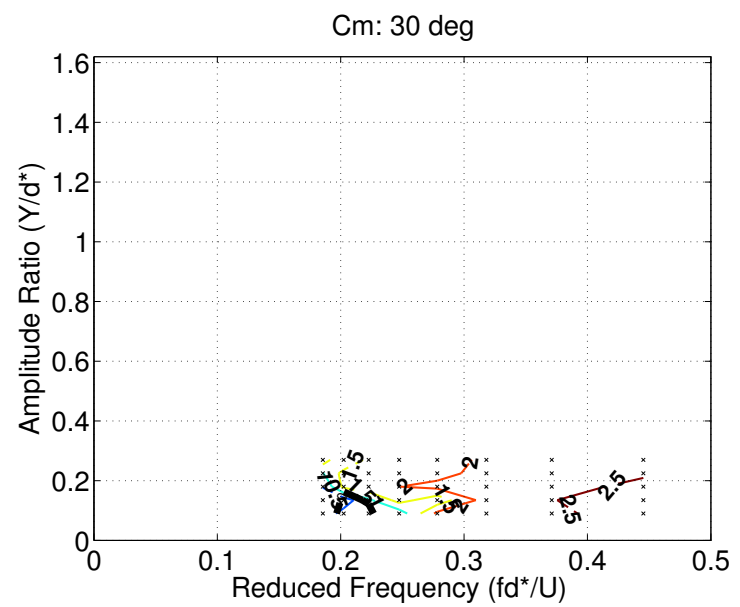

(d)

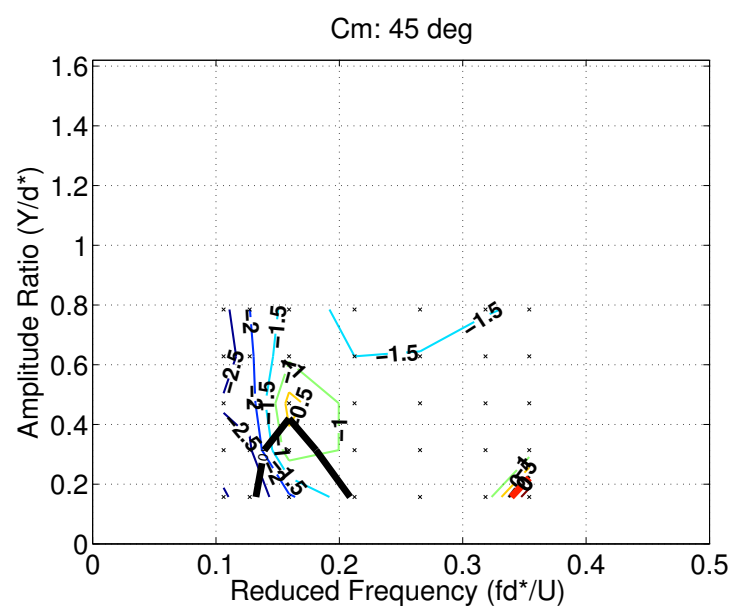

(f) 


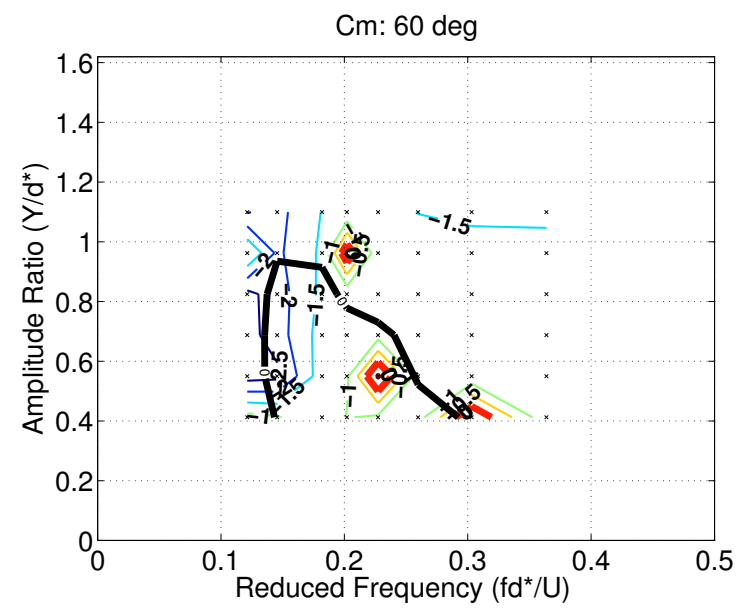

(a)

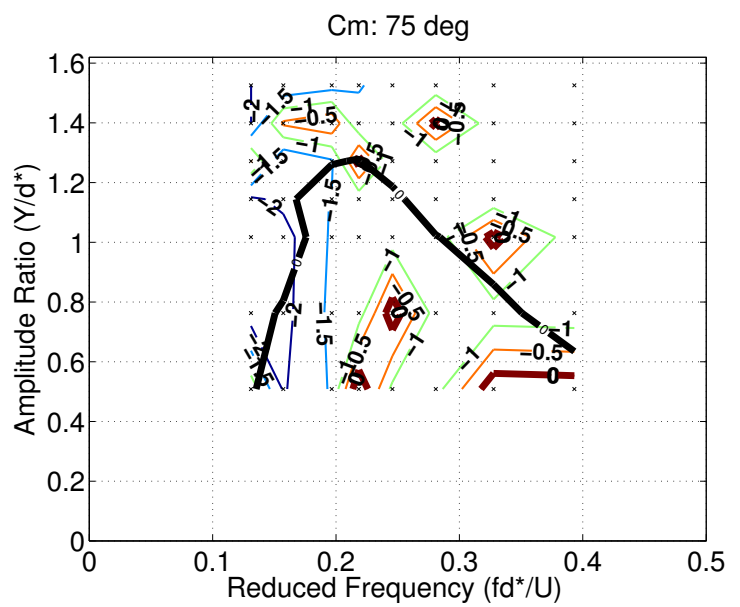

(c)

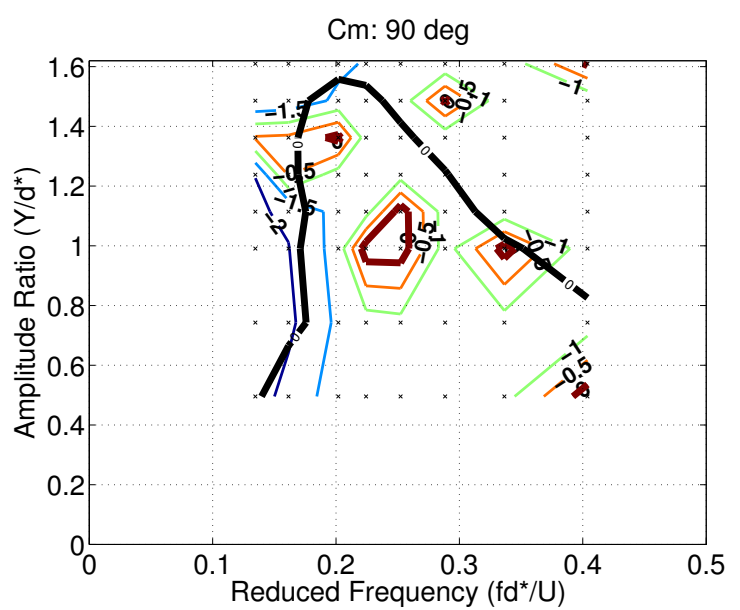

(e)

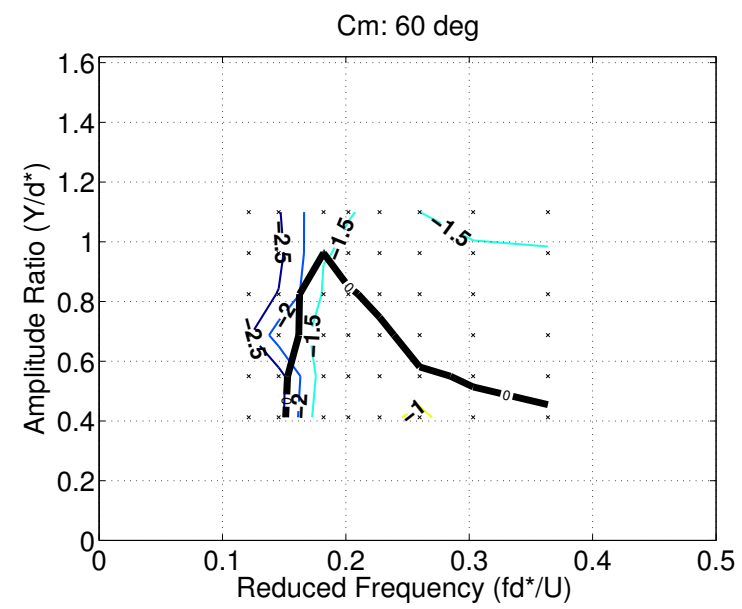

(b)

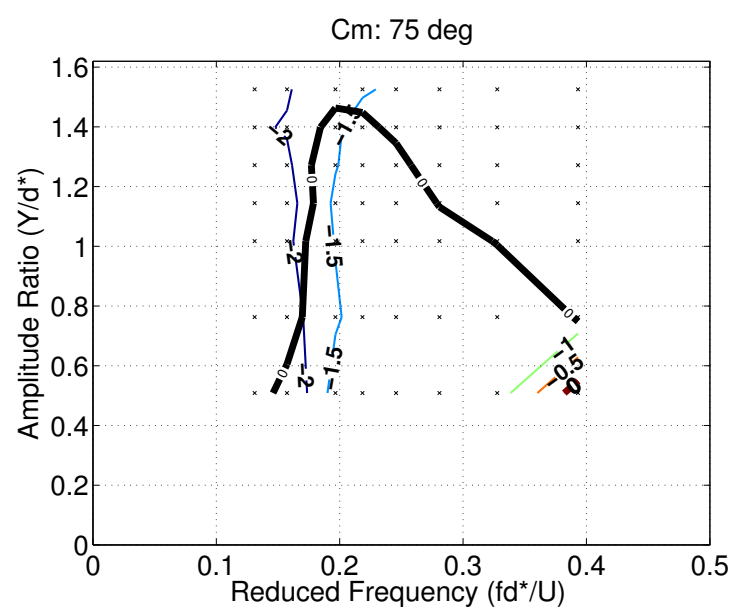

(d)

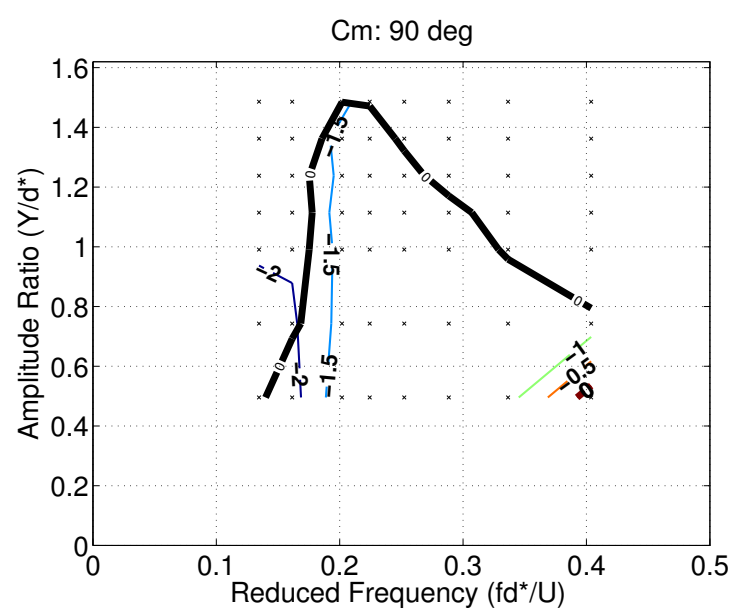

(f) Whisker 90

Figure 2-16: Effective added mass coefficient during forced vibrations for (left column) ellipse and (right column) whisker at (a,b) 15; (c,d) 30; (e,f) 45; (g,h) 60; (i,j) 75; $(\mathrm{k}, \mathrm{l}) 90^{\circ}$. Contour levels are in increments of 0.5. 


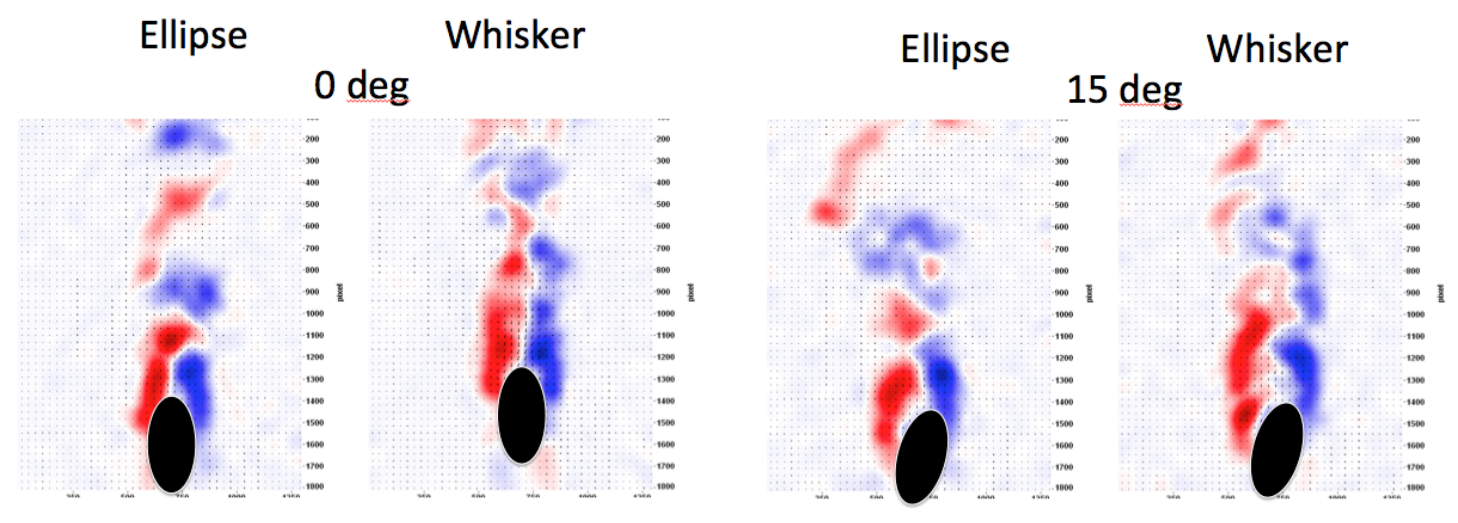

Ellipse

Whisker

$30 \mathrm{deg}$
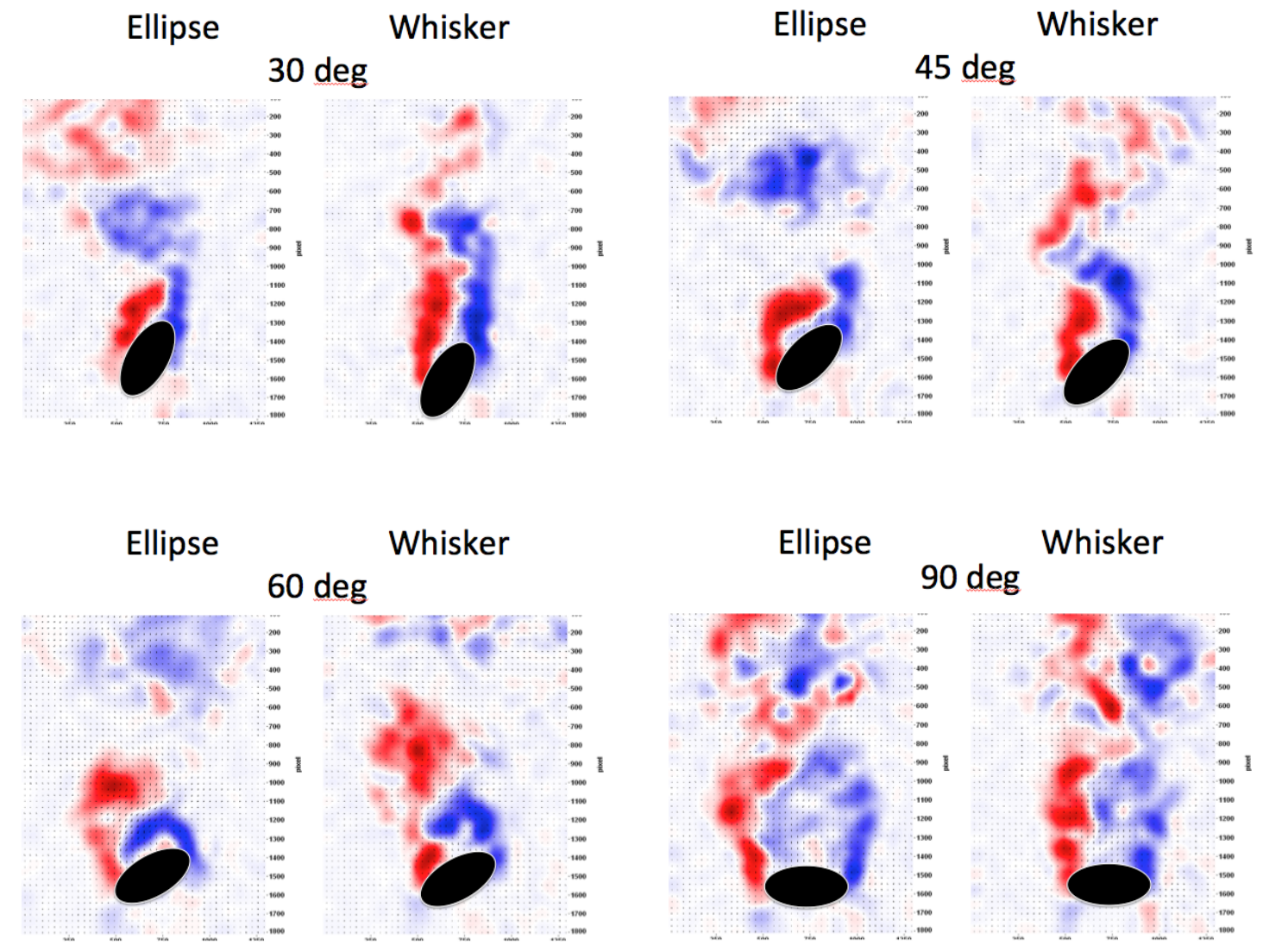

Figure 2-17: PIV of the wake behind an elliptical cylinder (left) and whisker (right) being rigidly towed at different angles of attack. $R e=5,300$. 

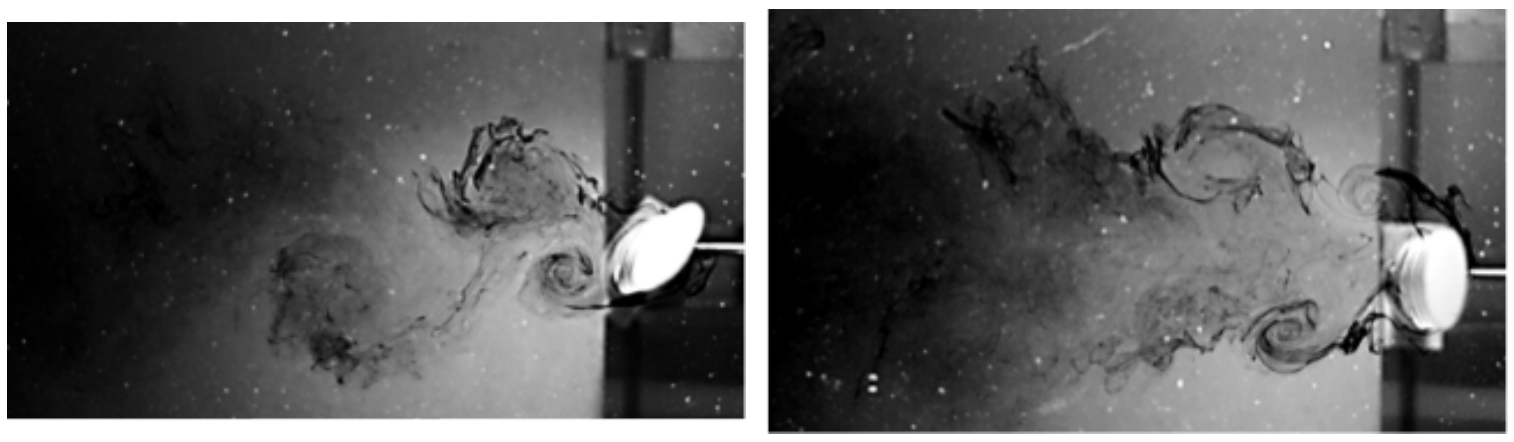

Figure 2-18: The wake shed behind the freely vibrating whisker for the (left) 45 and (right) $90^{\circ}$ cases. (Experiments conducted by B. Seyed-Aghazadeh and Y. ModarresSadeghi)

Amherst through use of a linear air bearing stage. Snapshots from dye visualizations of the wakes are shown in Figure $2-18$ for the whisker at $\alpha=45^{\circ}$ and $90^{\circ}$. Similar to the steady PIV results shown in Figure 2-17, the wake under these high angle conditions is characterized by coherent vortices and a large wake width.

\subsection{Discussion}

\subsubsection{Whisker in Frontal Flows}

The harbor seal whisker geometry has been dynamically tested, confirming the statement originally put forward by [58] that the geometry passively reduces VIV. Experiments of 1-D forced vibrations are used to find the zero contour of $C_{L v}$. The peak value of this contour indicates a factor of 4 and 10 lower amplitudes of vibration for an elliptical cylinder and whisker, respectively, in comparison with a baseline circular cylinder at the same Reynolds number. There is some variation in the whisker's $C_{L v}$ value over time. This variation is a result of the whisker requiring a longer time to reach lock-in than the cylinder or elliptical cylinder.

The effective added mass coefficient and damping coefficient indicate that the whisker geometry reduces the lock-in region compared to the elliptical cylinder. For both of these geometries, the still-water effective added mass in the y-direction is large because of vortex shedding along the body, leading to much smaller forcing terms in 
phase with the acceleration of the body and therefore smaller variation in the effective added mass. Both shapes exhibit large damping and small amplitudes of motion when excited in the y-direction because of their elongated body shapes, however the whisker has a much smaller bandwidth for excitation. Flow visualizations show that lock-in is reduced as a result of the desynchronized wake and increased formation length.

\subsubsection{Whisker at Angles of Attack}

VIV reduction does not apply when flow approaches the whisker from larger angles of attack. Beyond $\alpha=45^{\circ}$, the whisker responds similarly to a smooth elliptical cylinder, which is with an amplitude higher than that of a circular cylinder. At $\alpha=90^{\circ}$, the whisker vibrates 1.5 times its projected diameter. Flow visualizations show that the undulatory features that desynchronize vortex shedding in the whisker's streamlined configuration are too small relative to the bluff dimension to enable the same effect. The wake looks similar to that of the elliptical cylinder at the $\alpha \geq 45^{\circ}$ cases, which has strong coherent vortices.

The high VIV response as the body becomes more bluff with respect to the flow removes the possibility of using this shape as a vibration suppression device in flows with varying angle. Designs of whisker-inspired geometries that are omnidirectional were made in an attempt to create a shape that experiences low VIV regardless of the angle. See Appendix B for details of this study. These models were, however, unsuccessful at re-creating the extreme reduction capabilities of the whisker, highlighting the importance of the elliptical cross section in reducing VIV.

Although the whisker geometry is not suitable for use as a VIV reduction in cases where flow will approach the object with an $\alpha>45^{\circ}$, it holds great promise for use as a flow sensor. The VIV reduction in frontal flows allows easier distinction of flows that approach with a side component. Both seals and engineered devices could use this for heightened sensing of the direction of oncoming flow. This evidence has encouraged sensor development, which is described in the first half of Chapter 3 . 


\subsubsection{Note on the geometry}

This data is intended to describe the VIV properties of a "whisker-inspired" geometry. The geometry used here can likely be further optimized for VIV reduction. In fact, it was noticed that the morphology of the harbor seal whisker (in both real whisker specimens obtained from the New England Aquarium as well as the specimen reported in [45]) is different than the geometry reported in [58], which was replicated in this chapter. The undulation wavelength appears to be nearly double the wavelength used here. In Chapter 4 both the current geometry and one that captures the longer wavelength will be tested. 


\title{
Chapter 3
}

\section{Flow Sensor Design}

\author{
How can these unique vibration properties be realized in a \\ physical prototype?
}

\subsection{Introduction}

It is desired to develop a sensor that mimics the capability of harbor seals to detect vortex wakes. Since the mechanism by which the seals do this is unknown, we approach this goal in steps. First, a device that conducts basic steady flow measurements is created. It is designed to measure flow velocity and exploit the discovered seal whisker vibration properties (Chapter 2) for heightened flow direction sensing. Second, the design is refined and adjusted for use as a platform to systematically explore wake-detection capabilities. That detailed study is then described in Chapter 4.

The current work utilizes the discovered mechanism of frontal-flow vibration reduction to achieve clear drag-based readings for correlation to oncoming flow velocity. Towing tank tests are used to measure the sensor's range of vibrational response over various flow directions. Field experiments test the sensor's usability at sea. Finally, a refined device is put forth as a platform to conduct tests on wake detection. 


\subsubsection{Previous Work}

\section{Flow Velocity Sensors}

The first step seeks to create a device that measures flow velocity. A variety of such sensors already exist. These span the use of acoustic-, magnetic-, mechanical-, and pressure-based sensing techniques. AUVs generally utilize Doppler velocity logs (DVLs), which measure velocity based on travel time of acoustic pings. These active devices demand a significant portion of the vehicle's battery power. Experiments with real seals suggest that they may use their vibrissae to detect and maintain their speed [111]. A whiskerlike sensor, which is mechanical-based as in the real seals, could serve as a low-power, low-cost alternative to the standard devices in certain scenarios.

Previous whiskerlike or hairlike flow sensors have been developed [6, 32, 52, 127]. The "whiskers" used have generally been limited to cylindrical rods. The drag-based response of these rods can indeed be used to determine flow velocity and direction.

\section{Vortex Wake Detection Sensors}

An important limitation to the existing whiskerlike and hairlike flow sensors is that their uniform circular cross section precludes them from detecting unsteady flow features. Since such bluff bodies experience significant vortex-induced vibrations above $R e \approx 50$, the baseline signal they emit will include strong oscillations, which can reach amplitudes of about one cylinder diameter [49]. Any additional response upon encountering features such as vortex wakes may then be indistinguishable from the baseline vibrations.

Existing flow sensors could be modified to directly target measurement of vortex wakes by actively emitting signals, such as acoustic-based ones. Passive measurement options do exist through instruments such as hot-wire anemometers and pressure probes. Such devices have often been used in laboratory settings to measure flow velocity and can be used to detect vortices [36, 113, 126].

The remaining barrier is in conducting passive detection while moving forward at significant speed. Devices reaching into the flow while mounted externally to an AUV 
would need to function despite the presence of steady oncoming flow, which can lead to VIV on the sensor. This work therefore seeks to develop an instrument for passive vortex wake detection via high wake-"signal"-to-baseline-"noise" response.

\subsection{Flow Sensor - Version 1}

The design, calibration, and testing of the Version 1 device was first presented in [10] and is described again here.

\subsubsection{Design}

This device was designed to operate with a similar sensing mechanism to that used by the real seals. No nerves are present inside the length of the real whiskers. Instead, the sensing takes place first in the seals' cheek as the whiskers bend in the flow. Similarly, the sensor here was designed to measure deflections at the base of the whisker.

The complete sensor design is shown in Figure 3-1. The whisker section is the same model used in Chapter 2 for the larger angle of attack cases. This part was fabricated via stereolithography at a scale of thirty times the real whisker diameter (whose dimensions are taken from [58]), giving it an average crossflow diameter $(d)$ of $1.59 \mathrm{~cm}$, when facing the flow in the streamlined direction. The length of the whisker section was chosen as $27.5 \mathrm{~cm}$, which encompasses five full wavelengths of undulations. The whisker protrudes from a housing, which contains Flexpoint Bend Sensors that change resistance based on the amount they are deflected. The underlying Bend Sensor configuration is based on previous work done by a colleague, whose design details are outlined in [130].

A closer view of the Bend Sensors is given in Figure 3-2a. The four are rigidly mounted at one end in a holder and allowed to bend at the other end as a square plate pushes on them. That plate is attached to the end of a rod that is connected to the whisker. The starting position of the plate is such that it is just touching each of the Bend Sensors, allowing any small deflection to produce a change in voltage output.

The sequence is: Deflection of the whisker pushes on the plate, which pushes on the 


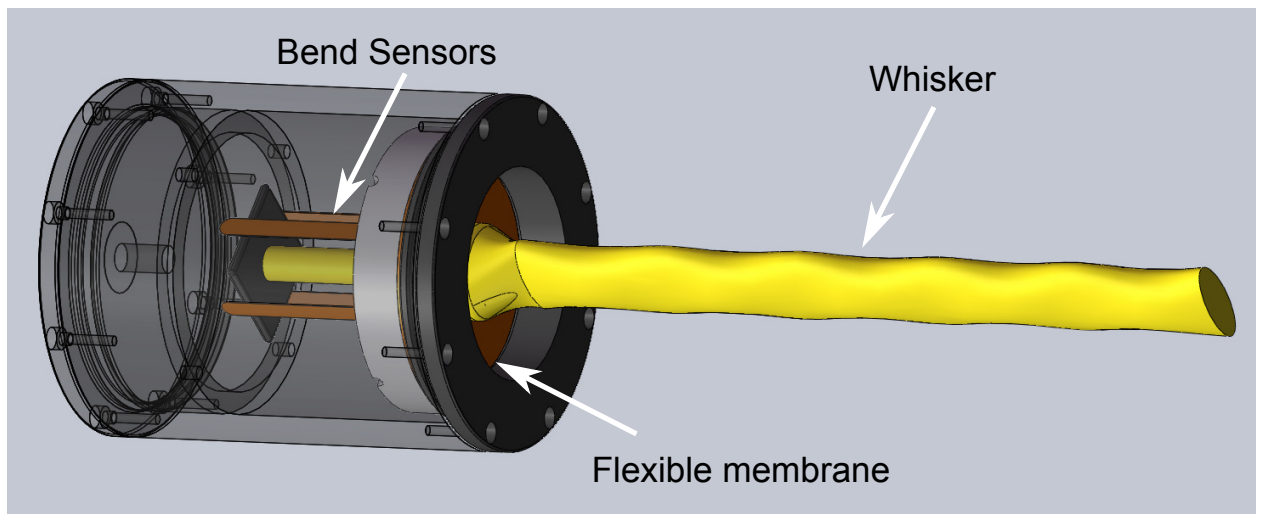

Figure 3-1: The whisker (yellow) mounted to a pressure housing, which contains four Bend Sensors (dark orange).

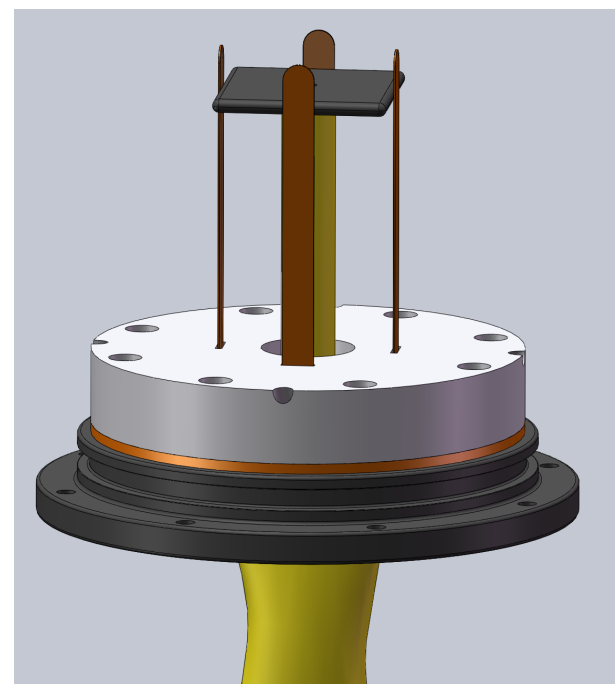

(a)

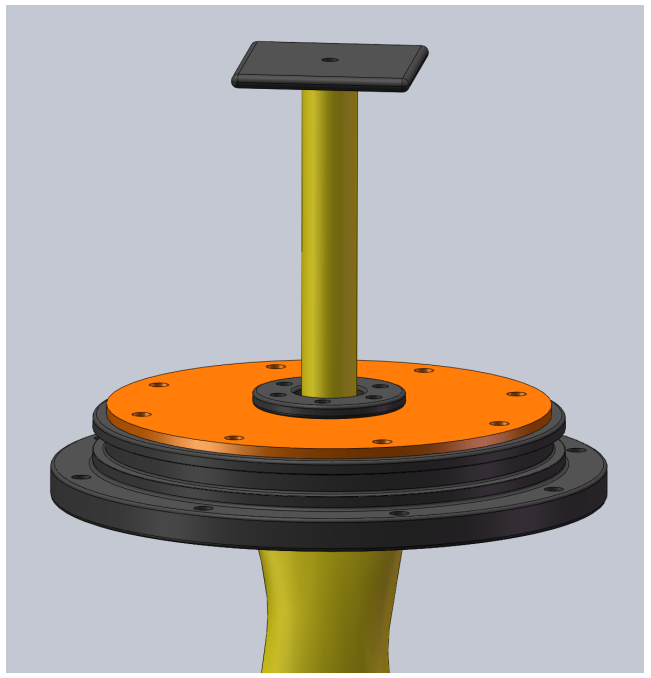

(b)

Figure 3-2: (a) A square plate (in black) at the end of a rod connected to the whisker pushes on the four Bend Sensors as the whisker deflects. (b) A silicone diaphragm (in orange) between the whisker and a retaining ring allows flexible motion.

Bend Sensors, which change resistance and thereby provide deflection information. This section describes how the magnitude of the output can then be translated to direction and velocity of the oncoming flow.

An interesting design parameter was encountered in requiring the whisker to flexibly move in response to the oncoming flow but simultaneously maintain a watertight seal with the housing. The chosen solution was to use a flexible base with the dual function of allowing movement of the whisker and serving as a waterproof barrier to 
the pressure housing. A silicone diaphragm (Shore 50A hardness) was sandwiched between the end of the whisker and a plastic piece (Figure $3-2 \mathrm{~b}$ ) via bolts. The whisker is thus firmly attached to the rest of the system, but the diaphragm allows motion. The lack of holes from outside to inside the housing allows for a watertight seal.

This flexible membrane will deform under water pressure when the sensor is submerged. Such a shape change would cause additional changes in readings from the Bend Sensors. As such, it was chosen to fill the inside of the housing with oil, providing a back pressure to keep the diaphragm in a stationary position until the whisker deflects.

\section{Electronics}

A separate housing is used to store the electronics since the main housing is filled with oil. A standard pressure housing was built to hold an amplification circuit, a data logger, and a power supply. Wires from the Bend Sensors go from the main housing, through a Subconn connector and cable to the electronics housing, to the amplification circuit, and then into the data logger (Figure 3-3). The DATAQ DI-710 data logger records data onto an SD Card at a sampling frequency of $1440 \mathrm{~Hz}$. A battery pack is used to power the data logger.

A circuit that provides filtering and amplification to the Bend Sensors was designed and assembled. The diagram is outlined in Figure 3-4. LM324 op-amps are used in a slightly expanded version of a voltage divider. Resistor values were chosen to closely match the base resistance value of the corresponding Bend Sensor. Small capacitors are included in a RC low-pass filter configuration.

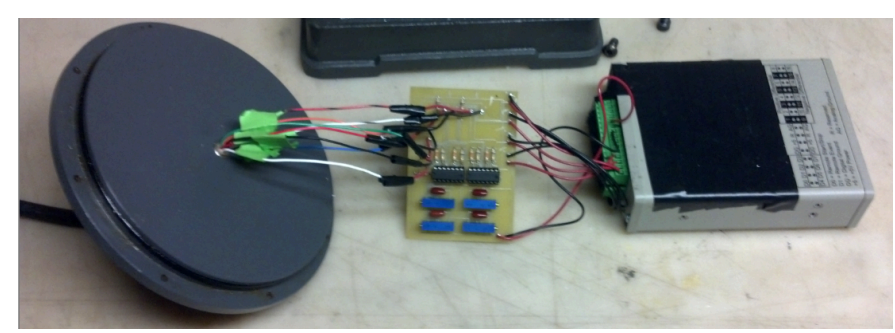

Figure 3-3: The electronics that are stored inside a separate housing: circuit and data logger. The battery pack is not pictured. 


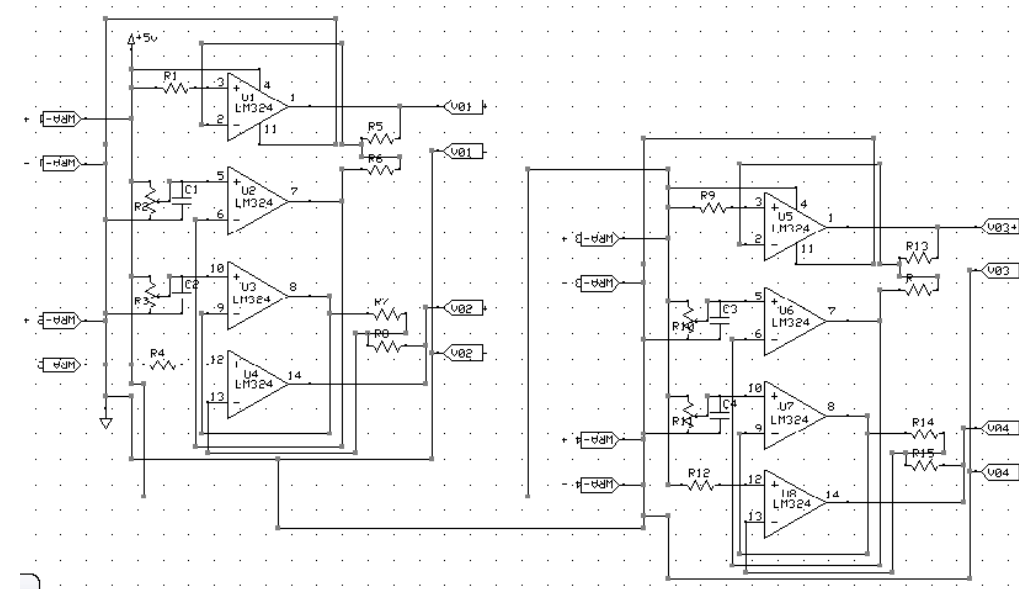

Figure 3-4: Circuit diagram. The Bend Sensor signal lines are filtered and amplified.

\subsubsection{Calibration}

The voltage output from the Bend Sensors was related to a physical deflection length. This was done by manually deflecting the whisker in equal increments and matching the output voltage to the corresponding tip deflection distance determined from the adjacent ruler. Figure 3-5 shows the setup. The sensor was then rotated $90^{\circ}$ and the procedure was repeated three more times to collect data for all four of the Bend Sensors.

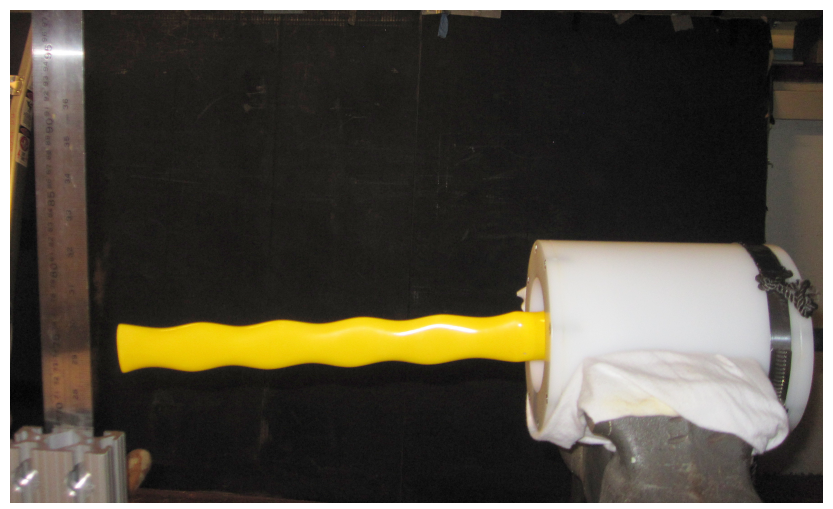

Figure 3-5: Setup for bench-top calibration. Deflection of the whisker was conducted in increments, using the ruler on the left as a reference.

Figure 3-6 shows the voltage output for each of the four Bend Sensors over the incremental deflections. Trendlines were best fit to these curves and the corresponding equations are shown in Equation 3.1. $V$ is voltage (Volts) and $d_{t}$ is tip displacement 

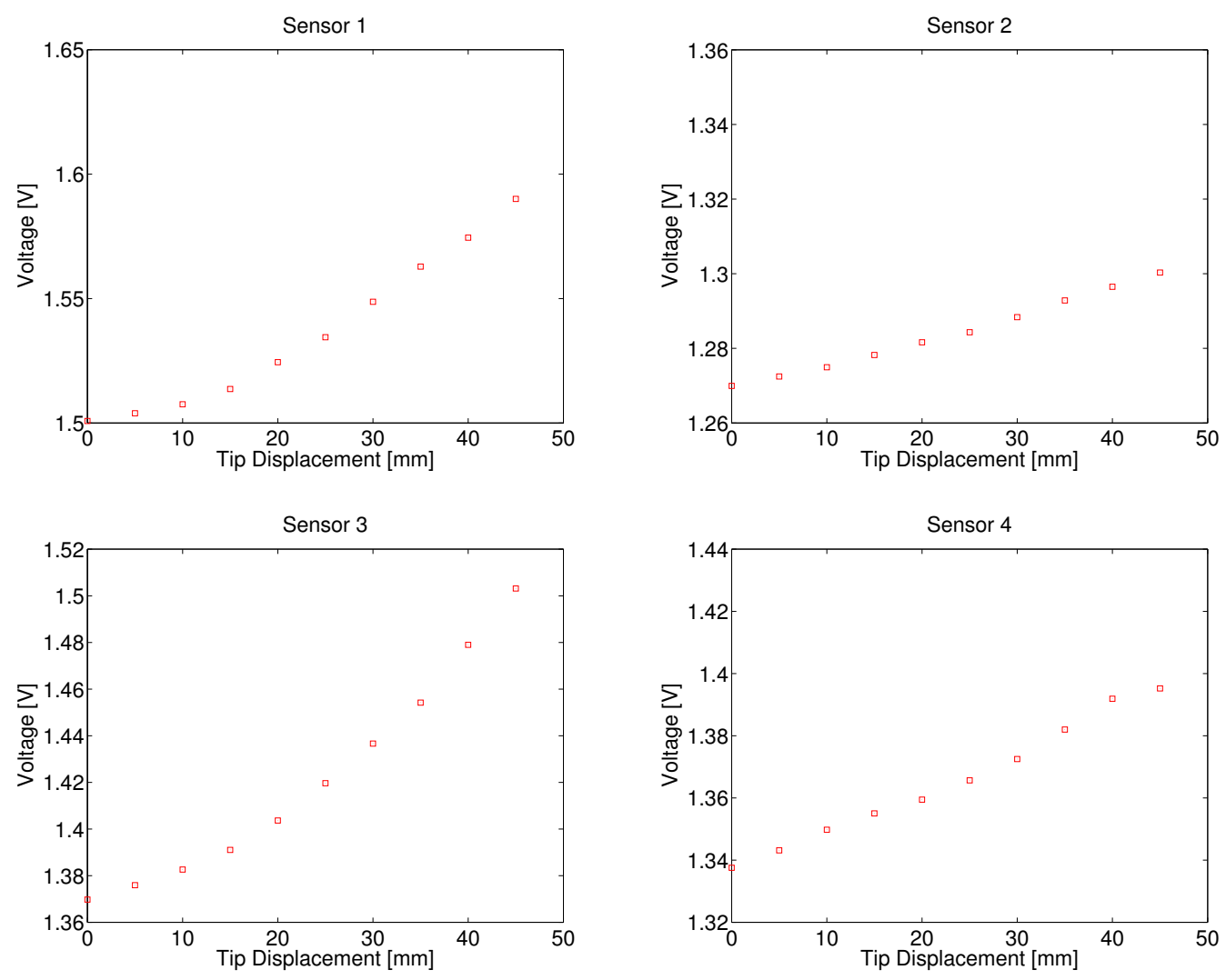

Figure 3-6: Voltage output versus whisker tip displacement for each of the four Bend Sensors.

(mm). Subscript numbers indicate which Bend Sensor the variable corresponds to.

$$
\begin{array}{rr}
V_{1}= & 0.0008 d_{t 1}^{2}+0.0015 d_{t 1}+1.4972 \\
V_{2}= & 0.0034 d_{t 2}+1.2651 \\
V_{3}= & 0.0013 d_{t 3}^{2}+0.0008 d_{t 3}+1.3683 \\
V_{4}= & 0.0065 d_{t 4}+1.3292
\end{array}
$$

The coefficient of determination $\left(R^{2}\right)$ for each of these equations is $0.997,0.993$, 0.999, and 0.989 in sequential order. Sensors 2 and 4 can be characterized by a linear relationship between voltage and displacement, but Sensors 1 and 3 are best described with a quadratic one. This variation from Bend Sensor to Bend Sensor emphasizes the need for quantifying the individual responses. 


\subsubsection{Towing Tests}

The whisker response (vibration and deflection) to various flow conditions was quantified and collected. This was done by conducting towing tests in the large tank of the MIT Towing Tank Lab, where the sensor was mounted to the carriage with the whisker model vertically submerged (Figure 3-7). It was towed at different velocities $(U=0.2,0.4,0.6,0.8 \mathrm{~m} / \mathrm{s})$ and different angles of attack $\left(\alpha=0,30,45,60,90^{\circ}\right)$. As described in the previous chapters, zero angle of attack refers to the orientation where the longer diameter is exactly in-line with the oncoming flow. The corresponding Reynolds number is $R e=3,180$ to 12,720 based on the average crossflow diameter $(d)$ at $\alpha=0^{\circ}$. Figure 3-7 also displays the orientation of the Bend Sensors. Note that as the angle of attack changes, the sensors which are in the crossflow and inline direction also change.

Plots of voltage versus time were generated for each run and compiled for later use as a lookup table to invert output voltage to the corresponding flow information. A selection of these runs are shown in Figure 3-8. Each towing run shows a change in voltage once the carriage begins moving forward, indicating a Bend Sensor deflection in that corresponding direction. Then oscillations develop in the crossflow Bend Sensors, and finally the voltage ramps back to the initial value as the carriage stops.

As discovered in Chapter 2, the whisker geometry vibrates preferentially with direction. As the angle of attack increases, the crossflow vibrations increase. At $\alpha=0^{\circ}$, the voltage of the crossflow sensors ( 1 and 2 , for this angle) oscillated with extremely small amplitude (Figure 3-8a). The crossflow sensors in the $90^{\circ}$ case (3 and 4), however, show significant oscillations (Figure 3-8f). The inline sensors for $0^{\circ}$ and $90^{\circ}$ experience a mean deflection but very little oscillations (Figures $3-8 \mathrm{~b}$ and $3-8 \mathrm{e}$. For angles between those two extremes, such as $45^{\circ}$, the crossflow oscillations are captured in a combination of all four Bend Sensors (Figures 3-8c and 3-8d). 


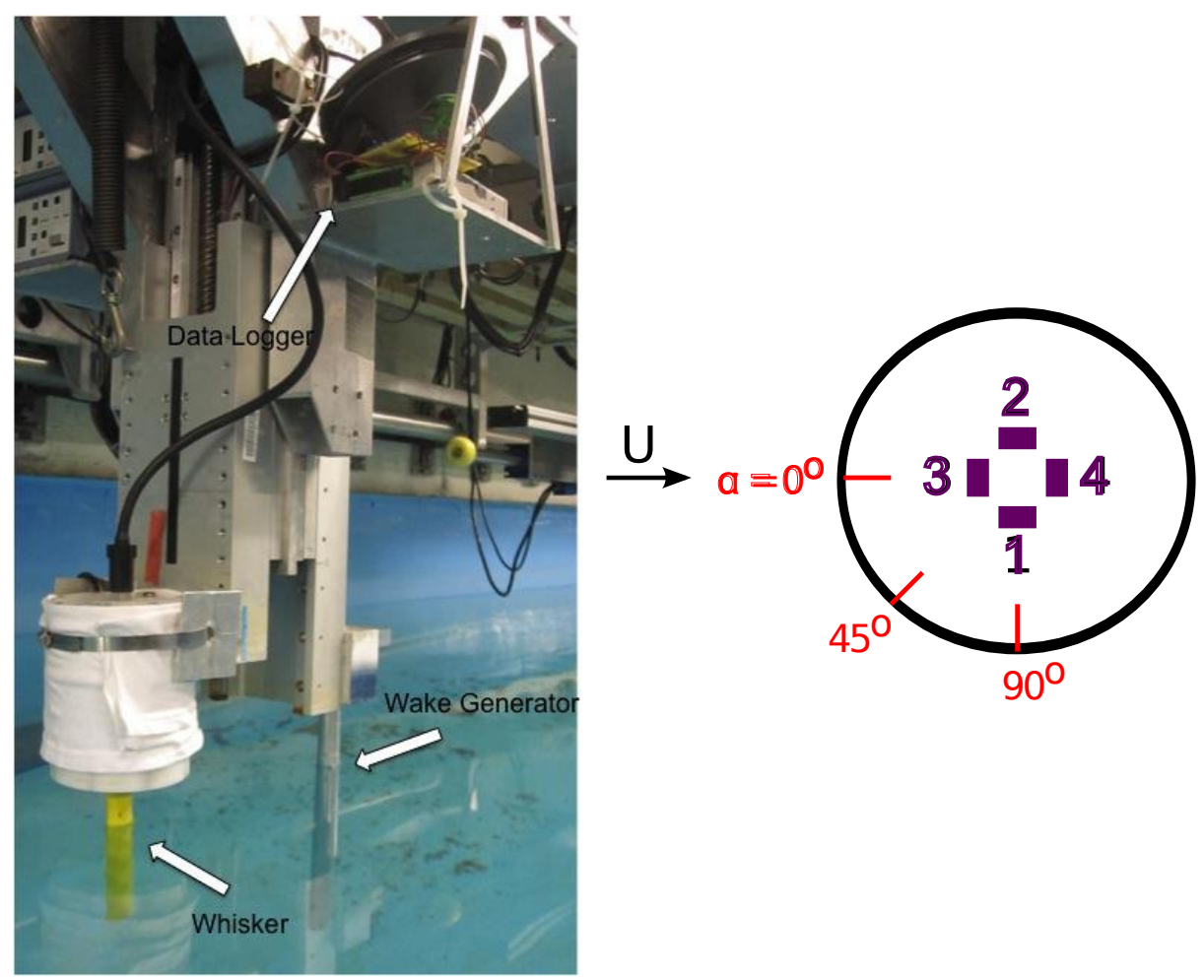

Figure 3-7: Towing test setup. (Left) The sensor is mounted to the carriage, and a data logger records voltage output. Note: The wake generator (circular cylinder) pictured was not used for the experiments shown in this Chapter. (Right) Schematic of the inside of the sensor housing (top view). The Bend Sensors (purple) are numbered with this convention throughout. Note: For $\alpha=0^{\circ}$, Bend Sensors 1 and 2 are in the crossflow direction, but as $\alpha$ is increased to $90^{\circ}$, Bend Sensors 3 and 4 become fully in the crossflow direction.

\section{Sensing Procedures}

The results from the calibration runs are then organized so that a given sensor reading can be converted to the corresponding flow velocity and direction. This is done by first extracting three main features from the calibration data: deflection in the drag direction and vibration amplitude in both axes. These three variables are converted from voltage to a physical length of motion via Equation 3.1 .

The oncoming flow angle can be determined by comparing vibration magnitudes in the two axes. The Bend Sensor that lies in the crossflow direction will vibrate with higher amplitude than the adjacent Bend Sensor. Figure 3-9a depicts this trend of diverging values between the inline and crossflow motions as the angle of attack in- 


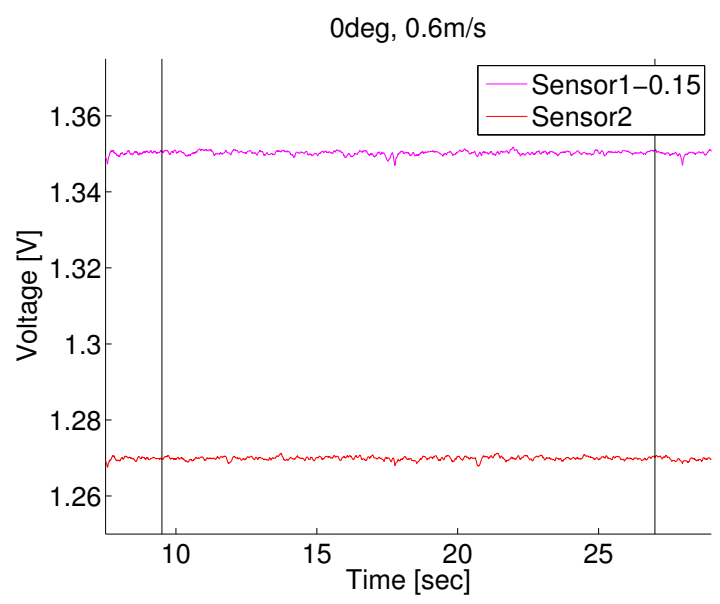

(a)

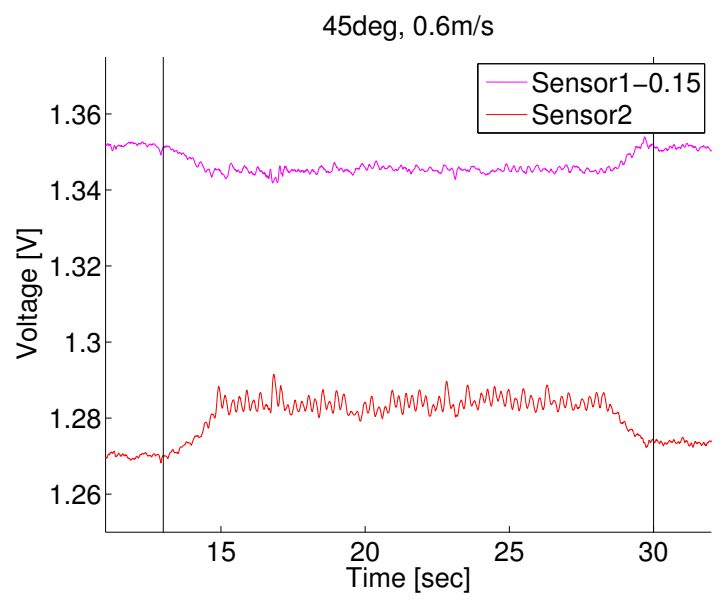

(c)

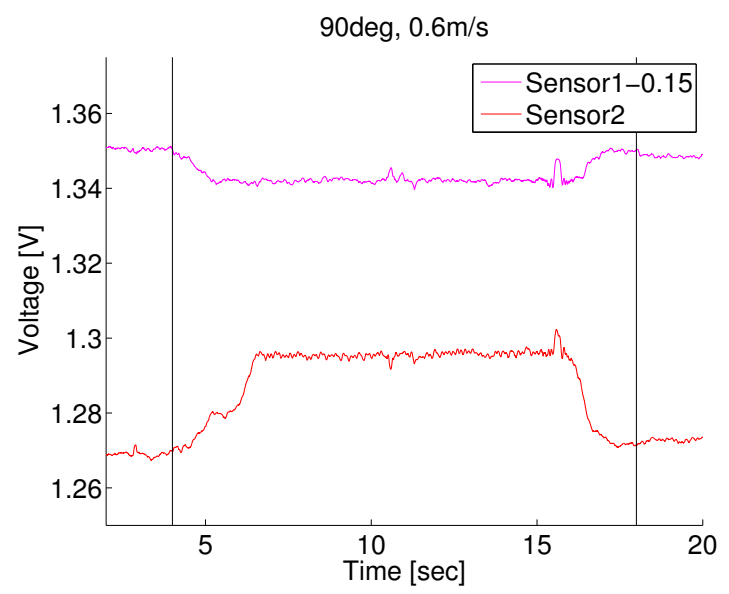

(e)

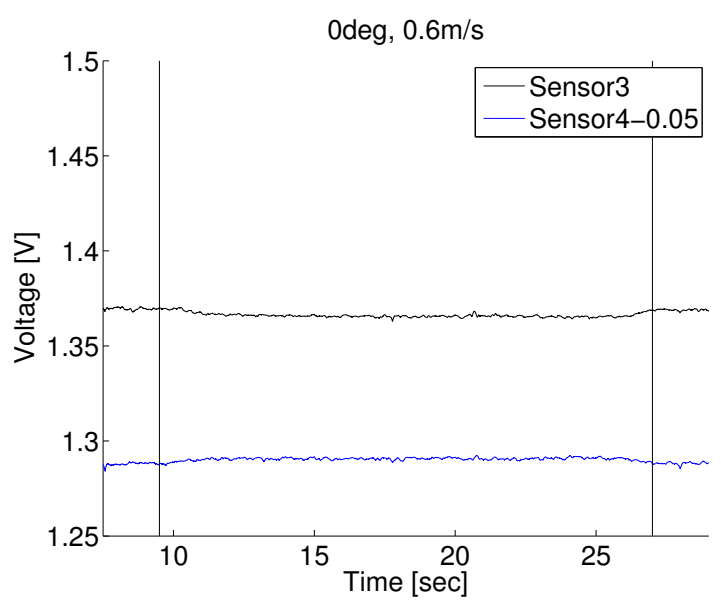

(b)

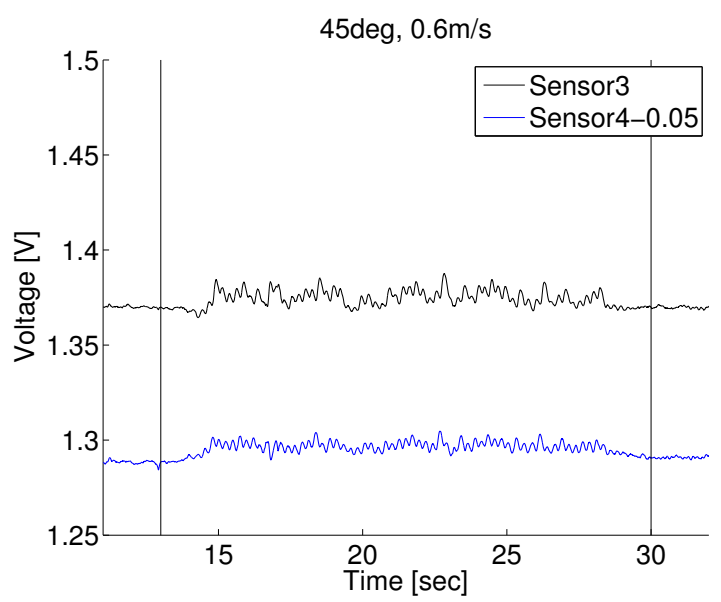

(d)

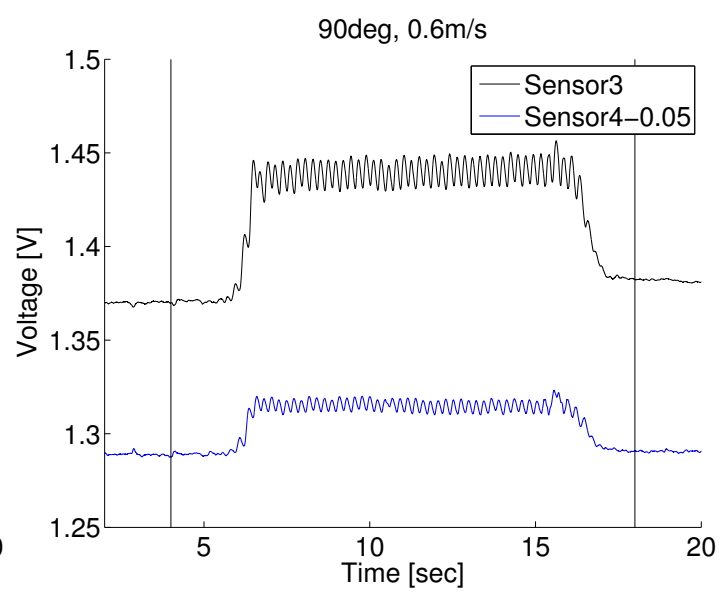

(f)

Figure 3-8: Time traces of the Bend Sensor outputs. Black vertical lines indicate start and end of the carriage motion. (Top row) $\alpha=0^{\circ}$ : minimal change occurs. (Middle row) $\alpha=45^{\circ}$ : ramps and oscillations in voltage are seen. (Bottom row): $\alpha=90^{\circ}$ : larger oscillations occur in the crossflow (Sensors 3,4) and larger ramps occur inline (Sensors 1,2). Sensors 1 and 4 are shifted by a constant value to consolidate plots. 
creases. Note that the values presented here are not universal values for the whisker's response. The exact values will change with the mass-damping $\left(m^{*} \zeta\right)$ value of the setup. A more quantitatively accurate comparison is found through the forced vibration data shown in Chapter 2, The plots here are intended merely to confirm the trends.

A comparison of the two amplitudes therefore provides the first piece of information: angle of oncoming flow. However, it is important to point out that the vibration amplitudes vary as the flow velocity changes. The divergence between in-line and crossflow amplitudes remains across all velocities tested here.

The amplitude values shown here were determined from the corresponding Bend Sensors for that particular angle of attack (i.e., Sensor 2 for crossflow vibration of $\alpha=0^{\circ}$ and Sensor 4 for crossflow vibration of $\alpha=90^{\circ}$ ). For calibration runs conducted at $\alpha=30^{\circ}, 45^{\circ}$ and $60^{\circ}$, no Bend Sensor was aligned exactly with either crossflow or in-line flow directions. Measurements in the in-line direction were therefore approximated by $A($ Sensor $X)=\frac{A(\text { Sensor } 1)}{\sin \alpha}$ and measurements in the crossflow direction were approximated by $A($ Sensor $X)=\frac{A(\text { Sensor } 4)}{\sin \alpha} \cdot A($ Sensor $X)$ is the amplitude of motion that would be experienced by a Bend Sensor appropriately aligned with the corresponding angle of attack $(\alpha)$. This geometric relation converts the motion $(A($ Sensor 1$)$ or $A($ Sensor 4$))$ measured by Sensors 1 or 4 , whose locations are shown in Figure 3-7, to an estimated value.

Next, the sensor can be used to determine the flow velocity $(U)$. As shown in Figure $3-9 \mathrm{~b}$, the inline deflection magnitude (found from the aft Bend Sensor in each case) increases with velocity. It is also dependent on angle of attack, because as $\alpha$ increases, the area facing the flow becomes less streamlined. As such, higher-angle cases display a higher in-line deflection. So once the angle of attack is determined, the output voltage in the in-line direction can be directly converted to flow velocity.

\section{Accuracy}

Based on the bench-top calibration, the sensor has a resolution of approximately $1 \mathrm{~mm}$ deflection at the whisker base. Correspondingly, it can safely distinguish angle 


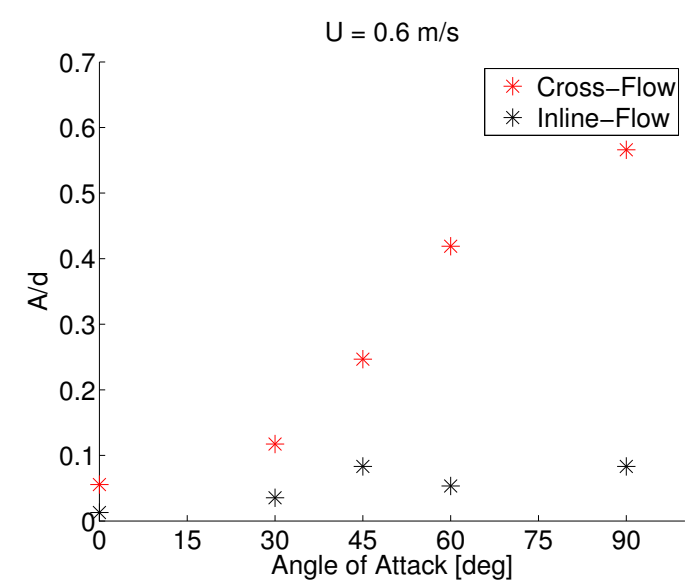

(a)

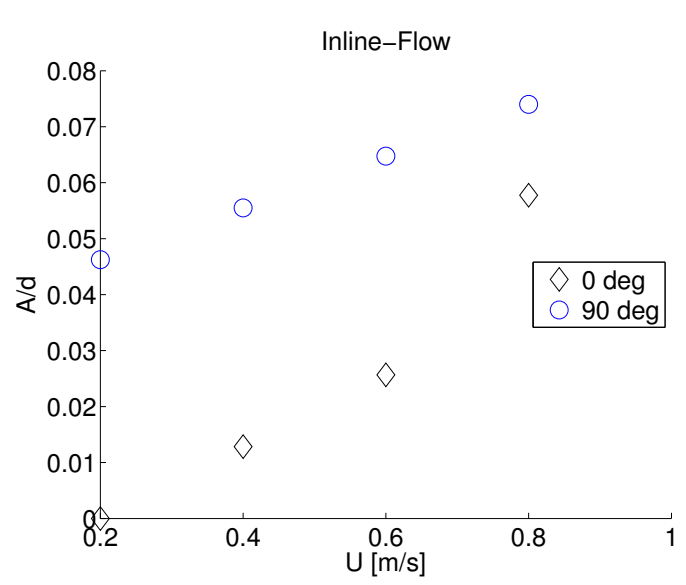

(b)

Figure 3-9: (a) Nondimensional amplitude of inline and crossflow oscillations for the whisker at various angles of attack to the oncoming flow. Each amplitude (A) is divided by the average cross-stream diameter at $\alpha=0^{\circ}: d=1.59 \mathrm{~cm}$. (b) Deflection in the drag (inline) direction vs. velocity (Sensor 3 and Sensor 1 for $\alpha=0^{\circ}$ and $90^{\circ}$, respectively). The next design iteration will include more sensitive measuring devices so that the low-speed, low-angle of attack results can be better distinguished above the inherent system noise.

of attack with $30^{\circ}$ accuracy. Note that the results presented here are not intended to depict a thorough study of the VIV response of the whisker geometry. That study was shown in Chapter 2, in which measurement devices with higher resolution are used and where boundary conditions and mass-damping are carefully considered. Rather, this work presents a device that captures the width of the vibration response range and then utilizes it for heightened sensing.

\subsubsection{Research Cruise Deployment}

To determine the sensor's performance and practicality as an ocean-ready instrument, it was tested at sea. The sensor was deployed from the $R / V$ Wecoma on September 24, 2011 during the UNOLS Chief Scientist Training Cruise near Astoria Canyon, off the coast of Oregon $\left(46.22^{\circ} \mathrm{N}, 125.22^{\circ} \mathrm{W}\right)$. The sensor was mounted to the frame holding the CTD rosette, as shown in Figure 3-10. Both pressure housings were strapped to the outer frame and the whisker segment protruded into the flow, largely unobstructed. The instrument was lowered to a depth of $20 m$ (at 1405 GMT), held 
in place for 10 minutes, and then brought back to the surface (at 1415 GMT).

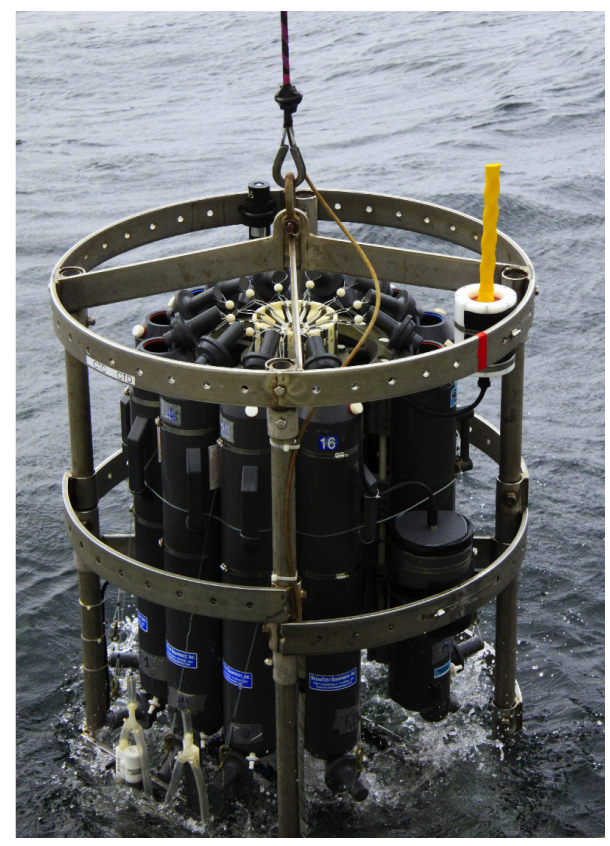

Figure 3-10: Field test setup. The sensor is mounted to the CTD rosette in a manner that allows the whisker to encounter unobstructed flow. The view is from the port side of the ship. (Photo courtesy of Paul Suprenand)

The sensor successfully collected data and maintained the watertight seal. The velocity measurements from the ship-mounted ADCP (RDI 300kHz Ocean Mariner, sampling frequency $=1 / 120 H z)$ are taken as the results to be compared against. That data, shown in Figure 3-11a, was processed through UHDAS CODAS. Data from two of the Bend Sensors during the 10-minute run was sent through a low-pass Butterworth filter and are shown in Figure 3-11b. Sensor 3 shows oscillations and various ramps in deflection. Sensor 2, shows deflection, but oscillations are minimal and are mostly a result of noise. This suggests that Sensor 2 is in the mean flow's inline direction and Sensor 3 in the crossflow direction. Sensor 3 was roughly aligned with the ship's bow, and the ship's heading during the time of deployment ranged from $-120^{\circ}$ to $-140^{\circ}$. The ADCP data: (+) u and (-) v values indicates a southeastwardly flow. This is also what is the whisker sensor data indicates, as Sensor 2 should therefore roughly be in-line with the southeast direction (Figure 3-12).

The amplitude of the in-line deflection can be used to determine the flow velocity. 


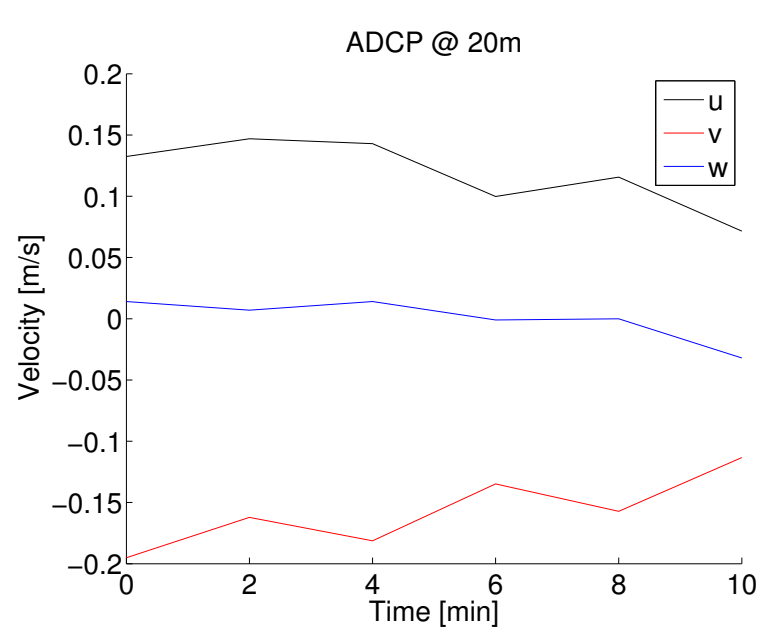

(a)

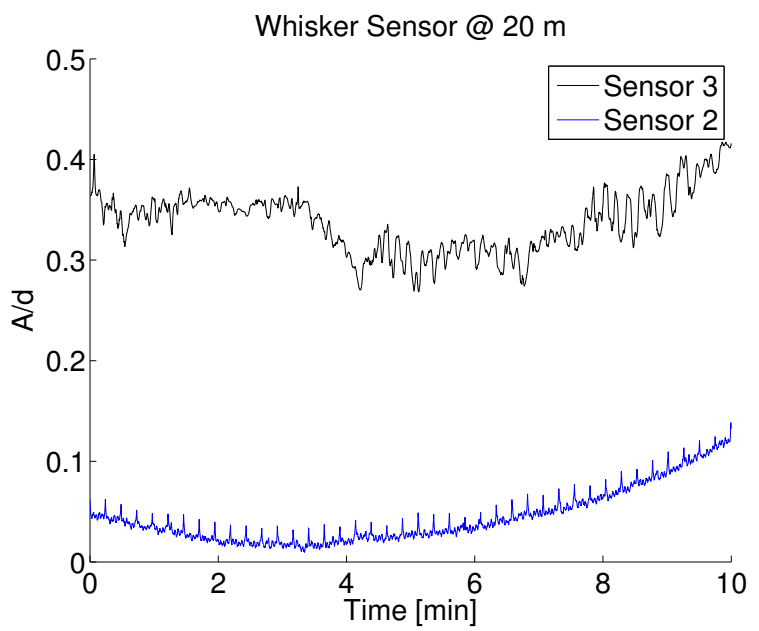

(b)

Figure 3-11: (a) Velocity measurements at 20m depth from the ADCP and (b) voltage output from the whisker-inspired sensor.

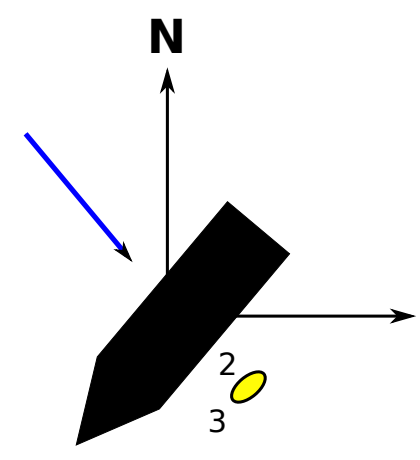

Figure 3-12: Orientation of the ship and sensor. The ship had a heading of $-120^{\circ}$ to $-140^{\circ}$ during the $10 \mathrm{~min}$ deployment. The arrow indicates the general flow direction shown by the ADCP. The numbers indicate the orientation of the corresponding Bend Sensors.

At the beginning of the run, the value is around $A / d=0.05$, which from Figure $3-9 \mathrm{~b}$ indicates $\sim 0.2 \mathrm{~m} / \mathrm{s}$, which matches the same rough value expected from Figure 3-11a. Some drift appears towards the end of the run, however, so this value may be of low accuracy.

Discrepancies between the two sensors can be attributed to a few factors. The motion of the CTD rosette frame, which is hung on a relatively thin cable, does not match the motion of the ship exactly. Also, the flow field being measured was disturbed to some degree by the arrival of the large frame directly preceding the 
measurements. Finally, the whisker sensor does not account for $w$ (vertical) velocity. Future tests should hold the sensor in position longer to allow effects from preceding motions to die out, and they should include a gyro and accelerometers on the sensor/frame to account for any motion of the sensor itself. Nonetheless, these results serve as preliminary evidence that the sensor can be used to detect orientation and velocity in the field. 


\subsection{Flow Sensor: Version 2}

\subsubsection{Design}

A design iteration on the first prototype was implemented in order to 1) increase device performance and 2) enable systematic studying of its flow-induced vibration characteristics. The Bend Sensors used in Version 1 were found to plastically deform after prolonged use. Strain gauges were chosen as a superior alternative by providing low hysteresis and higher resolution. The design was also changed to allow direct measurement of the response in both axes. This version can therefore be employed as a free vibration setup with measurements in both degrees of freedom.

The new design is shown in Figure 3-13. It includes the same 30x-scale plastic whisker model (average crossflow diameter $d=1.59 \mathrm{~cm}$, length $l=27.5 \mathrm{~cm}$ ) mounted in a cantilevered configuration as before. Now the model is connected to a four-armed flexing plate with strain gauges (Omega, Model KFH-03-120). Similar cruciform flexure-based designs have been used in other types of sensors [9, 28, 101].

As the whisker and flow field interact, the four arms bend accordingly, allowing free vibration in both in-line $(x)$ and crossflow $(y)$ directions. A voltage signal is read from the strain gauges, and this is then calibrated to the deflection at the model tip. This system is designed for detailed towing tank tests, rather than use in the field. To this end, it is not designed to be submerged. The strain gauges and flexure remain above the waterline during tests. After the strain gauges are applied to the plate, the entire plate is painted with Rust-Oleum to mitigate rusting.

Figure 3-14(a) depicts the flexure's response to deflection of the whisker. As the rigid model moves in response to the flow, the flexure bends, inducing strain on its arms. One strain gauge is placed on the top side of one arm in each axis. These two measurements can then be used to determine how much the whisker is moving in each respective axis.

A top-down view of the flexure device is provided in Figure 3-14(b). A fourarmed plate of 1095 spring steel (thickness $=0.38 \mathrm{~mm}$ ) was fabricated using a water jet. Holes in the middle allow connection to the base of the whisker model. The 


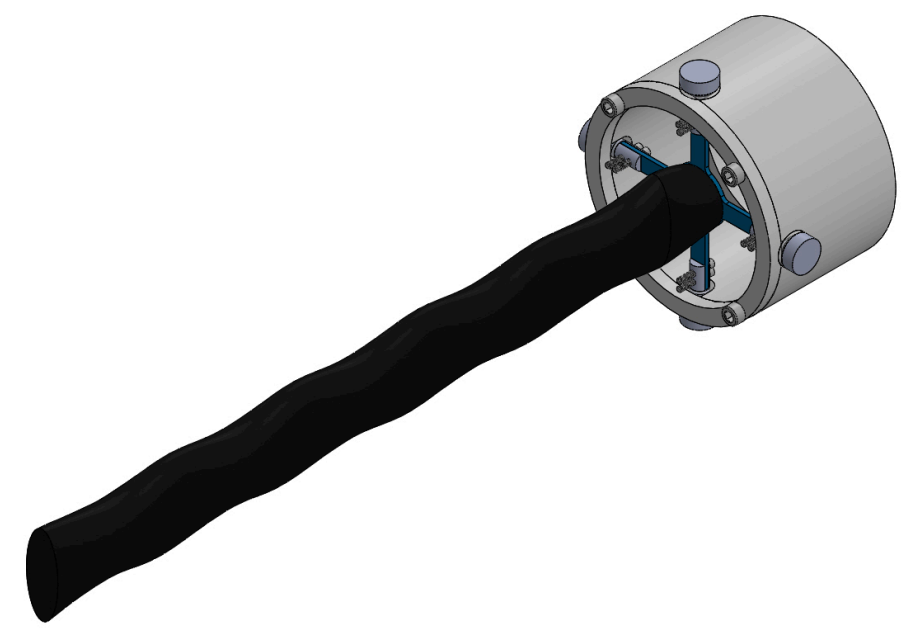

Figure 3-13: Isometric view of the wake sensor, which consists of a plastic whisker model mounted on a four-armed flexing plate. Strain gauges on these arms are mounted on these arms for measurement of the whisker's deflections.

end of each arm is mounted to a plastic pin and bushing. These bushing assemblies are designed to reduce coupling between the $x$ and $y$ axes. As the plates in one axis deflect, the bushings on the off-axis rotate, preventing the off-axis plates from deflecting unnecessarily.

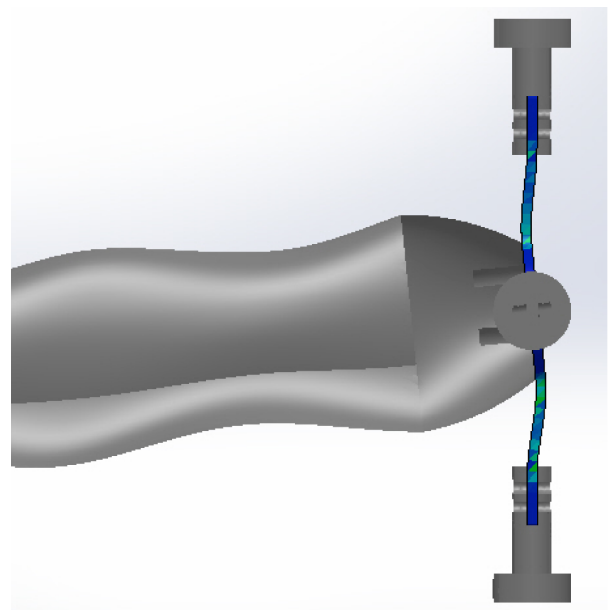

(a)

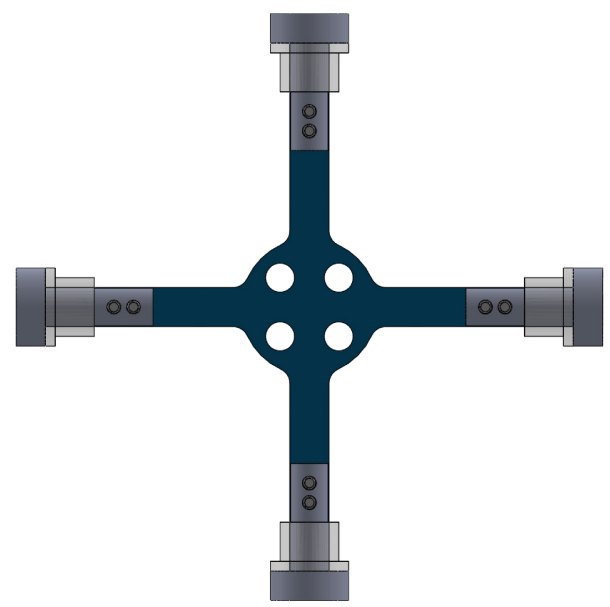

(b)

Figure 3-14: (a) A Solidworks simulation of the strain induced on the flexure as the whisker deflects. (b) Close-up of the flexure device. A spring steel plate made using the water jet is attached to a bushing (clear) and pin (gray) at the end of each arm. This design reduces coupling between the two axes by allowing rotation, rather than deflection in the off-axis. 
The components used for data-collection are shown in Figure 3-15. The strain gauges are arranged in a Wheatstone bridge through the use of resistors soldered to an external board. Their output is acquired with a PhidgetBridge 1046 Input Board (sampling frequency $=60 \mathrm{~Hz}$, gain $=16$ ), which applies basic filtering and sends voltage data to a lab desktop via USB.

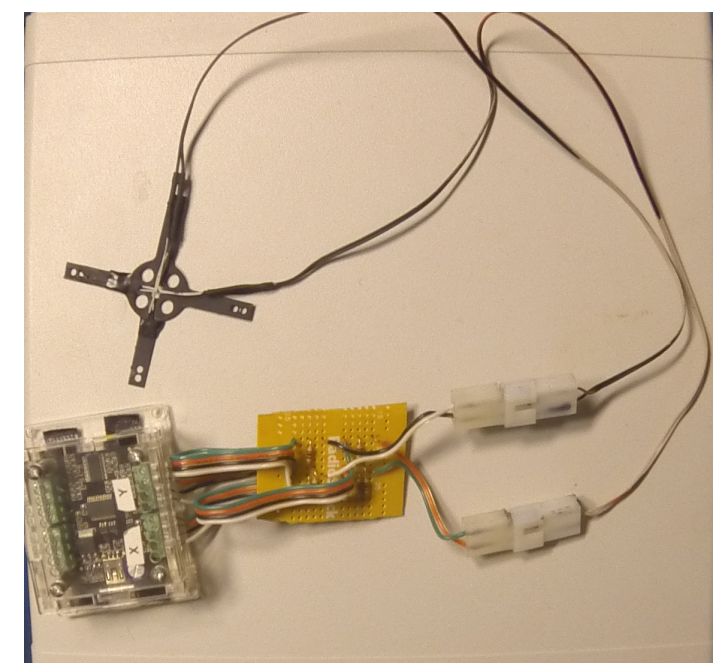

Figure 3-15: The data-collection electronics. The strain gauges are conFigured in a Wheatstone bridge, using additional resistors. A PhidgetBridge is used to filter and transmit the voltages to a computer through USB.

\subsubsection{Calibration}

The flexure's output voltage was calibrated to an equivalent whisker-tip deflection. Bench top measurements were conducted for this calibration. The sensor was held in place in air and deflected in $5 \mathrm{~mm}$ increments. The output voltage $(V)$ at each deflection was recorded and compiled. Figure 3-16 shows the result in the $x$ direction. The calibration curve is taken to be the fit through these points. Both axes were found to have linear calibration curves, with the coefficient of determination $\left(R^{2}\right)$ value being 0.98 and 0.97 in $x$ and $y$ respectively.

The natural frequency $\left(f_{n}\right)$ of the whisker sensor was found by conducting pluck tests and calculating the zero-crossing frequency of the signals. Figure 3-16 shows the time series reading of the pluck test in air for the $y$ direction. In air, the natural fre- 

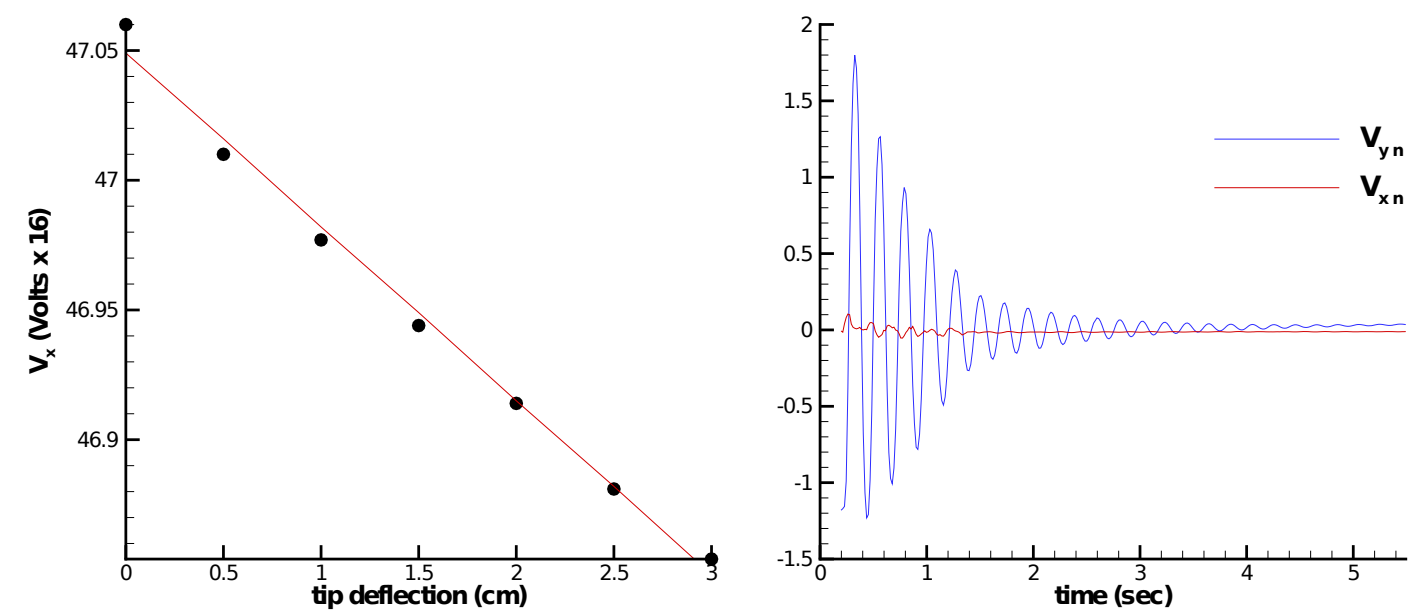

Figure 3-16: (Left) A linear fit was placed through data collected for different tip deflections of the whisker in $x$. (Right) A pluck test in air was used to find the natural frequency of the whisker in $y$. The values here $\left(V_{y n}, V_{x n}\right)$ are centered around zero by subtracting the mean of the signal.

quency in both directions is $f_{n}=5 \mathrm{~Hz}$ in both axes. In water, the natural frequencies are $f_{n x, w}=3 H z$ in-line and $f_{n y, w}=2.2 H z$ in crossflow.

\subsubsection{Coupling}

Excessive friction at the bushings' contact points can lead to a coupled deflection between the $x$ and $y$ axes. In the first fabrication attempt of this design, tolerance in the machining of the pin/bushing assemblies was not tight enough. As the model deflected solely in the $x$ axis, the $y$ axis also bent, and vice versa. Pins with a tighter tolerance were fabricated and found to cause much less friction in the system.

To carefully test whether this coupling was indeed a result of friction, screws were placed through the bushings to completely restrict their rotation. Figure 3-17 shows a picture of the assembly in its normal (low-friction) configuration and then with screws inserted through the bushings.

Pluck tests in water display the increased coupling when the bushings are restricted in rotation. Figure 3-18 shows the power density (normalized by the maximum) for the two configurations. In the normal configuration, an excitation in the $y$ 

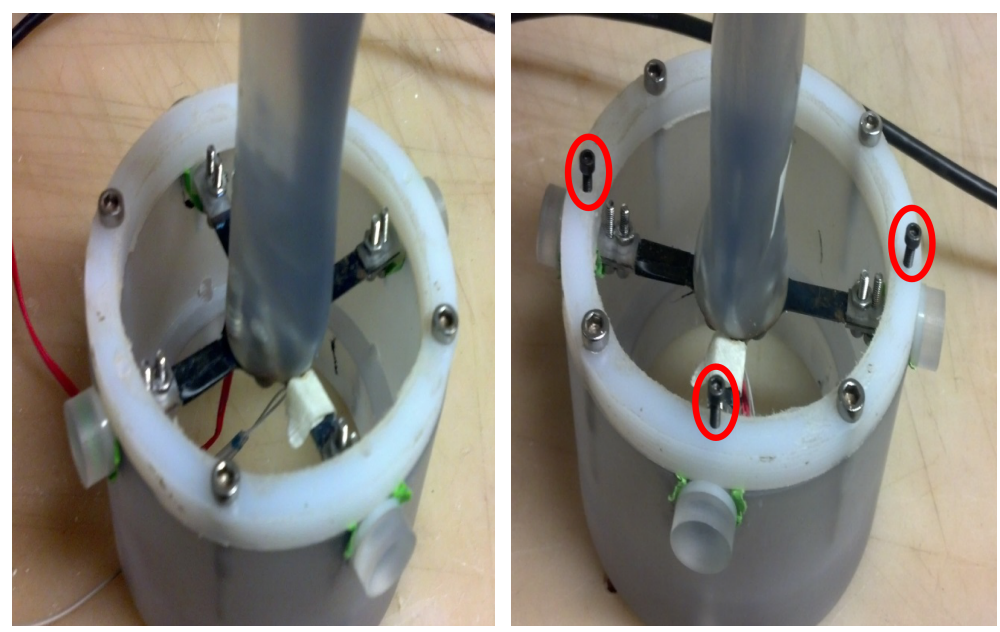

Figure 3-17: Picture of sensor assembly in the (left) normal configuration and (right) with the addition of set screws (circled in red) to restrict bushing rotation. A fourth set screw through the remaining bushing is not visible from this view.

direction causes an oscillatory response almost entirely in that same direction. Minimal response, and therefore minimal power, is seen in the $x$ signal. On the other hand, when screws are placed in the bushings, an excitation purely in the $y$ direction can lead to significant response in both directions. In this case, the power in $x$ is even larger than that in $y$.
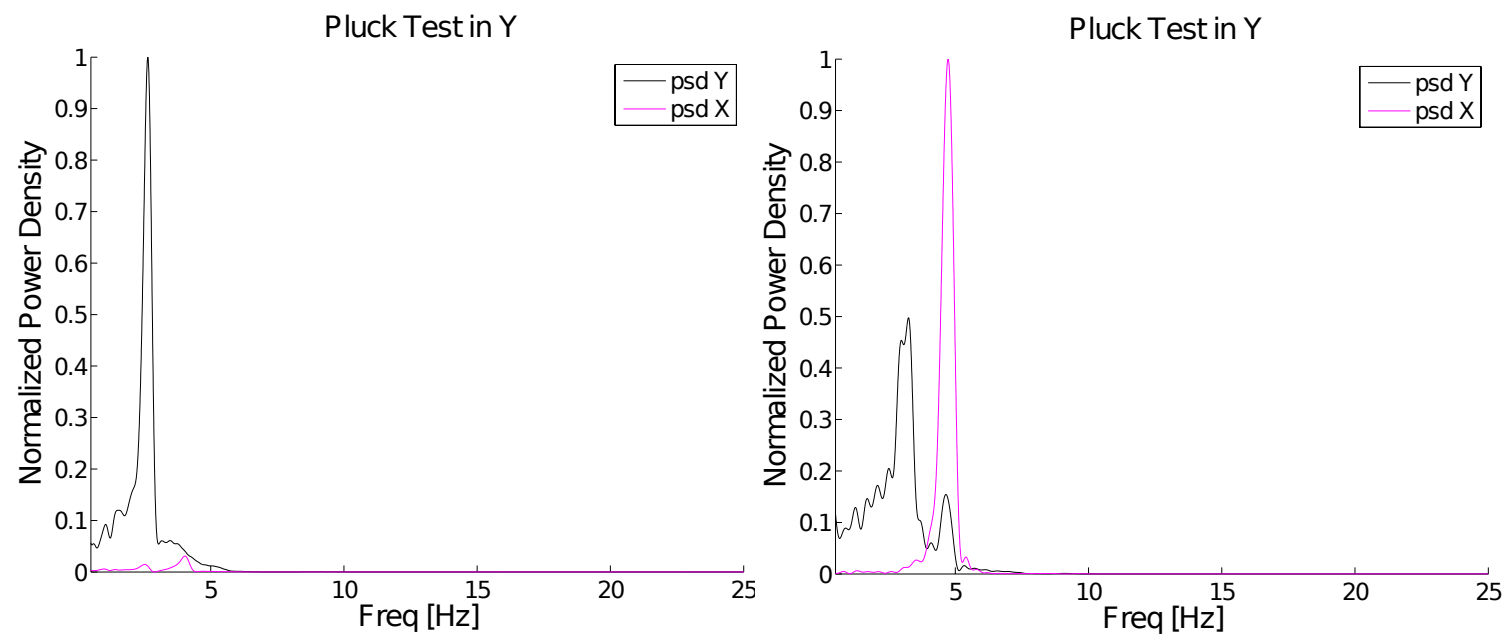

Figure 3-18: Power density of signals from $y$-direction pluck tests in water for the (left) normal configuration and (right) configuration with no bushing rotation.

The VIV characteristics of the sensor in both configurations were measured using towing tank tests. The sensor was mounted vertically above the water line with $70 \%$ 
of the whisker section protruding into the water. The sensor was towed at several reduced velocities $\left(U_{r}=\frac{U}{f_{n y, w d}}\right)$.

The deflection amplitudes in the crossflow direction are plotted in Figure 3-19 for the case when the whisker is towed with no coupling and when it is towed with the set screw configuration that induces coupling.
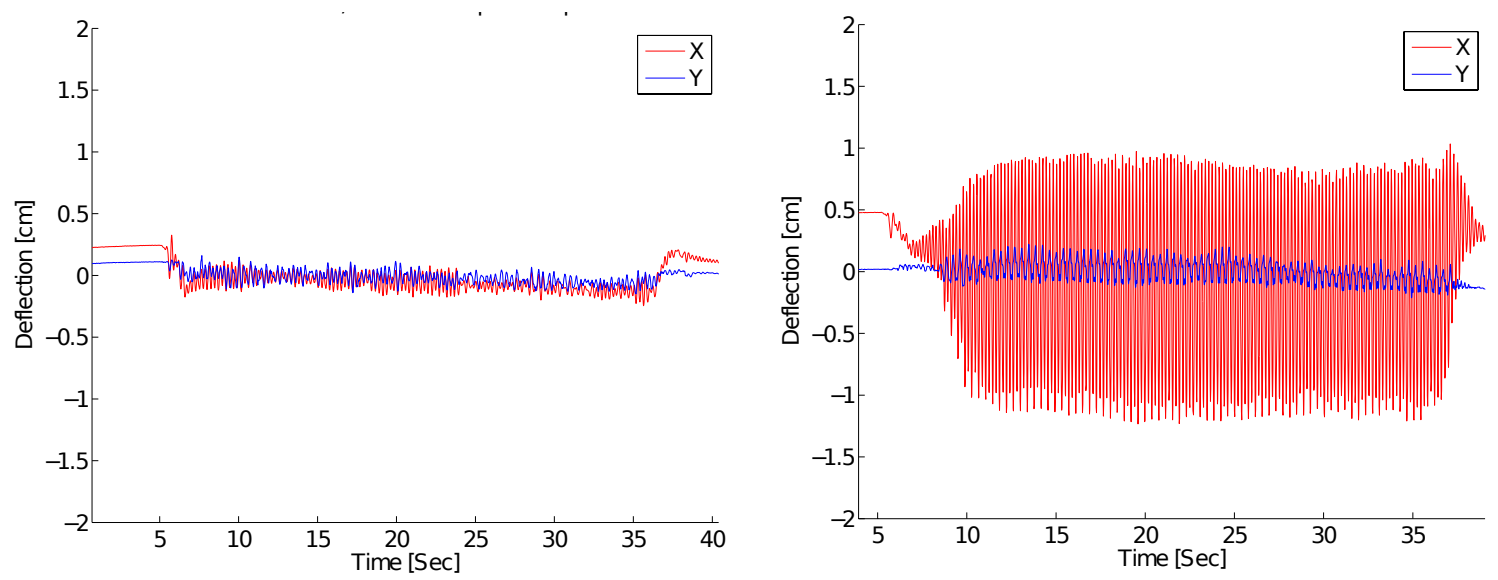

Figure 3-19: Vibrational amplitude in both axes when (left) no set screws were used and (right) set screws were used to restrict bushing rotation. $U_{r}=17$.

\subsection{Conclusion}

Two design iterations of a bio-inspired flow sensor have been designed, fabricated, and characterized. In both cases, the sensing principle is based on deflection measurements at a flexible base, similar to how real seals collect information in their cheek based on the whisker motion. Version 1 serves as a proof-of-concept that the whisker VIV properties can be realized in a sensor. Towing tank tests and field tests demonstrate its ability to operate as a low-cost, low-power flow velocity and direction sensor. Version 2 is created as a platform for systematic studying of flow-induced vibration properties, with the goal of developing wake detection capabilities. 


\subsubsection{Version 1}

The first version shows the sensor's ability to use drag-based measurements to determine flow velocity. Readings from Bend Sensors connected to the base of the whisker are calibrated to provide flow velocity based on the deflection in the drag direction. The sensor can be tuned to operate optimally for a desired range of flow speeds, simply by replacing the silicone membrane with one of a different rigidity. Also, it captures a wide range of vibrational response with flow angle, as predicted in Chapter 2. The Bend Sensor readings can therefore also be used to detect the direction of oncoming flow.

The sensor was designed such that flexible motion is possible even while a watertight seal is maintained. Field tests confirm that the device can be used at depth. This indicates the sensor's ability to be used for measuring currents along the water

column or while mounted on an AUV. The data logging is done automatically once the system is activated, allowing this sensor to be used in a stand-alone configuration.

This device presents itself as a low-power alternative to standard velocity sensors, such as DVLs, as it does not require active emission of signals in order to conduct detection. Also, those devices may cost tens of thousands of dollars, whereas this costs on the order of hundreds of dollars or less.

\subsubsection{Version 2}

The second version was designed with the goal of creating a system that can conduct vortex-wake detection. Existing sensors either cannot conduct passive detection or else can not do so without experiencing significant background "noise" while moving at high speeds. A sensor designed equivalently, but with a circular rod in place of the harbor seal whisker geometry, would have difficulty distinguishing self-induced vibrations from the vibrations induced by oncoming wake features.

This device was therefore designed to again capture the VIV reduction properties of the whisker geometry and now to also allow for control of important parameters for wake-induced vibration studies (i.e. mass-damping and natural frequencies). The 
improved design is a flexure-based system with strain gauges. Simply changing the thickness of the flexure allows for variation of the aforementioned properties.

Importantly, contributions from the in-line and crossflow directions of motion are now separated. The two axes are still physically connected, however, which can lead to coupling if excessive friction is present. That coupling can lead to a dramatic increase in the VIV response. The reason for this may be that the coupling attempts to induce a torsional motion, which is what was observed in a separate experiment conducted with a flexible whisker model (Appendix C). A torsional response may be a result of the elliptical cross section [137.

This new system doubles as a free vibration experiment setup, on which detailed wake-induced vibration studies are conducted in Chapter 4. Those experiments are used to study and develop the wake detection capabilities of the sensor.

\subsubsection{Future Work}

Room for improvement remains in the design, particularly in terms of size reduction and increased sensitivity. The designs presented here are intended to serve as the groundwork for what can be done with seal-whiskerlike sensors. Future integration into an AUV sensor suite will require miniaturization of the device and a waterproof design. Further testing of alternative sensing devices is also merited. Optical fibers, for example, could prove superior to strain gauges in this system.

For future work with this device as a velocity and direction sensor (as in Version 1), it is recommended that a row of whiskers be used. Each whisker should be oriented at a different angle to the oncoming flow, allowing for accurate quantification of the velocity and direction.

For future work with this device as a wake sensor, please see Chapter 4. The goal is for this device to enable targeted sensing for AUVs. The ability to detect and track a vortex wake would open doors for the oceanographic community to use robotic systems to home in on hydrothermal vents or enable long-term behavioral studies by following animal schools for extended periods of time. 


\section{Chapter 4}

\section{Whisker in Vortex Wake: Free}

\section{Vibrations}

What is the seals' mechanism of wake detection?

\subsection{Motivation}

In [58] and in Chapter 2 of this thesis, flow simulations showed that the whisker geometry's undulations break the spatial coherence of vortex formation and form streamwise vorticity, hence reducing substantially the unsteady fluid forces on the whisker. This result implies that the seal can move forward at steady speed with small self-induced noise from VIV. The question remains on how the vibrissae detect unsteady velocities, especially the minute velocities found in wakes $30 \mathrm{sec}$ after a body has passed [138].

To answer this question we turn to the problem of wake-induced vibrations (WIV), which occur when a flexibly mounted body is placed in the wake of an upstream body so it is subject to the unsteady forcing of its vortical structures. Most research has been conducted in studying two interacting circular cylinders of equal or comparable diameter [65, 102, 5, 4] and it is shown that the upstream cylinder has significant effect on the amplitude and frequency of motion of the downstream cylinder even when it is 
placed at 25 diameters apart. It was also found that the vortical wake of the upstream cylinder causes a restoring force on the downstream cylinder, which is equivalent to a linear spring, hence affecting its natural frequency [3]. In the few studies conducted with interfering cylinders of unequal diameter [115, 82, 40, 41, 39], it was found that the upstream cylinder also affects the response of the downstream cylinder, reducing its amplitude relative to the single cylinder case, while the frequency of oscillation of the downstream cylinder was found to be either equal to, or twice the value of the frequency of the upstream cylinder. Only one published study [91] has begun to show the harbor seal whiskers' response under WIV conditions.

In this chapter we study a flexibly mounted model of a seal whisker placed within the wake of a larger upstream circular cylinder. Strain measurements and dye visualizations are used to elucidate the whisker's mechanism of detecting the features of the unsteady flow. The effects of the separation distance and size difference between the two objects are studied. Experiments where the whisker model is replaced first by a circular cylinder and then by an elliptical cylinder are also conducted to compare their responses.

\subsection{Methodologies}

The whisker model was mounted in a vertical position in the MIT Towing Tank, fixed at its top on a spring-like structure, which allowed it to vibrate in crossflow and in-line vibrations, as explained in detail in the next section. The models' VIV responses were first tested by towing them in calm water. Next, a hydrodynamic wake was generated upstream of the whisker model through the use of a vertically mounted cylinder, which sheds a von Kármán vortex street (Figure 4-1).

In the case of the live animal, the whisker diameter, which is typically about 0.5 to $1 \mathrm{~mm}$, is expected to be significantly smaller than the dimension of the dominant wake vortices, which scale with the dimension of the upstream body. The diameter of the upstream cylinder was, therefore, chosen to be larger than that of the whisker model $\left(d_{c y l}=\{2.5,4,11\} d_{w}\right)$. The two objects were towed simultaneously along the 


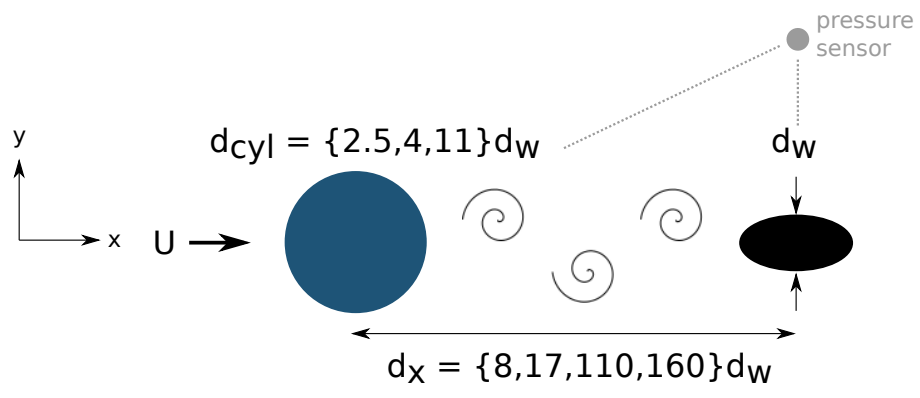

top view

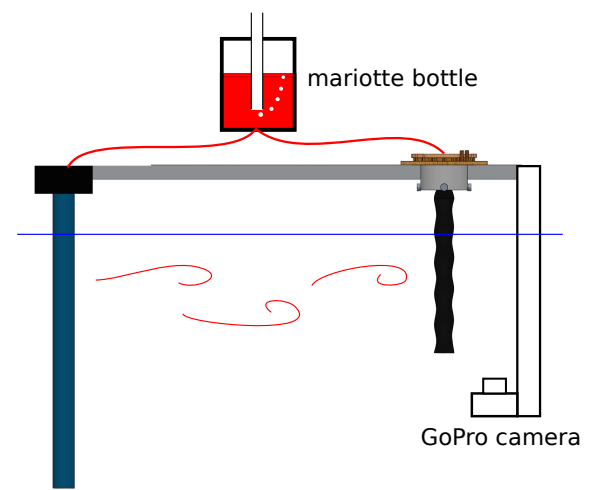

side view

Figure 4-1: (Left) Top view; and (Right) side view of the experimental setup. A hydrodynamic trail was generated in front of the whisker through the use of a cylinder, which shed a Karman vortex street. Three different downstream distances $\left(d_{x}\right)$ were tested for each of the three models, ranging from the near wake to the far wake. Three different cylinder sizes $\left(d_{c y l}\right)$ were also tested. Simultaneous measurement of the oncoming wake frequency and whisker response was done through use of a pressure sensor placed near the edge of the wake. A mariotte bottle supplied dye at a constant flow rate. A camera underneath the model captured a bottom-up view of the wake-whisker interaction.

tank at velocity $U$, and the whisker's amplitude and frequency of vibration were measured. The value of $U$ covered a range from 0.1 to $1.3 \mathrm{~m} / \mathrm{s}$.

To assess the effect of the distance between the two objects, the tests were conducted at different downstream distances $\left(d_{x}=\{8,17,110,160\} d_{w}\right)$, representing cases of close proximity, moderately large distance, and large distance. These tests were repeated with the whisker model replaced by a smooth elliptical (2:1 diameter ratio) cylinder model and then a circular cylinder model.

\subsubsection{Note on the geometry}

Two versions of the whisker geometry are tested in the present study. The geometry of the first whisker was taken directly from that provided in [58] (as in Chapters 2 and 3) and scaled up 30x. It is referred to here as "whisker \#1" and it has an average diameter in the crossflow direction $d_{w}=1.59 \mathrm{~cm}$. A second whisker geometry was designed ("whisker \#2"). It more closely matches the shape measured on real 


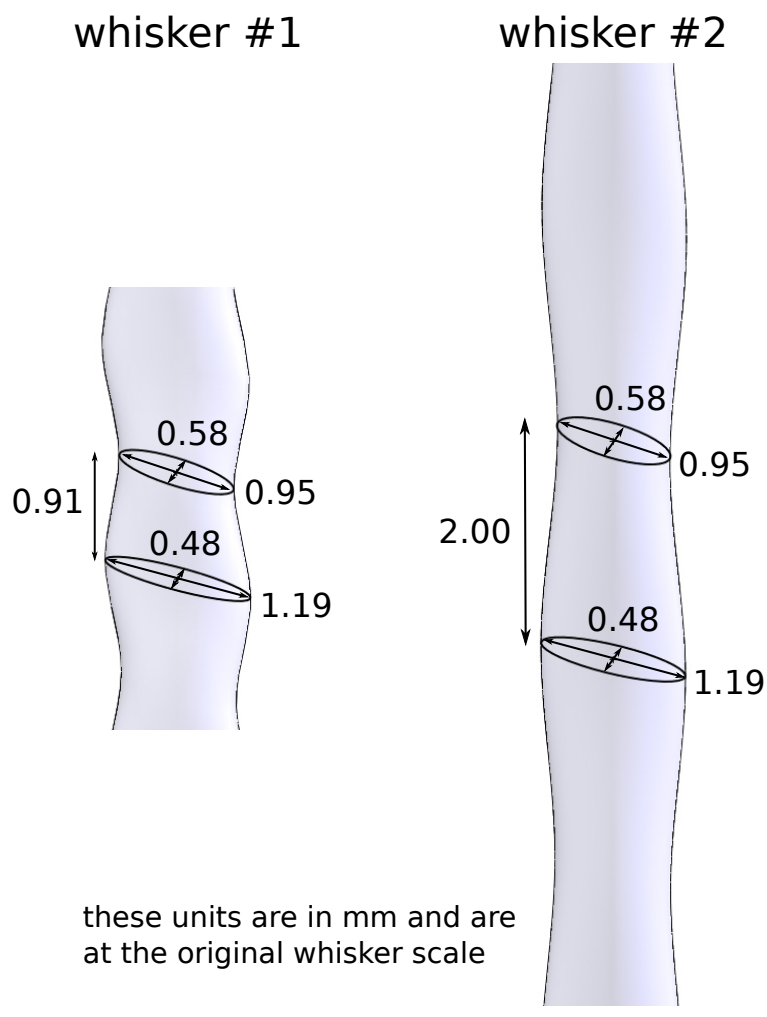

Figure 4-2: Two variations in the harbor seal whisker geometry are captured through the depicted parameters, which are shown here at the scale of the real seal. The inclination angles are $17.6^{\circ}$ and $15.27^{\circ}$ for the top and bottom cross-sections shown, respectively. The geometry from Hanke et al (2010) ("whisker \#1") has a $\lambda / D_{m} \approx 2$, whereas the new geometry ("whisker $\# 2$ ") has a $\lambda / D_{m} \approx 5$. The models used in this chapter are scaled up from the dimensions shown here 30 and 20 times, respectively.

harbor seal whisker specimens both that the authors received from the New England Aquarium and as described in [45]. This model was enlarged 20x from the real whisker scale, providing it a diameter $d_{w}=1.06 \mathrm{~cm}$. The only nondimensional difference between the two geometries is the wavelength-to-diameter ratio: $\lambda / D_{m} \approx 2$ in the first and $\lambda / D_{m} \approx 5$ in the second, where $D_{m}$ is the mean overall diameter.

\subsubsection{Strain Measurements: Free Vibrations}

\section{Apparatus (Flow Sensor Version 2)}

As detailed in Chapter 3, a whisker sensor was designed to freely vibrate by mounting the base of the model on a flexing plate $(1095$ spring steel, thickness $=0.38 \mathrm{~mm}$ ) with four arms, which allows the model to freely vibrate in the in-line $(x)$ and crossflow $(y)$ 


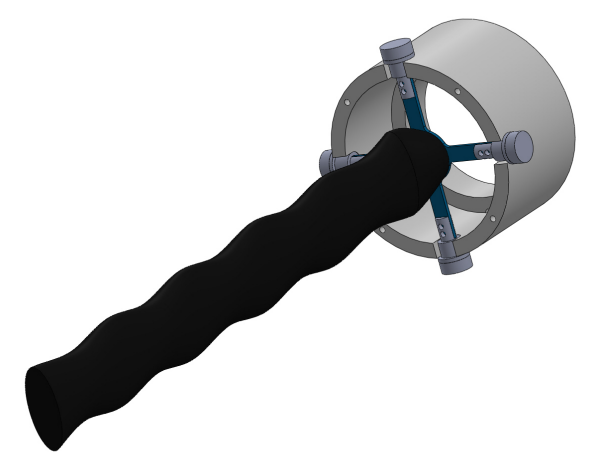

Figure 4-3: The model whisker ("whisker \#1" pictured here) was mounted on a set of flexing plates that allow free vibration in in-line and crossflow directions. Bushings are used to reduce coupling between the axes.

directions (Figure 4-3). It was therefore in a cantilevered configuration, resembling that on the seal, although the main body of the whisker remains rigid in the experiment. This same device is used throughout this chapter to conduct free vibration experiments.

Strain gauges (Omega, Model KFH-03-120) on the flexing plates were arranged in a Wheatstone bridge to output a signal proportional to the deflection of the plates. Strain signals were converted to a voltage once acquired through a PhidgetBridge 1046 USB Input Board (sampling frequency $=60 \mathrm{~Hz}$, gain $=16$ ). The measurements collected were calibrated to the amount of deflection at the tip of the model. Calibration curves relating voltage and tip deflection were found to be linear for both axes on all models, with the lowest coefficient of determination $\left(R^{2}\right)$ value being 0.953 .

The natural frequency $\left(f_{n}\right)$ of the models was found by conducting pluck tests in water and calculating the zero-crossing frequency of the signals. The whisker \#1 and elliptical cylinder models have a natural frequency of $f_{n, x}=3.1 \mathrm{~Hz}$ in-line and $f_{n, y}=2.2 \mathrm{~Hz}$ in crossflow. The whisker \#2 model was designed to have similar mass to these two, allowing it to have similar natural frequencies $\left(f_{n, x}=3 \mathrm{~Hz}, f_{n, y}=1.8 \mathrm{~Hz}\right)$. Keeping the mass of the two whisker models similar (for ease of experimental setup) meant that they needed to be at different scales in order to maintain the same number of undulations on each model. This is the reason that the whisker \#1 model was scaled up 30 times whereas the whisker \#2 model was scaled up 20 times. The circular 
cylinder model has a smaller mass relative to the other models, giving it a lower frequency $\left(f_{n}=1.8 \mathrm{~Hz}\right)$, which also necessitated the use of a slightly thinner flexure (1095 spring steel, thickness $=0.2 \mathrm{~mm})$ to cover an appropriate reduced velocity $\left(U^{*}=U /\left(f_{n} d_{w}\right)\right)$ range.

All four models have a mass ratio $\left(m^{*}=m / m_{d}\right)$ of 1.4 , where $m$ is the model mass and $m_{d}=\rho \pi \frac{d_{w}^{2}}{4}$ span is the displaced fluid mass. The whisker \#2 model has a submerged span of $L / d_{w}=30$ and the other three models have $L / d_{w}=12$. The Reynolds number based on the diameter, $R e=U d_{w} / \nu$, ranges from 1,060 to 20,670, so it is generally larger than the Reynolds number of seal whiskers which is around 1,000. The higher Reynolds number in the experiments allows easier measurement of forces and motions.

\subsubsection{Dye Visualizations: Free Vibrations}

\section{Apparatus}

A mariotte bottle setup was constructed in order to introduce dye into the flow for visualizations. This system is made of a sealed container of dye with an air inlet on the top and a dye outlet on the bottom (Figure 4-1). The air inlet ensures that the pressure inside remains constant even as the reservoir level lowers. The dye can therefore be delivered at a constant rate. The tracer was made by combining 8 parts water, 5 parts industrial food coloring, and 1 part alcohol, which results in a neutrally buoyant liquid.

The dye was introduced into the flow between the wake generator and the whisker. A tube from the mariotte bottle extends into the wake generator (hollow PVC pipe) and dye exits from a small hole on the downstream side of the cylinder. The hole aligns with the $65 \%$ span level of the whisker. An underwater camera captured videos of the wake-whisker interaction. A GoPro Hero 3 Black Edition was mounted below the whisker, which provided a bottom-up view. It was positioned sufficiently below and downstream of the model to avoid disturbing the flow seen by the model. Videos of the models' wakes were captured with 720p resolution and at 120 frames per second. 
Videos of the cylinder wake-whisker interaction were captured at 1440p resolution and at 48 frames per second.

\subsubsection{Wake Frequency Measurements}

Finally, to compare the whisker's response to the specific wake being generated, information on the upstream wake was measured in real time during the towing tests. A pressure sensor (Freescale MPXV7007, on-chip conditioning) was placed at the same downstream location as the whisker (Figure 4-1). To avoid interfering with the flow encountered by the whisker, the pressure sensor was placed at a lateral location closer to the edge of the wake (at an angle of $\beta=60-75^{\circ}$ between the upstream cylinder and the whisker). Plastic tubing was connected to the sensor inlet and placed into the wake, such that the sensor avoided direct contact with water. To reduce flow-induced vibrations of the tube itself, it was attached to the leading edge of a small airfoil wing, which was rigidly mounted to the carriage. A National Instruments USB-6211DAQ board was used to collect the analog signal (sampling frequency $=1000 \mathrm{~Hz}$ ). The vortex formation frequency of the wake was extracted from the pressure signal.

\subsubsection{Analysis}

The strain signals were recorded as function of time for each run. A bandpass Butterworth filter (pass range: 0.4 to $20 \mathrm{~Hz}$ ) was applied. To exclude transient phenomena, only a middle segment of these signals was considered further. The vibration amplitude for that run was determined by taking the root-mean-square (RMS) amplitude of that segment. The value was then nondimensionalized by $d_{w}$. The crossflow frequency of vibration was determined by taking a fast Fourier transform (FFT) of the signal, selecting the peak response frequency, and nondimensionalizing it by the model's natural frequency in water: $f_{y}^{*}=f_{y} / f_{n, y}$.

For the experiments which utilize simultaneous measurements of wake pressure and strain on the whisker sensor, the response was analyzed over a more fine time scale. First, the two signals were re-sampled to the same frequency and the following 
signal conditioning was applied to both signals. A bandpass Butterworth filter was used to pass frequencies between $1 / 2$ and $2 x$ the expected wake frequency, based on equation 1.2. Additional Butterworth filters were used to notch out the natural frequencies of the foil $(\approx 1,10,20 \mathrm{~Hz})$. Cross-correlation of the two signals was conducted to quantify any similarity.

This correlation was first conducted by dividing the signals by their RMS value in order to normalize their magnitudes. An initial cross-correlation was carried out to determine the value of any time delay between the two signals. The signals were shifted by a delay corresponding to the peak in this initial cross-correlation result. A second cross-correlation was then used to determine the level of synchronization between the two signals. This value was normalized by the magnitude to reach a cross-correlation coefficient $(c)$, which is the variable presented in the following plots.

\subsection{Results}

\subsubsection{VIV response}

First, the vortex-induced vibrations of each model were measured as the models were towed through calm water. The whiskers and elliptical cylinder were oriented with their long axis aligned with the flow direction. Figure 4-4 shows the nondimensional tip deflection for all three models in the crossflow direction $\left(A_{y}^{*}=A_{y} / d_{w}\right)$ and in the in-line direction $\left(A_{x}^{*}=A_{x} / d_{w}\right)$ versus the nondimensional towing velocity $\left(U^{*}=\right.$ $\left.U /\left(f_{n, y} d_{w}\right)\right)$. The nondimensional frequency of crossflow oscillation $\left(f_{y}^{*}=f_{y} / f_{n, y}\right)$ is also plotted.

[37] tested a cylinder in a similar configuration as in our experiments, viz. pivoted at the top, freely vibrating in the in-line and crossflow directions and found a peak amplitude of vibration of $1.5 d$ at the tip. That value is close to what is seen for the cylinder here, providing some validation for this new setup.

The same trends seen in forced vibration experiments (Chapter 2) are found here: The whisker at $0^{\circ}$ experiences low VIV compared to a circular cylinder. The oscil- 

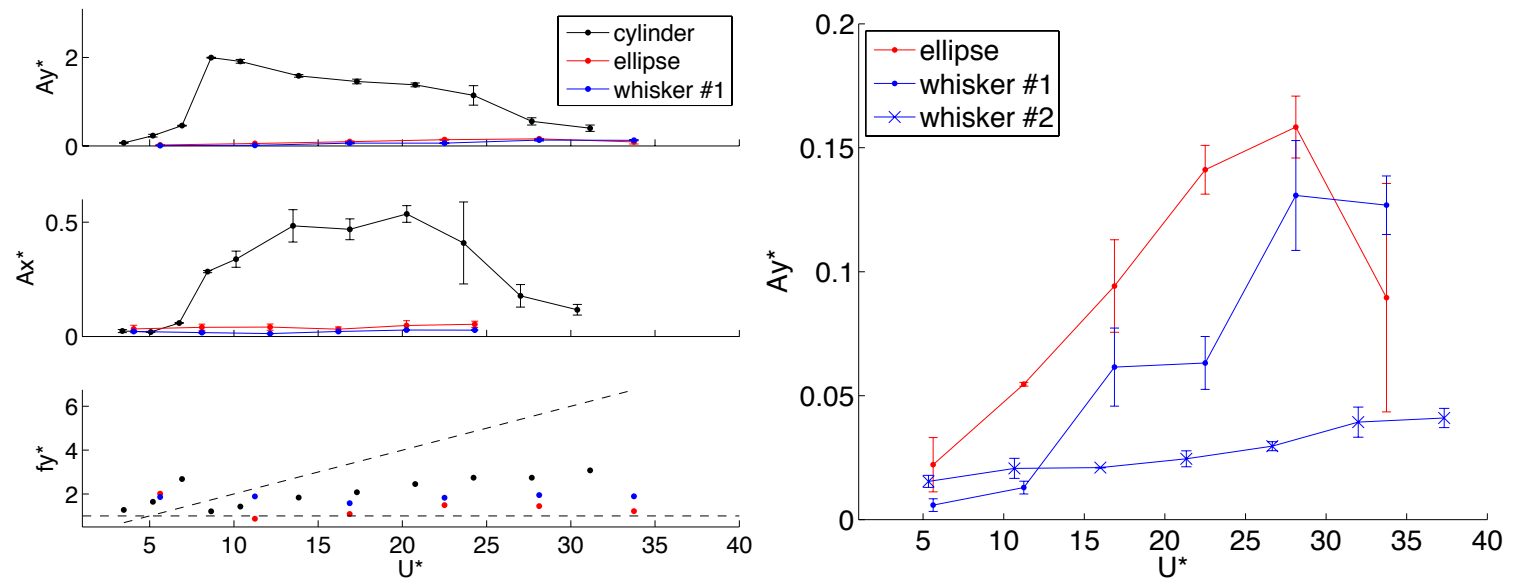

Figure 4-4: VIV response of each model positioned in the streamlined direction $(\alpha=$ $0^{\circ}$ ). Bars depict data spread over three repeat runs. (Left) Both cross-flow and in-line amplitudes of the whisker and elliptical cylinder are significantly smaller than those of the circular cylinder. The frequencies at which the whisker and ellipse vibrate remain close to their natural frequency. The diagonal dotted line indicates a frequency that matches the Strouhal relation. (Right) Both whiskers and the elliptical cylinder vibrate with $A_{y}^{*}<0.2$. Whisker \#2 has the lowest VIV amplitude.

lation frequency for the elliptical cylinder and whisker remain close to their natural frequency across all speeds tested. It should be noted that in previous seal whisker vibration studies ([58] and Chapter 22), the additional degree-of-freedom in the in-line direction was not included. Motion in the in-line axis can induce additional vibrations [17. In spite of this, the whisker's VIV is seen to remain low.

Figure 4-4(b) shows a zoomed in view of the cross-flow response. The elliptical cylinder exhibits similar, slightly higher vibrations than the whisker. The whisker \#2 model has an even lower response than the original model. These two geometry variations may align with the two minimums in response seen in the Lam \& Lin (2009).

Dye visualizations of the $0^{\circ}$ configuration illuminate wake patterns that explain the recorded deflection measurements (Figure 4-5). A circular cylinder sheds large coherent vortices in a $2 \mathrm{~S}$ pattern. The elliptical cylinder also sheds coherent vortices but with a narrower wake. The whisker \#1 model displays a less coherent vortex wake that forms farther downstream. The whisker \#2 model creates a similar wake (Figure 4-6). 

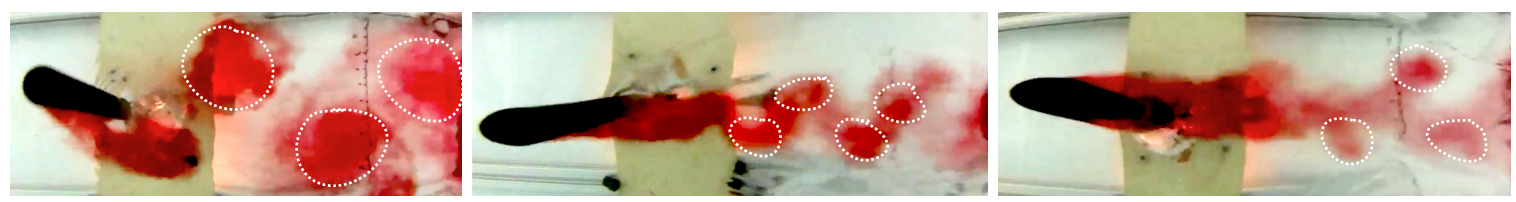

Figure 4-5: Dye visualizations of the wake behind a circular cylinder, elliptical cylinder, and whisker (from left to right) at $\alpha=0^{\circ}$. The first two objects shed regular $2 \mathrm{~S}$ patterns, whereas the whisker \#1 model sheds an incoherent wake. Images were taken during runs corresponding to each model's peak amplitude: cylinder at $U^{*}=10$, ellipse at $U^{*}=28$, and whisker $\# 1$ at $U^{*}=28$.

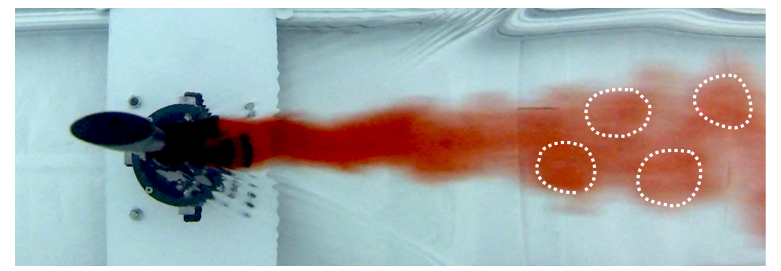

Figure 4-6: Dye visualization of the wake behind the whisker \#2 model. Similar to the whisker \#1 model, it sheds an incoherent wake. The image was taken during the run corresponding to the model's peak amplitude, which is at $U^{*}=32$.

Because of the slightly superior VIV reduction performance of the second whisker model, the remainder of this paper focuses on it. If the model number is not explicitly mentioned, it should be taken to refer to the whisker \#2 model.

\subsubsection{Whisker response within a wake $\left(d_{c y l}=2.5 d_{w}, d_{x}=8 d_{w}\right)$}

We then consider the case where the whisker is at a relatively close distance $\left(d_{x}=8 d_{w}\right)$ from the upstream cylinder $\left(d_{c y l}=2.5 d_{w}\right)$. The whisker is facing the flow in its streamlined direction $\left(\alpha=0^{\circ}\right)$. The whisker's nondimensional deflection at the tip is shown in Figure 4-7(a). The amplitude of vibration in this case peaks at $>1.5 d_{w}$. This value has to be contrasted with the whisker's vibration amplitude as it moves through open water (depicted with the "x"'s), which is significantly lower than that - peaking at $\sim 0.05 d_{w}$. Hence, for this distance from $\left(d_{x}\right)$ and size ratio with the upstream cylinder $\left(d_{c y l} / d_{w}\right)$, the whisker can vibrate with 30x larger amplitude when it encounters the wake than it does in open water. As above, the symbols mark the average of three repeat runs and the bars denote the data spread.

Also of note is the comparison of the whisker's frequency of response between the 
two cases, shown in Figure 4-7(b), where the nondimensional frequency of vibration $\left(f_{y}^{*}\right)$ is plotted versus $U^{*}$. The frequencies plotted here were determined by selecting the peak in the FFT of the corresponding signal's time trace. As the whisker moves through open water, it oscillates at a relatively constant frequency. However, when the whisker encounters the oncoming vortical wake, it oscillates at the vortex shedding frequency of the upstream cylinder $\left(f_{v}\right)$. This frequency is measured with a pressure sensor during the same runs, nondimensionalized by $f_{n, y}$, and plotted with the red bars (again showing the data spread over three runs). Hence the whisker synchronizes with the oncoming wake dominant frequency, which is distinct from its VIV frequency in open water.

These are two strong indicators for wake detection that the seal can potentially use: The whiskers which normally vibrate at low amplitude when in open water, vibrate with more than an order-of-magnitude larger amplitude when in the wake of a body; more importantly, the frequency of vibration synchronizes with the dominant frequency in the wake.

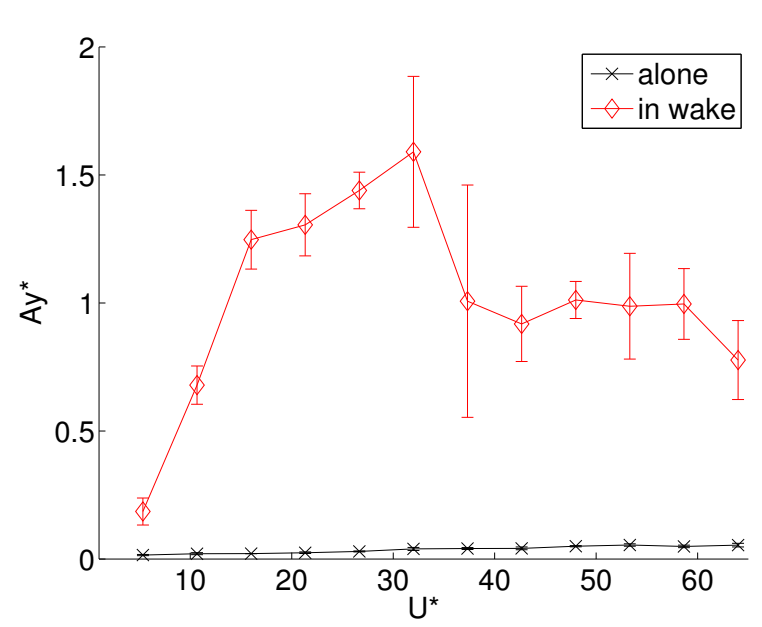

(a)

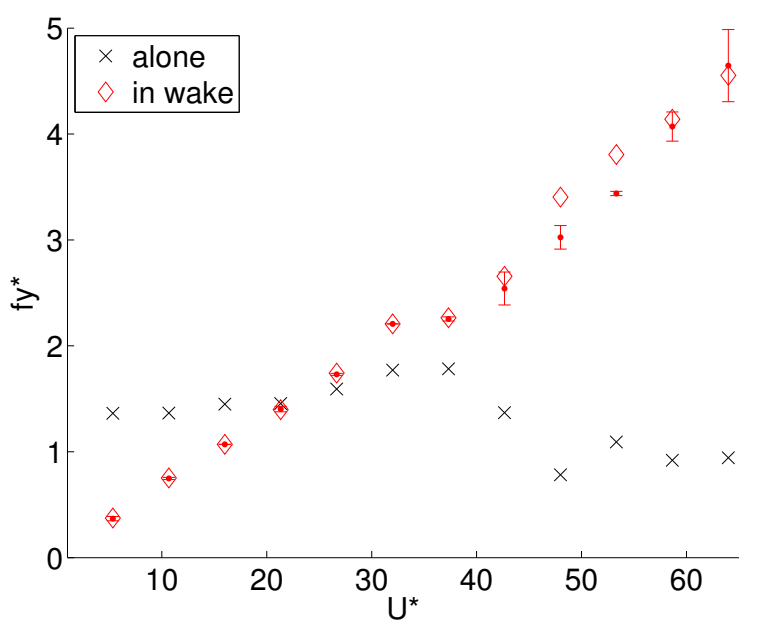

(b)

Figure 4-7: For a whisker within the wake of an upstream cylinder $\left(d_{c y l}=2.5 d_{w}\right)$ at a distance $d_{x}=8 d_{w}$ : (a) The whisker vibrates with far higher amplitude in the wake than it does on its own. (b) The peak frequency of the whisker's vibration synchronizes with the oncoming wake frequency. The diamonds and " $\mathrm{x}$ "s are the average over three repeat runs. The bars indicate the data spread of the measured wake frequency over the same three runs. 


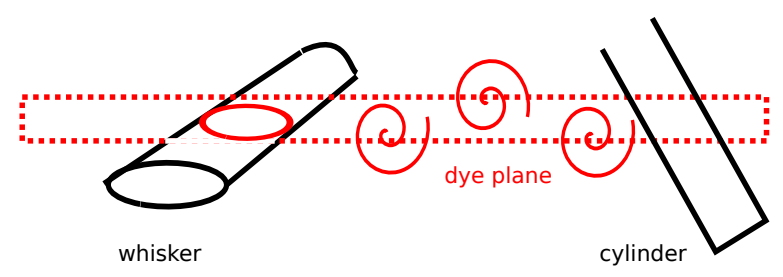

Figure 4-8: Schematic of the camera perspective of the dye runs. The bottom-up view shows vortices being shed by the wake generator on the right and impinging on a cross-section of the whisker on the left.

\subsubsection{Dye visualizations in wake}

Flow visualizations of the whisker interacting with the wake confirms and explains these two findings. Similar parameters displayed above are used here: $d_{c y l}=2.5 d_{w}, d_{x}=$ $17 d_{w}$. Videos are taken with a bottom-up perspective depicted in Figure 4-8, Dye exits the wake generator, off the right side of images, is entrained by the vortices shed by the cylinder and then impinges on the whisker. The whisker is painted white, and the cross-section which aligns with the dye plane is outlined. A sequence of snapshots from the video are shown in Figure 4-9.

The motion of the whisker is driven by the alternating pressure field of the wake, forcing it to oscillate at the wake frequency. It is remarkable that the trajectory of the whisker resembles the slaloming motion recorded for trout swimming behind a circular cylinder, which extracts energy from the oncoming von Kármán vortices by also synchronizing with the frequency of the vortices [85].

\subsubsection{Cross-correlation analysis}

More detailed analysis of the response between the whisker and the upstream wake was conducted by cross-correlating the strain signal at the whisker base with the pressure signal in the wake. A cross-correlation value of 1 indicates perfect synchronization between the whisker and the wake frequencies, and a value of 0 indicates no synchronization.

Figure 4-10 shows a sample result from this analysis. On top are the two signals measured over the course of one run. The signals were normalized and aligned in 

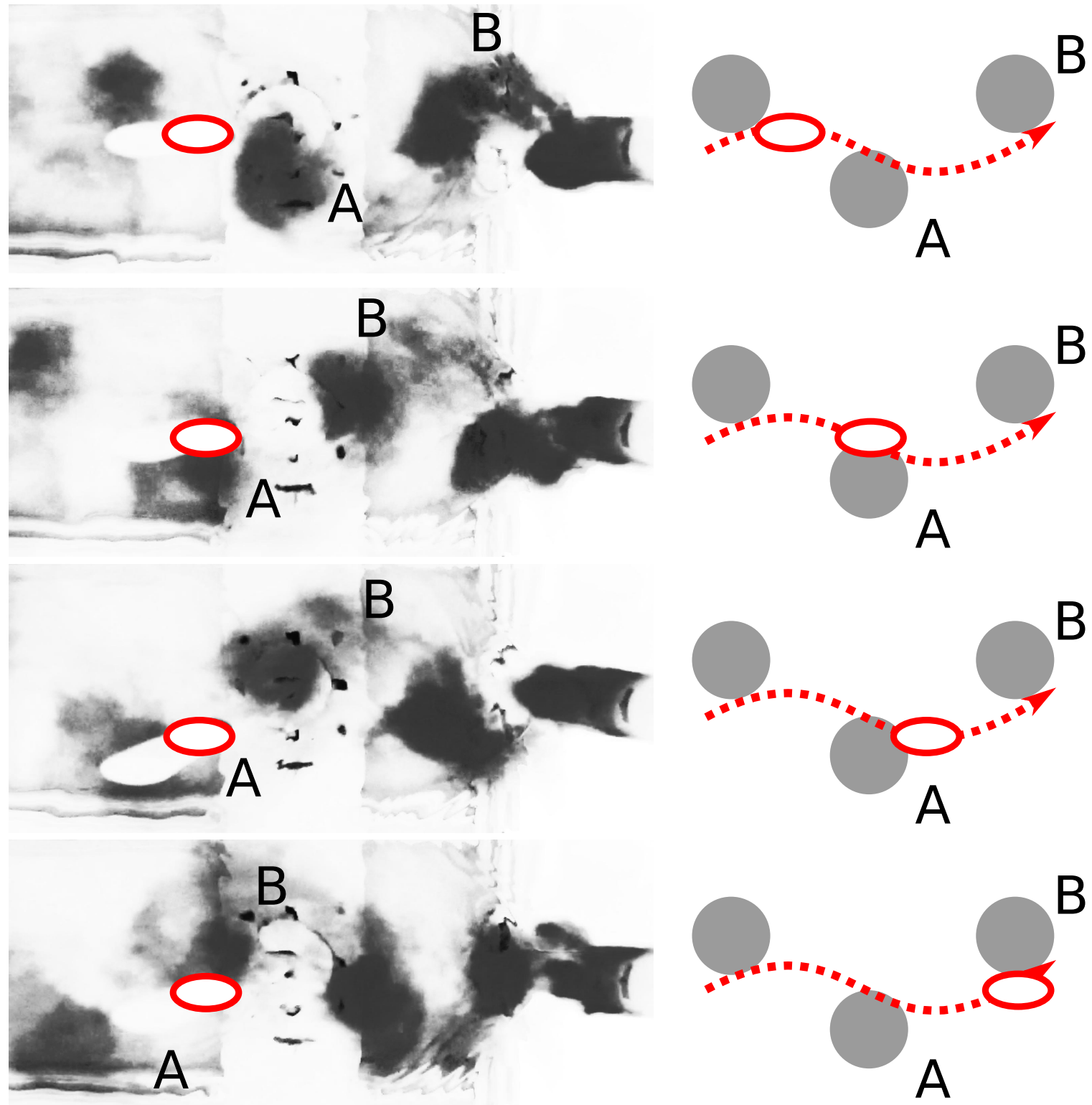

Figure 4-9: Sequence of dye visualizations (left) and schematics (right) showing the whisker's free vibration path through the vortex wake. The frequency of the whisker's motion aligns with the vortex wake frequency, as the whisker slaloms between the vortices. $\left(U^{*}=16, R e=3,180\right)$ 


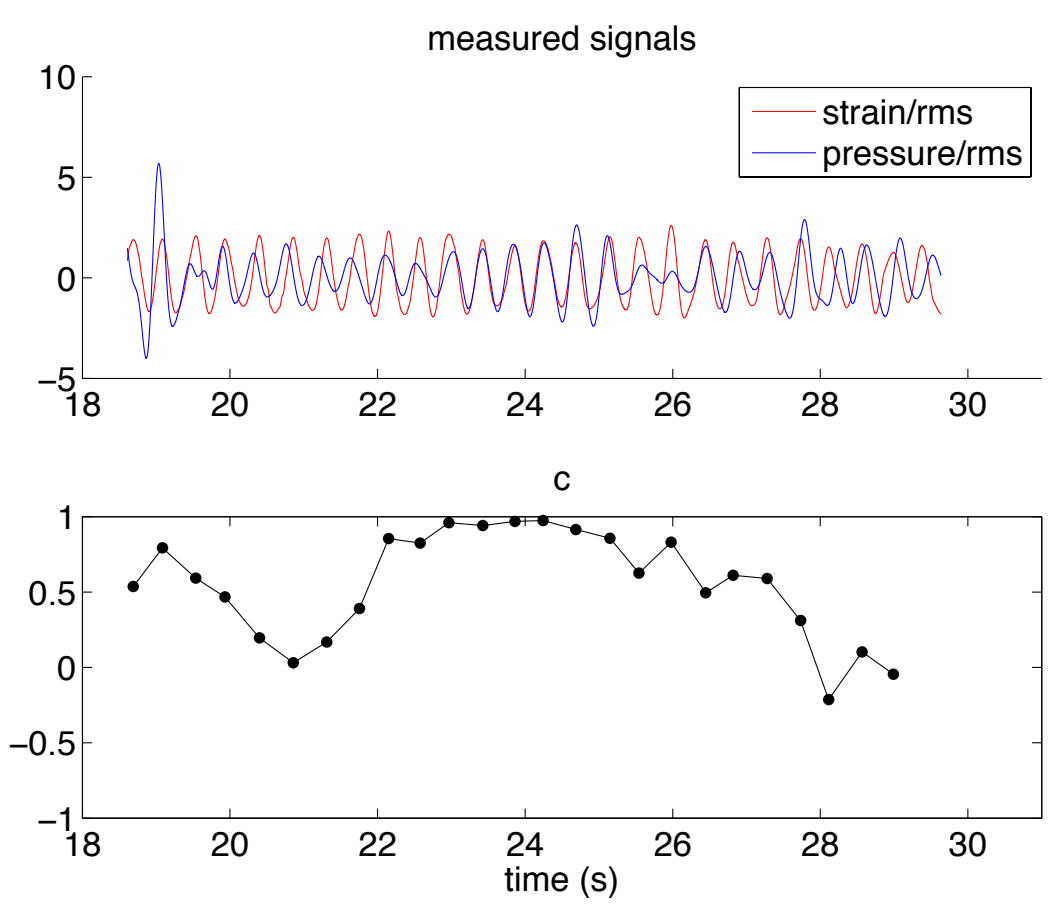

Figure 4-10: Sample result of the cross-correlation analysis. (Top) The normalized signals of strain and pressure measured on the whisker and in the wake, respectively. (Bottom) The cross-correlation coefficient at each oscillation cycle. $\left(d_{x}=17 d_{w}, d_{c y l}=\right.$ $\left.4 d_{w}, U^{*}=27\right)$

time via an initial cross-correlation. On bottom is $c$, the cross-correlation coefficient for those two signals at each oscillation cycle. In this case, there are segments of poor correlation near the beginning and end but a middle segment of the run reaches strong correlation.

This type of analysis is repeated for the following experiments. The results are presented with a few different metrics that aim to highlight the whisker's ability to detect the wake under a variety of conditions.

\section{Effect of cylinder size}

To test the effect of the size of the wake generator on the detection ability of the whisker, three different size cylinders were used $\left(d_{c y l}=\{2.5,4,11\} d_{w}\right)$. The whisker was placed at a downstream distance based on cylinder diameters $d_{x}=15 d_{c y l}$. This is to ensure that the wake would have evolved equivalently in each case.

In Figure 4-11(a), the maximum cross-correlation coefficient per run is plotted. 

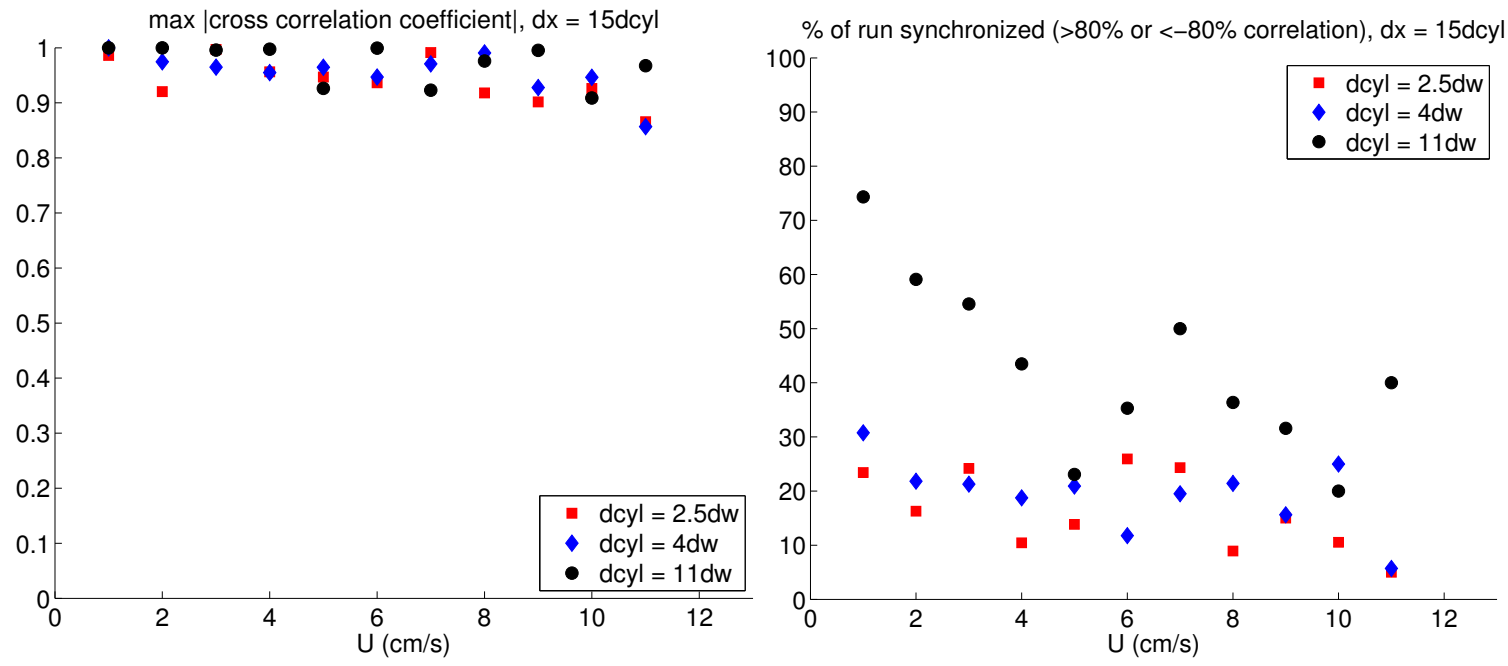

Figure 4-11: Cross-correlation metrics of the whisker vibration with the wake frequency for three different size cylinders at $d_{x}=15 d_{c y l}$. (Left) All cases see a high maximum cross-correlation coefficient. (Right) The percentage of the run where the whisker is "synchronized" generally increases with cylinder diameter.

For all sizes of cylinders tested, there is a high $(>0.85)$ maximum value. We also define a sufficient "synchronization" as when the instantaneous cross-correlation coefficient is either $>80 \%$ or $<-80 \%$. The amount of time that the whisker spends being "synchronized" with the wake is seen to vary with the size of the cylinder, and therefore the size of the dominant wake features (Figure 4-11(b)).

In Figure 4-12 the percent of the run that is "synchronized" (as averaged over all towing speeds tested) is seen to decrease with separation distance and lateral distance away from the centerline. This synchronization, however, remains present in all cases shown here, indicating that the seal could detect the presence of the wake from a distance downstream and also near the edge of the wake.

\subsubsection{Comparison between the whisker and the cylinder and elliptical geometries}

Experiments were carried out using the whisker, elliptical and circular cylinders at three downstream distances the first closer to the upstream cylinder $\left(d_{c y l}=\right.$ $\left.2.5 d_{w}, d_{x}=8 d_{w}\right)$, then moderately far away $\left(d_{x}=17 d_{w}\right)$ and finally in the far wake 


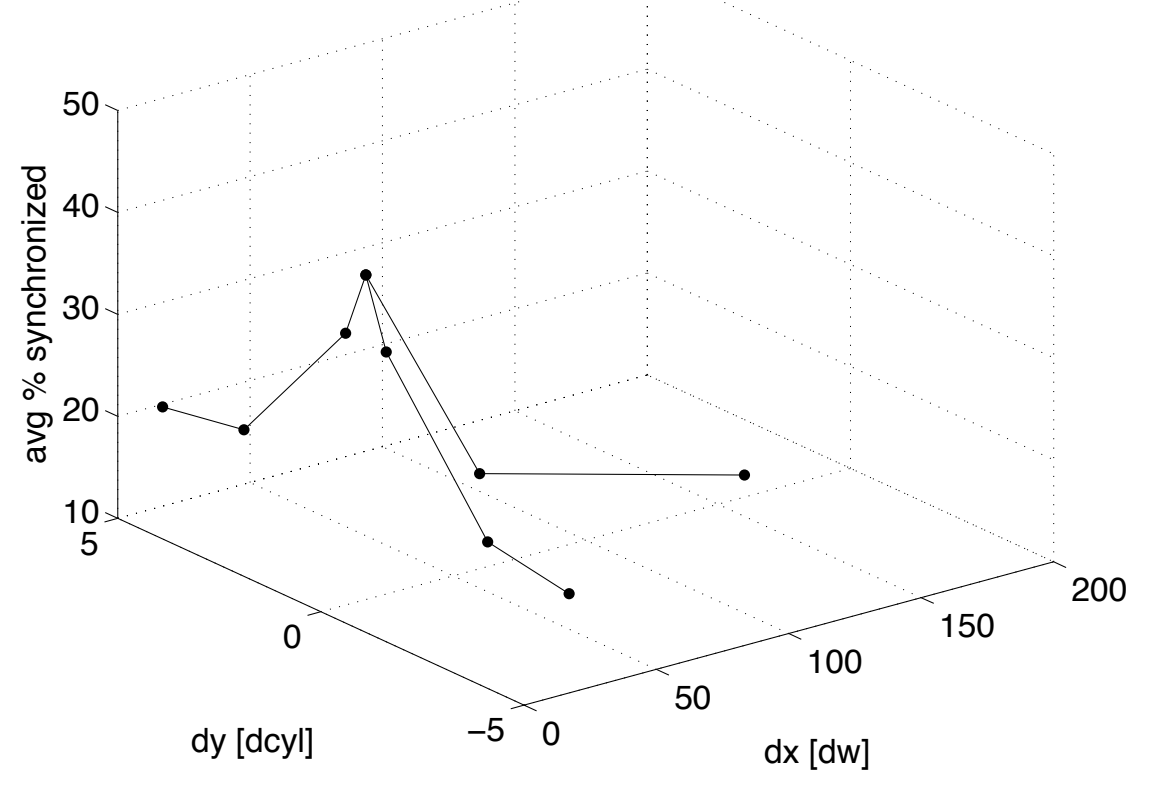

Figure 4-12: Average of percent synchronization across all speeds tested $\left(d_{c y l}=4 d_{w}\right)$. The trends are displayed versus separation distance both in the downstream $\left(d_{x}\right)$ and lateral $\left(d_{y}\right)$ directions. Experiments with a lateral offset were conducted only on one side of the cylinder but the results are assumed to be symmetric and are therefore mirrored here.

$\left(d_{x}=110 d_{w}\right)$.

The frequencies of response are shown in Figure 4-13, with $d_{x}$ increasing from left to right. At the closest distance, all geometries synchronize with the theoretical wake frequency, based on $S t=0.2$. As the distance increases to $d_{x}=17 d_{w}$, the circular cylinder deviates from the wake frequency, but the elliptical cylinder and whisker remain synchronized. When the models encounter the far wake at $d_{x}=110 d_{w}$, they exhibit different trends. The whisker locks to the upstream cylinder's Strouhal frequency over a narrow frequency range. It should be noted that the far wake develops new vortical patterns that are different than the originally shed patterns close to the cylinder [141].

The amplitudes of vibration for the three models are shown in Figure 4-14, with the circular cylinder on the left, elliptical cylinder in the middle, and whisker on the right. Results from the three different separation distances are shown, as well as the response in open water. Except at the closest distance, the circular cylinder 


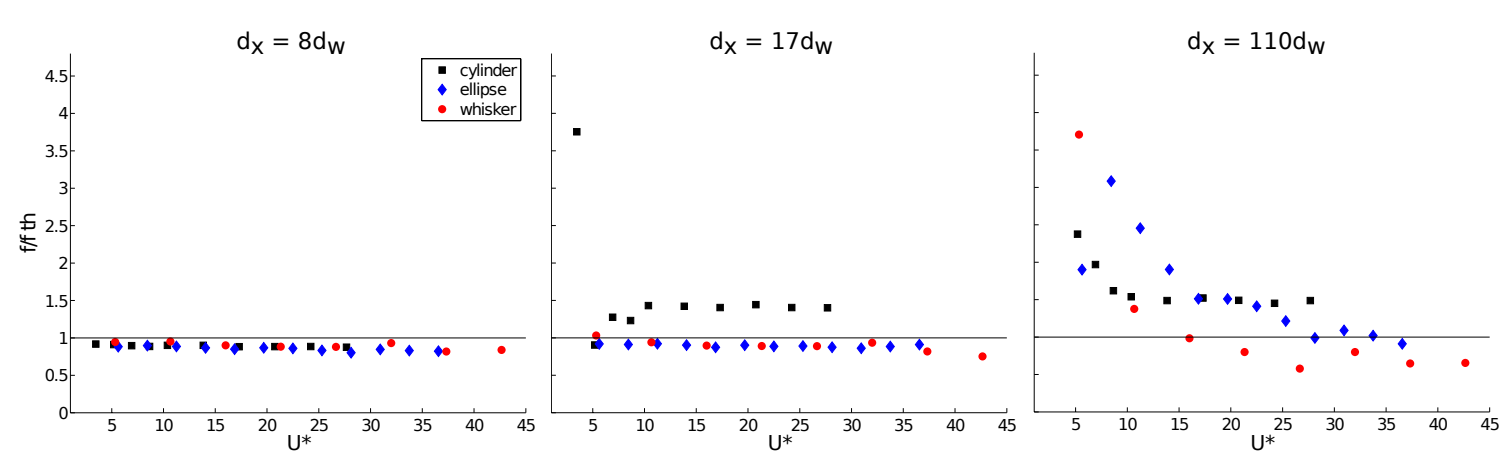

Figure 4-13: The frequencies of response nondimensionalized by the theoretical frequency. At the close distance $\left(8 d_{w}\right)$, all four models vibrate at the theoretical wake frequency. As the distance increases to $17 d_{w}$, the elliptical cylinder and whisker continue to oscillate at the wake frequency, but the cylinder deviates from it.

vibrates at roughly similar amplitudes in the wake as it does in open water. The elliptical cylinder and whisker, on the other hand, oscillate substantially more as they encounter the wake. These amplitudes generally decrease as the downstream distance increases.

At the two closest distances, all three shapes the cylinder, the elliptical cylinder, and the whisker show signs of wake induced response, which is similar to trends reported in the literature for cylinders in tandem at similar distances $d_{x} / d=2$ to 5.6 65, 5, 4, 3].

Unlike the other two models, the whisker continues to vibrate with amplitude significantly higher than its VIV response at the far distances $\{110,160\} d_{w}$. The wakeinduced vibration (WIV) amplitude of the whisker at the largest distance reaches $>7$ times higher than the VIV in open water, indicating the whisker's superior ability to detect a wake from a far distance. Testing whether the effect sustains for the longer detection distances $\left(O\left(10^{4}\right)\right.$ whisker diameters) stated in [25, 118, 138] is not possible in this facility.

\subsubsection{Wake Detectability}

The WIV frequencies and amplitudes indicate that the elliptical cylinder performs about the same or even higher than the whisker. But a distinction between the two geometries is highlighted through a detectability index, which we define as the ratio 


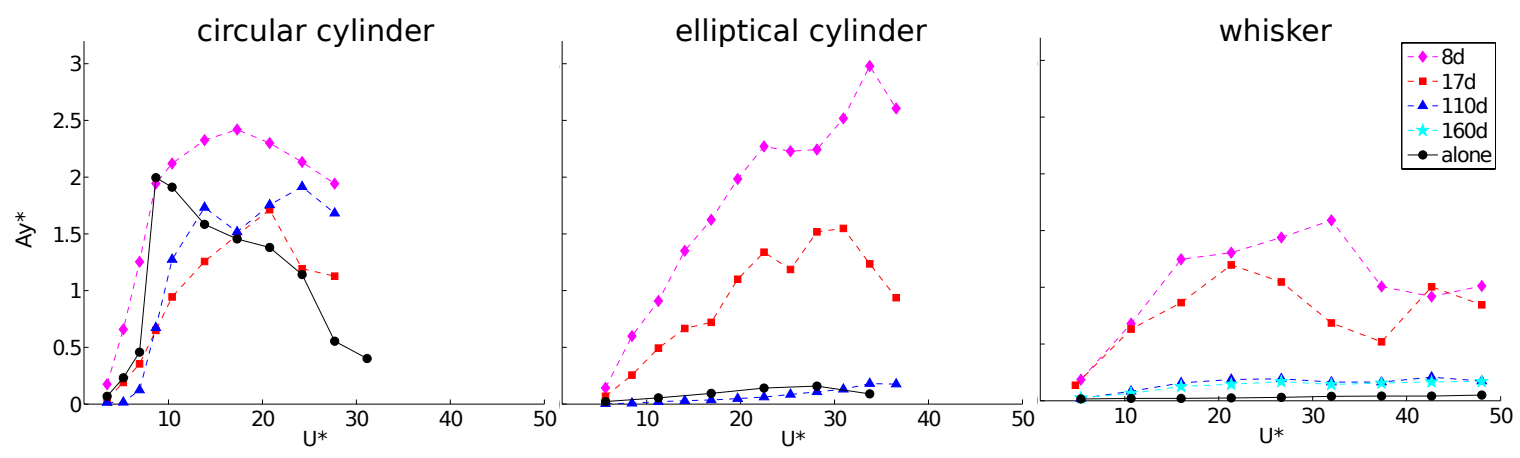

Figure 4-14: Vibration amplitudes of the circular cylinder, elliptical cylinder, and whisker (from left to right, respectively). The latter two vibrate more when they are in the wake of an upstream cylinder (colored lines) than they do in open water. The circular cylinder vibrates with a similar amplitude for all cases. The amplitudes of response decrease as the distance from the upstream cylinder increases. The whisker retains an amplitude greater than its VIV amplitude even at the largest distance.

$\Delta=\min \left(\frac{A_{\text {wake }}}{A_{\text {alone }}}\right)$. This ratio of the models' vibration amplitude in the wake and their self-induced vibrations at the same flow velocity is chosen as a metric that highlights the object's ability to detect the presence of a wake above its VIV response. The minimum ratio over all $U^{*}$ cases is chosen to be conservative. The value of that variable is plotted in Figure 4-15. The whisker's detectability is higher than that of the elliptical and circular cylinders. This inequality remains true over all of the distances tested. This is due in part to the whisker having a smaller VIV amplitude than the other two models over most of the reduced velocity range.

\subsection{Discussion}

The study of a model problem, the interaction of a harbor seal whisker model with the wake of an upstream cylinder with large diameter, provides the basic fluid mechanisms that live seals may employ when they exhibit their outstanding ability to detect and track the wakes of prey even with a $30 \mathrm{sec}$ late start. In open water the whisker model exhibits very low vibration compared to its width when its long axis is aligned with the incoming flow. However, once it enters the wake of an upstream cylinder, it oscillates with large amplitude on the order of its own width. More important, its frequency coincides with the Strouhal frequency of the upstream cylinder, hence 


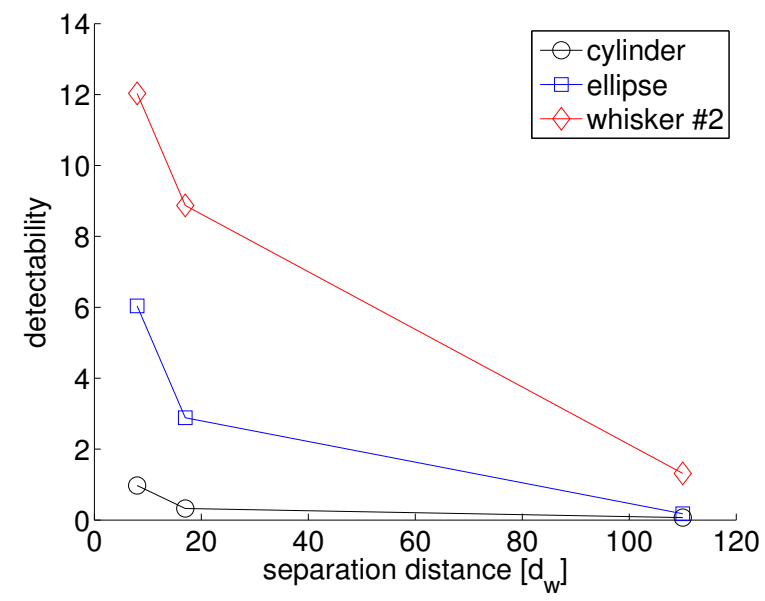

Figure 4-15: The detectability of the whisker is higher than the other models. As the separation distance increases, the wake becomes more difficult to detect, but the whisker remains the highest.

making detection of an upstream wake and the size of the wake-generating body possible.

This is a new result, which is different from the case of two interacting cylinders of comparable diameter, wherein the downstream flexibly mounted cylinder oscillates at a frequency determined by its mass and spring properties, the value of the added mass, and the wake spring constant of the wake defined in [3]. In the case of a stationary upstream cylinder with significantly larger diameter than the downstream body (2.5x, 4x, and 11x in our experiments), the flexibly mounted downstream body vibrates freely at the Strouhal frequency of the upstream cylinder, following a path that slaloms among the vortices of the oncoming wake. This path leads to significant energy extraction as also noted in the visualizations of live trout swimming behind bluff cylinders [85] and the upstream swimming of anaesthisized fish and flapping foils [7].

While a seal can swim in open flow with minimal flow noise, as shown in Chapter 2. once it enters the vortical flow of the wake, its amplitude of response increases substantially even at large distances, while its frequency locks at the frequency of the incoming vortices enabling it to detect the size of the upstream body.

In our study we employed wakes of bluff bodies to demonstrate the detection mechanisms up to a distance of 160 whisker diameters downstream of the body. 
Far wakes are known to reorganize their vortical structures creating new dominant frequencies [141], which places limits on the detectability of the specific object shape, but the presence of a distinct wake is still detectable. The use of different sized cylinders as the wake generator indicates that the whisker's detection ability tends to increase with the size of the wake features.

The study of a circular cylinder and a 2:1 elliptical cylinder serves to underline the special properties of the whisker shape. The circular cylinder responds with large amplitude even in open flow, hence making detection difficult. 


\section{Chapter 5}

\section{Conclusions}

\subsection{Overview}

Behavioral experiments with harbor seals have demonstrated their outstanding ability to detect and track hydrodynamic signatures left by swimming animals and moving objects. Despite having their auditory and visual sensory cues blocked, the seals successfully follow the paths of bodies that had swum ahead of them several seconds earlier. The seals' vibrissae (whiskers) are reported to be sensitive enough to detect even minute water movements, such as those left in the hydrodynamic trails of fish.

Various animals utilize hairlike structures as direct touch sensors. For aquatic animals that swim forward at relatively high speeds, however, it is rather surprising that they would be able to use their whiskers to sense hydrodynamic information. The cross-sectional shape of the whiskers is close in form to a bluff (non-streamlined) body, whose wake is known to spontaneously form a double array of staggered vortices, the Kármán street. The unsteady forces caused by the Kármán street result in vortexinduced vibrations (VIV) on the body, with an amplitude comparable to its crosssectional dimension and with high frequency, close to that predicted by the Strouhal law, resulting in high transverse velocity. Such vibrations would act as strong noise for the sensor, since it would be difficult to discriminate between the wake-induced fluctuations and external flow unsteadiness.

The phenomenon of VIV is ubiquitous for bluff bodies and very complex, as the 
body vibrations caused by the Kármán street alter, in turn, the vortical structures in the wake and hence the resulting forces and motions. That establishes a feedback mechanism that leads to a stable or unstable dynamic equilibrium, which varies significantly as function of the basic parameters of the problem.

In this thesis, the harbor seal whisker's ability to reduce VIV is quantified and explained. This feature is realized in a physical device. Lastly, a mechanism of vortex wake detection is elucidated. A single whisker is sufficient to detect a wake and is shown to do so passively.

\subsection{Principal Contributions}

\subsubsection{Forced vibrations on a whisker model}

Experiments with a scaled harbor seal whisker model undergoing forced vibrations demonstrate the undulatory, elliptical geometry's ability to passively reduce VIV.

\section{Low $C_{l, v}=0$ contour}

The lift coefficient in phase with velocity $\left(C_{l, v}\right)$ was measured over a range of imposed frequencies and amplitudes. The zero contour was found to be very low $(A / d<0.1)$, indicating a dramatic reduction in VIV amplitude from that of a circular cylinder at the same Reynolds number $(A / d \approx 0.7)$.

\section{Small bandwidth of response}

The added mass coefficient reveals that the whisker's bandwidth of excitation is dramatically reduced. $C_{m}$ on both circular and elliptical cylinders deviate from the potential flow solution, as the vortex shedding forces the body to lock-in over a wide

range of exception. The whisker, however, breaks down the correlation along the model span, leading to a narrower range of excitation. 


\section{Decorrelated Kárman street}

Wake visualizations of the whisker geometry help explain this dramatic reduction of VIV. The shed vortices are decorrelated, resulting in a jumbled wake, which exerts low forcing back onto the model. A Kármán street does form, but does so farther downstream than a similar model with smooth elliptical cross section.

\section{Low drag}

The whisker geometry is also seen to exhibit low drag: $C_{d} \approx 0.7$, which is even lower than that of the elliptical cylinder. Thus, the whisker is able to attain both low drag and low vibration amplitude a feat not achieved simultaneously in most VIV suppression devices.

\section{Wide range of response with angle of attack}

The VIV response of the whisker geometry changes significantly with the angle at which it faces the flow. The amplitude of response increases $\sim 20 \mathrm{x}$ as the angle of attack $(\alpha)$ increases to $90^{\circ}$. The elliptical cross section of the geometry is seen to dominate the response at higher angles, as the undulatory features are no longer large enough to have a strong effect.

This reduces the usability of this geometry as a VIV suppression device in situations where oncoming flow may approach from a range of directions. It can, however, be used to sensitively detect direction of steady oncoming flow.

\subsubsection{Design of whisker sensor}

The unique vibration properties discovered in the whisker morphology were brought into use as a sensor through two successive designs.

\section{Flow velocity and direction sensor}

Drag-based measurements are used to sense oncoming flow velocity. The wide range of response based on angle of attack allows this to also be used as a flow direction sensor. 

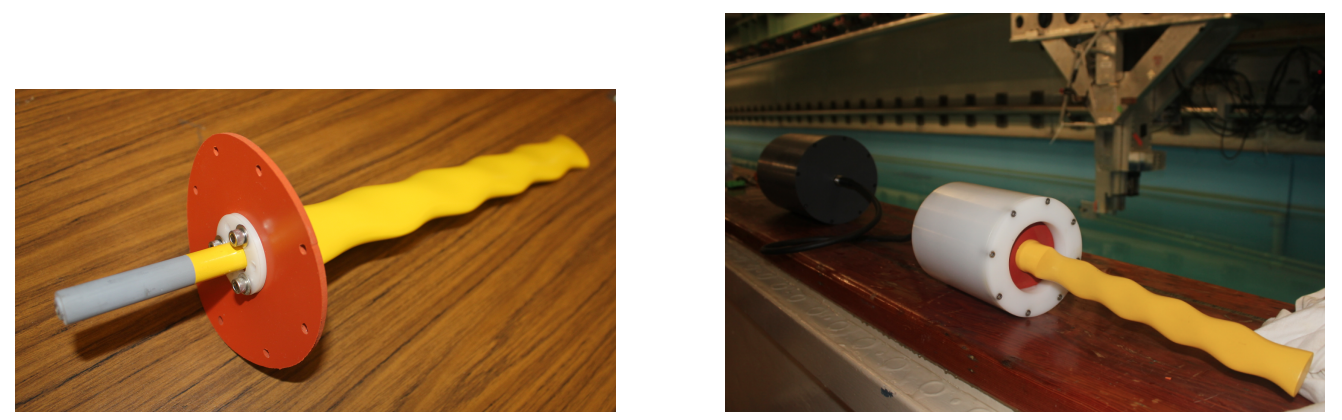

Figure 5-1: Pictures of the whisker-inspired flow velocity and direction sensor.

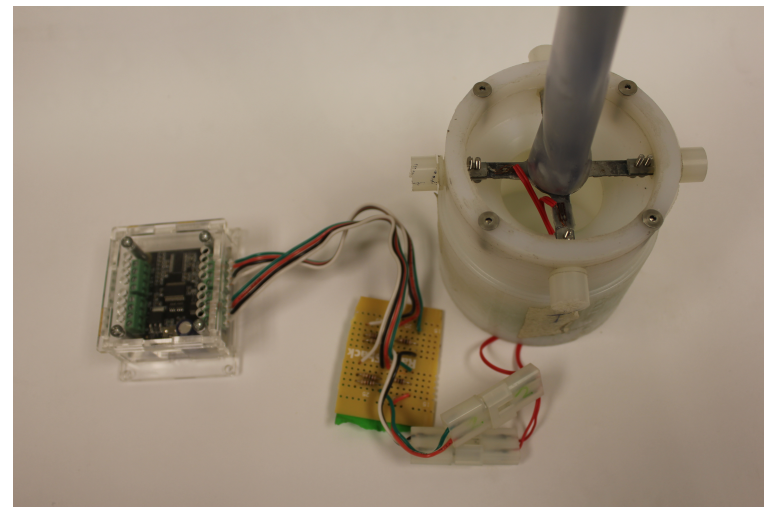

Figure 5-2: Picture of the re-designed whisker sensor, which was made for systematic testing in a vortex wake.

This is, however, not the feature that enables wake detection, which is described through the next round of experiment results.

Do seals actually use their vibrissae as velocity sensors? Perhaps they default to other sensory systems, as in [11], in which seals could maintain constant speed both with and without presence of its vibrissae. In that case, the seals may have used visual cues instead. Despite a potential preference for using other sensory systems to determine speed, the discovered whisker vibration properties still present useful sensing strategies for engineering applications.

\section{Vortex wake sensor}

An improved design is put forward for testing of vortex-wake detection. A flexurebased system allows for free vibration of the whisker model. Strain gauges are used to measure deflection at the base. 


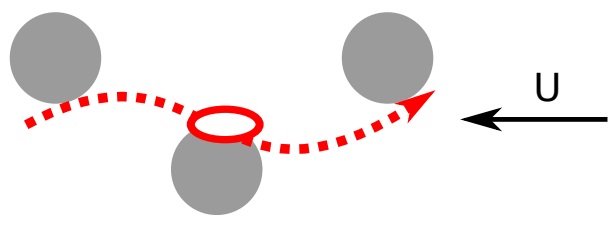

Figure 5-3: Schematic of the slaloming trajectory (dotted line) that the whisker takes as it encounters a vortex wake.

\subsubsection{Mechanism of wake detection}

Experiments with the whisker sensor undergoing free vibrations are used to describe the vibrational response of the whisker as it encounters a vortex wake. The whisker exhibits a dramatic difference in vibrational response to steady, open flow versus a vortex wake.

\section{High vibration amplitude in wake}

The whisker vibrates with much higher amplitude in a vortex wake than it does in steady flow. Depending on the distance downstream and the size ratio difference between the upstream body and the whisker, this difference can span more than an order of magnitude.

\section{Synchronization with wake frequency}

Also important is the frequency of the whisker's response. Upon entering the vortex wake, the whisker's frequency of vibration synchronizes with the vortex-shedding frequency of the wake generator. This is consistent across several distances and size ratios tested.

\section{Energy extraction}

The underlying mechanism of the whisker's response in the vortex wake resembles that seen in trout slaloming behind rocks. In both cases, the downstream body is able to move in a trajectory that allows for extract energy from the oncoming wake. 


\subsection{General Implications}

\subsubsection{Sensory systems employed}

It is unknown what information real seals rely on in actual prey pursuit conditions. Several studies provide evidence for sensory information coming from outside of the vibrissal system. For example, bioluminescence produced either by the prey or the surrounding plankton could conceivably aid a visual search [63]. Also, seals could use backlit light from the surface to aid visual sensing [63]. In one experiment [108], the seals' ability to catch live trout did not appear to be significantly affected by the removal of vibrissae. The experimental setup, however, was such that vision may have been sufficient. [96] suggests that echolocation may be more useful. Off the coast of California, seals were instrumented with accelerometers on their jaws. They measured the number of jaw motion events per day to be much larger than the number of pings that toothed whales send out. So they suggest that the acoustic method may be more effective than vibrissae. [33] proposes that the vibrissae on Arctic seals do serve a functional sensory role, as the animals had a difficult time finding ice holes when their vibrissae were restricted. Or perhaps the vibrissae can be used for orienting the seals' mouths with the prey in the last few inches, since the snout may obstruct the seals' view of the fish at that point [108].

Certainly the seals' choice of sensory system is situation-dependent. And the animals likely utilize a combination of sensory channels to successfully complete the complex behaviors required to capture prey. The mechanisms elucidated in this thesis help explain how seals can conduct wake detection from long range. More remains to be explored in determining additional ways that vibrissae can be used, either as a supplement or substitute for other types of sensory information.

\subsubsection{Comparison with other species}

As noted in [56], ecological factors may be correlated with the varying evolution of vibrissal morphology across different species. For example, the monk seal is the only 
phocid species that exclusively inhabits tropical and subtropical waters, meaning that it should be able to more heavily rely on vision in those clearer waters. Therefore its vibrissal profile perhaps did not need to evolve from smooth to beaded. Bearded seals, which also have a smooth profile, are benthic foragers and therefore likely need their whiskers more in a tactile role. Gray seals do have a beaded profile, but it is less pronounced. They are largely benthic or demersal feeders, so they also may not need to rely on their vibrissae for wake tracking.

The use of vibrissae as hydrodynamic receptors may not be uncommon at all for marine mammals. Only toothed whales (dolphins, sperm whales, etc.) have sonar systems. All others have well-developed vibrissae. Even those with whiskers that lack undulatory features, but are flattened in profile, may be able to employ the same mechanism of detection, since the data in this thesis shows the same major trends in response of the harbor seal whisker geometry compared to a smooth elliptical cylinder.

\subsection{Future Work}

Certainly this is merely the beginning of exploring the space where flow-induced vibrations and hydrodynamic perception meet. The following are a few areas of immediate interest for future studies.

\subsubsection{Optimizing the whisker geometry}

The two whisker geometries tested in this thesis were either constructed directly from provided measurements [58] or made with a single modification of a single parameter. A thorough optimization of the whisker geometry could lead to further improved VIV reduction properties. Appendix A provides images of real whiskers, which are intended to aid such a study for any interested parties.

It is interesting to note that the two geometries tested in this thesis have undulation wavelengths which align fairly well with the minima in vibration response seen in wavy cylinders, as described in Section 1.2.3. Perhaps some harbor seal vibrissae have evolved to a nondimensional wavelength of $\lambda / D_{m}=2$ and more have evolved 
to the better value of $\lambda / D_{m}=6$ - the two local minima in responses reported in [80]. Also, engineering solutions appear to have converged with biology, as the optimal wavelength to diameter ratio for strakes is about 5. Optimization of the whisker geometry would help determine what wavelength provides the global minimum in vibration response.

An attempt to design a "whiskerlike" geometry with low VIV for all angles of oncoming flow is described in Appendix B. Further attempts can be conducted to achieve a reduction greater than the $\sim 20 \%$ found there. The elliptical cross section is seen, however, to be the dominant feature responsible for VIV reduction, so it is not believed that further optimization would achieve significantly better results.

One key parameter that differs between the morphology of real whiskers and the models tested in this thesis is that of curvature. Real whiskers are curved along the span, and it would be good to determine how much the VIV response of the harbor seal whisker changes with the addition of curvature. Footage of swimming harbor seals by [95] led those authors to observe that the major curvature of the hair shaft was generally in the downstream (caudal) direction. In that case, the curvature would likely not dramatically increase the VIV response.

\subsubsection{Determining the limits of detection}

\section{Distance and time limits}

Chapter 4 showed the whisker's ability to detect the presence of a vortex wake at least 160 whisker diameters away. This was done with a relatively small wake-generating body, so it is expected that a larger object (which would more closely match the size ratio between a real whisker and a fish), would leave a stronger wake and therefore enable detection from an even further distance. The time delays reported in the behavioral experiments are $\sim 30 \mathrm{sec}$ long, which is equivalent to tens of meters. It is of interest to quantify the limit of detection, in terms of distance/time.

An order-of-magnitude argument can be made for this limit, utilizing the known sensitivity of the whiskers and the known evolution of velocities in the wake of bluff 
bodies. Based on a dipole experiment, harbor seals can detect flow speeds as low as $0.25 \mathrm{~mm} / \mathrm{s}$ [24]. The relationship of the wake-averaged defect velocity $\left(U_{0} / U\right)$ behind a bluff body versus downstream distance $(x / d)$ is shown in [123]. Given a fish swimming at $U=O\left(10^{-1}\right) \mathrm{m} / \mathrm{s}$, the seal would theoretically be able to detect $U_{0} / U=O\left(10^{-1}\right) / O\left(10^{-4}\right)=0\left(10^{-3}\right) \mathrm{m} / \mathrm{s}$. Based on the data compiled in [123], this value equates to $O\left(10^{3}\right.$ ) (using a conservative estimate of the trend for non-stratified wakes). This means that a small fish of size $O(10) \mathrm{cm}$ could be detected from $O(100)$ meters away.

\section{Other types of wakes}

In this thesis, a von Kármán street was employed as a representative wake. An efficiently swimming fish, however, will leave a reverse von Kármán street, which has vortices rotating in the opposite direction and produces thrust. It should be determined whether or not the whisker response is the same in that wake. Also, to more closely represent the real scenario, the upstream disturbance should be oriented perpendicular to the whisker length. I postulate that these two changes will not induce dramatic changes in the response, as the dominant wake features will be the same.

Additional variations in the wake, such as bifurcations, could be tested for detectability by the whisker. Fish leave a range of hydrodynamic trails depending on their kinematics, and therefore do not always leave a perfect Kármán street. Such tests may help uncover the limits of detection.

\subsubsection{Implementing a control loop}

The device developed here is able to passively detect the presence of a vortex wake. For robotics applications, the next technological hurdle is to conduct vortex-wake tracking, which requires actuation and control, but would enable localization of a moving source. The first step could be to design a basic control system to detect the location of a wake coming from an unknown but stationary position. Then the 
control could be enhanced to detect the heading of the wake and follow it in real time as the wake-generating body turns.

\subsubsection{Determining the function of the whisker array}

In this thesis, I show that a single whisker is sufficient to perform wake detection. To carry out more complex tasks, however, multiple whiskers may be required or beneficial. Studies of the interaction between the whiskers may uncover additional insight into how multiple sensory inputs can be used to conduct such tasks.

The shear number of whiskers ( $\sim 40$ on each side of the muzzle) suggests that there exists some beneficial function to having an array. It is known that multiple whiskers can be used in concert to aid discrimination of size and shape in tactile sensing, but the hydrodynamic function of the vibrissal array is a subject to be explored.

The arrangement of mystacial vibrissae in the array across the muzzle is known: There are several horizontal rows parallel to the mouth line, the vibrissae are generally longer and thicker in the posterior and ventral directions, and the lower rows tend to have more vibrissae. Muscles in the muzzle allow the seal to position the whiskers in a variety of arrangements. The position of individual whiskers during real prey pursuit is unknown. If the videos provided in [95], which show seals swimming in lab conditions, depict the same whisker positions during wake tracking, the following are a few notable features and corresponding hypotheses about the hydrodynamic implications of this arrangement. Screenshots from the videos are provided in Figure 5-4.

- The "fan" arrangement of the array places the whiskers at many different angles to the horizontal plane. This is likely done to cover a wide area of the flow. The row or individual whisker that vibrates the most can indicate which direction the wake is coming from.

- It appears that despite being protracted into a "fan", all whiskers remain facing the flow in their streamlined orientation $\left(\alpha \approx 0^{\circ}\right)$, indicating that they will all experience low VIV. 


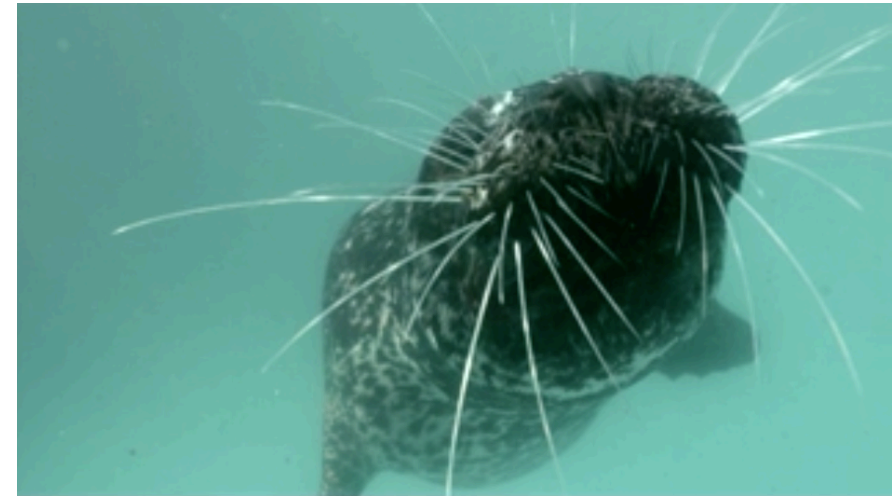

(a)

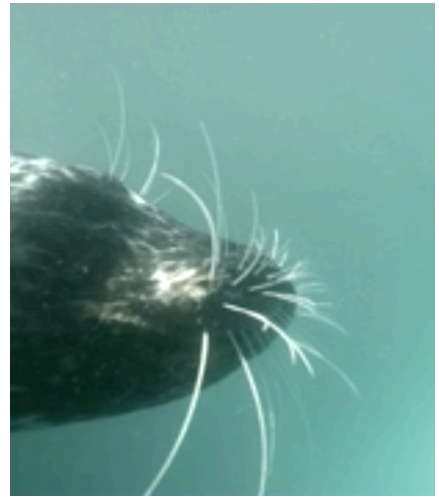

(b)

Figure 5-4: (a) Front and (b) side views of the arrangement of whiskers as a harbor seal swims forward. Screenshots taken from supplementary video in [95].

- Along each row, the whiskers increase in length. This could be used to estimate the size of a flow feature, such as a vortex which may hit a short whisker but not the tip of a long one.

- Alternatively, the range in lengths of the whiskers could exist to help determine frequency information. As whiskers with different lengths will have different natural frequencies, each has a different range that it will respond best to.

- The curvature of the whiskers within one row generally increases from anterior to posterior. This could possibly serve to reduce wake effects generated by the upstream whiskers felt on the downstream ones.

- The most anterior whiskers, which are the shortest, may not play a major role in hydrodynamic sensing, as they do not reach far out into the flow. Perhaps they are mostly used for fine tactile sensing. As seen in [19], a sea lion first used the longer vibrissae to locate an object and then the shorter ones on top of its snout to collect finer details about the shape.

More information about the whiskers' response under real prey pursuit conditions remains to be uncovered. A fine-resolution view of the whiskers encountering various hydrodynamic trails will greatly aid our understanding of how the array may be employed for sensing. 


\section{Appendix A}

\section{Whisker Image Database}

This appendix compiles several pictures of real harbor seal whiskers. These images are intended to serve as a tool for further studies of the whisker shape, which may include the goal of optimization for VIV suppression.

Figures A-1 and A-2 are pictures of five different whiskers shed by harbor seals at the New England Aquarium. The whiskers were mounted vertically in a water tank. Images were taken using digital holography (DH) with the help of Yi Liu in the MIT Optics Lab. Back-propagation was used to focus the images at the correct plane. Each whisker was captured in multiple sections due to the long whisker length relative to the camera field of view.

Figure A-3 is an image from [95], which shows cross sections of different seal species' whiskers based on CT scans. All species show flattening towards the tip. The sea lion whisker is essentially an elliptical cylinder the whole way down the span and the latter two show the undulatory features along the span by alternating from a roughly circular to elliptical cross section.

Images and dimensions are also provided in [58], but they are not replicated here since there may be an error in the wavelength reported. Finally, some dimensions are given in [46, 45], which are copied over in Figures A-4 A-5, and A-6. 

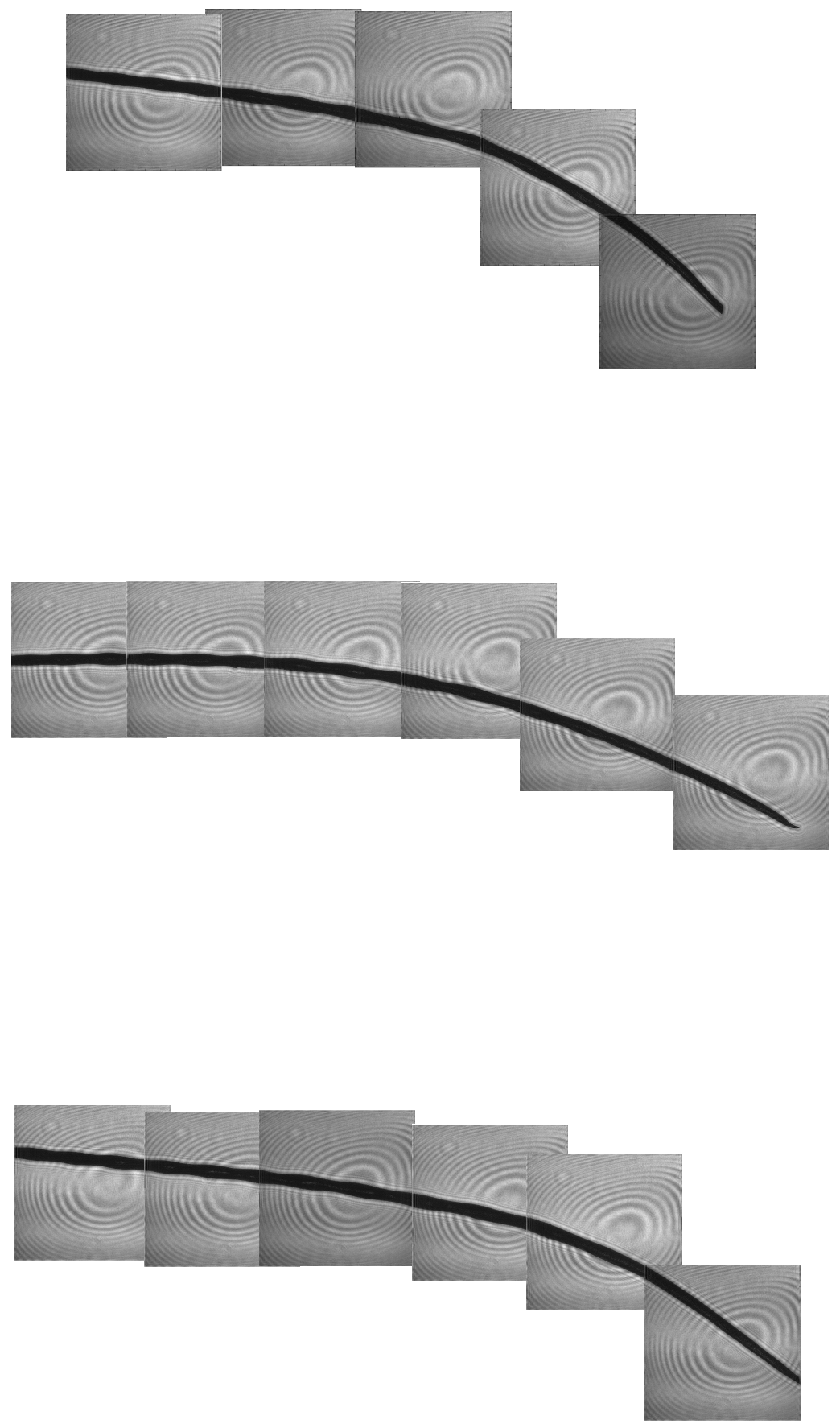

Figure A-1: Digital holographic images of whiskers from a harbor seal at the New England Aquarium. All are in the $90^{\circ}$ configuration. The camera field of view and therefore the dimensions of each DH image box are $1.23 \times 1.23 \mathrm{~cm}$. 

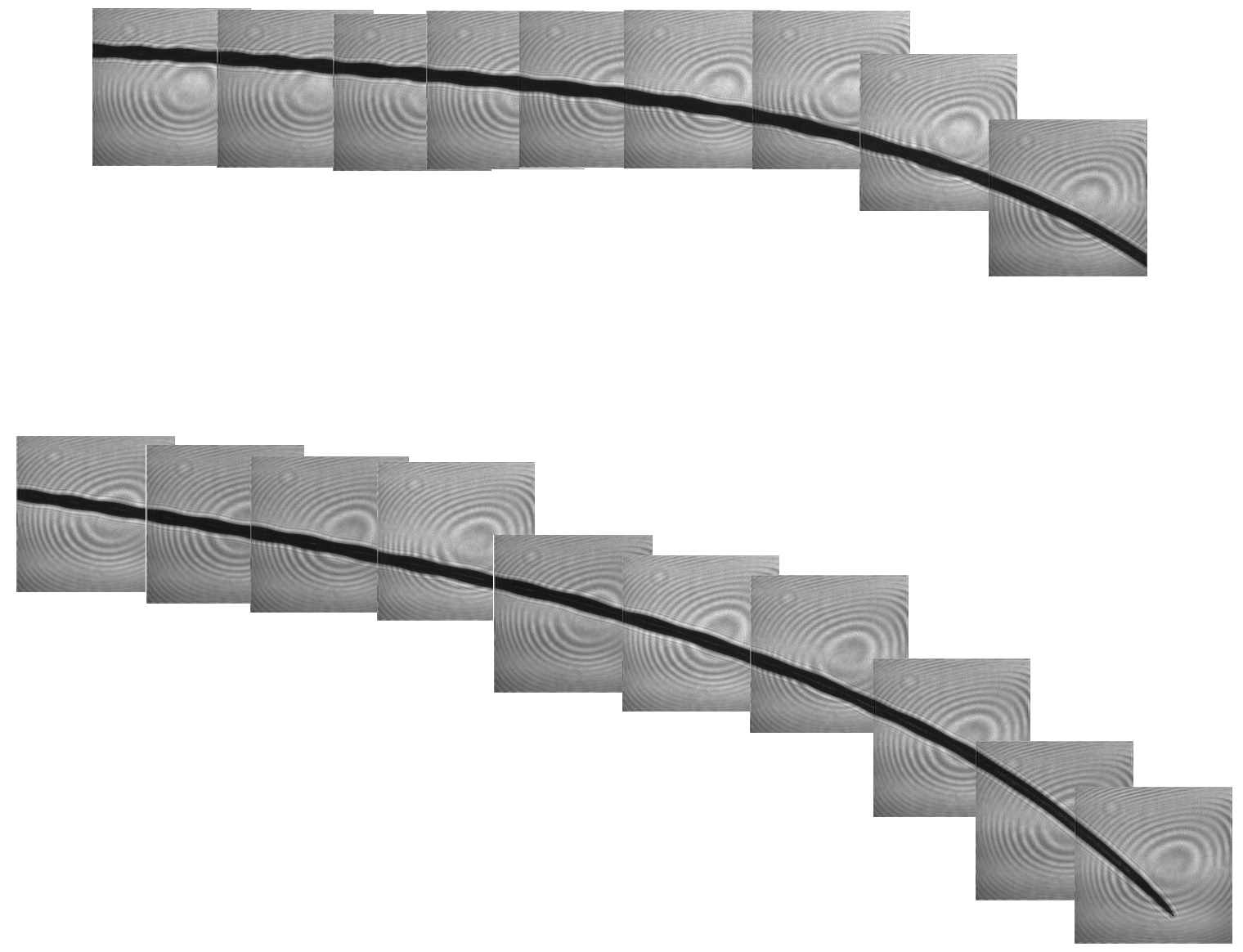

Figure A-2: Digital holographic images of whiskers from a harbor seal at the New England Aquarium. All are in the $90^{\circ}$ configuration. The camera field of view and therefore the dimensions of each DH image box are $1.23 \times 1.23 \mathrm{~cm}$. 

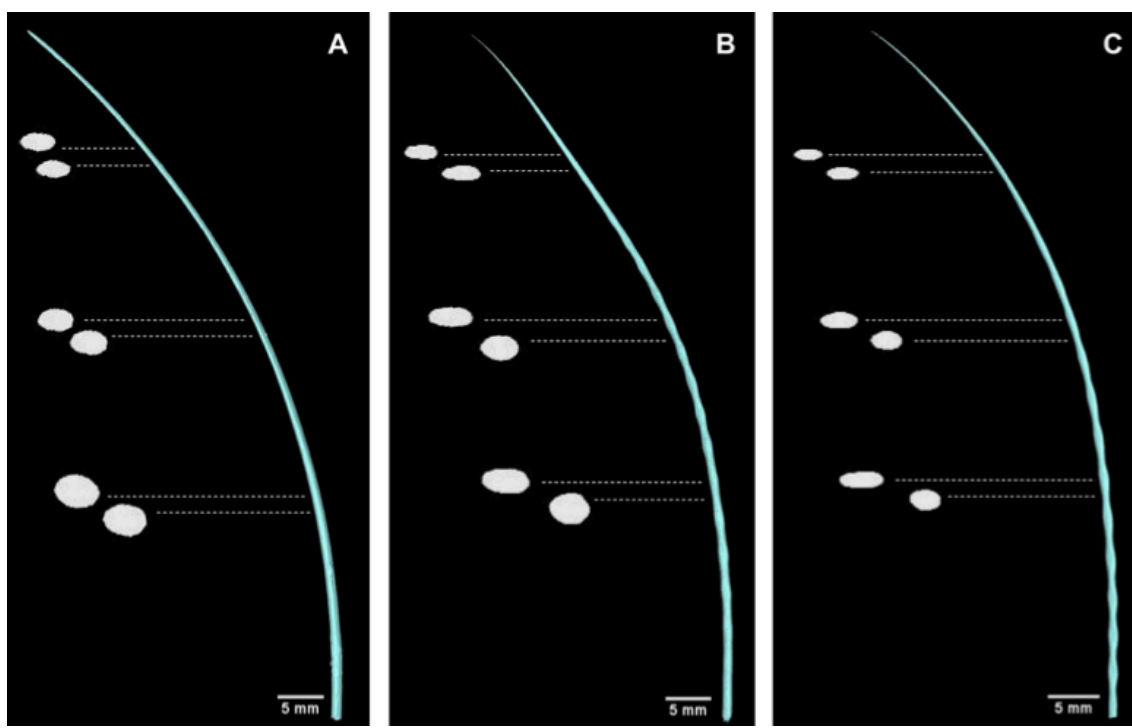

Figure A-3: From CT scans, these are reconstructions of (A) sea lion (B) elephant seal and $(\mathrm{C})$ harbor seal vibrissae. Cross sections are enlarged and shown at 6 different locations along the span. Figure from [95]

\begin{tabular}{llccc}
\hline \hline & & P. groenlandicus & C. cristata & H. grypus \\
\hline Number of Vibrissae & & $33-41(38 \pm 4)$ & $24-39(32 \pm 11)$ & 25 \\
Overall length (mm) & & $39.3 \pm 17.3$ & $33.4 \pm 16.8$ & $37.8 \pm 13.1$ \\
Total bumps & & $12 \pm 5$ & $10 \pm 6$ & $9 \pm 3$ \\
Peak-to-peak & Proximal & $2.65 \pm 0.42$ & $2.24 \pm 0.13$ & $3.50 \pm 0.40$ \\
distance (mm) & Middle & $3.00 \pm 0.42$ & $2.50 \pm 0.48$ & $3.90 \pm 0.37$ \\
& Distal & $2.70 \pm 0.55$ & $2.26 \pm 0.24$ & $3.80 \pm 0.57$ \\
& Tip & $2.50 \pm 0.50$ & $2.00 \pm 0.34$ & $3.90 \pm 0.49$ \\
Crest width (mm) & Proximal & $0.63 \pm 0.07$ & $0.58 \pm 0.08$ & $0.94 \pm 0.17$ \\
& Middle & $0.61 \pm 0.04$ & $0.57 \pm 0.09$ & $0.97 \pm 0.19$ \\
& Distal & $0.45 \pm 0.08$ & $0.45 \pm 0.06$ & $0.89 \pm 0.18$ \\
Trough width (mm) & Tip & $0.34 \pm 0.06$ & $0.31 \pm 0.06$ & $0.91 \pm 0.15$ \\
& Proximal & $0.47 \pm 0.06$ & $0.46 \pm 0.09$ & $0.80 \pm 0.16$ \\
& Middle & $0.43 \pm 0.05$ & $0.42 \pm 0.07$ & $0.76 \pm 0.15$ \\
& Distal & $0.33 \pm 0.07$ & $0.36 \pm 0.04$ & $0.72 \pm 0.14$ \\
& Tip & $0.25 \pm 0.06$ & $0.25 \pm 0.06$ & $0.74 \pm 0.14$ \\
\hline
\end{tabular}

Figure A-4: Table showing measured geometric parameters of the vibrissae of these three species: harp, hooded, and grey from left to right. Figure from [46]. 


\begin{tabular}{|c|c|c|c|c|c|c|c|}
\hline & $\begin{array}{l}\text { Top Peak-to-Peak } \\
\text { Distance }(\mathrm{mm})\end{array}$ & $\begin{array}{l}\text { Bottom Peak-to- } \\
\text { Peak Distance (mm) }\end{array}$ & Crest Width (mm) & $\begin{array}{l}\text { Trough Width } \\
\text { (mm) }\end{array}$ & $\begin{array}{l}\text { Crest Width/ } \\
\text { Trough Width }\end{array}$ & Total Length $(\mathrm{mm})$ & \# of Beads/cm \\
\hline Harp & $3.97 \pm 0.70$ & $3.94 \pm 0.69$ & $0.88 \pm 0.14$ & $0.69 \pm 0.13$ & 1.28 & $80.6 \pm 10.8^{\mathrm{ab}}$ & $2.2 \pm 0.4^{b c}$ \\
\hline Harbor & $3.27 \pm 0.39$ & $3.26 \pm 0.40$ & $0.92 \pm 0.13$ & $0.73 \pm 0.12$ & 1.26 & $86.5 \pm 10.5^{\mathrm{ab}}$ & $2.3 \pm 0.4^{\mathrm{abc}}$ \\
\hline Ringed & $3.56 \pm 0.73$ & $3.53 \pm 0.72$ & $0.70 \pm 0.21$ & $0.49 \pm 0.21$ & 1.44 & $79.6 \pm 10.9^{\mathrm{ab}}$ & $2.5 \pm 0.5^{\mathrm{ab}}$ \\
\hline Spotted & $4.01 \pm 0.63$ & $3.99 \pm 0.63$ & $1.04 \pm 0.15$ & $0.85 \pm 0.15$ & 1.22 & $91.2 \pm 7.9^{\mathrm{a}}$ & $1.9 \pm 0.2^{c}$ \\
\hline Gray & $3.43 \pm 0.38$ & $3.41 \pm 0.39$ & $0.76 \pm 0.13$ & $0.63 \pm 0.11$ & 1.21 & $73.8 \pm 12.5^{b}$ & $2.7 \pm 0.5^{a}$ \\
\hline Bearded & $3.64 \pm 0.66$ & $3.62 \pm 0.65$ & $1.18 \pm 0.27$ & $1.18 \pm 0.27$ & 0.99 & $77.2 \pm 9.5^{\mathrm{ab}}$ & 0 \\
\hline Fur Seals & $3.64 \pm 0.66$ & $3.62 \pm 0.65$ & $0.96 \pm 0.12$ & $0.96 \pm 0.12$ & 1.01 & $83.0 \pm 20.3^{\mathrm{ab}}$ & 0 \\
\hline Sea Lions & $3.64 \pm 0.66$ & $3.62 \pm 0.65$ & $0.84 \pm 0.19$ & $0.84 \pm 0.19$ & 1.00 & $90.7 \pm 11.3^{\mathrm{a}}$ & 0 \\
\hline
\end{tabular}

Mean \pm SD values for each species are given. Significant differences between species for total length and number of beads per $\mathrm{cm}$ are indicated by different letters. Significant differences between species for the other traditional morphometric measurements are given in Figure 3 . Species with different letters are significantly different from one another.

doi:10.1371/journal.pone.0034481.t002

Figure A-5: Table showing measured geometric parameters of the vibrissae of multiple species. Figure from [45].
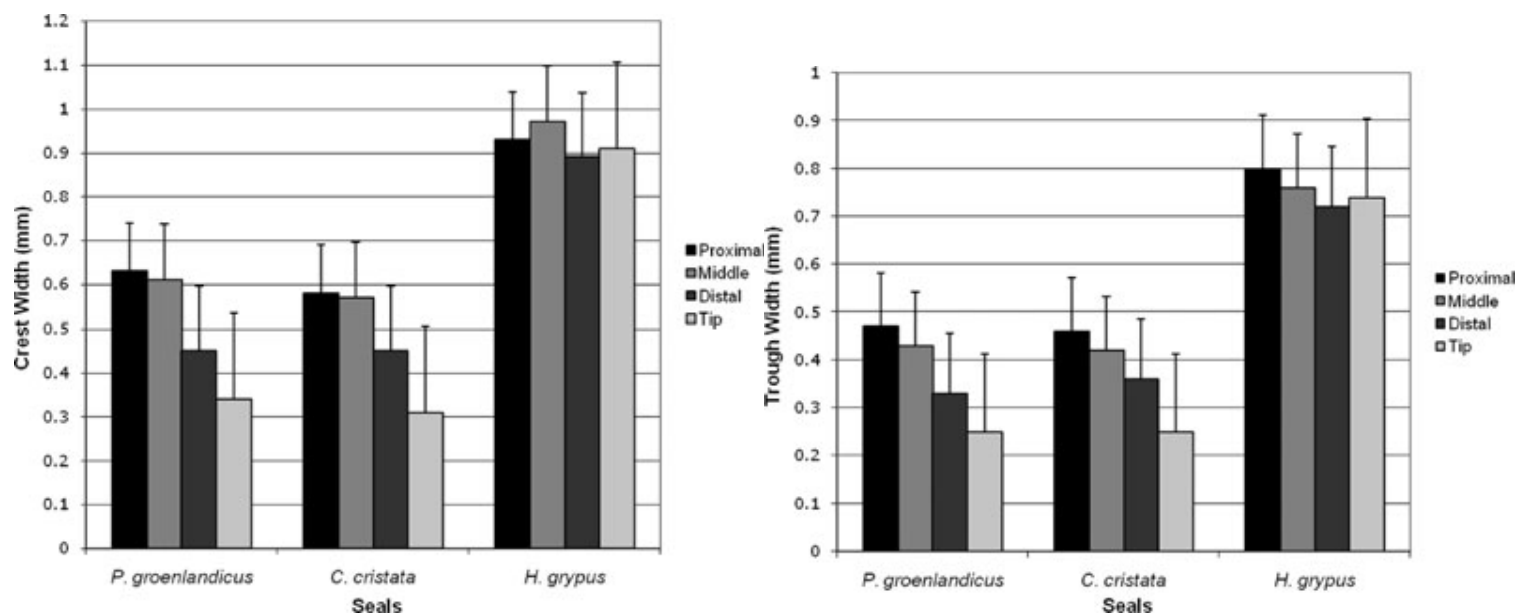

Figure A-6: (a) Crest width and (b) trough width measured on vibrissae of different species. The diameter decreases towards the tip. Figure from [46]. 


\title{
Appendix B
}

\section{Omnidirectional Whisker-Inspired Geometries}

\author{
Can a "whiskerlike" geometry be designed to reduce VIV \\ regardless of oncoming flow angle?
}

\section{B.1 Experiment Motivation}

A wide range of vibrational response over all angles of attack can be useful for a sensor, but it is a poor feature for a VIV reduction mechanism, particularly in the ocean where currents may come from multiple directions. Therefore, it is desired to know if a "whiskerlike" geometry can be created to experience low VIV over a wide range of flow directions.

\section{B.2 Shape Design}

Seven geometries were designed, based on various features of the original whisker shape. They were fabricated into plastic via stereolithography, a layer-by-layer process. Each model has the same length ratio $(L / d)$ as the original whisker and is made from the same material. Their mass ratios range from $m^{*}=0.9$ to 1.8 . 


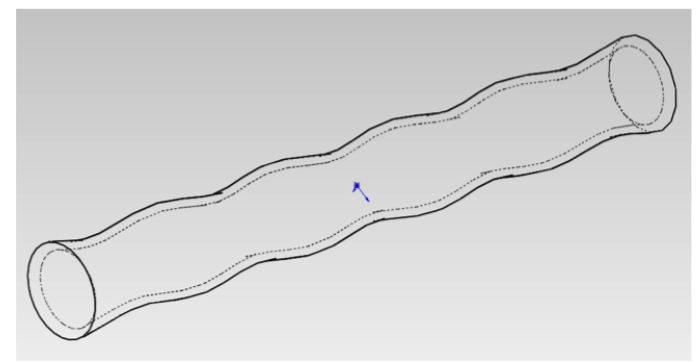

(a)

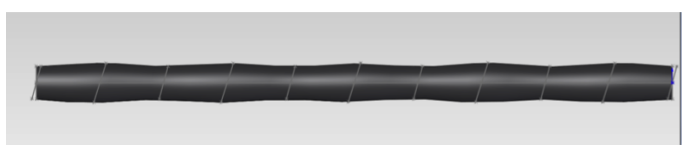

(b)

Figure B-1: Screenshots of Model 1 and Model 2, which were designed keeping all dimensions the same as the original whisker except that one dimension of each cross section was expanded or shrunk to make it circular.

\section{B.2.1 Direct Conversion to Circular cross sections}

The first two (Model 1 and Model 2) take the cross-section dimensions provided in [58] and change one of the two diameters such that each cross section becomes circular. The same wavelength as the original whisker is maintained. See Figure B-1a and B-1b,

\section{B.2.2 "Spiral-like" Shapes}

The next four (Models 3, 4, 5, and 6) are created via parametric equations. The equation for a standard spiral (Equation B.1) is repeated multiple times, each time with slight offset in the spanwise $(z)$ direction and in amplitude. Put together, these "strips" of helices form a spiral body with undulating amplitude. The base helix has amplitude $(a)$ of $0.475 \mathrm{~mm}$, the smallest radius of the whisker. As shown in Equation B.2, over each one of $n$ iterations, the amplitude is increased by a constant amount $(\Delta a)$ up to the maximum radius of $0.595 \mathrm{~mm}$, following the dimensions of the whisker given in [58]. The $z$ offset is also increased by a constant amount (half wavelength/number of iterations) in each iteration. The same process is then repeated with decreasing amplitude to create strips of helices back down to the base spiral radius but with increasing offset in the $z$ direction (Equation B.3). This strip method is depicted visually in Figure B-2. 


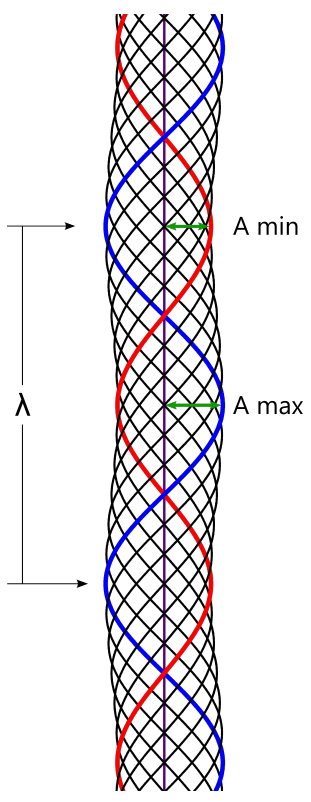

Figure B-2: Spiral strips combined together. The blue and red lines are the initial and final spirals. Wavelength $(\lambda)$ and the two amplitudes $\left(A_{\min }\right.$ and $\left.A_{\max }\right)$ are labeled.

$$
\begin{aligned}
& x=a \cos (\omega t) \\
& y=a \sin (\omega t) \\
& z=t
\end{aligned}
$$

$$
\begin{aligned}
& a=a+\Delta a \\
& z=z+\frac{\lambda}{n}
\end{aligned}
$$

$$
\begin{aligned}
& a=a-\Delta a \\
& z=z+\frac{\lambda}{n}
\end{aligned}
$$

Model 3 follows the same wavelength as that of the previous two models and the 


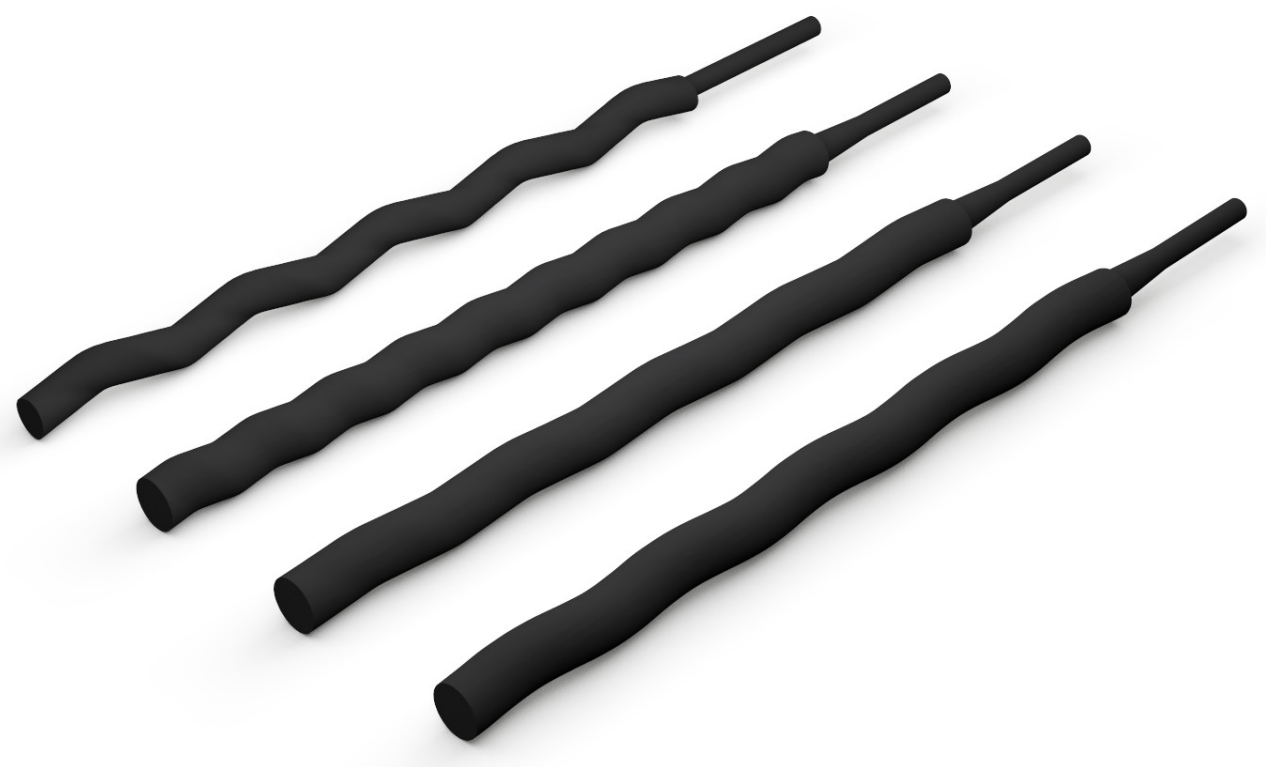

Figure B-3: All the models created via parametric equations. From the left: Model 5, Model 4, Model 3, Model 6.

original whisker dimension. Model 4 uses half of the wavelength. Model 5 was created using the same parametric equations as above, but now with a more extreme change in diameter. The overall minimum and maximum radii of the actual whisker $(0.24 \mathrm{~mm}$ and $0.595 \mathrm{~mm}$ ) were used as the base and top radii of the spiral body, in order to create a model with more pronounced undulations. Model 6 was created using the original whisker dimensions, but with a sinusoidal change in amplitude rather than a linear one.

The coordinates generated from these equations are then meshed together via MeshPlot, imported into SolidWorks, scaled up to match the mean cross-stream dimension of the previous models $(\sim 1$ in $)$, and fabricated. Figure B-3 shows all the models created via the parametric equations.

\section{B.2.3 Twisted Ellipse}

The final model generated is that of a twisted elliptical cylinder. This geometry was created by taking the average elliptical cross section of the whisker geometry and twisting it along the spanwise axis. The amount of twist applied was chosen such 


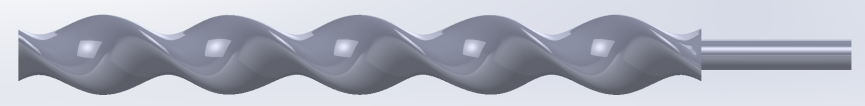

SolidWorks Educational Edition.

For Instructional Use Only.

Figure B-4: The twisted elliptical cylinder, as generated in Solidworks

that the wavelength of undulations matched that of the whisker. This value is given by $\phi=2.95 z / d$, where $z$ is the span wise position. A picture of the model is shown in Figure $\mathrm{B}-4$.

Work by [76] numerically tests the forces felt on a stationary body with a similar shape. Their geometry was also generated by twisting an elliptical cylinder, but it has slightly different parameters: $A / d_{\text {avg }}=0.1, \lambda / d_{\text {avg }}=\pi / 3=1.05, \phi=2 z / d$, $R e=3,900$, where $A$ is the amplitude of undulation. In my case, those parameters are $A_{1} / d_{\text {avg }}=0.67, A_{2} / d_{\text {avg }}=0.33, \lambda / d_{\text {avg }}=1.14, \phi=2.95 z / d$, and $R e=10,800$. These geometrical parameters do not differ dramatically, so the results from the two models can be broadly compared.

\section{B.3 Forced Vibrations: Force Measurements}

\section{B.3.1 Apparatus}

Forces were measured as the models underwent a range of imposed motions. The hydrodynamic forces are used to calculate nondimensional coefficients that describe the real vibrational response. The same linear motor and force sensor apparatus used 

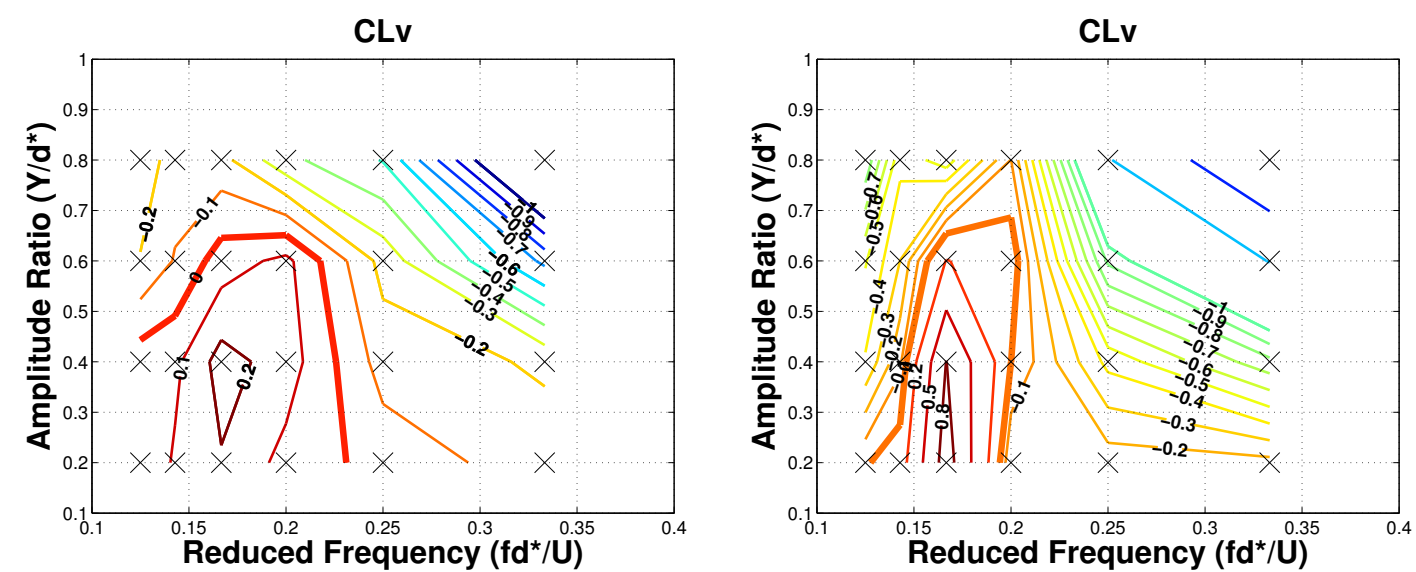

Figure B-5: $C_{l, v}$ contours of Models 1 and 2.

in Chapter 2 was employed for the present experiments.

\section{B.4 Results}

\section{B.4.1 $C_{l, v}$}

The zero $C_{l, v}$ contour is used to describe the VIV response of the geometry under zero damping conditions. The peak therefore indicates the peak expected VIV amplitude.

Models 1 and 2, which are shown in Figure B-5 have a peak amplitude of $\sim 0.65$. Both of those first two models thus present a reduction in VIV amplitude of about $20 \%$ compared to that of the smooth circular cylinder shown in Figure 2-8.

Results from the models created via parametric equations are shown in Figure B-6. Similar to above, the peaks fall between $A / d^{*} *=0.65$ to 0.8 .

Finally, the $C_{l, v}$ contour for Model 7, the twisted ellipse, is shown in Figure B-7. The peak amplitude is found to be $A / d^{*} \approx 0.6$.

The zero contour peaks for all of the models tested in this study are similar to that of a circular cylinder, based on literature [49] and my results shown in Figure 2-8. The maximum reduction seen is in the twisted ellipse, with a reduction of $\sim 15 \%$. None of them achieve the significant VIV reduction seen in the whisker or smooth elliptical cylinder. Also, these results show a weaker reduction compared to that 

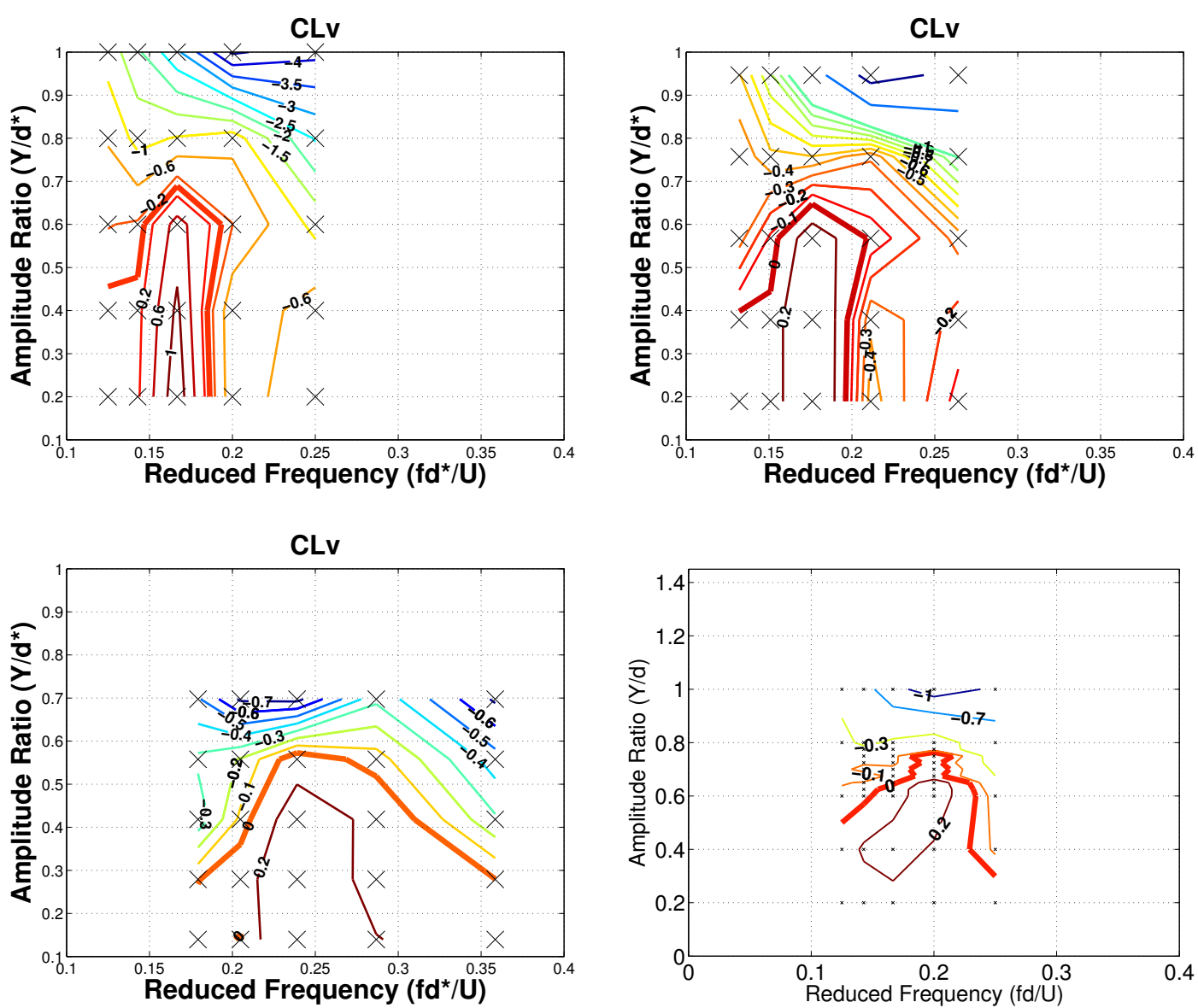

Figure B-6: $C_{l, v}$ contours of Models 3, 4, 5, 6 .

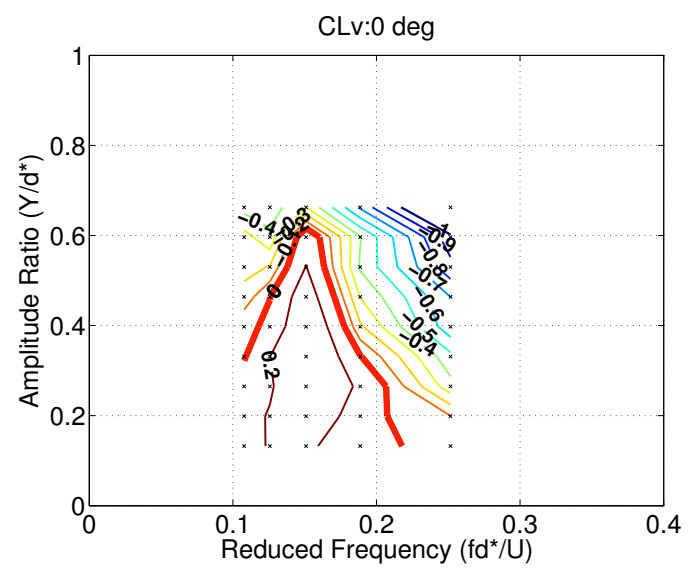

Figure B-7: $C_{l, v}$ contours of Model 7. 
in [76], which reports a reduction in mean drag up to $14 \%$ and reduction in root mean square $(r m s)$ of fluctuating lift up to $74 \%$. This large difference highlights the importance of including dynamic motion in testing, as noted in [99]. Allowing body motions to develop can strongly affect the resulting drag and lift forces experienced. 


\section{Appendix C}

\section{Effect of Flexibility on VIV}

How does flexibility affect the whiskers' vibrations?

\section{C.1 Motivation}

Real pinniped vibrissae are somewhat flexible. All of the experiments previously described in this thesis were conducted on rigid models. It is desired to know how flexibility may change the vibration properties of the harbor seal whisker geometry. Flexible circular cylinders are known to experience different VIV responses than rigid ones, including features such as vibrating along the span.

\section{C.2 Experiment Setup}

\section{C.2.1 Model Fabrication}

The following is an overview of the experiment design and fabrication. The full details can be found in Christopher Gerber's Bachelor's Thesis [44].

The experimental setup was designed around the following parameters: compliant material, adjustable natural frequency, and minimal deformation as a result of that adjustment. The chosen solution was to cast a long, flexible rubber model with two separate Kevlar strings running down the center. The high strength of the Kevlar 


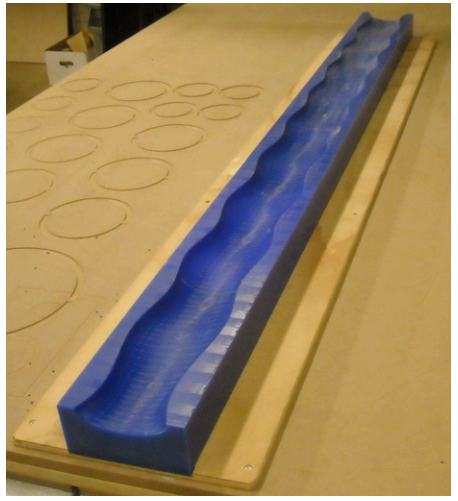

(a)

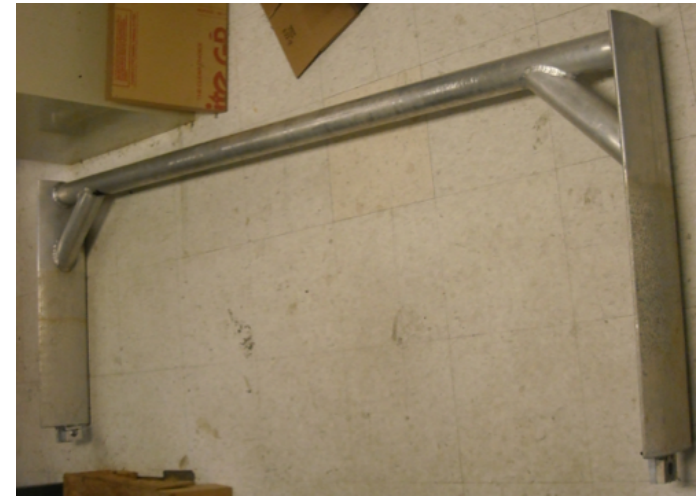

(b)

Figure C-1: (a) One half of the whisker mold, which was made from six blocks of machinable wax bolted together. A CNC tool path for the whisker geometry was created in MasterCAM from Solidworks and then routed out. (b) The mount to which the whisker was attached. The model was strung in the empty space pictured at the bottom. The two struts have an airfoil profile to minimize drag. The tube on top attaches directly to the front of the Tow Tank carriage.

allowed the model to be held taut in a base position, and turnbuckles were used to tension it to a desired level. The flexibility of the rubber allows vibrations to develop along the body.

The whisker geometry dimensions given in [58] were used directly, and scaled up $100 \mathrm{x}$, giving an average crossflow diameter of $d=5.3 \mathrm{~cm}$. A mold with the negative of this geometry was made out of machinable wax using a CNC mill (Figure C-1a). A two-part rubber (Smooth-On PMC 724) was cast into the mold to create the whisker model.

The length of the model $(6 f t)$ was chosen such that it would fit an existing mount (Figure C-1b) that was used for previous studies in the MIT Towing Tank. The diameter was chosen such that the aspect ratio would be at least 20 to allow for potential motion along the span. Also, it was chosen such that embedded sensors ( $0.5 \mathrm{in}$ cubes) would be small compared to the model, to add minimal rigidity to the otherwise flexible structure.

Accelerometers (Kistler 8688A5, +/ - 5g) were placed inside the model and used to measure model vibration without disturbing the flow, as a string potentiometer would, for example. Three of them were embedded in the center of the model during 


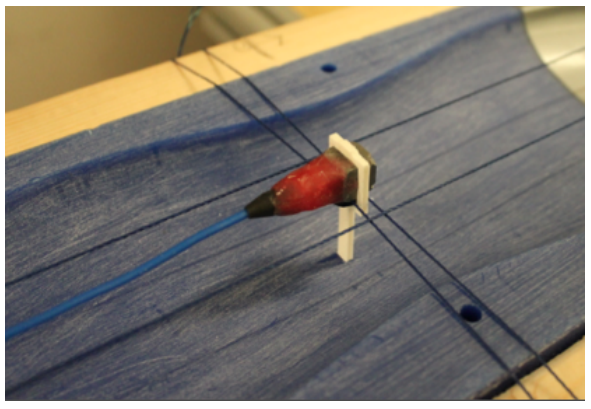

(a)

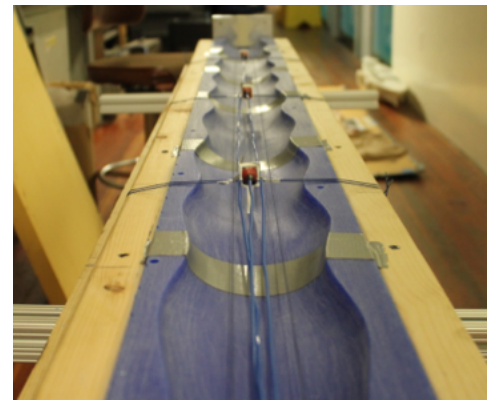

(b)

Figure C-2: (Left) Accelerometer held in place via strings and a thin piece of plastic. (Right) Three accelerometers and two Kevlar strings inside of the mold just before casting.

casting. They were placed evenly along the span with one in the middle. They were held in place via strings strewn across the diameter of the mold and a thin piece of plastic in the direction of the other diameter (Figure C-2a). Figure C-2b shows all the items (three accelerometers and two Kevlar strings) inside of the mold before the rubber was poured in.

\section{C.2.2 Mounting Scheme}

After experimentation with various boundary conditions, it was determined that "clamped-clamped" was the best option. Because of the weight of the model, the model tended to sag along the span. The strings alone were insufficient to keep the body level along its span, so fixed boundary conditions helped reduce this effect. The clamps were used to support a majority of the weight and the strings provided the additional tension needed to pull the body to a horizontal position.

A schematic of the setup is shown in Figure C-3a, and Figure C-3b shows the entire system mounted in the Towing Tank.

\section{C.2.3 Modeling}

The expected natural frequencies of the structure can be calculated using beam theory with the appropriate boundary conditions and assuming the body is in a vacuum. 


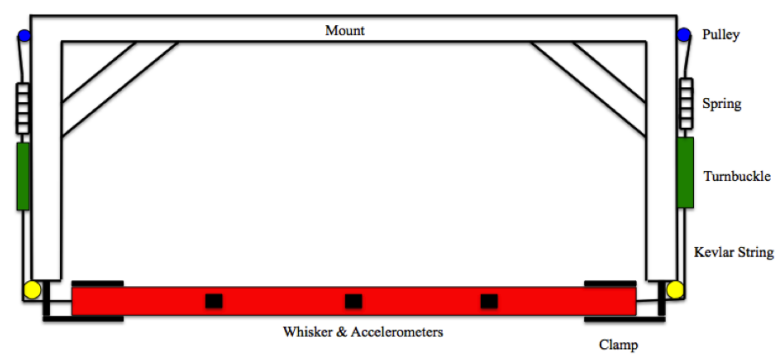

(a)

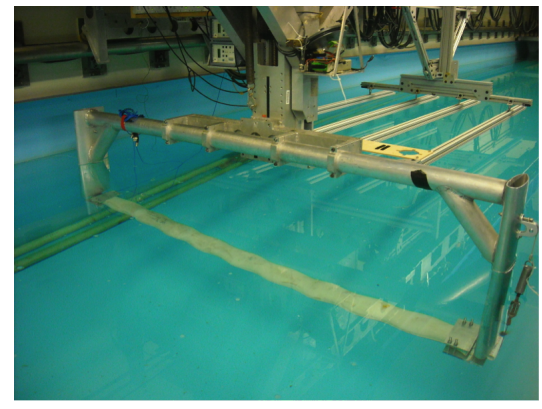

(b)

Figure C-3: (a) Schematic of the setup. The whisker is held with clamps on the end. Turnbuckles are used to apply an initial tension to the Kevlar strings. The spring and pulley system on both ends allows for additional stretching as vibrations occur along the body. (b) Picture of the assembled system mounted in the Towing Tank.

The structure can be modeled as a beam and as a string. The natural frequency is calculated for both cases and combined to determine the overall natural frequency, as shown in equations C.1. This system is seen to be tension-dominated. The calculation is repeated for the first three modes of vibration $(n=1,2,3)$. A pluck test was done on the structure while in air and showed the first mode to vibrate at $f_{n \text {,air }}=2.54 \mathrm{~Hz}$.

$$
\begin{gathered}
f_{\text {beam }}=n^{2} \frac{\pi}{2} \sqrt{\frac{E I}{m L^{4}}}=[0.2,0.9,2.0] \mathrm{Hz} \\
f_{\text {string }}=n \frac{1}{2} \sqrt{\frac{T}{m_{s} L^{2}}}=[2.7,5.5,8.2] \mathrm{Hz} \\
f_{\text {total }}=\sqrt{f_{\text {string }}^{2}+f_{\text {beam }}^{2}}=[2.73,5.53,8.43] \mathrm{Hz}
\end{gathered}
$$

The expected natural frequency in water can be calculated using the expected value in air and incorporating the appropriate added mass and mass ratio values, as in equation C.2. In this case, the body is approximated as a uniform ellipse with a 2:1 diameter ratio, giving it an added mass coefficient $C_{m}$ of 4 . The mass ratio $m^{*}=1.38$ was determined from the Smooth-On PMC-724 material's data sheet value for specific gravity. A crossflow direction pluck test on the model in water showed an oscillation frequency of $f_{n, w a t e r}=1.416 \mathrm{~Hz}$, which matches the expected value very well. 


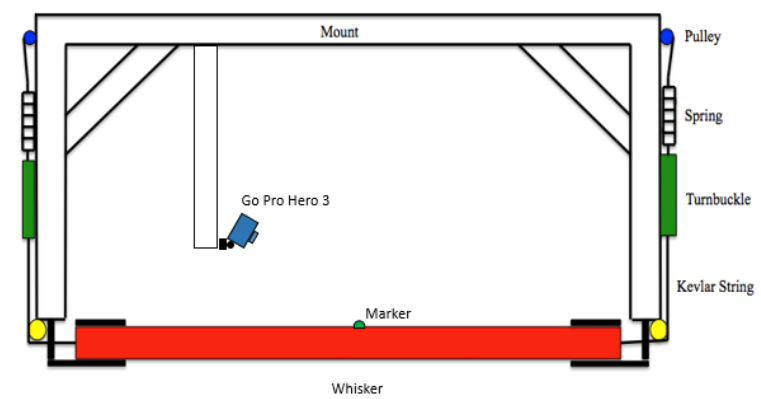

Figure C-4: Schematic of the setup, which now includes a GoPro camera to capture motion of markers on top of the whisker at center span.

$$
f_{\text {water }}=\sqrt{\frac{f_{\text {air }}^{2} m^{*}}{m^{*}+C_{m}}}=[1.4,2.8,4.3] \mathrm{Hz}
$$

\section{C.2.4 Motion Tracking}

Matthew Hildner developed a second means of measuring vibrational response. Optical measurements of the whisker's motion were collected through use of an underwater camera and markers on the whisker surface. This method allows for quantification of motion in all three degrees of freedom.

A schematic of the modified setup is shown in Figure C-4. A GoPro Hero 3 is mounted $\sim 12 \mathrm{in}$ above the whisker to minimize flow disturbance seen by the whisker. It is oriented with a $30^{\circ}$ angle to the markers so as to capture all three degrees of motion simultaneously.

\section{Camera Calibration}

Calibration of the camera from pixels to dimensional length was carried out. Because of the orientation angle of the camera, the distance in the image of the crossflow direction (depicted with the marked pole in Figure C-5) changes exponentially.

\section{Video Processing}

An object tracking algorithm was developed in MATLAB based on a few existing toolboxes. First, the GoPro Cineform software was used to adjust the color of the 


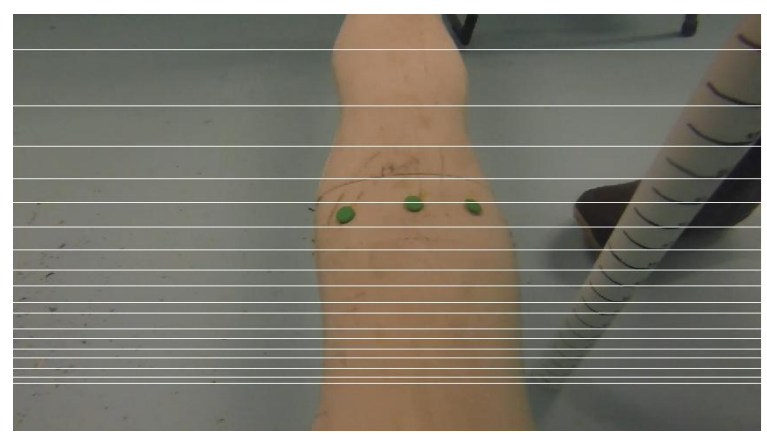

Figure C-5: A calibration image. In this case a pole marked with 1 in increments is used to determine the pixel to crossflow distance conversion.

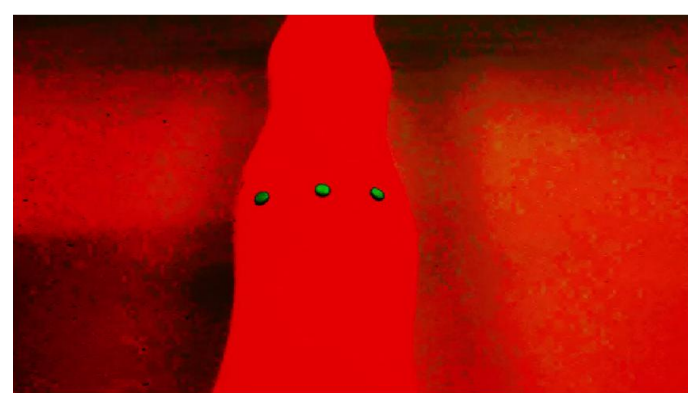

(a)

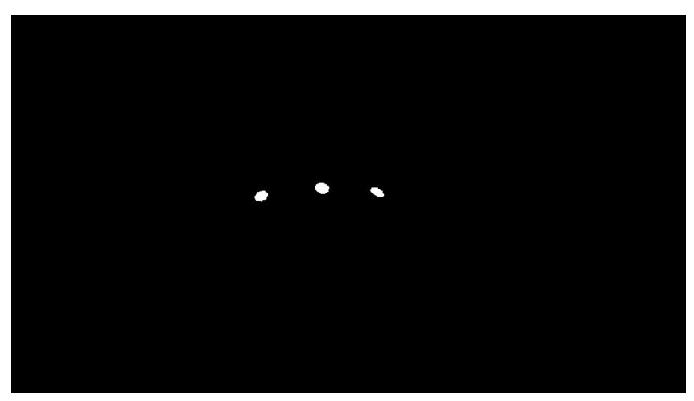

(b)

Figure C-6: A few of the color adjustments done to better highlight the markers.

images. This helped isolate the green thumb tack markers from the white whisker body and blue tank. All objects besides the markers were converted to a red (Figure C-6a). Then a series of transformations were done in MATLAB, leaving a clear contrast of white markers against a black background (Figure C-6b).

Next, MATLAB's Blob Analyzer was used to identify the groupings of white pixels and track their location over time. A Kalman filter predicts the location of the markers for the next frame, which helps reduce incorrect tracking.

\section{C.3 Results}

\section{C.3.1 Accelerometer}

Data from the middle accelerometer in the crossflow direction was collected. This signal was integrated two times, detrended, and filtered with a low-pass Butterworth 

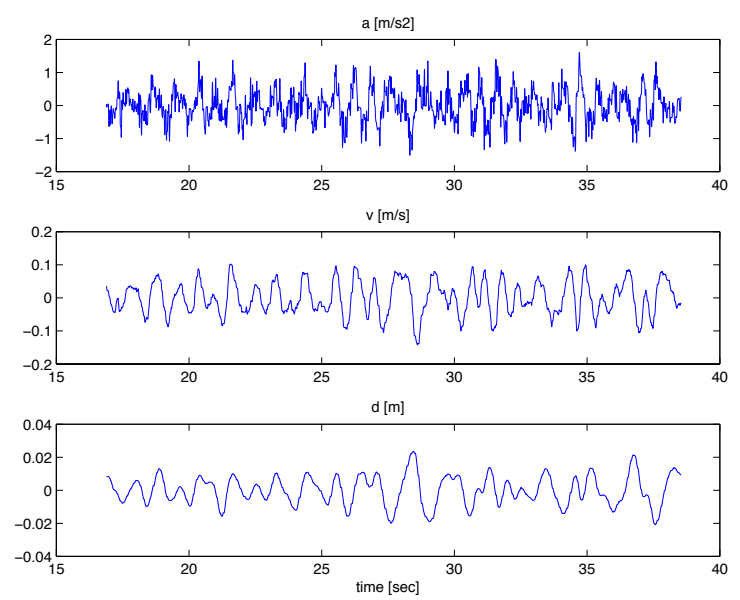

Figure C-7: From top to bottom: crossflow acceleration, velocity, and displacement of the whisker as measured from the middle accelerometer in the $U_{n}^{*}=4$ case.

filter to estimate the displacement. An example plot of the progression from acceleration to velocity to displacement is shown in Figure C-7.

\section{C.3.2 Motion Tracking of Model}

The markers were tracked over the course of various towing tank runs, their positions were converted to dimensional lengths, and their amplitudes were calculated in terms of root-mean-square $(r m s)$. The results are compiled in Figure C-8a along with the results from the accelerometer. The dominant frequencies were measured using both techniques by selecting the peak response in the FFT of the signal and nondimensionalizing by the crossflow natural frequency in air (Figure C-8b).

The two measurement techniques line up reasonably well with each other. Both show a higher peak value than those measured in the rigid whisker experiments. A high response is seen to start to develop at high velocities. This is, however, not believed to necessarily indicate galloping because the model was observed to undergo 


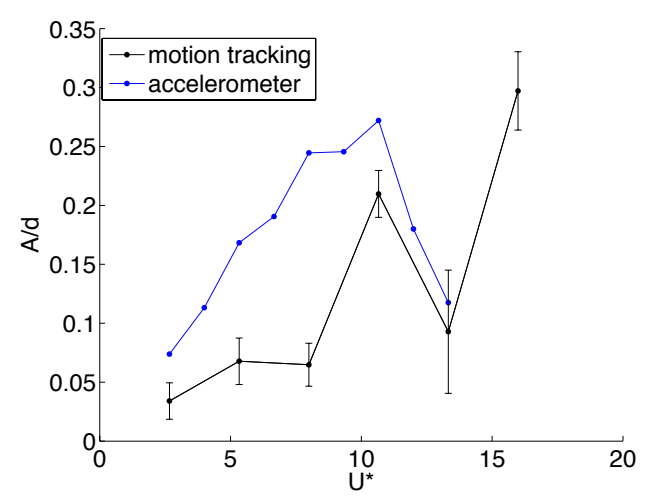

(a)

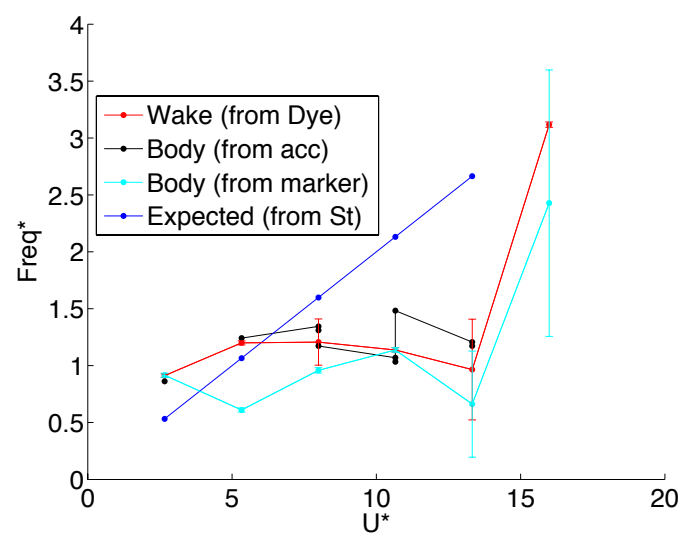

(b)

Figure C-8: The nondimensional crossflow (a) amplitude and (b) frequency measured both by the accelerometers and by the motion tracking software.
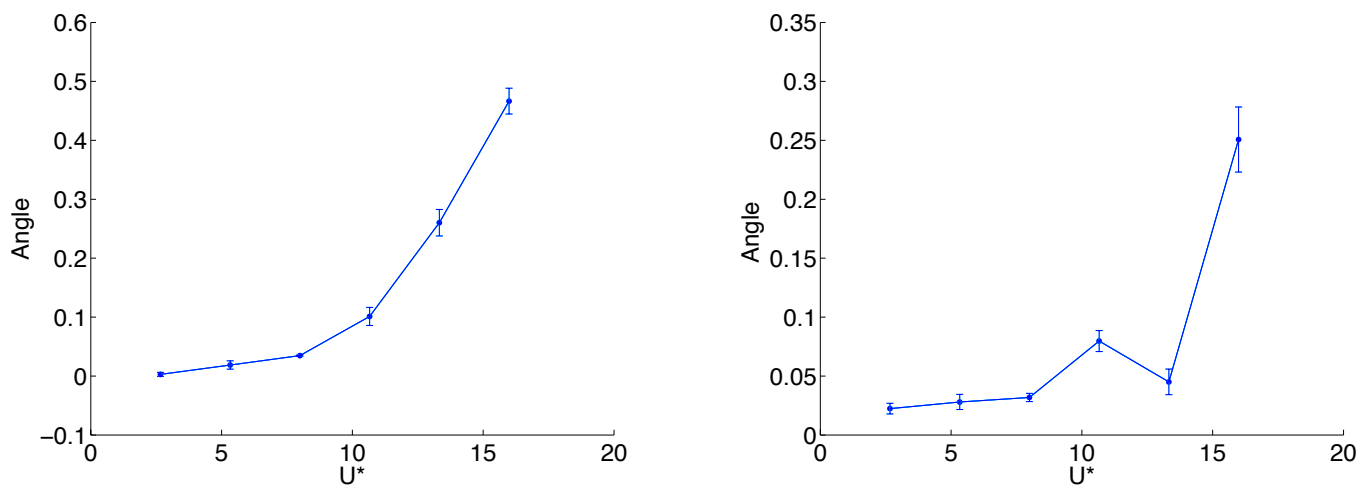

Figure C-9: (a) Mean and (b) rms rotation of the model across different towing velocities. Both increase dramatically at high speeds.

significant mean rotation along the spanwise axis at high speeds. The model could no longer be considered as being tested at $\alpha=0^{\circ}$.

The rotation was quantified through the motion tracking software. Both mean rotation and rms rotation are shown in Figure C-9. As towing speed increases, the mean rotation increases. The rms rotation is non-zero in all cases, and reaches a peak at $U=0.8 \mathrm{~m} / \mathrm{s}$. 


\section{C.4 Discussion and Future Work}

Both in-line and crossflow vibrations reach higher values in this flexible whisker setup than in the rigid whisker experiments. Also, a significant torsional excitation was measured, especially at certain speeds. The excitation of this mode may be driven by the elliptical cross section of the geometry [137].

It is possible that torsional vibration is responsible for inducing the additional vibrations seen in the crossflow and in-line directions. To investigate this, we are modifying the freely vibrating rigid-whisker system to include a torsional degree of freedom. 


\section{Bibliography}

[1] A.S. Ahl. The role of vibrissae in behavior: a status review. Veterinary research communications, 10(1):245-268, 1986.

[2] A. Ahmed and B. Bays-Muchmore. Transverse flow over a wavy cylinder. Physics of Fluids A: Fluid Dynamics (1989-1993), 4(9):1959-1967, 1992.

[3] G.R.S. Assi, P.W. Bearman, B.S. Carmo, J.R. Meneghini, S.J. Sherwin, and R.H.J. Willden. The role of wake stiffness on the wake-induced vibration of the downstream cylinder of a tandem pair. Journal of Fluid Mechanics, 718:210$245,2013$.

[4] G.R.S. Assi, P.W. Bearman, and J.R. Meneghini. On the wake-induced vibration of tandem circular cylinders: the vortex interaction excitation mechanism. Journal of Fluid Mechanics, 661:365-401, 2010.

[5] G.R.S. Assi, J.R. Meneghini, J.A.P. Aranha, P.W. Bearman, and E. Casaprima. Experimental investigation of flow-induced vibration interference between two circular cylinders. Journal of Fluids and Structures, 22(6):819-827, 2006.

[6] C. Barbier, J.A.C. Humphrey, J. Paulus, and M. Appleby. Design, fabrication and testing of a bioinspired hybrid hair-like fluid motion sensor array. In ASME 2007 International Mechanical Engineering Congress and Exposition, pages 1319-1324, 2007.

[7] D.N. Beal, F.S. Hover, M.S. Triantafyllou, J.C. Liao, and G.V. Lauder. Passive propulsion in vortex wakes. Journal of Fluid Mechanics, 549:385-402, 2006.

[8] P.W. Bearman. Vortex shedding from oscillating bluff bodies. Annual Review of Fluid Mechanics, 16(1):195-222, 1984.

[9] O. Bebek and M.C. Cavusoglu. Whisker-like position sensor for measuring physiological motion. IEEE/ASME Transactions on Mechatronics, 13(5):538547, 2008.

[10] H. Beem, M. Hildner, and M. Triantafyllou. Calibration and validation of a harbor seal whisker-inspired flow sensor. Smart Materials and Structures, 22(1):014012, 2013.

[11] R.D. Blevins. Flow-induced vibrations. Van Nostrand Reinhold, 1990. 
[12] W.D. Bowen, D. Tully, D.J. Boness, B.M. Bulheier, and G.J. Marshall. Preydependent foraging tactics and prey profitability in a marine mammal. Marine Ecology Progress Series, 244:235-245, 2002.

[13] D.M. Bushnell and K.J. Moore. Drag reduction in nature. Annual Review of Fluid Mechanics, 23(1):65-79, 1991.

[14] K.C. Catania. Tactile sensing in specialized predators-from behavior to the brain. Current opinion in neurobiology, 22(2):251-258, 2012.

[15] K.C. Catania, D.B. Leitch, and D. Gauthier. Function of the appendages in tentacled snakes (Erpeton tentaculatus). Journal of Experimental Biology, 213(3):359-367, 2010.

[16] N. Czech. Functional Morphology and Postnatal Transformation of Vibrissal Crypts in Toothed Whales (Odontoceti). PhD thesis, Ruhr-Universität Bochum, 2007.

[17] J.M. Dahl, F.S. Hover, M.S. Triantafyllou, and O.H. Oakley. Dual resonance in vortex-induced vibrations at subcritical and supercritical reynolds numbers. Journal of Fluid Mechanics, 643(1):395-424, 2010.

[18] R.W. Davis, L.A. Fuiman, T.M. Williams, S.O. Collier, W.P. Hagey, S.B. Kanatous, S. Kohin, and M. Horning. Hunting behavior of a marine mammal beneath the antarctic fast ice. Science, 283(5404):993-996, 1999.

[19] G. Dehnhardt. Preliminary results from psychophysical studies on the tactile sensitivity in marine mammals. In Sensory abilities of cetaceans, pages 435-446. Springer, 1990.

[20] G. Dehnhardt. Tactile size discrimination by a california sea lion (Zalophus californianus) using its mystacial vibrissae. Journal of Comparative Physiology A, 175(6):791-800, 1994.

[21] G. Dehnhardt, W. Hanke, S. Wieskotten, Y. Krüger, and L. Miersch. Hydrodynamic perception in seals and sea lions. In Flow Sensing in Air and Water, pages 147-167. Springer, 2014.

[22] G. Dehnhardt, H. Hyvärinen, A. Palviainen, and G. Klauer. Structure and innervation of the vibrissal follicle-sinus complex in the australian water rat, Hydromys chrysogaster. Journal of Comparative Neurology, 411(4):550-562, 1999.

[23] G. Dehnhardt and A. Kaminski. Sensitivity of the mystacial vibrissae of harbour seals (Phoca vitulina) for size differences of actively touched objects. Journal of Experimental Biology, 198(11):2317-23, 1995.

[24] G. Dehnhardt, B. Mauck, and H. Bleckmann. Seal whiskers detect water movements. Nature, 394(6690):235-236, 071998. 
[25] G. Dehnhardt, B. Mauck, W. Hanke, and H. Bleckmann. Hydrodynamic trailfollowing in harbor seals (Phoca vitulina). Science, 293(5527):102-104, 2001.

[26] G. Dehnhardt, B. Mauck, and H. Hyvärinen. The functional significance of the vibrissal system of marine mammals. In The Merkel Cell, pages 127-135. Springer, 2003.

[27] G. Dehnhardt, M. Sinder, and N. Sachser. Tactual discrimination of size by means of mystacial vibrissae in harbour seals: in air versus underwater. Zeitschrift für Säugetierkunde, 62:40-43, 1997.

[28] M.A. Donelan and J. Motycka. Miniature drag sphere velocity probe. Review of Scientific Instruments, 49(3):298-304, 1978.

[29] E.G. Drucker and G.V. Lauder. Experimental hydrodynamics of fish locomotion: functional insights from wake visualization. Integrative and Comparative Biology, 42(2):243-257, 2002.

[30] R.W. Dykes. What the seal's vibrissae tells the seal's brain. In Proceedings of the ninth annual Conference on Biological, Sonar and Diving Mammals held at Stanford Research Institute, Biological Sonar Laboratory, Menlo Park, California, pages 123-136, 1970.

[31] R.W. Dykes. Afferent fibers from mystacial vibrissae of cats and seals. Journal of Neurophysiology, 38(3):650-662, 1975.

[32] W.C. Eberhardt, Y.A. Shakhsheer, B.H. Calhoun, J.R. Paulus, and M. Appleby. A bio-inspired artificial whisker for fluid motion sensing with increased sensitivity and reliability. In 2011 IEEE Sensors, pages 982-985, 2011.

[33] R. Elsner, D. Wartzok, N.B. Sonafrank, and B.P. Kelly. Behavioral and physiological reactions of arctic seals during under-ice pilotage. Canadian journal of zoology, 67(10):2506-2513, 1989.

[34] N. Erdsack, G. Dehnhardt, and W. Hanke. Thermoregulation of the vibrissal system in harbor seals (Phoca vitulina) and cape fur seals (Arctocephalus pusillus pusillus). Journal of Experimental Marine Biology and Ecology, 452:111118, 2014.

[35] M.J. Every, R. King, and D.S. Weaver. Vortex-excited vibrations of cylinders and cables and their suppression. Ocean Engineering, 9(2):135-157, 1982.

[36] C.C. Feng. The measurement of vortex induced effects in flow past stationary and oscillating circular and d-section cylinders. Master's thesis, University of British Columbia, 1968.

[37] F. Flemming and C.H.K. Williamson. Vortex-induced vibrations of a pivoted cylinder. Journal of Fluid Mechanics, 522:215-252, 2005. 
[38] A.S. French. Transduction mechanisms of mechanosensilla. Annual Review of Entomology, 33(1):39-58, 1988.

[39] Y. Gao, Z. Sun, D.S. Tan, D. Yu, and S.K. Tan. Wake flow behaviour behind a smaller cylinder oscillating in the wake of an upstream stationary cylinder. Fluid Dynamics Research, 46(2):025505, 2014.

[40] Y. Gao, D. Yu, S. Tan, X. Wang, and Z. Hao. Experimental study on the near wake behind two side-by-side cylinders of unequal diameters. Fluid dynamics research, 42(5):055509, 2010.

[41] Y.Y. Gao, X. Wang, D.S. Tan, and T.S. Keat. Particle image velocimetry technique measurements of the near wake behind a cylinder-pair of unequal diameters. Fluid Dynamics Research, 45(4):045504, 2013.

[42] J.C. Gaspard III, G.B. Bauer, R.L. Reep, K. Dziuk, L. Read, and D.A. Mann. Detection of hydrodynamic stimuli by the florida manatee (Trichechus manatus latirostris). Journal of Comparative Physiology A, 199(6):441-450, 2013.

[43] H.F. Gaydos. Sensitivity in the judgment of size by finger-span. The American journal of psychology, pages 557-562, 1958.

[44] C.D. Gerber. Design and vibration testing of a flexible seal whisker model. B.S. Thesis, Massachusetts Institute of Technology, 2013.

[45] C.C. Ginter, T.J. DeWitt, F.E. Fish, and C.D. Marshall. Fused traditional and geometric morphometrics demonstrate pinniped whisker diversity. PLoS ONE, 7(4):e34481, 042012.

[46] C.C. Ginter, F.E. Fish, and C.D. Marshall. Morphological analysis of the bumpy profile of phocid vibrissae. Marine Mammal Science, 26(3):733-743, 2010.

[47] N. Gläser, B. Mauck, F.I. Kandil, M. Lappe, G. Dehnhardt, and F.D. Hanke. Harbor seals (Phoca vitulina) can perceive optic flow under water. PLoS ONE, 9(7):e103555, 072014.

[48] N. Gläser, S. Wieskotten, C. Otter, G. Dehnhardt, and W. Hanke. Hydrodynamic trail following in a california sea lion (Zalophus californianus). Journal of Comparative Physiology A: Neuroethology, Sensory, Neural, and Behavioral Physiology, 197:141-151, 2011. 10.1007/s00359-010-0594-5.

[49] R. Gopalkrishnan. Vortex-induced forces on oscillating bluff cylinders. PhD thesis, Massachusetts Institute of Technology/Woods Hole Oceanographic Institution Joint Program, 1993.

[50] R.N. Govardhan and C.H.K. Williamson. Defining the 'modified griffin plot' in vortex-induced vibration: revealing the effect of reynolds number using controlled damping. Journal of Fluid Mechanics, 561:147-180, 2006. 
[51] D.K. Greaves, M.O. Hammill, J.D. Eddington, D. Pettipas, and J.F. Schreer. Growth rate and shedding of vibrissae in the gray seal, Halichoerus Grypus: A cautionary note for stable isotope diet analysis. Marine Mammal Science, 20(2):296-304, 2004.

[52] S. Große and W. Schröder. Deflection-based flow field sensors-examples and requirements. In Frontiers in Sensing, pages 393-403. Springer, 2012.

[53] F.D. Hanke, W. Hanke, C. Scholtyssek, and G. Dehnhardt. Basic mechanisms in pinniped vision. Experimental brain research, 199(3-4):299-311, 2009.

[54] W. Hanke and H. Bleckmann. The hydrodynamic trails of Lepomis gibbosus (centrarchidae), Colomesus psittacus (tetraodontidae) and Thysochromis ansorgii (cichlidae) investigated with scanning particle image velocimetry. Journal of Experimental Biology, 207(9):1585-1596, 2004.

[55] W. Hanke, C. Brucker, and H. Bleckmann. The ageing of the low-frequency water disturbances caused by swimming goldfish and its possible relevance to prey detection. Journal of Experimental Biology, 203(7):1193-1200, 2000.

[56] W. Hanke, S. Wieskotten, C. Marshall, and G. Dehnhardt. Hydrodynamic perception in true seals (phocidae) and eared seals (otariidae). Journal of Comparative Physiology A, pages 1-20, 2012.

[57] W. Hanke, S. Wieskotten, B. Niesterok, L. Miersch, M. Witte, M. Brede, A. Leder, and G. Dehnhardt. Nature-Inspired Fluid Mechanics: Results of the DFG Priority Programme 1207, chapter "Hydrodynamic Perception in Pinnipeds", pages 255-270. Springer, 2012.

[58] W. Hanke, M. Witte, L. Miersch, M. Brede, J. Oeffner, M. Michael, F. Hanke, A. Leder, and G. Dehnhardt. Harbor seal vibrissa morphology suppresses vortex-induced vibrations. Journal of Experimental Biology, 213(15):2665-2672, 2010.

[59] H. Hans, J.M. Miao, and M.S. Triantafyllou. Mechanical characteristics of harbor seal (Phoca vitulina) vibrissae under different circumstances and their implications on its sensing methodology. Bioinspiration $\&$ biomimetics, 9(3):036013, 2014.

[60] M. Harrison. British Mammals, volume The New Naturalist. Collins, 1952.

[61] M.J. Hartmann, N.J. Johnson, R.B. Towal, and C. Assad. Mechanical characteristics of rat vibrissae: Resonant frequencies and damping in isolated whiskers and in the awake behaving animal. Journal of Neuroscience, 23(16):6510-6519, 2003.

[62] A.C. Hirons, D.M. Schell, and D.J. St. Aubin. Growth rates of vibrissae of harbor seals (Phoca vitulina) and steller sea lions (Eumetopias jubatus). Canadian Journal of Zoology, 79(6):1053-1061, 2001. 
[63] E.S. Hobson. Visual orientation and feeding in seals and sea lions. Nature, 210(5033):326-327, 041966.

[64] F.S. Hover, A.H. Techet, and M.S. Triantafyllou. Forces on oscillating uniform and tapered cylinders in crossflow. Journal of Fluid Mechanics, 363:97-114, 1998.

[65] F.S. Hover and M.S. Triantafyllou. Galloping response of a cylinder with upstream wake interference. Journal of fluids and structures, 15(3):503-512, 2001.

[66] http://www.exponent.com/files/Uploads/Images/Energy/oil\%20platform.jpg.

[67] http://www.marine-science center.de.

[68] H. Hyvärinen. On the histology and histochemistry of the snout and vibrissae of the common shrew (Sorex araneus 1.). Zeitschrift für Zellforschung und Mikroskopische Anatomie, 124(4):445-453, 1972.

[69] H. Hyvärinen. Diving in darkness: whiskers as sense organs of the ringed seal (Phoca hispida saimensis). Journal of Zoology, 218(4):663-678, 1989.

[70] H. Hyvärinen. Structure and function of the vibrissae of the ringed seal (Phoca hispida L.). De Spil Publishers, Woerden, The Netherlands, 1995.

[71] H. Hyvärinen and H. Katajisto. Functional structure of the vibrissae of the ringed seal (Phoca hispida schr.). Acta Zoologica Fennica, 171:17-30, 1984.

[72] H. James and R.W. Dykes. Some experiments on navigation in the harbour seal, Phoca vitulina. In Animal Migration, Navigation, and Homing, pages 395-404. Springer, 1978.

[73] A.J. Kalmijn. Detection of weak electric fields. In Sensory biology of aquatic animals, pages 151-186. Springer, 1988.

[74] R.A. Kastelein and M.A. Van Gaalen. The sensitivity of the vibrissae of a pacific walrus (Odobenus rosmarus divergem) part 1. Aquatic Mammals, 14:123-133, 1988.

[75] J. Kim and H. Choi. Distributed forcing of flow over a circular cylinder. Physics of Fluids, 17(3):033103, 2005.

[76] M.H. Kim, J.W. Lee, H.S. Yoon, and M.Y. Ha. Numerical study on the flow past a twisted elliptic cylinder with subcritical reynolds number. In $A S M E-J S M E$ KSME 2011 Joint Fluids Engineering Conference, pages 3885-3893, 2011.

[77] Gerald L Kooyman. An analysis of some behavioral and physiological characteristics related to diving in the Weddell seal. Wiley Online Library, 1968. 
[78] S. Kowalewsky, M. Dambach, B. Mauck, and G. Dehnhardt. High olfactory sensitivity for dimethyl sulphide in harbour seals. Biology Letters, 2(1):106$109,2006$.

[79] T.F. Ladygina, V.V. Popov, and A.Y. Supin. Topical organization of somatic projections in the fur seal cerebral cortex. Neurophysiology, 17(3):246-252, 1985.

[80] K. Lam and Y.F. Lin. Effects of wavelength and amplitude of a wavy cylinder in cross-flow at low reynolds numbers. Journal of Fluid Mechanics, 620:195-220, 2009.

[81] K. Lam, F.H. Wang, J.Y. Li, and R.M.C. So. Experimental investigation of the mean and fluctuating forces of wavy (varicose) cylinders in a cross-flow. Journal of Fluids and Structures, 19(3):321 - 334, 2004.

[82] K.M. Lam and A.P. To. Interference effect of an upstream larger cylinder on the lock-in vibration of a flexibly mounted circular cylinder. Journal of fluids and structures, 17(8):1059-1078, 2003.

[83] S.J. Lee and A.T. Nguyen. Experimental investigation on wake behind a wavy cylinder having sinusoidal cross-sectional area variation. Fluid Dynamics Research, 39(4):292 - 304, 2007.

[84] D.H. Levenson and R.J. Schusterman. Dark adaptation and visual sensitivity in shallow and deep-diving pinnipeds. Marine Mammal Science, 15(4):1303-1313, 1999.

[85] J.C. Liao, D.N. Beal, G.V. Lauder, and M.S. Triantafyllou. Fish exploiting vortices decrease muscle activity. Science, 302(5650):1566-1569, 2003.

[86] J.K. Ling. The skin and hair of the southern elephant seal, Mirounga leonina (linn.) i. the facial vibrissae. Australian Journal of Zoology, 14(5):855-866, 1966.

[87] J.K. Ling. Vibrissae of marine mammals. Functional anatomy of marine mammals, 3:387-415, 1977.

[88] W.R. Lowell and W.F. Flanigan. Marine mammal chemoreception. Mammal review, 10(1):53-59, 1980.

[89] S. Marriott, E. Cowan, J. Cohen, and R.M. Hallock. Somatosensation, echolocation, and underwater sniffing: Adaptations allow mammals without traditional olfactory capabilities to forage for food underwater. Zoological Science, 30:6975, 2013.

[90] B. Mauck, U. Eysel, and G. Dehnhardt. Selective heating of vibrissal follicles in seals (Phoca vitulina) and dolphins (Sotalia fluviatilis guianensis). Journal of Experimental Biology, 203(14):2125-2131, 2000. 
[91] L. Miersch, W. Hanke, S. Wieskotten, F.D. Hanke, J. Oeffner, A. Leder, M. Brede, M. Witte, and G. Dehnhardt. Flow sensing by pinniped whiskers. Philosophical Transactions of the Royal Society B: Biological Sciences, 366(1581):3077-3084, 2011.

[92] F.H.J. Mills and D. Renouf. Determination of the vibration sensitivity of harbour seal Phoca vitulina (1.) vibrissae. Journal of Experimental Marine Biology and Ecology, 100(1-3):3-9, 1986.

[93] T.L. Morse and C.H.K. Williamson. Employing controlled vibrations to predict fluid forces on a cylinder undergoing vortex-induced vibrations. Journal of Fluids and Structures, 22(6-7):877-884, 2006.

[94] T.L. Morse and C.H.K. Williamson. Prediction of vortex-induced vibration response by employing controlled motion. Journal of Fluid Mechanics, 634:539, 2009.

[95] C.T. Murphy, W.C. Eberhardt, B.H. Calhoun, K.A. Mann, and D.A. Mann. Effect of angle on flow-induced vibrations of pinniped vibrissae. PloS one, 8(7):e69872, 2013.

[96] Y. Naito, D. Costa, T. Adachi, P.W. Robinson, M. Fowler, and A. Takahashi. Unravelling the mysteries of a mesopelagic diet: a large apex predator specializes on small prey. Functional Ecology, 27(3):710-717, 2013.

[97] B. Niesterok and W. Hanke. Hydrodynamic patterns from fast-starts in teleost fish and their possible relevance to predator-prey interactions. Journal of Comparative Physiology A, 199(2):139-149, 2013.

[98] G.W. Oliver. Navigation in mazes by a grey seal, halichoerus grypus (fabricius). Behaviour, 67:97-114, 1978.

[99] J.C. Owen, P.W. Bearman, and A.A. Szewczyk. Passive control of viv with drag reduction. Journal of Fluids and Structures, 15(3-4):597-605, 2001.

[100] J.C. Owen, A.A. Szewczyk, and P.W. Bearman. Suppression of karman vortex shedding. Phys. Fluids, 12(9):S9, 2000.

[101] Y. Ozaki, T. Ohyama, T. Yasuda, and I. Shimoyama. An air flow sensor modeled on wind receptor hairs of insects. In The Thirteenth Annual International Conference on Micro Electro Mechanical Systems 2000, pages 531-536, 2000.

[102] G.V. Papaioannou, D.K.P. Yue, M.S. Triantafyllou, and G.E. Karniadakis. Three-dimensionality effects in flow around two tandem cylinders. Journal of Fluid Mechanics, 558:387-413, 2006.

[103] K. Pohlmann, J. Atema, and T. Breithaupt. The importance of the lateral line in nocturnal predation of piscivorous catfish. Journal of Experimental Biology, 207(17):2971-2978, 2004. 
[104] K. Pohlmann, F.W. Grasso, and T. Breithaupt. Tracking wakes: the nocturnal predatory strategy of piscivorous catfish. Proceedings of the National Academy of Sciences, 98(13):7371-7374, 2001.

[105] T.C. Poulter. Sonar signals of the sea lion. Science, 139(3556):753-755, 1963.

[106] R.L. Reep, J.C. Gaspard, D. Sarko, F.L. Rice, D.A. Mann, and G.B. Bauer. Manatee vibrissae: evidence for a "lateral line" function. Annals of the New York Academy of Sciences, 1225(1):101-109, 2011.

[107] D. Renouf. Preliminary measurements of the sensitivity of the vibrissae of harbour seals (Phoca vitulina) to low frequency vibrations. Journal of Zoology, 188(4):443-450, 1979.

[108] D. Renouf. Fishing in captive harbour seals (Phoca Vitulina Concolor): a possible role for vibrissae. Netherlands journal of zoology, 30(3):504-509, 1980.

[109] D. Renouf. Sensory function in the harbor seal. Scientific American, 260(4):9095, 1989.

[110] D. Renouf. Sensory reception and processing in phocidae and otariidae. In The behaviour of pinnipeds, pages 345-394. Springer, 1991.

[111] D. Renouf and L. Gaborko. Speed sensing in a harbour seal. Journal of the Marine Biological Association of the United Kingdom, 62(01):227-228, 1982.

[112] W.J. Richardson. Marine Mammal Sounds. San Diego Academic Press, 1995.

[113] A. Roshko. On the development of turbulent wakes from vortex streets. Technical Report 1191, National Advisory Committee for Aeronautics, 1954.

[114] T. Sarpkaya. A critical review of the intrinsic nature of vortex-induced vibrations. Journal of Fluids and Structures, 19(4):389-447, 2004.

[115] A.T. Sayers and A. Saban. Flow over two cylinders of different diameters: The lock-in effect. Journal of Wind Engineering and Industrial Aerodynamics, 51(1):43-54, 1994.

[116] V.B. Scheffer. Seals, sea lions, and walruses: a review of the Pinnipedia. Stanford University Press, 1958.

[117] V.B. Scheffer and J.W. Slipp. The harbor seal in washington state. American Midland Naturalist, pages 373-416, 1944.

[118] N. Schulte-Pelkum, S. Wieskotten, W. Hanke, G. Dehnhardt, and B. Mauck. Tracking of biogenic hydrodynamic trails in harbour seals (Phoca vitulina). Journal of Experimental Biology, 210(5):781-787, 2007. 
[119] R.J. Schusterman, D. Kastak, D.H. Levenson, C.J. Reichmuth, and B.L. Southall. Why pinnipeds don't echolocate. Journal of the Acoustical Society of America, 107(4):2256-2264, 2000.

[120] K. Schwenk. Why snakes have forked tongues. Science, 263(5153):1573-1577, 1994.

[121] L.F. Shatz and T. De Groot. The frequency response of the vibrissae of harp seal, Pagophilus groenlandicus, to sound in air and water. PloS one, 8(1):e54876, 2013.

[122] J.H. Solomon and M.J. Hartmann. Biomechanics: robotic whiskers used to sense features. Nature, 443(7111):525-525, 2006.

[123] G.R. Spedding. The evolution of initially turbulent bluff-body wakes at high internal froude number. Journal of Fluid Mechanics, 337:283-301, 1997.

[124] T. Staubli. Calculation of the vibration of an elastically mounted cylinder using experimental data from forced oscillation. J. Fluids Eng, 105(225-229):C367, 1983.

[125] J. Sticken and G. Dehnhardt. Salinity discrimination in harbour seals: a sensory basis for spatial orientation in the marine environment? Naturwissenschaften, 87(11):499-502, 2000.

[126] D. Sumner, J.L. Heseltine, and O.J.P. Dansereau. Wake structure of a finite circular cylinder of small aspect ratio. Experiments in Fluids, 37(5):720-730, 2004.

[127] J. Tao and X.B. Yu. Hair flow sensors: from bio-inspiration to bio-mimicking-a review. Smart Materials and Structures, 21(11):113001, 2012.

[128] A. Thesen, J.B. Steen, and K.B. Doving. Behaviour of dogs during olfactory tracking. Journal of Experimental Biology, 180(1):247-251, 1993.

[129] G.S. Triantafyllou, M.S. Triantafyllou, and M.A. Grosenbaugh. Optimal thrust development in oscillating foils with application to fish propulsion. Journal of Fluids and Structures, 7(2):205-224, 1993.

[130] P. Valdivia y Alvarado, V. Subramaniam, and M. Triantafyllou. Design of a bio-inspired whisker sensor for underwater applications. In Proceedings of IEEE Sensors 2012, Taipei, Taiwan, 2012.

[131] J.K. Vandiver and T.Y. Chung. Hydrodynamic damping on flexible cylinders in sheared flow. Journal of Waterway, Port, Coastal, and Ocean Engineering, 115(2):154-171, 1989.

[132] D. Vogel and H. Bleckmann. Behavioral discrimination of water motions caused by moving objects. Journal of Comparative Physiology A, 186(12):1107-1117, 2001. 
[133] D.E. Walshe and L.R. Wootton. Preventing wind-induced oscillations of structures of circular section. In ICE Proceedings, volume 47, pages 1-24. Thomas Telford, 1970.

[134] W.A. Watkins and D. Wartzok. Sensory biophysics of marine mammals. Marine Mammal Science, 1(3):219-260, 1985.

[135] J.K Webb and R. Shine. To find an ant: trail-following in australian blindsnakes (typhlopidae). Animal Behaviour, 43(6):941-948, 1992.

[136] M. Weiffen, B. Möller, B. Mauck, and G. Dehnhardt. Effect of water turbidity on the visual acuity of harbor seals (Phoca vitulina). Vision research, 46(11):1777$1783,2006$.

[137] G.D. Weymouth. Chaotic rotation of a towed elliptical cylinder. Journal of Fluid Mechanics, 743:385-398, 2014.

[138] S. Wieskotten, G. Dehnhardt, B. Mauck, L. Miersch, and W. Hanke. Hydrodynamic determination of the moving direction of an artificial fin by a harbour seal (Phoca vitulina). Journal of Experimental Biology, 213(13):2194-2200, 2010.

[139] S. Wieskotten, G. Dehnhardt, B. Mauck, L. Miersch, and W. Hanke. The impact of glide phases on the trackability of hydrodynamic trails in harbour seals (Phoca vitulina). Journal of Experimental Biology, 213(21):3734-3740, 2010 .

[140] S. Wieskotten, B. Mauck, L. Miersch, G. Dehnhardt, and W. Hanke. Hydrodynamic discrimination of wakes caused by objects of different size or shape in a harbour seal (Phoca vitulina). Journal of Experimental Biology, 214(11):19221930, 2011.

[141] C.H.K. Williamson. Vortex dynamics in the cylinder wake. Annual review of fluid mechanics, 28(1):477-539, 1996.

[142] C.H.K. Williamson and R. Govardhan. Vortex-induced vibrations. Annual Review of Fluid Mechanics, 36(1):413-455, 2004.

[143] M. Witte, W. Hanke, S. Wieskotten, L. Miersch, M. Brede, G. Dehnhardt, and A. Leder. Nature-Inspired Fluid Mechanics: Results of the DFG Priority Programme 120\%, chapter "On the Wake Flow Dynamics behind Harbor Seal Vibrissae - A Fluid Mechanical Explanation for an Extraordinary Capability", pages 271-289. Springer, 2012.

[144] M.M. Zdravkovich. Review and classification of various aerodynamic and hydrodynamic means for suppressing vortex shedding. Journal of Wind Engineering and Industrial Aerodynamics, 7(2):145 - 189, 1981.

[145] W. Zhang, Daichin, and S.J. Lee. Piv measurements of the near-wake behind a sinusoidal cylinder. Experiments in Fluids, 38:824-832, 2005. 10.1007/s00348005-0981-9. 